ERIKA MARIA FRANCISCHINELLI FERNANDEZ

CONTROLE PREDITIVO COM ENFOQUE EM SUBESPAÇOS 
ERIKA MARIA FRANCISCHINELLI FERNANDEZ

CONTROLE PREDITIVO COM ENFOQUE EM SUBESPAÇOS

Dissertação apresentada à Escola

Politécnica da Universidade de São Paulo para obtenção do título de Mestre em

Engenharia 


\section{CONTROLE PREDITIVO COM ENFOQUE EM SUBESPAÇOS}

Dissertação apresentada à Escola

Politécnica da Universidade de São Paulo para obtenção do título de Mestre em

Engenharia

Área de Concentração: Engenharia Química

Orientador: Prof. Dr. Darci Odloak 
FICHA CATALOGRÁFICA

Fernandez, Erika Maria Francischinelli

Controle preditivo com enfoque em subespaços / E.M.F.

Fernandez. -- São Paulo, 2009.

p.160

Dissertação (Mestrado) - Escola Politécnica da Universidade de São Paulo. Departamento de Engenharia Química.

1. Controle preditivo 2. Controle de processos 3 . Identifica ção de sistemas I. Universidade de São Paulo. Escola Politécnica. Departamento de Engenharia Química II. t. 


\section{AGRADECIMENTOS}

Ao Prof. Dr. Darci Odloak, pela excelente orientação e pela atenção e paciência em nossas inúmeras conversas.

À minha querida tia Rose, que me ajudou muito revisando este trabalho com tanta atenção.

Aos meus pais, Ana e Roberto, por todo o amor e apoio. A toda a minha família, pela amizade e união.

Ao Prof. Dr. Oscar Sotomayor, por todo material disponibilizado.

Ao Prof. Dr. Cláudio Garcia, com quem aprendi muito.

Aos professores e colegas do LSCP, pelo convívio durante esses dois anos. Em especial, ao Prof. Dr. Galo Le Roux, pelas constantes dúvidas que me tirou.

À OP2B e à CHEMTECH pelo incentivo para a realização deste Mestrado. Agradecimento especial à $\mathrm{OP} 2 \mathrm{~B}$ por todo o tempo disponibilizado para a realização das matérias.

A Deus, por tudo. 


\section{RESUMO}

Controle preditivo baseado em modelos (MPC) é uma técnica de controle amplamente utilizada na indústria de processos químicos. Por outro lado, o método de identificação em subespaços (SID) tem se mostrado uma alternativa eficiente para os métodos clássicos de identificação de sistemas. Pela combinação dos conceitos de MPC e SID, surgiu, no final da década de 90, uma nova técnica de controle, denominada controle preditivo com enfoque em subespaços (SPC). Essa técnica também é conhecida como controle preditivo orientado a dados. Ela substitui por um único passo as três etapas do projeto de um MPC: a identificação do modelo, o cálculo do observador de estados e a construção das matrizes de predição. Este trabalho tem como principal objetivo revisar estudos feitos na área de SPC, aplicar esse método em sistemas típicos da indústria química e propor novos algoritmos. São desenvolvidos três algoritmos de excitação interna para o método SPC, que permitem gerar dados persistentemente excitantes enquanto um controle mínimo do processo é garantido. Esses algoritmos possibilitam aplicar identificação em malha fechada, na qual o modelo do controlador SPC é reidentificado utilizando dados previamente excitados. Os controladores SPC e SPC com excitação interna são testados e comparados ao MPC por meio de simulações em dois processos distintos. O primeiro consiste em uma coluna debutanizadora de uma unidade de destilação, para a qual são disponibilizados dois modelos lineares referentes a pontos de operação diferentes. O segundo é um reator de polimerização de estireno com dinâmica não linear, cujo modelo fenomenológico é conhecido. Os resultados dos testes indicam que o SPC é mais suscetível a ruídos de medição. Entretanto, verifica-se que esse controlador corrige perturbações nos set-points das variáveis controladas mais rapidamente que o MPC. Simulações realizadas para o SPC com excitação interna mostram que os algoritmos propostos neste trabalho excitam 0 sistema satisfatoriamente, de modo que modelos mais precisos são obtidos na reidentificação com os dados excitados.

Palavras-chave: Identificação em subespaços. Identificação em malha fechada. Controle preditivo. Controle preditivo com enfoque em subespaços. Controle preditivo orientado a dados. Controle de processos. 


\begin{abstract}
Model Predictive Control (MPC) technology is widely used in chemical process industries. Subspace identification (SID) on the other hand has proven to be an efficient alternative for classical system identification methods. Based on the results from MPC and SID, it was developed in the late 90's a new control approach, called Subspace Predictive Control (SPC). This approach is also known as data-driven predictive control. In this new method, one single operation replaces the three steps in a MPC controller design: system identification, the state observer design and the predictor matrices construction. The aim of this work is to review studies in the field of SPC, to apply this technology to typical systems of chemical industry and to propose new algorithms. It is developed three internal excitation algorithms for the SPC method, which allow the system to be persistently excited while a minimal control of the process is still guaranteed. These algorithms enable the application of closedloop identification, where the SPC controller model is re-identified using the previously excited data. The SPC controller and the SPC controller with internal excitation are tested through simulation for two different processes. The first one is a debutanizer column of a distillation unit for which two linear models corresponding to two different operating points are available. The second one is a non-linear system consisting of a styrene polymerization reactor. A phenomenological model is provided for this system. Tests results indicate that SPC is more susceptible to measurement noises. However, it is noticed that SPC controller corrects perturbations on set-points faster than MPC. Simulations for the SPC with internal excitation show that the proposed algorithms sufficiently excite the system, in the sense that more precise models are obtained from the re-identification with excited data.
\end{abstract}

Keywords: Subspace identification. Closed-loop identification. Predictive control. Subspace predictive control. Data-driven predictive control. Process control. 


\section{LISTA DE ILUSTRAÇÕES}

Figura 3.1 - Representação esquemática do procedimento de identificação de sistemas.

Figura 3.2 - Representação em blocos de um sistema em malha fechada. 35

Figura 3.3 - Representação esquemática dos métodos de identificação SID e PEM. 38

Figura 3.4 - Interpretação de uma projeção ortogonal..........................................46

Figura 3.5 - Interpretação de uma projeção obliqua. ..........................................48

Figura 3.6 - Etapas de um algoritmo de identificação em subespaços......................49

Figura 3.7 - Resumo do algoritmo robusto de identificação em subespaços. ...........56

Figura 3.8 - Fluxograma da coluna debutanizadora da refinaria RPBC. ................57

Figura 3.9 - Comparação da resposta ao degrau dos diferentes modelos da coluna debutanizadora.

Figura 3.10 - Dados amostrados pelo experimento de identificação A. Planta sem ruído.

Figura 3.11 - Dados amostrados pelo experimento de identificação E. Ruído aleatório de variância 0.01 adicionado à saída da planta.

Figura 3.12 - Validação cruzada do modelo identificado pelo método PEM para os dados coletados no experimento A. Planta sem ruído.

Figura 3.13 - Determinação da ordem do modelo no método SID para os dados do experimento A. Planta sem ruído.

Figura 3.14 - Validação cruzada do modelo identificado pelo método SID para os dados coletados no experimento $A$. Planta sem ruído.

Figura 3.15 - Comparação das respostas ao degrau do sistema real e dos modelos obtidos via PEM e SID para os dados do experimento A. Planta sem ruído.

Figura 3.16 - Validação cruzada do modelo identificado pelo método PEM para os dados coletados no experimento C. Ruído de variância 0.0005 adicionado à saída da planta.

Figura 3.17- Determinação da ordem do modelo no método SID para os dados do experimento C. Ruído de variância 0.0005 adicionado à saída. 
Figura 3.18 - Validação cruzada do modelo identificado pelo método SID para os dados coletados no experimento C. Ruído de variância 0.0005 adicionado à saída da planta.

Figura 3.19 - Comparação das respostas ao degrau do sistema real e dos modelos obtidos via PEM e SID para os dados do experimento C. Ruído de variância 0.0005 adicionado à saída da planta.

Figura 3.20 - Determinação da ordem do modelo no método SID para os dados do experimento B. Ruído de variância 0.0001 adicionado à saída da planta.

Figura 3.21 - Validação cruzada do modelo identificado pelo método SID para os dados coletados no experimento B. Ruído de variância 0.0001 adicionado à saída da planta.

Figura 3.22 - Comparação das respostas ao degrau do sistema real e do modelo obtido via SID para os dados do experimento B. Ruído de variância 0.0001 adicionado à saída da planta.

Figura 4.1 - Projeto de um controlador MPC tradicional e do controlador SPC........73

Figura 4.2 - Comparação das respostas ao degrau do sistema real da coluna debutanizadora e dos modelos de predição obtidos pela identificação com dados sem ruído.

Figura 4.3 - Comparação das respostas ao degrau do sistema real da coluna debutanizadora e dos modelos de predição obtidos pela identificação com dados com ruído de variância 0.001 .

Figura 4.4 - Comparação das respostas ao degrau do sistema real da coluna debutanizadora e dos modelos de predição obtidos a partir do modelo em espaço de estados.

Figura 4.5 - Análise da influência de $M$ no SPC para o caso de controlador com modelo perfeito.

Figura 4.6 - Análise da influência de $M$ no SPC para o caso de controlador com erro no modelo devido à mudança no ponto de operação.

Figura 4.7 - Análise da influência de $M$ no SPC para o caso de controlador com erro no modelo devido à identificação com dados com ruído. .98

Figura 4.8 - Comparação entre SPC e MPC. Caso de controladores com modelos perfeitos e processo sem ruído. 
Figura 4.9 - Comparação entre SPC e MPC. Caso de controladores com modelos perfeitos e distúrbio pontual na entrada do processo.

Figura 4.10 - Comparação entre SPC e MPC. Caso de controladores com modelos perfeitos e distúrbio pontual na saída do processo.

Figura 4.11 - Comparação entre SPC e MPC. Caso de controladores com modelos perfeitos e ruído aleatório de variância 0.01 na entrada do processo. . 101

Figura 4.12 - Comparação entre SPC e MPC. Caso de controladores com modelos perfeitos e ruído aleatório de variância 0.01 na saída do processo......102

Figura 4.13 - Comparação entre SPC e MPC. Caso de controladores com erro nos modelos e processo sem ruído.

Figura 4.14 - Controlador SPC com reidentificação do modelo sem excitação adicional. 105

Figura 5.1 - Comportamento das variáveis do processo controlado pelo SPC com excitação direta na entrada.

Figura 5.2 - Função objetivo do controlador SPC com excitação direta na entrada.

Figura 5.3 - Controlador SPC com excitação direta na entrada e cálculo das direções menos excitadas a cada 200 minutos.

Figura 5.4 - Controlador SPC com excitação no incremento da entrada e cálculo das direções menos excitadas a cada 200 minutos.

Figura 5.5 - Controlador SPC com cálculo do valor de referência e cálculo das direções menos excitadas a cada 200 minutos.

Figura 5.6 - Controlador SPC com excitação interna. Análise do número de direções menos excitadas

Figura 5.7 - Função objetivo do controlador SPC com excitação interna. Análise do número de direções menos excitadas.

Figura 5.8 - Controlador SPC com excitação interna. Análise do nível de excitação.

Figura 5.9 - Função objetivo do controlador SPC com excitação interna. Análise do nível de excitação.

Figura 5.10 - Controlador SPC com excitação interna. Análise do peso da excitação em relação ao controle.

Figura 5.11 - Função objetivo do controlador SPC com excitação interna. Análise do peso da excitação em relação ao controle. 
Figura 5.12 - Controlador SPC com excitação interna. Análise do intervalo de cálculo das direções

Figura 5.13 - Função objetivo do controlador SPC com excitação interna. Análise do intervalo de cálculo das direções.

Figura 5.14 - Comparação entre MPC e SPC com reidentificação do modelo para o sistema da coluna debutanizadora. Visão Geral.

Figura 5.15 - Comparação entre MPC e SPC com reidentificação do modelo para o sistema da coluna debutanizadora. Ambos os controladores com modelo inicial (Período de 0 a 3500 minutos).

Figura 5.16 - Comparação entre MPC e SPC com reidentificação do modelo para o sistema da coluna debutanizadora. SPC com condição de excitação ativa (Período de 3500 a 7000 minutos). 135

Figura 5.17 - Comparação entre MPC e SPC com reidentificação do modelo para o sistema da coluna debutanizadora. SPC com modelo reidentificado (Período de 7000 a 9000 minutos).

Figura 6.1 - Representação esquemática do reator CSTR de polimerização de estireno.

Figura 6.2 - Comparação entre as respostas ao degrau do sistema do reator de polimerização e do modelo linear.

Figura 6.3 - Comparação entre as respostas ao degrau do reator de polimerização em diferentes pontos de operação.

Figura 6.4 - Influência do horizonte de dados passados no SPC para o sistema do reator de polimerização.

Figura 6.5 - Comparação entre MPC e SPC com reidentificação do modelo para o sistema do reator de polimerização. Visão Geral.

Figura 6.6 - Comparação entre MPC e SPC com reidentificação do modelo para o sistema do reator de polimerização. Ambos os controladores com modelo inicial (Período de 0 a 200 horas).

Figura 6.7 - Comparação entre MPC e SPC com reidentificação do modelo para o sistema do reator de polimerização. Controlador SPC com condição de excitação ativa (Período de 200 a 1400 horas).

Figura 6.8 - Comparação entre MPC e SPC com reidentificação do modelo.

Controlador SPC com modelo reidentificado (Período de 1400 a 1500 horas). 
Figura 6.9 - Comparação das respostas ao degrau dos modelos de predição do sistema do reator de polimerização identificados com diferentes números de dados....... 150 


\section{LISTA DE TABELAS}

Tabela 3.1 - Nomenclatura das estruturas de modelos polinomiais. 39

Tabela 3.2 - Variáveis manipuladas do sistema da coluna debutanizadora. 58

Tabela 3.3 - Variáveis controladas do sistema da coluna debutanizadora .58

Tabela 3.4 - Descrição dos experimentos de coleta de dados para a coluna debutanizadora.

Tabela 3.5 - Resumo dos testes de identificação e validação feitos para a coluna debutanizadora.

Tabela 3.6 - Resumo dos testes para análise de influência do parâmetro $i$ no método SID

Tabela 3.7 - Resumo dos testes para análise de influência do parâmetro $n x$ no método SID

Tabela 4.1 - Sintonia base do controlador SPC

Tabela 4.2 - Resumo dos estudos realizados para análise do parâmetro $M$ no SPC.

Tabela 5.1 - Sintonia do controlador SPC com excitação interna 113

Tabela 5.2 - Sintonia base do controlador SPC para análise dos parâmetros de sintonia.

Tabela 6.1 - Condições operacionais do reator de polimerização no estado estacionário.

Tabela 6.2 - Parâmetros do processo de polimerização no estado estacionário....141

Tabela 6.3 - Variáveis manipuladas do sistema do reator de polimerização.

Tabela 6.4 - Variáveis controladas do sistema do reator de polimerização.

Tabela 6.5 - Sintonia do controlador SPC para o sistema do reator de polimerização. Valores relativos ao estado estacionário.

Tabela 6.6 - Sintonia adicional de excitação do controlador SPC para o sistema do reator de polimerização. 


\section{LISTA DE ABREVIATURAS E SIGLAS}

$\begin{array}{ll}\text { AIBN } & \text { 2,2-azo-bis-iso-butironitrila } \\ \text { CVA } & \text { Canonical Variate Analysis } \\ \text { DMC } & \text { Dynamic Matrix Control } \\ \text { FIR } & \text { Finite Impulse Response } \\ \text { GLP } & \text { Gás Liquefeito de Petróleo } \\ \text { LQG } & \text { Linear Quadratic Gaussian } \\ \text { LTI } & \text { Linear Time Invariant } \\ \text { MIMO } & \text { Multi-Input Multi-Output } \\ \text { MOESP } & \text { Multivariable Output Error State Space } \\ \text { MPC } & \text { Model Predictive Control } \\ \text { N4SID } & \text { Subspace State Space System IDentification } \\ \text { PEM } & \text { Prediction Error Method } \\ \text { Petrobras } & \text { Petróleo Brasileiro S/A } \\ \text { PRBS } & \text { Pseudo Random Binary Sequence } \\ \text { QP } & \text { Quadratic Programming } \\ \text { SID } & \text { Subspace Identification } \\ \text { SISO } & \text { Single-Input Single-Output } \\ \text { SPC } & \text { Subspace Predictive Control } \\ \text { SVD } & \text { Singular Value Decomposition } \\ \text { RPBC } & \text { Refinaria Presidente Bernardes Cubatão }\end{array}$




\section{LISTA DE SÍMBOLOS}

matriz dos estados do modelo em espaço de estados matriz das entradas do modelo em espaço de estados matriz das saídas do modelo em espaço de estados matriz da realimentação direta do modelo em espaço de estados matriz de bloco triangular inferior de Toeplitz determinística número de blocos de linhas nas matrizes de Hankel matriz identidade de dimensão $x$ número de colunas nas matrizes de blocos de Hankel função custo do controlador preditivo convencional parcela da função custo do SPC referente a excitação função custo do SPC com excitação interna instante de tempo atual matriz de ganhos do observador de estados número de saídas do sistema matriz das entradas futuras do modelo de predição em subespaços matriz dos dados passados do modelo de predição em subespaços número de entradas do sistema horizonte do modelo de predição em subespaços número de dados amostrados ordem do denominador do modelo ARX ordem do numerador do modelo ARX número de direções menos excitadas consideradas no SPC horizonte de controle no controlador preditivo horizonte de predição no controlador preditivo número de estados do modelo em espaço de estados peso do termo da excitação em relação ao controle matriz de pesos das saídas do controlador matriz de supressão das entradas do controlador 
matriz de covariância cruzada entre os ruídos $w$ e $v$ intervalo de cálculo das direções menos exitadas vetor das entradas do sistema vetor de entradas futuras do modelo de predição em subespaços vetor com as ações de controle calculadas pelo MPC vetor dos valores máximos para as entradas do sistema vetor dos valores mínimos para as entradas do sistema vetor de entradas passadas do modelo de predição em subespaços vetor das entradas no estado estacionário vetor das variações das entradas do sistema vetor com as variações das ações de controle calculadas pelo MPC vetor de máxima variação das entradas do sistema matriz de blocos de Hankel das entradas futuras matriz de blocos de Hankel das entradas passadas vetor do ruído de medição matriz de autocovariância do ruído de medição vetor do ruído do processo vetor de entradas e saídas passadas do modelo de predição matriz de autocovariância do ruído do processo matriz de blocos de Hankel das saídas e entradas passadas vetor de estados do modelo em espaço de estados seqüência de estados futuros seqüência de estados passados vetor das saídas do sistema valor de referência (set-point) da saída do sistema vetor de saídas futuras do modelo de predição em subespaços vetor de saídas passadas do modelo de predição em subespaços matriz de blocos de Hankel das saídas futuras matriz de blocos de Hankel das saídas passadas 


\section{Símbolos Gregos}

$\begin{array}{ll}\Delta & \text { variação de uma variável entre dois instantes de tempo } \\ \Delta_{i}^{d} & \text { matriz reversa de controlabilidade estendida } \\ \Gamma_{i} & \text { matriz de observabilidade estendida } \\ \mathcal{E} & \text { vetor do erro de predição } \\ \theta & \text { vetor de parâmetros do modelo usado no PEM } \\ \Pi_{A} & \text { operador de projeção ortogonal sobre o espaço de linhas de } A \\ \rho & \text { nível de excitação } \\ \varphi & \text { vetor de dados usado no PEM }\end{array}$

\section{Sobrescritos}

$\begin{array}{ll}* & \text { valor ótimo } \\ \wedge & \text { pseudo-inversa de Moore-Penrose } \\ a & \text { valor predito da variável } \\ a h & \text { modelo para processo sem ação direta } \\ d & \text { modelo para processo sem ação direta e horizontes independentes } \\ s & \text { componente determinístico } \\ T & \text { transposição de uma matriz }\end{array}$




\section{SUMÁRIO}

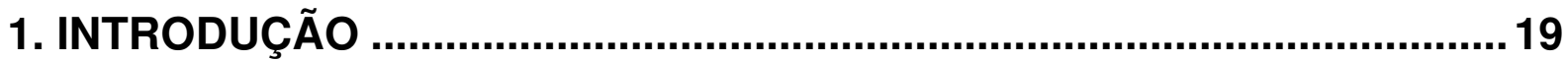

2. CONTROLE PREDITIVO BASEADO EM MODELOS ….......................... 25

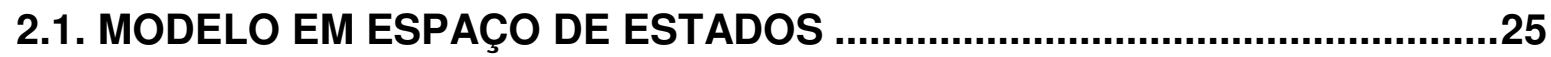

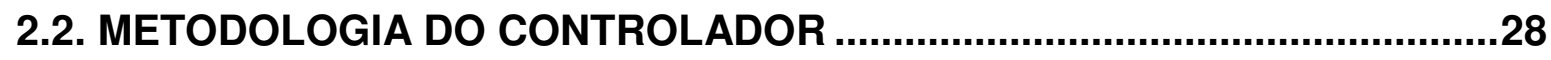

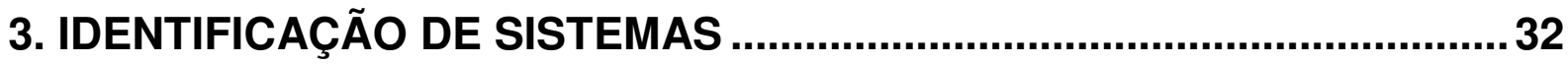

3.1. APRESENTAÇÃO DO PROBLEMA DE IDENTIFICAÇÃO …......................32

3.2. MÉTODOS DE IDENTIFICAÇÃO............................................................37

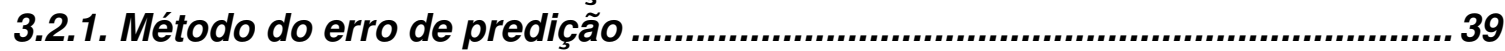

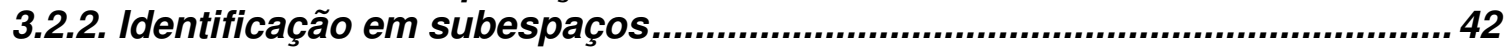

3.3. APLICAÇÃO DOS MÉTODOS DE IDENTIFICAÇÃO.................................57

3.3.1. Apresentação do sistema da coluna debutanizadora...................................5 57

3.3.2. Comparações entre os métodos PEM e SID.............................................. 60

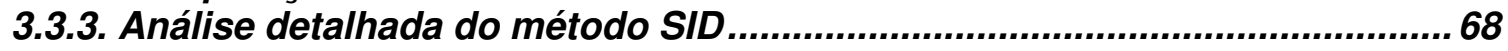

4. CONTROLE PREDITIVO COM ENFOQUE EM SUBESPAÇOS ................72

4.1. MODELO DE PREDIÇÃO EM SUBESPAÇOS...........................................74

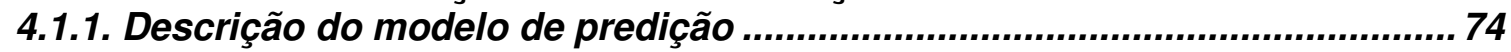

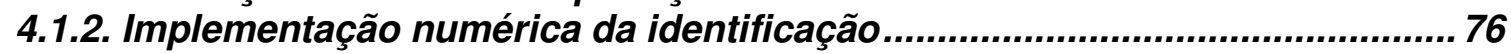

4.1.3. Relação com modelos em espaço de estados.............................................. 81

4.1.4. Obtenção do modelo de predição incremental na entrada ............................. 84

4.2. METODOLOGIA DO CONTROLADOR ....................................................86

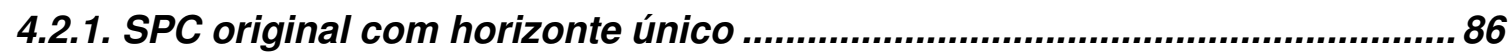

4.2.2. SPC para processo sem ação direta e com horizontes independentes....... 88

4.3. SIMULAÇÕES E ANÁLISES ................................................................94

4.3.1. Influência do horizonte de dados passados no controlador.......................... 94

4.3.2. Comparação entre SPC e MPC................................................................... 98

4.3.3. Análise do SPC com reidentifação em malha fechada ................................. 104

5. SPC COM EXCITAÇÃO INTERNA...................................................106

5.1. CONTROLADOR COM EXCITAÇÃO DIRETA NA ENTRADA ...................107

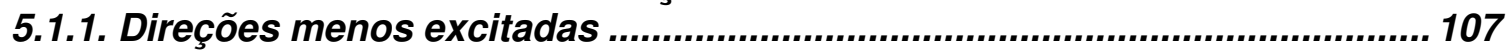

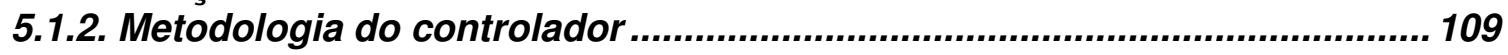

5.1.3. Cálculo do estado estacionário................................................................ 116

5.2. CONTROLADOR COM EXCITAÇÃO NO INCREMENTO DA ENTRADA ..118

5.3. CONTROLADOR COM CÁLCULO DO VALOR DE REFERÊNCIA.............121

5.4. SIMULAÇÕES E ANÁLISES …..........................................................125

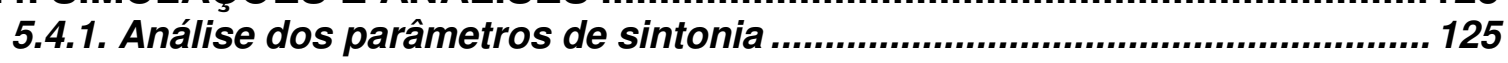

5.4.2. Simulação do SPC com reidentificação em malha fechada......................... 133 
6. APLICAÇÃO DO SPC PARA UM SISTEMA NÃO-LINEAR

6.1. APRESENTAÇÃO DO SISTEMA …................................................137

6.1.1. Descrição do modelo fenomenológico....................................................137

6.1.2. Linearização do sistema .......................................................................... 142

6.1.3. Resposta do processo em diferentes pontos de operação........................... 143

6.2. SINTONIA DO CONTROLADOR SPC..................................................144

6.3. SIMULAÇÃO DO SPC COM REIDENTIFICAÇÃO DO MODELO ...............146

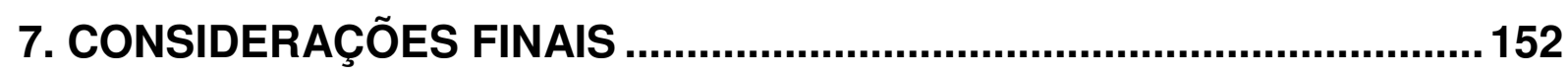

7.1. CONCLUSÕES ...........................................................................152

7.2. SUGESTÕES DE CONTINUIDADE ...................................................154

REFERÊNCIAS ............................................................................... 156 


\section{INTRODUÇÃO}

Controle preditivo baseado em modelos (MPC, do inglês Model Predictive Control) refere-se a uma classe de algoritmos de controle que utilizam um modelo explícito para prever o comportamento do sistema controlado. A cada período de controle, um problema de otimização é resolvido, no qual ações de controle futuras são calculadas. A primeira ação de controle é então enviada à planta. No próximo instante de tempo, os parâmetros do modelo são atualizados com dados obtidos por medição na planta e o problema de otimização é recalculado. Essa estratégia também pode ser chamada de controle com horizonte móvel, pois a cada período de controle, o horizonte de tempo considerado é transladado em uma unidade.

O MPC é amplamente utilizado na indústria de processos químicos. Seu sucesso pode ser justificado por alguns aspectos interessantes, como a facilidade de impor restrições nas entradas e saídas do processo e a possibilidade de se obter ganhos econômicos com uma operação ótima da planta (RODRIGUES; ODLOAK, 2000). Além disso, a formulação MPC é essencialmente multivariável e sistemas com dinâmica lenta e tempo morto, características muito comuns em processos químicos, são facilmente tratados.

O desempenho do MPC é extremamente dependente da qualidade do modelo utilizado. Um controle rigoroso das variáveis do processo requer um modelo preciso. Há basicamente duas formas de se obter um modelo: modelagem ou enfoque analítico e identificação de sistemas. A modelagem consiste na descrição fenomenológica do processo por meio de equações baseadas nos princípios básicos da Física. Esse método, além de ser muito trabalhoso, gera modelos complexos que necessitam de muitos parâmetros. Já a identificação de sistema é baseada em experimentos. Sinais de entrada e de saída do sistema são registrados e um modelo é inferido através da analise dos dados coletados (GARCIA, 2008). Os modelos obtidos dessa maneira geralmente possuem uma faixa de validade mais limitada, porém são mais adequados para o uso em MPC, pois são mais simples e consideram apenas as variáveis globais do sistema. 
A técnica mais tradicional de identificação de sistemas é o método do erro de predição (PEM, do inglês Prediction Error Method), o qual busca obter o modelo que minimize o erro entre as saídas preditas e as saídas medidas na planta. Nas duas últimas décadas um novo enfoque, chamado identificação em subespaços (SID, do inglês Subspace Identification) tem atraído muita atenção. O SID engloba uma série de algoritmos de identificação que se baseiam em conceitos da teoria de sistemas, geometria e álgebra linear numérica (FAVOREEL; DE MOOR; VAN OVERSCHEE, 2000). Um grande número de aplicações do método SID para sistemas simulados e reais foram apresentados na literatura, sendo que essa técnica já alcançou um grau significativo de maturidade e aceitação.

Entre as vantagens do SID, pode-se destacar a sua simplicidade e robustez numérica, além da capacidade de tratar sistemas multivariáveis sem nenhum esforço adicional. Enquanto no PEM há uma parametrização prévia do sistema, no SID não é necessário nenhuma informação. O modelo é identificado no formato de espaço de estados, que é extremamente conveniente para o uso em MPC. O único parâmetro necessário, a ordem do modelo, é obtido durante o procedimento de identificação. Ao contrário do que geralmente ocorre no PEM, no SID não é necessário resolver problemas de otimização não-lineares. Em contra partida, os modelos obtidos via SID são sub-ótimos, no sentido que eles não são uma solução ótima de um problema de minimização do erro (VAN OVERSCHEE; DE MOOR, 1997).

Com base nos conceitos do MPC e do SID, surgiu, no final da década de 90, um novo método de controle preditivo que combina a identificação do modelo e o controle do processo em uma única etapa. A maneira clássica de controle consiste em primeiramente se obter um modelo paramétrico do sistema para posteriormente construir as matrizes de predição das saídas futuras usadas no controlador. No novo método, essas matrizes de predição são calculadas diretamente de dados passados da planta. Diversos nomes têm sido usados na literatura para referenciar essa nova técnica de controle. Por não utilizar um modelo paramétrico na forma tradicional, alguns autores adotaram o nome "model-free approach" (enfoque sem modelo). Outros adotaram o nome "data-driven predictive control" (controle preditivo orientado a dados). Favoreel e De Moor (1998) denominaram esse método de controle 
preditivo com enfoque em subespaços (SPC, do inglês Subspace Predictive Control), que será o nome usado nesta Dissertação.

A qualidade dos dados usados para identificação do modelo no MPC ou para o cálculo das matrizes de predição no SPC (que também podem ser consideradas como um tipo de modelo) é determinante para o desempenho desses controladores. Para se obter um bom modelo é necessário que os dados utilizados sejam persistentemente excitantes, ou seja, todos os modos dinâmicos de interesse do processo devem ser excitados. Em geral, quanto maior a amplitude do sinal de entrada, mais preciso é o modelo obtido (GARCIA, 2008). Porém, na prática, essa condição de excitação confronta com o objetivo de controle.

Após certo tempo de operação, os controladores preditivos podem sofrer uma queda no desempenho, devido à diminuição na precisão do modelo utilizado. Essa degradação do modelo pode ser explicada pela alteração nas características dinâmicas da planta, ocasionadas, por exemplo, por desgaste de equipamentos ou mudança das especificações do produto (BALLIN, 2008). Controladores de sistemas não-lineares que foram aproximados por modelos lineares e tiveram seus pontos de operação alterados também sofrem esse problema. Nesses casos, para resgatar o desempenho inicial do controlador é necessário fazer nova coleta de dados e reidentificação do sistema.

Tradicionalmente, a etapa de coletas de dados consiste em testes em malha aberta, nos quais as variáveis manipuladas são excitadas individualmente. Esse é um trabalho muito demorado e de elevado custo. Segundo Li e Georgakis (2008), testes desse tipo para um sistema com dinâmica na ordem de horas pode demorar semanas ou meses, dependendo do número de entradas e saídas do sistema. Além disso, por questões de segurança e/ou econômica, durante o experimento de identificação, é necessário manter as saídas do processo dentro de determinadas faixas. Assim, testes em malha aberta necessitam de monitoramento contínuo para que sejam realizadas correções manuais, caso alguma variável saia da faixa de operação. Por todos esses motivos é muito importante garantir a eficácia do procedimento de excitação do sistema e registro de dados. 
$\mathrm{Na}$ tentativa de resolver os problemas encontrados na identificação em malha aberta, surgiu a técnica de identificação em malha fechada, na qual o sistema permanece sob a ação de um controlador. Essa é uma área de pesquisa que tem recebido bastante atenção na última década. Uma característica importante dessa técnica é a redução das perturbações devida à ação do controlador. Além disso, testes desse tipo são mais fáceis de executar, pois aspectos de especificação de produto e de segurança e estabilidade do processo são tratados automaticamente, eliminando atuações manuais. Ainda, segundo Hjalmarsson; Gevers e De Bruyne (1996), para o propósito de controle, modelos de qualidade superior são obtidos a partir de testes em malha fechada.

Segundo Garcia (2008), a principal dificuldade com dados obtidos a partir de um processo em malha fechada é que o sistema pode se tornar não identificável, ou seja, pode ser impossível determinar todos os parâmetros do modelo. Esse problema pode ser contornado se a realimentação for suficientemente complexa. $O$ MPC com restrições, que tem características não lineares e variantes no tempo, atende esse requisito. Outra maneira de garantir a identificabilidade do sistema em malha fechada é adição de uma excitação externa à malha de controle (GUSTAVSSON; LJUNG; SÖDERSTRÖM, 1977). Esse sinal externo deve ser persistentemente excitante no intervalo de freqüência de interesse e independente dos distúrbios do processo (SOTOMAYOR; ODLOAK; MORO, 2009).

Com o intuito de garantir a persistência de excitação em malha fechada, surgiram novos enfoques para a identificação de processos controlados por MPC, que podem ser chamados de métodos com excitação interna (SOTOMAYOR; ODLOAK; MORO, 2009). Esses métodos se baseiam na adição de restrições de excitação no problema de otimização do MPC ou em modificações na função objetivo. A idéia é permitir que o sistema seja suficientemente excitado ao mesmo tempo em que um controle mínimo é mantido, garantido aspectos de segurança e especificação de produto. Além disso, muitos dos métodos propostos podem ser utilizados na forma de controle adaptativo, no qual a reidentificação do sistema é feita a cada período de amostragem. 
Como exemplos de algoritmos de identificação em malha fechada com excitação interna pode-se citar os trabalhos de Shouche et al. (1998), Aggelogiannaki e Sarimveis (2006) e Ballin (2008). Todos esses algoritmos trabalham com a adição de restrições de excitação no problema de otimização do controlador. Uma grande desvantagem é que essas restrições são não-lineares, tornando o problema de otimização não-convexo. Dessa maneira, não é mais possível resolvê-lo através de programação quadrática (QP, do inglês Quadratic Programming), uma técnica bem consolidada e com diversos algoritmos robustos desenvolvidos.

No enfoque do SPC, Hallouzi e Verhaegen (2008) propuseram um controlador adaptativo com excitação interna que em cada instante de amostragem as matrizes de predição são recalculadas. Nesse controlador uma condição de excitação é adicionada na função objetivo do problema de otimização de forma a manter a sua convexidade. Parâmetros de sintonia foram adicionados permitindo que o trade-off entre excitação e controle seja ajustado.

A técnica de controle SPC traz características interessantes, como realizar as etapas de identificação e parametrização do controlador preditivo em uma única etapa. Além disso, não é necessária a utilização de um observador de estados, como por exemplo, o filtro de Kalman, necessário em modelos em espaço de estados usados no MPC. Vale ainda ressaltar que o SPC surgiu a partir do MPC e SID, técnicas de sucesso na indústria. Porém, esse método de controle é relativamente novo, com apenas 10 anos de existência, e poucas aplicações foram realizadas. Assim, surge a motivação para se estudar o SPC e aplicá-lo em sistemas típicos da indústria de processos químicos. Nota-se também que ainda há bastante espaço para novos desenvolvimentos nessa área.

O principal objetivo deste trabalho é revisar estudos feitos na área de SPC, aplicar essa técnica de controle em sistemas típicos da indústria química e propor novos algoritmos. Em especial, baseado no SPC adaptativo de Hallouzi e Verhaegen (2008), são desenvolvidos três novos algoritmos de excitação interna para o método SPC, nos quais a persistência de excitação dos dados gerados é garantida. Este trabalho ainda possui dois objetivos secundários. O primeiro é revisar as duas principais técnicas de identificação de sistemas, PEM e SID, e aplicá-las em um 
sistema real. O segundo consiste em analisar as diferenças e semelhanças entre o MPC e o SPC.

Esta Dissertação está estruturada da seguinte forma: no Capítulo 2 uma breve introdução ao MPC é realizada. No Capítulo 3 são descritos alguns aspectos importantes da área de identificação de sistemas e os métodos PEM e SID. $O$ sistema de uma coluna debutanizadora da Refinaria Presidente Bernardes Cubatão (RPBC) da Petróleo Brasileiro S/A (Petrobras) é apresentado. A esse sistema são aplicados ambos os métodos de identificação e realiza-se uma análise detalhada dos resultados.

No Capítulo 4 é introduzido o SPC. O modelo de predição usado nesse controlador é apresentado e são mostradas as possíveis formas de obtê-lo. Analisa-se a influência dos parâmetros de sintonia do SPC e compara-se o desempenho desse controlador com o desempenho do MPC. No Capítulo 5 são propostos três algoritmos de excitação interna para o método SPC. Uma análise detalhada desses algoritmos é realizada através de simulações com o sistema da coluna debutanizadora. No Capitulo 6 descreve-se um sistema não-linear referente a um reator de polimerização. O controlador SPC e um dos algoritmos de excitação interna desenvolvidos no Capítulo 5 são então aplicados a esse sistema. Por fim, no Capítulo 7 são apresentadas as conclusões deste trabalho e sugestões de continuidade. 


\section{CONTROLE PREDITIVO BASEADO EM MODELOS}

Controle preditivo baseado em modelos (MPC) refere-se a uma classe de algoritmos computacionais de controle que utilizam um modelo explícito para prever o comportamento das saídas futuras da planta. Essa tecnologia é amplamente utilizada na indústria de processos químicos e geralmente é a técnica padrão usada em aplicações de controle avançado (GONZÁLEZ; ADAM; MARCHETTI, 2008).

O MPC surgiu no final da década de 70, quando Cutler e Ramaker (1979) propuseram o chamado DMC (Dynamic Matrix Control). Desde então diversos artigos foram publicados e essa área obteve grande desenvolvimento. Boas revisões de MPC podem ser encontradas em Rawlings (2000) e Maciejowski (2002). Qin e Badgwell (2003) apresentam um bom resumo das tecnologias MPC disponíveis comercialmente.

Existem basicamente três enfoques utilizados em MPC. Cada enfoque adota uma estrutura de modelo. As possíveis estruturas são: resposta ao degrau ou resposta ao impulso (FIR, do inglês Finite Impulse Response); função de transferência; e modelo em espaço de estados (WANG; YOUNG, 2006). Os primeiros algoritmos de controle preditivo baseavam-se em resposta ao degrau ou FIR. Por esse motivo essa é a técnica mais empregada nos controladores comerciais. Entretanto, no meio acadêmico, o enfoque mais utilizado atualmente, e que tem se mostrado mais eficiente, é o MPC baseado em modelo em espaço de estados. Esse será o enfoque usado neste trabalho.

\subsection{MODELO EM ESPAÇO DE ESTADOS}

O estado de um sistema dinâmico é um conjunto de variáveis que com o conhecimento delas em determinado instante de tempo, em conjunto com a informação das entradas futuras, determina completamente o comportamento futuro 
do sistema (ODLOAK, 2008). Essas variáveis são geralmente agrupadas em um vetor denominado vetor de estados. Esse vetor funciona como uma ponte de interligação entre o passado e o futuro.

Um sistema linear invariante no tempo (LTI, do inglês Linear Time Invariant) pode ser representado por um modelo em espaço de estados com intervalos de tempo discretos da seguinte forma:

$$
\begin{aligned}
& x(k+1)=A x(k)+B \Delta u(k) \\
& y(k)=C x(k)+D \Delta u(k)
\end{aligned}
$$

sendo:

$x(k+1) \in R^{n x}$ vetor de estados no instante de tempo $k+1$;

$x(k) \in R^{n x} \quad$ vetor de estados no instante $k$;

$\Delta u(k)=u(k)-u(k-1) \in R^{m}$ vetor das variações das entradas no instante $k$;

$$
\begin{array}{ll}
u(k) \in R^{m} & \text { vetor das entradas no instante } k ; \\
y(k) \in R^{l} & \text { vetor das saídas no instante } k ; \\
A \in R^{n x \times n x} & \text { matriz dos estados do modelo; } \\
B \in R^{n x \times m} & \text { matriz das entradas do modelo; } \\
C \in R^{l \times n x} & \text { matriz das saídas do modelo; } \\
D \in R^{l \times m} & \text { matriz da realimentação direta do modelo; } \\
n x & \text { número de estados do modelo; } \\
m & \text { número de entradas do sistema; } \\
l & \text { número de saídas do sistema; } \\
k & \text { instante de tempo atual. }
\end{array}
$$

Duas observações importantes podem ser feitas acerca desse modelo:

1) $O$ modelo apresentado é incremental em relação às entradas. Essa representação foi escolhida, pois no MPC o uso das entradas na forma incremental é uma condição necessária para um controle livre de off-set. 
2) Para processos típicos da indústria química a matriz $D$ é nula, ou seja, não há realimentação direta das entradas nas saídas;

Diversas formas de se obter as matrizes $A, B, C$ e $D$ do modelo (2.1)-(2.2) foram propostas na literatura, como por exemplo, Wang e Young (2006) e Rodrigues e Odloak (2000). Na maioria desses métodos, as matrizes são obtidas a partir de um modelo prévio, como função de transferência ou equação de diferença. A identificação em subespaços, que será detalhada no capítulo seguinte, é uma forma de se obter as matrizes do modelo em espaço de estados diretamente de dados coletados da planta.

Quando um modelo em espaço de estados é utilizado para predição $n$ passos à frente, como é o caso em aplicações de MPC, é necessário, em cada período de amostragem, atualizar o estado predito com o estado atual da planta. Seja o estado predito pelo modelo no instante $k+1$ :

$$
\hat{x}(k+1)=A \hat{x}(k)+B \Delta u(k)
$$

Se o modelo usado for perfeito e as condições iniciais forem exatas, o estado predito será idêntico ao estado real da planta, não sendo necessário nenhum tipo de atualização. Porém, na prática, isso não acontece. Nos casos em que os componentes do vetor de estados são propriedades medidas, a atualização é realizada facilmente por uma simples substituição do estado predito pelo estado real disponível. Nos demais casos é preciso utilizar um estimador de estados. A reconstrução do estado é feita com base no erro de predição da saída $(y-C \hat{x}-D \Delta u)$ da seguinte forma:

$$
\hat{x}(k+1 \mid k)=A \hat{x}(k \mid k-1)+B \Delta u(k)+K[y(k)-C \hat{x}(k \mid k-1)-D \Delta u(k)]
$$

A notação $\hat{x}(j \mid s)$ é usada para indicar a predição do estado no instante $j$ com base nas medidas disponíveis até o instante $s$. O parâmetro $K$ é uma matriz de ganhos que deve ser propriamente escolhida, sendo determinada pelo estimador de estados. 
Um dos estimadores de estados mais eficientes e utilizados é o Filtro de Kalman. Ele adota como critério para o calculo da matriz de ganhos $(K)$ a minimização da covariância do erro da estimativa do estado, sendo o erro da estimativa do estado a diferença entre o estado medido e o estado predito pelo modelo. Odloak (2008) demonstra que a matriz de ganhos do Filtro de Kalman pode ser obtida pela resolução das seguintes equações:

$$
\begin{aligned}
& K=A P C^{T}\left(V+C P C^{T}\right)^{-1} \\
& P=A P A^{T}-A P C^{T}\left(V+C P C^{T}\right)^{-1} C P A^{T}+W
\end{aligned}
$$

sendo $P$ a covariância do erro da estimativa do estado e $W$ e $V$ as matrizes de covariância dos ruídos do processo e de medição, respectivamente. A equação (2.6) deve ser resolvida por um método iterativo. As matrizes $W$ e $V$ podem ser estimadas por métodos de identificação estocástica de sistemas, porém esse procedimento pode ser difícil de ser realizado. Por simplificação, em aplicações de controle é usual considerar $W$ e $V$ como parâmetros de sintonia, que são ajustados manualmente.

\subsection{METODOLOGIA DO CONTROLADOR}

Neste trabalho será utilizado o MPC de horizonte finito com modelo em espaço de estados na forma incremental, representado por (2.1)-(2.2). Será considerado apenas o caso de sistemas sem ação direta da entrada na saída $(D=0)$. A cada período de amostragem o controlador resolve o seguinte problema de otimização:

$$
\min _{\Delta u_{k}} J_{k}=\sum_{j=1}^{n p}\left(y(k+j \mid k)-y^{S P}\right)^{T} Q\left(y(k+j \mid k)-y^{S P}\right)+\sum_{j=0}^{n c-1} \Delta u(k+j \mid k)^{T} R \Delta u(k+j \mid k)
$$

sujeito a:

$$
\begin{aligned}
& -\Delta u_{\max } \leq \Delta u(k+j \mid k) \leq \Delta u_{\max }, \quad j=0, \ldots, n c-1 \\
& u_{\min } \leq u(k+j \mid k) \leq u_{\max }, \quad j=0, \ldots, n c-1
\end{aligned}
$$


sendo:

$Q \in R^{l \times l} \quad$ matriz de pesos das saídas;

$R \in R^{m \times m} \quad$ matriz de supressão das entradas;

$y^{S P} \in R^{l} \quad$ vetor de referência (set-point) das saídas;

$u_{\min } \in R^{m} \quad$ valores mínimos para as entradas;

$u_{\max } \in R^{m} \quad$ valores máximos para as entradas;

$\Delta u_{\max } \in R^{m} \quad$ variação máxima das entradas em cada instante;

np horizonte de predição;

nc horizonte de controle;

$J_{k} \quad$ função custo do controlador no instante $k$;

$\Delta u_{k}=\left[\begin{array}{llll}\Delta u(k \mid k)^{T} & \Delta u(k+1 \mid k)^{T} \quad \cdots \quad \Delta u(k+n c-1 \mid k)^{T}\end{array}\right]^{T}$.

O problema acima pode ser escrito na forma de programação quadrática (QP). Para isso, deve-se colocar as predições das saídas futuras em um único vetor. O próximo passo consiste em determinar a relação desse vetor com as variáveis do problema $\left(\Delta u_{k}\right)$ e com o modelo em espaço de estados.

A partir das equações (2.1) e (2.2) do modelo e considerando $D=0$, pode-se escrever:

$$
\begin{aligned}
& y(k+1 \mid k)=C x(k+1 \mid k) \\
& =C A x(k)+C B \Delta u(k \mid k) \\
& y(k+2 \mid k)=C A x(k+1 \mid k)+C B \Delta u(k+1 \mid k) \\
& =C A^{2} x(k)+C A B \Delta u(k \mid k)+C B \Delta u(k+1 \mid k) \\
& y(k+3 \mid k)=C A^{3} x(k)+C A^{2} B \Delta u(k \mid k)+C A B \Delta u(k+1 \mid k)+C B \Delta u(k+2 \mid k) \\
& y(k+j \mid k)=C A^{j} x(k)+C A^{j-1} B \Delta u(k \mid k)+C A^{j-2} B \Delta u(k+1 \mid k)+\ldots+C B \Delta u(k+j-1 \mid k)
\end{aligned}
$$

No MPC de horizonte finito considera-se que a partir do instante $k+n c-1$ as entradas permanecem constantes, ou seja, $\Delta u(k+n c \mid k)=\Delta u(k+n c+1 \mid k)=\cdots=0$. Então se pode escrever: 


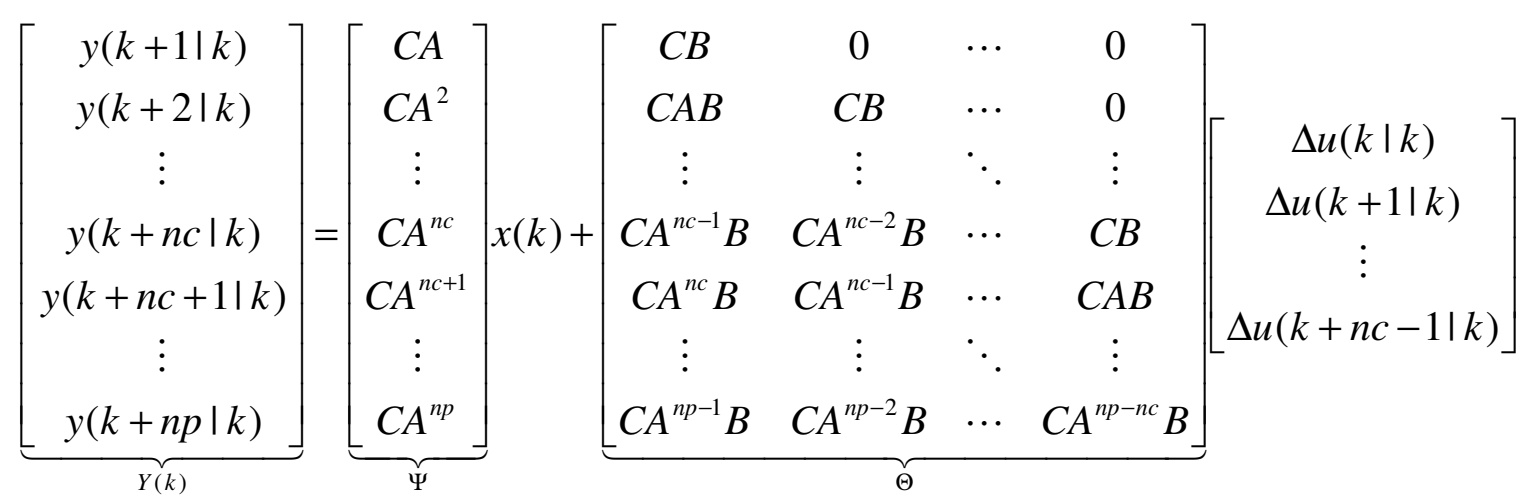

Assim, o vetor de predições das saídas futuras pode ser escrito na forma:

$$
Y(k)=\Psi x(k)+\Theta \Delta u_{k}
$$

Também é necessário representar o vetor com as entradas futuras $\left(u_{k}\right)$, presente na restrição (2.9), em função do vetor das variações das entradas futuras $\left(\Delta u_{k}\right)$. Por definição tem-se:

$$
\begin{aligned}
& u(k \mid k)=\Delta u(k \mid k)+u(k-1) \\
& u(k+1 \mid k)=\Delta u(k+1 \mid k)+\Delta u(k \mid k)+u(k-1) \\
& \quad \vdots \\
& u(k+n c-1 \mid k)=\Delta u(k+n c-1 \mid k)+\ldots+\Delta u(k \mid k)+u(k-1)
\end{aligned}
$$

As equações acima podem ser escritas na seguinte forma:

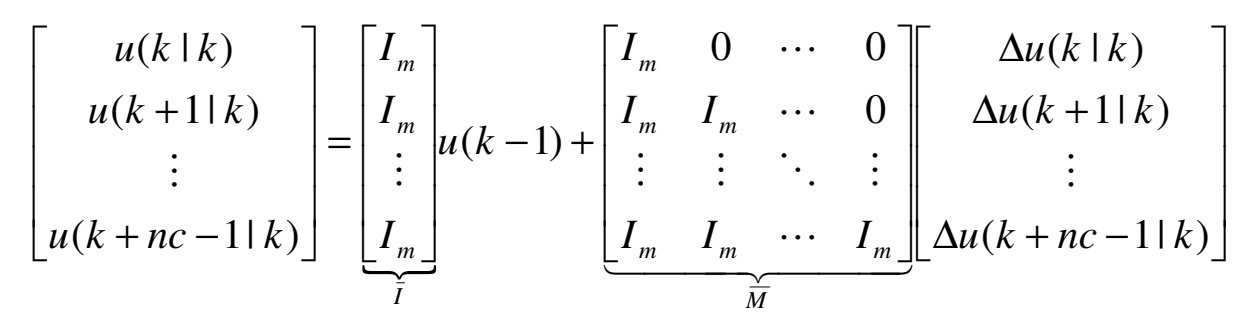

O vetor de entradas futuras pode então ser escrito como:

$$
u_{k}=\bar{I} u(k-1)+\bar{M} \Delta u_{k}
$$

sendo $u_{k}=\left[\begin{array}{llll}u(k \mid k)^{T} & u(k+1 \mid k)^{T} & \cdots & u(k+n c-1 \mid k)^{T}\end{array}\right]^{T}$. 
Utilizando as equações (2.10) e (2.11), o problema de otimização representado pela função objetivo (2.7) e pelas restrições (2.8) e (2.9) pode ser escrito como:

$$
\min _{\Delta u_{k}} \quad J_{k}=\Delta u_{k}{ }^{T} H_{1} \Delta u_{k}+2 c_{f 1}{ }^{T} \Delta u_{k}+c_{1}
$$

sujeito a: $A_{\text {ineq } 1} \Delta u_{k} \leq b_{\text {ineq1 }}$

sendo:

$$
\begin{aligned}
& H_{1}=\Theta^{T} \bar{Q} \Theta+\bar{R} ; \\
& c_{f 1}^{T}=\left[\Psi x(k)-Y_{s p}\right]^{T} \bar{Q} \Theta ; \\
& c_{1}=\left[\Psi x(k)-Y_{s p}\right]^{T} \bar{Q}\left[\Psi x(k)-Y_{s p}\right] \text {; }
\end{aligned}
$$

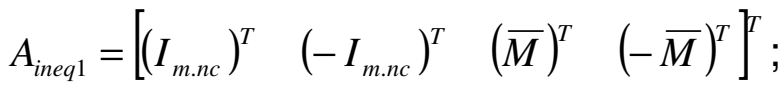

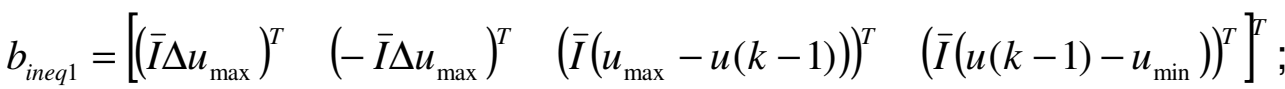

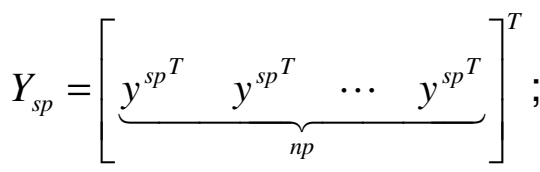

$$
\begin{aligned}
& \bar{Q}=\left[\begin{array}{cccc}
Q & 0 & \cdots & 0 \\
0 & Q & \cdots & 0 \\
\vdots & \vdots & \ddots & \vdots \\
0 & 0 & \cdots & Q
\end{array}\right] ; \\
& \bar{R}=\left[\begin{array}{cccc}
R & 0 & \cdots & 0 \\
0 & R & \cdots & 0 \\
\vdots & \vdots & \ddots & \vdots \\
0 & 0 & \cdots & R
\end{array}\right] ;
\end{aligned}
$$

Após o cálculo das ações de controle futuras pela resolução do problema de otimização (2.7)-(2.9), a primeira ação de controle $(u(k))$ é adicionada ao sistema. As saídas do processo são então medidas e com essas novas informações, no próximo período de amostragem, todo o procedimento é repetido. 


\section{IDENTIFICAÇÃO DE SISTEMAS}

Um controle de processo bem sucedido tem como pré-requisito uma boa identificação. A qualidade do modelo utilizado impacta profundamente 0 desempenho do controlador. Porém, deve-se ter em mente que um modelo nunca pode ser aceito como uma descrição final e verdadeira do sistema (LJUNG, 1999). Assim, o objetivo de uma identificação é encontrar um modelo que seja uma descrição boa o suficiente dos aspectos do sistema relevantes para determinado propósito, como por exemplo, o controle.

A seguir, na seção 3.1, serão apresentados os principais aspectos que devem ser considerados na identificação de sistemas. Na seção 3.2 são descritas as duas principais classes de algoritmos de identificação, o método do erro de predição (PEM) e a identificação em subespaços (SID). Na seção 3.3 esses métodos são aplicados em um sistema típico da indústria química.

\subsection{APRESENTAÇÃO DO PROBLEMA DE IDENTIFICAÇÃO}

Pode-se definir a identificação de sistemas como a área de modelagem matemática a partir de dados experimentais (ZHU, 2001). Mais detalhadamente, o procedimento de identificação consiste em, a partir de dados amostrados, determinar o melhor modelo (representação do sistema) dentro de uma classe de modelos, com base em determinado critério. Assim, é possível destacar três componentes básicos envolvidos na identificação de sistemas: o conjunto de dados, a classe de modelos e o critério para estimação.

O conjunto de dados é normalmente obtido a partir de testes projetados especificamente para o fim de identificação, de forma que ele contenha toda a informação necessária. A definição da classe de modelos é uma etapa que normalmente utiliza informações prévias do sistema. Por fim, o critério utilizado para 
a escolha do melhor modelo consiste nos algoritmos de identificação propriamente ditos. Após a identificação, é preciso verificar se o modelo é bom o suficiente para a aplicação pretendida, o que é feito na etapa de validação. Assim, o procedimento de identificação pode ser resumido nos seguintes passos (ZHU, 2001):

1. Experimento de identificação

2. Escolha da estrutura do modelo

3. Estimação do modelo

4. Validação do modelo

A figura abaixo mostra de forma esquemática o procedimento de identificação de sistemas:

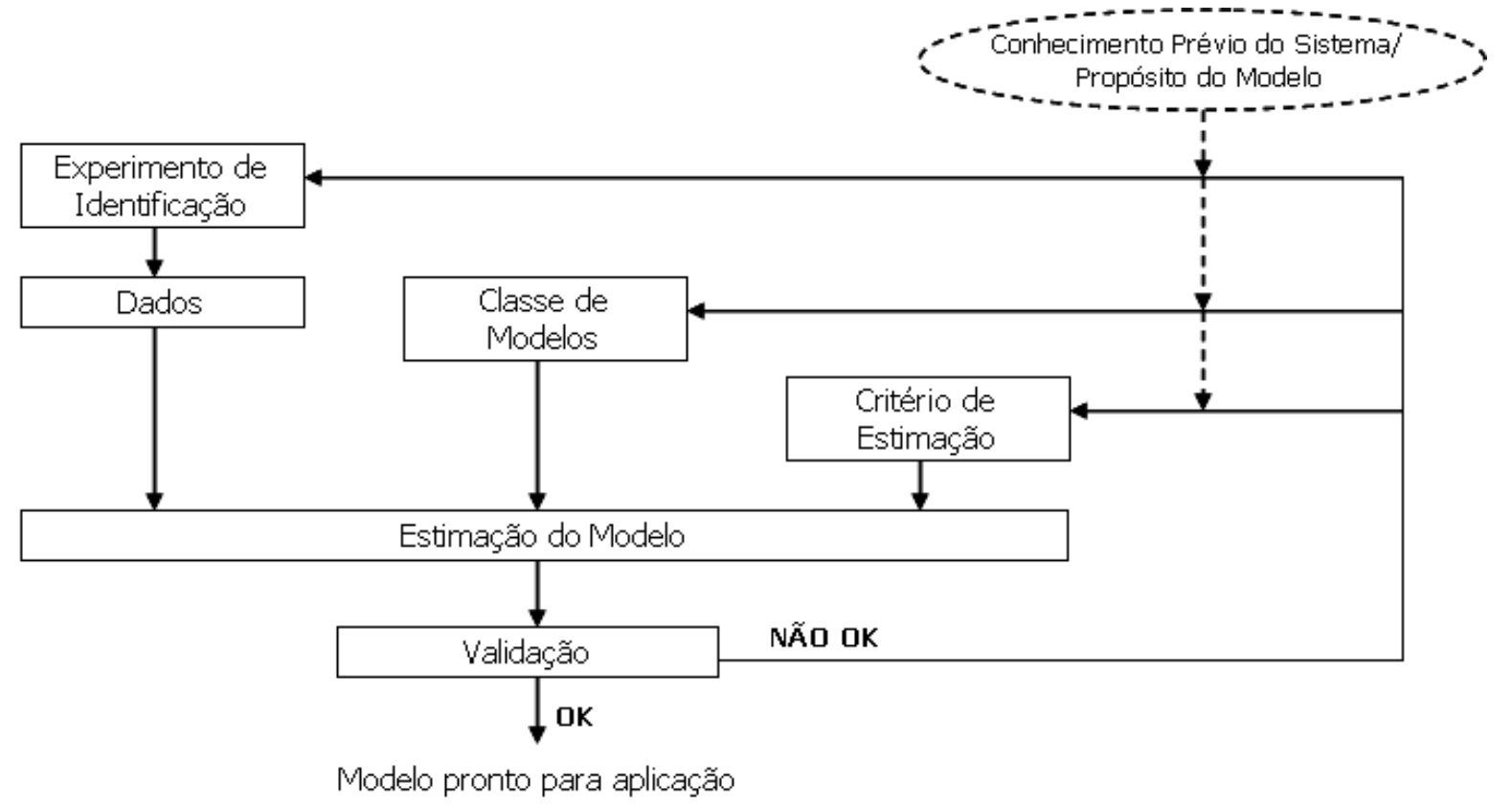

Figura 3.1 - Representação esquemática do procedimento de identificação de sistemas.

O experimento de identificação tem como objetivo excitar o sistema e coletar informações relevantes da dinâmica do processo. Ele deve ser projetado cuidadosamente, pois essa é a etapa crítica da identificação, com duração longa e custos elevados. Dada a sua importância, alguns autores, como Garcia (2008), consideram o projeto do experimento uma etapa separada. Uma série de escolhas deve ser feita, incluindo o sinal de entrada, a taxa de amostragem, duração do experimento e se os testes serão realizados em malha aberta ou fechada. A escolha das variáveis de entrada e de saída é uma tarefa complexa e não será detalhada 
aqui, pois como o intuito da identificação é o controle, essas variáveis devem ser as mesmas utilizadas no controlador. Cada uma das escolhas apontadas acima será brevemente discutida nos próximos parágrafos.

Segundo Li e Georgakis (2008), projetar bons sinais de entrada é o primeiro e muito importante passo para se obter um bom modelo. Devem ser considerados os seguintes aspectos: a forma, a amplitude e o espectro (conteúdo no domínio da freqüência). A amplitude deve ser escolhida de modo que a razão entre o sinal e o ruído seja razoavelmente alta, mas que ao mesmo tempo não traga perturbação excessiva que possa retirar o processo da região desejada, saindo, por exemplo, da região linear do sistema (ZHU, 2001). Como resultado final, o sinal projetado deve ser persistentemente excitante de ordem suficientemente alta.

A taxa de amostragem refere-se ao intervalo de tempo usado para realizar o registro dos dados. Diversas regras práticas para a escolha do período de amostragem podem ser encontradas em Garcia (2008) e Zhu (2001). No caso de identificação de sistemas para controle de processos, o período de amostragem usado deve ser o mesmo utilizado pelo controlador.

$\mathrm{Na}$ visão da identificação, os experimentos devem ter a maior duração possível. Porém, na visão do controle, a duração deve ser mínima, devido às perturbações adicionais geradas. Segundo Zhu (2001), os experimentos duram, geralmente, na faixa de 8 a 16 vezes o período de estabilização do sistema. $O$ experimento precisa ser longo o suficiente para ser possível retirar, via média, o efeito dos distúrbios não medidos. Além disso, muitos métodos de identificação baseiam-se na hipótese de que o número de dados disponíveis tende ao infinito.

Uma última escolha refere-se à identificação em malha aberta ou fechada. Normalmente, os sinais de excitação são aplicados nas variáveis de entrada, quando em malha aberta, e nos set-points das variáveis de saída, quando em malha fechada. O método clássico utilizado na indústria é a identificação em malha aberta. Porém, conforme já apresentado na introdução deste trabalho, o método de identificação em malha fechada é mais adequado para o propósito de controle. 
A identificação em malha fechada reduz as perturbações do processo pela ação do controlador, além de ser um experimento mais fácil de executar e normalmente de menor duração. A desvantagem desse método é que a entrada do processo é correlacionada com os distúrbios não medidos, sempre que a ação do controlador não é nula (FORSSELL; LJUNG, 1999). Problemas de identificabilidade podem surgir, porém eles são superados pela adição de excitação externa persistentemente excitante ou pelo uso de controladores com realimentação complexa, como o MPC, que possui características não-lineares e variantes no tempo.

Abaixo está a representação em blocos de um sistema em malha fechada:

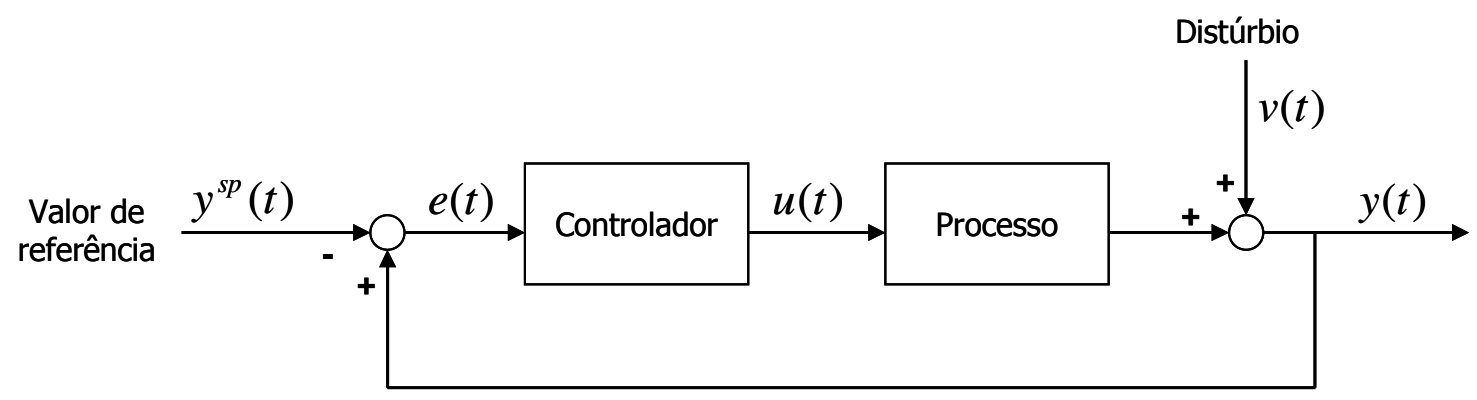

Figura 3.2 - Representação em blocos de um sistema em malha fechada.

As técnicas de identificação em malha fechada podem ser classificadas em método direto, método indireto e método conjunto de entrada e saída. O método direto é análogo à identificação em malha aberta, com uso da entrada $(u(t))$ e da saída $(y(t))$ do processo. O método indireto utiliza o valor de referência $\left(y^{s p}(t)\right)$ no lugar da entrada do processo $(u(t))$. Por último, o método conjunto de entrada e saída considera a entrada $(u(t))$ e a saída $(y(t))$ como variáveis dependentes do valor de referência $\left(y^{s p}(t)\right)$ e da perturbação $(v(t))$. Com as descrições acima e observando a Figura 3.2, percebe-se que os métodos indireto e conjunto de entrada e saída requerem um conhecimento prévio do controlador. Normalmente, assume-se que o controlador possui certa estrutura linear e invariante no tempo (LTI). Por esse motivo, eles não são aplicáveis a sistemas controlados por MPC (SOTOMAYOR; ODLOAK; MORO, 2009).

Dependendo da tecnologia utilizada, a etapa do projeto do experimento é reduzida, às vezes limitando-se apenas à escolha da duração do experimento. Testes 
realizados em malha fechada por meio de controladores preditivos com excitação interna, por exemplo, já possuem a forma do sinal de excitação definida. Nesses casos, parâmetros de sintonia podem ser adicionados para dar flexibilidade ao experimento, como permitir o ajuste da amplitude da excitação.

Muitos trabalhos foram publicados na área de projetos de experimentos para controle, como: Bombois et al. (2006) e Forssell e Ljung (2000). Hjalmarsson (2005) trata esse aspecto especificamente para o caso de testes em malha fechada. Zhu (2001) descreve de forma bem didática um procedimento prático para se obter um bom conjunto de dados. Esse procedimento inclui testes preliminares para se determinar algumas informações iniciais acerca da dinâmica do processo, seguido de um teste final para a coleta dos dados.

Com os dados amostrados, a próxima etapa é a determinação da estrutura do modelo, que pode ser mais ampla ou mais específica. Nesse momento, deve-se definir se será usada uma abordagem linear ou não-linear e se o modelo será do tipo paramétrico ou não-paramétrico. É preciso ainda decidir sobre o formato da representação matemática. No caso de modelos lineares paramétricos, eles podem ser representados, por exemplo, por função de transferência, equações de diferenças ou modelo em espaço de estados. É importante ressaltar que as escolhas feitas nessa etapa restringem os algoritmos de identificação que poderão ser aplicados.

A etapa de estimação do modelo corresponde à aplicação de um algoritmo de identificação. Segundo Forssell e Ljung (1999), esses algoritmos podem ser divididos em três tipos: método do erro de predição (PEM), método de identificação em subespaços (SID) e análise espectral e de correlação. Os dois primeiros referemse à identificação de modelos paramétricos, que será enfocada neste trabalho, enquanto que o último é usado para a identificação de modelos não paramétricos. Para modelos paramétricos, o PEM é a técnica clássica utilizada. O SID surgiu em meados da década de 90 e tem se mostrado uma boa alternativa para o PEM. Esses métodos serão detalhados na próxima seção. 
Após a estimação do modelo, é preciso verificar a sua qualidade, o que é feito na etapa de validação. Essa etapa deve ser dedicada à avaliação do modelo com respeito ao seu propósito: predição, simulação, controle, etc. (FORSSELL; LJUNG, 2000). Existem diversas maneiras de avaliar o modelo. A forma mais natural é por meio da comparação entre a simulação do modelo obtido e os dados medidos do processo real (GARCIA, 2008). Quando os dados da planta usados na simulação são diferentes dos dados usados na estimação do modelo, esse método é denominado validação cruzada, que é um dos mais importantes métodos empregados na indústria.

Muitos índices são utilizados para se obter uma análise quantitativa do modelo, sendo que um bom resumo sobre esse tópico pode ser encontrado em Garcia (2008). A grande maioria dos índices trata o trade-off existente entre o ajuste do modelo aos dados e o aumento da complexidade do modelo resultante da inclusão de termos. Esses índices são baseados em modelos polinomiais usados no PEM e não são aplicáveis ao SID, que utiliza modelo em espaço de estados e não possui nenhuma parametrização prévia. Um índice que pode ser medido para qualquer modelo é o índice de ajuste (FIT), dado por:

$$
\operatorname{FIT}(\%)=100 \cdot\left(\frac{1-\|\hat{y}(t)-y(t)\|}{\|y(t)-\bar{y}\|}\right)
$$

sendo $\hat{y}(t)$ o valor predito pelo modelo, $y(t)$ o valor real do sistema e $\bar{y}$ o valor médio dos dados amostrados do sistema.

\subsection{MÉTODOS DE IDENTIFICAÇÃO}

Nesta seção serão apresentados os dois principais métodos de identificação de sistemas para a classe de modelos paramétricos, lineares e invariantes no tempo: o método do erro de predição e o método de identificação em subespaços. 
O método clássico de identificação é o método do erro de predição (PEM), que se baseia na resolução de problemas de otimização, muitas vezes não-lineares. Além do fato de ser necessária uma parametrização prévia do sistema, a natureza iterativa de muitos algoritmos requer estimativas iniciais precisas (ZHU, 2001).

Uma boa alternativa ao PEM é o método de identificação em subespaços (SID). Ele considera o modelo no formato em espaço de estados, eliminando o problema de parametrização prévia. O único parâmetro necessário, a ordem do modelo, é obtido durante o procedimento de identificação. Além disso, ele se baseia em conceitos da álgebra linear, resultando em algoritmos numericamente simples e estáveis. Ainda, esse método trata sistemas multivariáveis com a mesma facilidade que trata sistemas com única entrada e saída. Em contra partida, a precisão na estimação de modelos via SID não é tão boa quanto via PEM, em termos da variância das estimativas. Outra desvantagem é que quando aplicado para dados amostrados em malha fechada, o método SID tradicional estima modelos com bias, mesmo se as condições de identificabilidade são atendidas para o método PEM (QIN; LIN; LJUNG, 2005). Na tentativa de contornar esse problema, muitos estudos têm sido feitos no desenvolvimento de algoritmos SID para identificação em malha fechada, como por exemplo, Wang e Qin (2006) e Katayama e Tanaka (2007).

O SID utiliza o conceito de estado de um sistema dinâmico no contexto da identificação, enquanto o PEM é baseado em um enfoque de entrada-saída (VAN OVERSCHEE; DE MOOR, 1996). As diferenças entre esses dois métodos podem ser representadas conforme a figura abaixo:

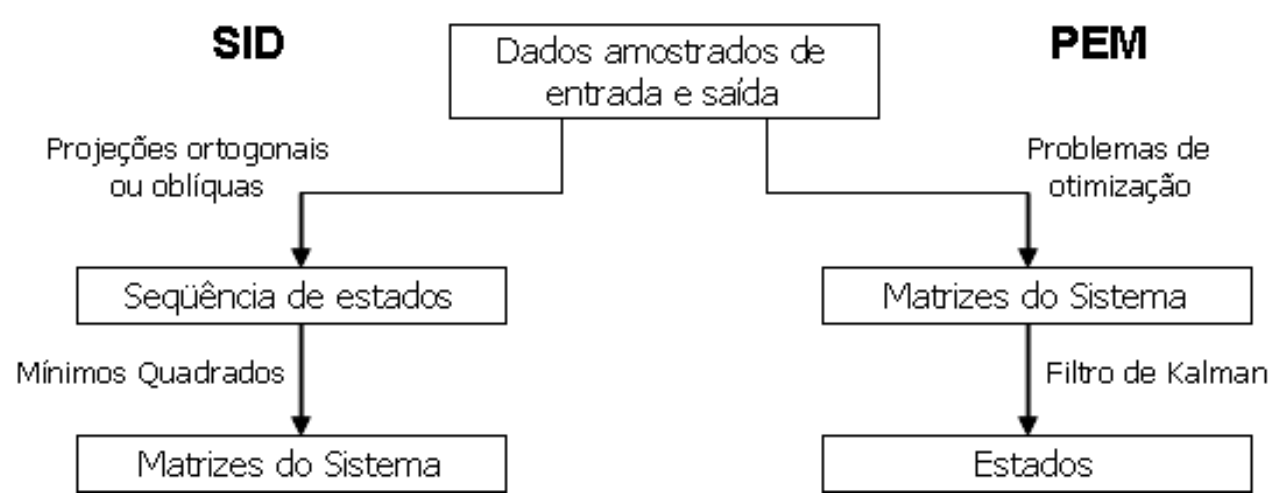

Figura 3.3 - Representação esquemática dos métodos de identificação SID e PEM. 


\subsubsection{Método do erro de predição}

O método do erro de predição (PEM) refere-se a uma classe de algoritmos de identificação que se baseia na simples idéia de que, na ausência de distúrbios, o melhor modelo é aquele que gera o menor erro de predição, sendo o erro de predição a diferença entre a saída medida e a saída calculada pelo modelo (MICCHI; PANNOCCHIA, 2008).

O primeiro passo da identificação via PEM é a escolha da estrutura do modelo. Normalmente são utilizados modelos no formato de função de transferência discreta ou equação de diferenças equivalente. Considera-se que a saída do processo $(y(t))$ é dependente da entrada $(u(t))$ e do ruído $(e(t))$. Uma estrutura generalizada desse tipo de modelo pode ser escrita como (HUANG; KADALI, 2008):

$$
A\left(q^{-1}\right) y(t)=\frac{B\left(q^{-1}\right)}{F\left(q^{-1}\right)} u(t)+\frac{C\left(q^{-1}\right)}{D\left(q^{-1}\right)} e(t)
$$

sendo $A, B, C, D$ e $F$ polinômios de ordem a ser escolhida. O componente $q^{-1}$ é usado para denotar o operador de retrocesso (backward shift operator). Dependendo dos polinômios utilizados, a estrutura do modelo recebe um nome específico, conforme é mostrado na Tabela 3.1 (GARCIA, 2008):

Tabela 3.1 - Nomenclatura das estruturas de modelos polinomiais.

\begin{tabular}{cc}
\hline Polinômios usados & Nome estrutura do Modelo \\
\hline B & FIR \\
\hline A, B & ARX \\
\hline A, B, C & ARMAX \\
\hline A, C & ARMA \\
\hline A, , B, D & ARARX \\
\hline A, B, C, D & ARARMAX \\
\hline B, F & OE (output error) \\
\hline B, C, D, F & BJ (Box-Jenkins)
\end{tabular}

Uma estrutura simples e bastante utilizada é a ARX. Essa será a estrutura utilizada neste trabalho e por isso o método PEM será exemplificado para esse caso. Considere que o sistema é representado por um modelo ARX do tipo: 


$$
y(t)=\frac{B\left(q^{-1}\right)}{A\left(q^{-1}\right)} u(t)+\frac{1}{A\left(q^{-1}\right)} e(t)
$$

A equação acima também pode ser escrita na forma de equação de diferenças:

$$
y(t)+a_{1} y(t-1)+\ldots+a_{n_{a}} y\left(t-n_{a}\right)=b_{1} u(t-1)+\ldots+b_{n_{b}} u\left(t-n_{b}\right)+e(t)
$$

Por fim, a equação (3.4) pode ser escrita na forma de regressão linear:

$$
y(t)=\varphi(t) \theta+e(t)
$$

sendo $\varphi(t)=\left[\begin{array}{llllll}-y(t-1) & \cdots & -y\left(t-n_{a}\right) & u(t-1) & \cdots & u\left(t-n_{b}\right)\end{array}\right] \quad 0$ vetor de dados $\mathrm{e}$ $\theta=\left[\begin{array}{llllll}a_{1} & \cdots & a_{n_{a}} & b_{1} & \cdots & b_{n_{b}}\end{array}\right]^{T}$ o vetor de parâmetros.

Uma vez escolhida a estrutura do modelo, é preciso escolher as ordens dos polinômios da equação (3.2), que no caso do modelo ARX são representadas pelos parâmetros $n_{a}$ e $n_{b}$ da equação (3.4). No procedimento de identificação, o modelo real do processo não é conhecido e deseja-se obter uma estimação dos parâmetros do modelo. Para a estrutura ARX pode-se escrever a saída predita pelo modelo estimado como:

$$
\hat{y}(t)=\varphi(t) \hat{\theta}
$$

O erro de predição é definido da seguinte maneira:

$$
\varepsilon(t)=y(t)-\hat{y}(t)
$$

$\mathrm{Na}$ identificação, diversos dados são utilizados e, portanto pode-se calcular diversos erros de predição. Assim é necessário definir um critério de melhor ajuste para a estimação dos parâmetros. Alguns possíveis critérios são: minimização do somatório dos erros, minimização dos valores absolutos dos erros, minimização dos quadrados dos erros e minimização do máximo desvio (GARCIA, 2008). Um dos critérios mais usados é minimizar os quadrados dos erros. Esse método é conhecido como o 
método dos mínimos quadrados. Os parâmetros estimados pelo PEM com esse critério são solução do seguinte problema de otimização:

$$
\theta^{*}=\arg \min _{\theta} \sum_{i=1}^{N}(\varepsilon(i))^{2}
$$

sendo $\theta^{*}$ o vetor de parâmetros estimado pelo método dos mínimos quadrados e $N$ o número de dados usados na identificação.

Para o caso ARX, pode-se formar um sistema de $P$ equações, sendo $P=N-\max \left(n_{a}, n_{b}\right)+1$, a partir da seqüência de $N$ dados medidos do processo:

$$
\left[\begin{array}{c}
y\left(n_{a}+1\right) \\
y\left(n_{a}+2\right) \\
\vdots \\
y\left(n_{a}+P\right)
\end{array}\right]=\left[\begin{array}{c}
\varphi\left(n_{a}+1\right) \\
\varphi\left(n_{a}+2\right) \\
\vdots \\
\varphi\left(n_{a}+P\right)
\end{array}\right]\left[\begin{array}{c}
a_{1} \\
\vdots \\
a_{n_{a}} \\
b_{1} \\
\vdots \\
b_{n_{b}}
\end{array}\right]+\left[\begin{array}{c}
\varepsilon\left(n_{a}+1\right) \\
\varepsilon\left(n_{a}+2\right) \\
\vdots \\
\varepsilon\left(n_{a}+P\right)
\end{array}\right]
$$

sendo:

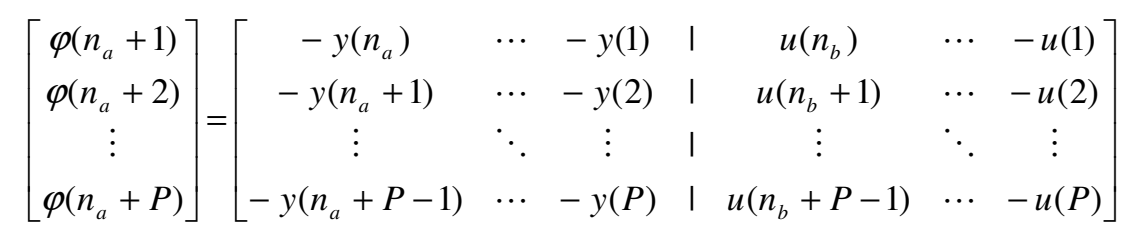

Deve-se ter $N>>n_{a}+n_{b}$. Pode-se escrever a equação (3.9) na forma compacta:

$$
Y=X \theta+\mathrm{E}
$$

A solução do problema dos mínimos quadrados apresentado na equação (3.8) para o modelo ARX é então dada por:

$$
\theta^{*}=\left(X^{T} X\right)^{-1} X^{T} Y
$$


É importante ressaltar que toda a descrição do método PEM foi realizada para o caso SISO (do inglês, Single-Input Single-Output). Para um sistema MIMO (do inglês, Multi-Input Multi-Output) pode-se descrever um procedimento análogo, no qual o erro de predição a cada instante passa a ser um vetor.

\subsubsection{Identificação em subespaços}

O método de identificação em subespaços (SID) surgiu no início da década de 90, como uma alternativa aos métodos clássicos de identificação para sistemas lineares e invariantes no tempo. Ele se baseia em conceitos da álgebra linear, o que the garante simplicidade e robustez numérica. No SID é considerado que o sistema com $m$ entradas e $l$ saídas é representado por um modelo em espaço de estados da seguinte forma:

$$
\begin{aligned}
& x(k+1)=A x(k)+B u(k)+w(k) \\
& y(k)=C x(k)+D u(k)+v(k)
\end{aligned}
$$

com

$$
E\left(\left[\begin{array}{c}
w(p) \\
v(p)
\end{array}\right]\left[\begin{array}{ll}
w(q)^{T} & v(q)^{T}
\end{array}\right]\right)=\left[\begin{array}{cc}
W & S \\
S^{T} & V
\end{array}\right] \delta_{p q} \geq 0
$$

Esse modelo é análogo ao representado pelas equações (2.1)-(2.2), porém é posicional na entrada. Os vetores $v(k) \in R^{l}$ e $w(k) \in R^{n x}$ são sinais não medidos e são chamados de ruído de medição e ruído do processo, respectivamente. As matrizes $W \in R^{n x \times n x}, S \in R^{n x \times l}$ e $V \in R^{l \times l}$ são matrizes de covariância dos ruídos $v(k)$ e $w(k)$. O delta de Kronecker $\left(\delta_{p q}\right)$ da equação (3.14) é um índice que recebe valor zero, quando $p \neq q$, e recebe valor um, caso contrário.

O problema a ser resolvido pelo SID pode ser descrito como: dado um conjunto grande de medições da entrada $u(k)$ e da saída $y(k)$, geradas pelo sistema desconhecido (3.12)-(3.14), determinar a ordem $n x$ do sistema, as matrizes $A, B$, 
$C$ e $D$ relativas a uma transformação de similaridade e uma estimação das matrizes $W, S$ e $V$ (FAVORREL; DE MOOR; VAN OVERSCHEE, 2000).

A seguir serão descritos a notação utilizada e os principais conceitos geométricos usados na identificação em subespaços. Posteriormente é apresentada a idéia geral por trás dos algoritmos de identificação, seguida da descrição detalhada de um algoritmo SID robusto.

\subsubsection{Notação}

$\mathrm{Na}$ identificação em subespaços, o sistema (3.12)-(3.13) é dividido em um subsistema estocástico e outro determinístico. Isso é feito pela divisão do estado $x(k)$ e da saída $y(k)$ em seus componentes determinístico $\left(\bullet^{d}\right)$ e estocástico $\left(\bullet^{s}\right)$ :

$$
\begin{aligned}
& x(k)=x^{d}(k)+x^{s}(k) \\
& y(k)=y^{d}(k)+y^{s}(k)
\end{aligned}
$$

O subsistema determinístico descreve a influência da entrada determinística $u(k)$ na saída determinística $y^{d}(k)$ :

$$
\begin{aligned}
& x^{d}(k+1)=A x^{d}(k)+B u(k) \\
& y^{d}(k)=C x^{d}(k)+D u(k)
\end{aligned}
$$

O subsistema estocástico descreve a influência dos ruídos $v(k)$ e $w(k)$ na saída estocástica $y^{s}(k)$ :

$$
\begin{aligned}
& x^{s}(k+1)=A x^{s}(k)+w(k) \\
& y^{s}(k)=C x^{s}(k)+v(k)
\end{aligned}
$$


Realizando-se uma substituição recursiva nas equações do modelo em espaço de estados para dados em diferentes instantes, obtêm-se as seguintes equações de matrizes de dados de entrada e saída:

$$
\begin{aligned}
& Y_{p}=\Gamma_{i} X_{p}^{d}+H_{i}^{d} U_{p}+Y_{p}^{s} \\
& Y_{f}=\Gamma_{i} X_{f}^{d}+H_{i}^{d} U_{f}+Y_{f}^{s} \\
& X_{f}^{d}=A^{i} X_{p}^{d}+\Delta_{i}^{d} U_{p}
\end{aligned}
$$

Essas equações possuem um papel importante no SID e cada um de seus componentes está descrito a seguir:

- Matrizes de blocos de Hankel de entrada e de saída:

$$
U_{0 \mid 2 i-1} \stackrel{\operatorname{def}}{=}\left[\begin{array}{cccc}
u(0) & u(1) & \cdots & u(j-1) \\
u(1) & u(2) & \cdots & u(j) \\
\vdots & \vdots & \ddots & \vdots \\
u(i-1) & u(i) & \cdots & u(i+j-2) \\
\hline u(i) & u(i+1) & \cdots & u(i+j-1) \\
u(i+1) & u(i+2) & \cdots & u(i+j) \\
\vdots & \vdots & \ddots & \vdots \\
u(2 i-1) & u(2 i) & \cdots & u(2 i+j-2)
\end{array}\right]=\left[\frac{U_{0 \mid i-1}}{U_{i \mid 2 i-1}}\right] \stackrel{\operatorname{def}}{=}\left[\frac{U_{p}}{U_{f}}\right]
$$

Na matriz acima, o número de blocos de linhas $(i)$ é definido pelo usuário e deve ser maior que a ordem do sistema $(n x)$. O número de blocos de coluna $(j)$ é calculado de forma que todos os $N$ dados disponíveis sejam usados, ou seja, $j=N-2 i+1$. O subscrito " $p$ " significa passado e o subscrito " $f$ " significa futuro. Essa notação é conveniente para a explicação de alguns conceitos de forma intuitiva, porém ela é vaga, visto que todos os dados usados na identificação são passados.

As matrizes de blocos de Hankel para as outras variáveis são definidas de maneira análoga. A matriz contendo as entradas $U_{p}$ e as saídas $Y_{p}$ é denominada $W_{p}$ :

$$
W_{p} \stackrel{\operatorname{def}}{=}\left[\begin{array}{c}
Y_{p} \\
U_{p}
\end{array}\right]
$$


- Seqüências de estados:

$$
\begin{aligned}
& X_{f} \stackrel{\text { def }}{=} X_{i} \stackrel{\text { def }}{=}\left[\begin{array}{llll}
x(i) & x(i+1) & \cdots & x(i+j-1)
\end{array}\right] \\
& X_{p} \stackrel{\text { def }}{=} X_{0} \stackrel{\text { def }}{=}\left[\begin{array}{llll}
x(0) & x(1) & \cdots & x(j-1)
\end{array}\right]
\end{aligned}
$$

Definição análoga é feita para as seqüências dos componentes estocásticos e determinísticos do vetor de estados.

- Matriz de observabilidade estendida $\Gamma_{i}$ :

$$
\Gamma_{i} \stackrel{\operatorname{def}}{=}\left[\begin{array}{c}
C \\
C A \\
\vdots \\
C A^{i-1}
\end{array}\right]
$$

- Matriz de bloco triangular inferior de Toeplitz determinística $H_{i}^{d}$ :

$$
H_{i}^{d} \stackrel{\text { def }}{=}\left[\begin{array}{cccc}
D & 0 & \cdots & 0 \\
C B & D & \cdots & 0 \\
\vdots & \vdots & \ddots & \vdots \\
C A^{i-2} B & C A^{i-3} B & \cdots & D
\end{array}\right]
$$

- Matriz reversa de controlabilidade estendida $\Delta_{i}^{d}$ :

$$
\Delta_{i}^{d} \stackrel{d e f}{=}\left[\begin{array}{lllll}
A^{i-1} B & A^{i-2} B & \cdots & A B & B
\end{array}\right]
$$

\subsubsection{Ferramentas da geometria}

A seguir serão apresentados os principais conceitos de geometria utilizados na identificação em subespaços. Eles serão descritos de forma independente do SID, focando nas definições existentes na álgebra linear. Ao longo dessa seção, considere três matrizes conhecidas com as seguintes dimensões: $A \in R^{p \times j}, B \in R^{q \times j}$ e $C \in R^{r \times j}$. Considere também que $j \geq \max (p, q, r)$. 
A projeção ortogonal de uma matriz sobre o espaço de linhas da matriz $B$ é representada pelo operador $\Pi_{B}$, que é definido como:

$$
\Pi_{B} \stackrel{\text { def }}{=} B^{T} .\left(B B^{T}\right)^{\dagger} . B
$$

onde $\bullet$ denota a pseudo-inversa de Moore-Penrose da matriz $\bullet$. A projeção do espaço de linhas da matriz $A$ sobre o espaço de linhas da matriz $B$, denotada por $A / B$, é definida como:

$$
A / B \stackrel{\text { def }}{=} A . \Pi_{B}=A B^{T} .\left(B B^{T}\right)^{\dagger} . B
$$

A Figura 3.4 mostra como a projeção ortogonal pode ser interpretada em um espaço de dimensão $j$. Observe que o resultado da projeção $A / B$ está dentro do espaço de linhas de $B$.

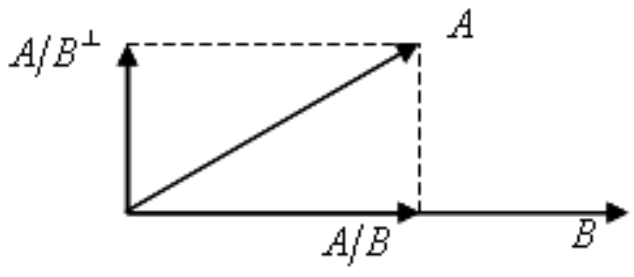

Figura 3.4 - Interpretação de uma projeção ortogonal.

Note que na Figura 3.4 aparece a projeção $A / B^{\perp}$, que representa a projeção do espaço de linhas da matriz $A$ sobre o complemento ortogonal do espaço de linhas da matriz $B$. Ela é definida como:

$$
A / B^{\perp} \stackrel{\text { def }}{=} A . \Pi_{B^{\perp}}
$$

sendo

$$
\Pi_{B^{\perp}}=I_{j}-\Pi_{B}
$$

A combinação das projeções $\Pi_{B}$ e $\Pi_{B^{\perp}}$ decompõe a matriz $A$ em duas matrizes cujos espaços de linhas são ortogonais: 


$$
A=A \cdot \Pi_{B}+A \cdot \Pi_{B^{\perp}}
$$

Alternativamente, as duas projeções determinam a matriz $A$ como uma combinação linear das linhas de $B$ e das linhas do complemento ortogonal de B:

$$
A=L_{B} \cdot B+L_{B^{\perp}} \cdot B^{\perp}
$$

sendo

$$
\begin{aligned}
& L_{B} . B \stackrel{\text { def }}{=} A / B \\
& L_{B^{\perp}} \cdot B^{\perp} \stackrel{\text { def }}{=} A / B^{\perp}
\end{aligned}
$$

A projeção obliqua do espaço de linhas da matriz $A$ alinhada ao espaço de linhas da matriz $B$ sobre o espaço de linhas da matriz $C$, denotada por $A /{ }_{B} C$, é definida como:

$$
A /{ }_{B} C \stackrel{\text { def }}{=}\left(A / B^{\perp}\right) \cdot\left(C / B^{\perp}\right)^{\dagger} \cdot C
$$

Ela pode ser interpretada como a decomposição da matriz $A$ em uma combinação linear de duas matrizes não ortogonais, $B$ e $C$, e do complemento ortogonal do espaço conjunto de $B$ e $C$. Pode-se então escrever:

$$
A=L_{B} \cdot B+L_{C} \cdot C+L_{B^{\perp}, C^{\perp}} \cdot\left[\begin{array}{l}
C \\
B
\end{array}\right]^{\perp}
$$

sendo

$$
A /{ }_{B} C \stackrel{\text { def }}{=} L_{C} \cdot C
$$

A Figura 3.5 ilustra uma projeção obliqua em um espaço de dimensão $j$. Outra maneira de interpretar a projeção obliqua $A /{ }_{B} C$ é a seguinte: primeiramente projetase o espaço de linhas da matriz $A$ ortogonalmente sobre o espaço de linhas conjunto das matrizes $B$ e $C$; posteriormente, decompõe-se o resultado no espaço de linhas da matriz $C$. 


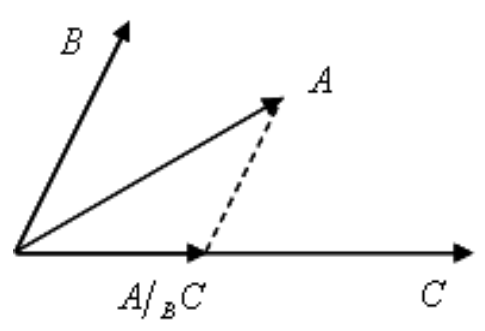

Figura 3.5 - Interpretação de uma projeção obliqua.

Algumas propriedades da projeção obliqua são:

$$
\begin{aligned}
& B /{ }_{B} C=0 \\
& C /{ }_{B} C=C
\end{aligned}
$$

Essas propriedades podem ser usadas para definir projeção obliqua, já que qualquer operação que obedeça (3.37) e (3.38) é considerada uma projeção obliqua.

\subsubsection{Procedimento geral dos algoritmos SID}

Os principais algoritmos de identificação em subespaços que sugiram na década de 90 consideram as seguintes hipóteses (VAN OVERSCHEE; DE MOOR, 1996):

1) O número de dados amostrados e, por conseqüência, o número de colunas das matrizes de blocos de Hankel tendem ao infinito, ou seja, $N, j \rightarrow \infty$.

2) Os ruídos de medição $(v(k))$ e do processo $(w(k))$ são estacionários, possuem média zero e são ruídos brancos.

3) A entrada $u(k)$ é persistentemente excitante de ordem $2 i$ e não é correlacionada com os ruídos não medidos $(v(k)$ e $w(k))$. Devido a essa hipótese, esses algoritmos não são aplicáveis para dados coletados em malha fechada. Como mencionado no início da seção 3.2, existem uma série de novos algoritmos SID desenvolvidos para identificação em malha fechada. Entretanto, eles não serão tratados neste trabalho, pois o controlador SPC, a 
ser estudado a partir do próximo capítulo, utiliza os conceitos dos algoritmos SID tradicionais.

4) O par $\{A, C\}$ é observável, o que implica que todos os modos do sistema podem ser observados na saída $y(k)$. O par $\left\{A,\left(B \quad W^{1 / 2}\right)\right\}$ é controlável, o que implica que todos os modos do sistema são excitados pela entrada externa $u(k)$ ou pelo ruído do processo $w(k)$.

Essas considerações permitem relacionar os dados de entrada e saída do processo de forma conveniente para a determinação das matrizes do sistema.

Os algoritmos SID podem ser divididos em duas principais etapas. Na primeira etapa realiza-se uma projeção das matrizes de blocos de Hankel de entrada e de saída, obtendo-se a matriz de observabilidade estendida $\Gamma_{i}$ ou uma estimativa da seqüência de estados $\tilde{X}_{i}$. A segunda etapa compreende o procedimento de estimação das matrizes do sistema (3.12)-(3.14). A Figura 3.6 mostra de forma esquemática como funcionam os algoritmos SID:

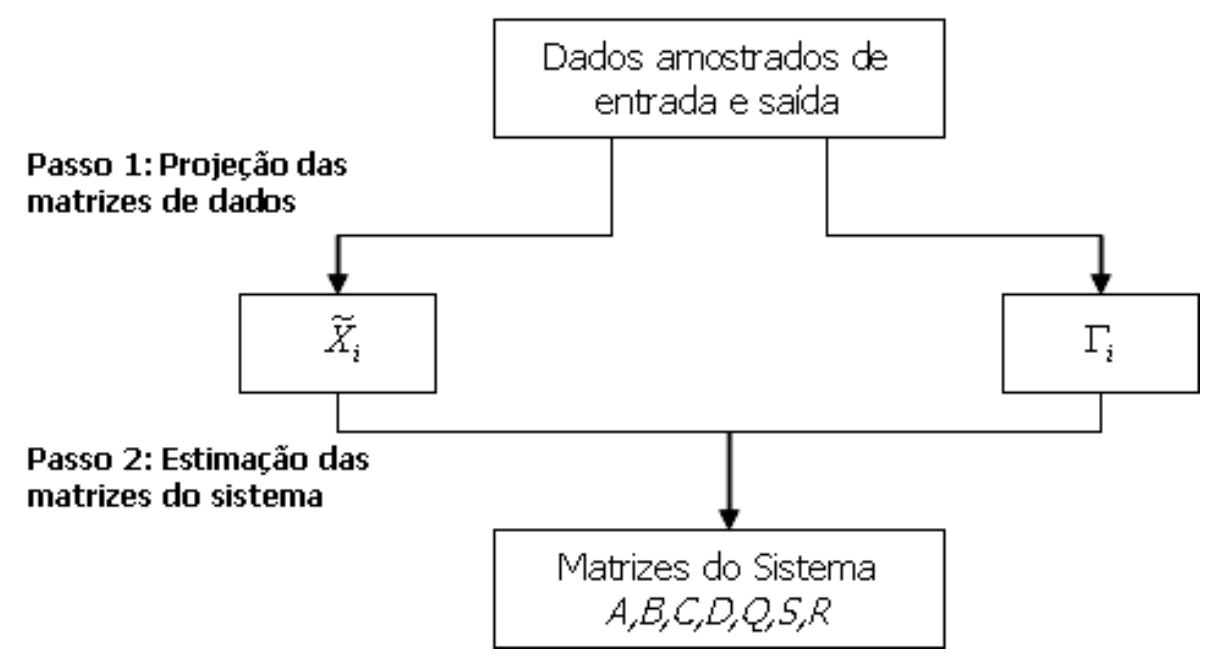

Figura 3.6 - Etapas de um algoritmo de identificação em subespaços.

A projeção realizada na primeira etapa é sobre um subespaço ortogonal ao ruído, de modo que a matriz projetada depende apenas dos componentes determinísticos do sistema. Além disso, antes de serem projetadas, as matrizes de blocos de Hankel de entrada e de saída são ponderadas por outras matrizes, a serem definidas pelo 
algoritmo utilizado. Van Overschee e De Moor (1995) demonstraram que a única diferença entre os algoritmos SID durante a primeira etapa é a escolha dessas matrizes de peso usadas na projeção.

Ainda na primeira etapa, a ordem do modelo deve ser determinada. Isso é feito através de uma decomposição em valores singulares (SVD, do inglês Singular Value Decomposition) da matriz projetada. Essa matriz é de rank deficiente e igual à ordem do modelo $(n x)$. Por isso, na decomposição SVD, apenas $n x$ valores singulares são diferentes de zero.

A segunda etapa, conforme descrito anteriormente, consiste em estimar as matrizes do sistema. Nesse momento, dois tipos de algoritmos podem ser distinguidos: algoritmos que utilizam a seqüência de estados $\left(\tilde{X}_{i}\right)$ para encontrar o modelo e algoritmos que utilizam a matriz de observabilidade estendida $\left(\Gamma_{i}\right)$ nesse procedimento.

Na classe de algoritmos que usam $\tilde{X}_{i}$, as matrizes $A, B, C$ e $D$ são recuperadas em um único passo através da resolução de um problema de minimização de quadrados. Como exemplo desses algoritmos pode-se citar o N4SID (Subspace State Space System IDentification) proposto por Van Overschee e De Moor (1994) e o algoritmo CVA (Canonical Variate Analysis) proposto por Larimore (1990).

A segunda classe de algoritmos utiliza a matriz de observabilidade estendida na determinação das matrizes do sistema. Ao contrário da classe anterior, as matrizes são obtidas em dois passos: primeiramente se obtém as matrizes $A$ e $C$ a partir de $\Gamma_{i}$ e posteriormente as matrizes $B$ e $D$ são computadas. O algoritmo MOESP (Multivariable Output Error State Space), definido em Verhaegen (1994), é um exemplo dessa classe de algoritmos. 


\subsubsection{Algoritmo SID robusto}

A seguir será detalhado o algoritmo robusto de identificação em subespaços proposto em Van Overschee e De Moor (1996). A palavra "robusto" é usada aqui no sentido de que diversas aplicações práticas foram realizadas com sucesso. Esse será o algoritmo utilizado em futuras simulações neste trabalho. Para o estudo de outros métodos de identificação em subespaços recomenda-se a leitura de três livros publicados sobre esse assunto: Van Overschee e De Moor (1996), Huang e Kadali (2008) e Katayama (2005).

O método que será detalhado resolve o problema definido no início desse capítulo: dado um conjunto de dados de entrada e de saída do processo, encontrar a ordem $n x$ do sistema (3.12)-(3.14) e as matrizes $A, B, C, D, W, S$ e $V$. Todas as hipóteses enumeradas anteriormente na seção 3.2.2.3 são consideradas. A idéia aqui é mostrar intuitivamente o procedimento realizado pelo algoritmo. Detalhes matemáticos e demonstrações formais desnecessárias para o entendimento do método não serão apresentados. O leitor interessado em um estudo mais profundo pode consultar Van Overschee e De Moor (1996).

Como mencionado na seção anterior, o primeiro passo de um algoritmo SID é realizar projeções das matrizes de blocos de Hankel de entrada e de saída. 0 algoritmo aqui descrito utiliza duas projeções. A primeira projeção é definida como:

$$
Z_{i} \stackrel{\text { def }}{=} Y_{f} /\left[\begin{array}{l}
W_{p} \\
U_{f}
\end{array}\right]
$$

Pode-se provar que:

$$
Z_{i}=\Gamma_{i} \hat{X}_{i}+H_{i}^{d} U_{f}
$$

As equações (3.39)-(3.40) revelam uma maneira de relacionar a seqüência de estados $\hat{X}_{i}$ com o conjunto de dados amostrados. A matriz $Z_{i}$ é computada a partir dos dados, sem o conhecimento prévio das matrizes do sistema. Essa projeção 
pode ser interpretada como a predição ótima dos dados de saída futuros $Y_{f}$, a partir dos dados de entrada e saída passados $W_{p}$ e as entradas futuras $U_{f}$.

A segunda projeção é definida como:

$$
O_{i} \stackrel{\text { def }}{=} Y_{f} /_{U_{f}} W_{p}
$$

Essa projeção pode então ser ponderada por $\Pi_{U_{f}^{\perp}}$ e a seguinte decomposição em valores singulares é realizada:

$$
O_{i} \Pi_{U_{f}^{\perp}}=\left[\begin{array}{ll}
U_{1} & U_{2}
\end{array}\right]\left[\begin{array}{cc}
S_{1} & 0 \\
0 & 0
\end{array}\right]\left[\begin{array}{l}
V_{1}^{T} \\
V_{2}^{T}
\end{array}\right]=U_{1} S_{1} V_{1}^{T}
$$

A partir das equações (3.41) e (3.42) pode-se demonstrar que:

$$
\begin{aligned}
& O_{i}=\Gamma_{i} \tilde{X}_{i} \\
& \Gamma_{i}=U_{1} S_{1}^{1 / 2}
\end{aligned}
$$

Além disso, demonstra-se também que a ordem $n x$ do sistema (3.12)-(3.14) é igual ao número de valores singulares na equação (3.42) que são diferentes de zero. Algumas observações devem ser feitas em relação à segunda projeção:

1) As equações (3.41)-(3.44) permitem o cálculo do espaço de linhas da seqüência de estados $\tilde{X}_{i}$ e o espaço de colunas da matriz de observabilidade estendida $\Gamma_{i}$ diretamente dos dados amostrados, sem o conhecimento das matrizes do sistema.

2) Deve-se notar que a seqüência de estados $\tilde{X}_{i}$ em (3.43) é diferente da seqüência de estados $\hat{X}_{i}$ em (3.40), no sentido de que cada uma delas utiliza um estado inicial diferente. 
3) Quando o número de dados amostrados não tende ao infinito ou quando o sistema real é não-linear, todos os valores singulares em (3.42) são diferentes de zero. Nesses casos, deve-se estimar a ordem do sistema $(n x)$ como o número de valores singulares "dominantes". Se o modelo identificado obtiver uma ordem elevada é recomendado reduzir a ordem do modelo através de técnicas de redução de ordem (order reduction methods).

Dadas as duas projeções definidas em (3.39) e (3.41), é possível fazer algumas considerações sobre o método de identificação em subespaços. O objetivo da identificação é obter um modelo que preveja da forma mais precisa possível as saídas futuras $\left(Y_{f}\right)$ usando todas as informações que podem ser obtidas do passado $\left(W_{p}\right)$ e com o conhecimento das entradas futuras $\left(U_{f}\right)$. Com base na linearidade do sistema, o método SID propõe combinar o passado $\left(W_{p}\right)$ e as entradas futuras $\left(U_{f}\right)$ linearmente para prever as saídas futuras $\left(Y_{f}\right)$ :

$$
\hat{Y}_{f}=L_{w} W_{p}+L_{u} U_{f}
$$

sendo que $\hat{Y}_{f}$ denota a predição das saídas futuras. A qualidade da predição é então medida pela norma de Frobenius. Matematicamente, a primeira etapa da identificação em subespaços pode ser escrita como:

$$
\min _{L_{w}, L_{u}} \quad\left\|Y_{f}-\left[\begin{array}{ll}
L_{w} & L_{u}
\end{array}\right]\left[\begin{array}{l}
W_{p} \\
U_{f}
\end{array}\right]\right\|_{F}^{2}
$$

A projeção $Z_{i}$ da equação (3.39) é exatamente igual à combinação linear de $W_{p} \mathrm{e}$ $U_{f}:$

$$
Z_{i}=\left[\begin{array}{ll}
L_{w} & L_{u}
\end{array}\right]\left[\begin{array}{l}
W_{p} \\
U_{f}
\end{array}\right]
$$

A combinação ótima do passado para prever o futuro é $L_{w} \cdot W_{p}$, que é exatamente igual à projeção obliqua da equação (3.41): 


$$
O_{i}=L_{w} \cdot W_{p}
$$

Nesse momento, são conhecidas a matriz de observabilidade estendida $\Gamma_{i}$ e a ordem do sistema $n x$. A partir da projeção $Z_{i}$ pode-se definir a seguinte projeção:

$$
Z_{i+1}=Y_{f}^{-} /\left[\begin{array}{l}
W_{p}^{+} \\
U_{f}^{-}
\end{array}\right]=\Gamma_{i-1} \hat{X}_{i+1}+H_{i-1}^{d} U_{f}^{-}
$$

onde os sobrescritos $\bullet^{+} \mathrm{e} \bullet^{-}$nas matrizes de blocos de Hankel correspondem a um descolamento unitário na borda entre passado e futuro, de modo que $U_{p}{ }^{+}=U_{0 l i}$ e $U_{f}{ }^{-}=U_{i+112 i-1}$. Os vetores $Y_{p}^{+}$e $Y_{f}^{-}$são definidos de maneira análoga e a matriz $W_{p}{ }^{+}$é formada pela junção de $Y_{p}^{+}$e $U_{p}{ }^{+}$. Finalmente, a matriz $\Gamma_{i-1}$ é a matriz $\Gamma_{i}$ sem o último bloco de linhas (utiliza-se a notação $\Gamma_{i-1}=\underline{\Gamma_{i}}$ ).

O próximo passo consiste na estimação das matrizes $A, B, C$ e $D$ do sistema. A partir das equações do sistema (3.12)-(3.13), é possível escrever a seguinte relação:

$$
\left[\begin{array}{c}
\hat{X}_{i+1} \\
Y_{i l i}
\end{array}\right]=\left[\begin{array}{cc}
A & B \\
C & D
\end{array}\right]\left[\begin{array}{c}
\hat{X}_{i} \\
U_{i l i}
\end{array}\right]+\left[\begin{array}{c}
\rho_{w} \\
\rho_{v}
\end{array}\right]
$$

sendo:

$$
\begin{aligned}
& \rho_{w}=\left[\begin{array}{llll}
\rho_{w}(i) & \rho_{w}(i+1) & \cdots & \rho_{w}(i+j-1)
\end{array}\right] \\
& \rho_{v}=\left[\begin{array}{llll}
\rho_{v}(i) & \rho_{v}(i+1) & \cdots & \rho_{v}(i+j-1)
\end{array}\right]
\end{aligned}
$$

Os resíduos em cada instante $\rho_{w}(k)$ e $\rho_{v}(k)$ podem ser definidos pelas fórmulas:

$$
\begin{aligned}
& \rho_{w}(k)=\hat{x}(k+1)-\hat{A} \hat{x}(k)-\hat{B} u(k) \\
& \rho_{v}(k)=y(k)-\hat{C} \hat{x}(k)-\hat{D} u(k)
\end{aligned}
$$


Observe que, se as seqüências de estados $\hat{X}_{i}$ e $\hat{X}_{i+1}$ forem conhecidas, então a equação (3.50) pode ser resolvida para as matrizes $A, B, C$ e $D$ através de uma minimização de quadrados. Infelizmente, $\hat{X}_{i}$ e $\hat{X}_{i+1}$ não podem ser calculados diretamente dos dados amostrados. Porém, a partir de (3.40) e (3.49) pode-se escrever:

$$
\begin{aligned}
& \hat{X}_{i}=\Gamma_{i}^{\dagger} \cdot\left(Z_{i}-H_{i}^{d} \cdot U_{f}\right) \\
& \hat{X}_{i+1}=\Gamma_{i-1}^{\dagger} \cdot\left(Z_{i+1}-H_{i-1}^{d} \cdot U_{f}^{-}\right)
\end{aligned}
$$

No lado direito das fórmulas acima, os únicos parâmetros não conhecidos são $H_{i}^{d} \mathrm{e}$ $H_{i-1}^{d}$, visto que $Z_{i}$ e $Z_{i+1}$ podem ser determinadas como projeções das matrizes de blocos de Hankel e os parâmetros $\Gamma_{i}$ e $\Gamma_{i-1}$ são calculados pela equação (3.44). A substituição de (3.53) e (3.54) em (3.50) resulta em:

$$
\left[\frac{\Gamma_{i-1}^{\dagger} \cdot Z_{i+1}}{Y_{i l i}}\right]=\underbrace{\left[\frac{A}{C}\right]}_{\text {termo } 1} \Gamma_{i}^{\dagger} \cdot Z_{i}+\underbrace{\mathrm{K}}_{\text {termo } 2} U_{f}+\underbrace{\left[\frac{\rho_{w}}{\rho_{v}}\right]}_{\text {termo } 3}
$$

sendo

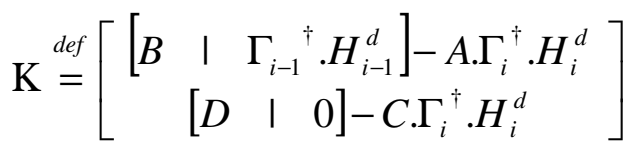

Observe que as matrizes $B$ e $D$ aparecem linearmente na matriz K. A equação (3.55) pode ser resolvida como uma minimização de quadrados para as matrizes $A$, $C$ e K. Dessa maneira obtêm-se duas matrizes do sistema: $A$ e $C$. Com o conhecimento dessas matrizes, deve-se recalcular as matrizes $\Gamma_{i}$ e $\Gamma_{i-1}$ utilizando a equação (3.28). Esse procedimento de recálculo foi adicionado ao algoritmo para aumentar sua robustez.

As matrizes $B$ e $D$ do sistema são então calculadas como:

$$
B, D=\arg \min _{B, D}\left\|\left[\begin{array}{c}
\Gamma_{i-1}^{\dagger} \cdot Z_{i+1} \\
Y_{i l i}
\end{array}\right]-\left[\begin{array}{c}
A \\
C
\end{array}\right] \cdot \Gamma_{i}^{\dagger} \cdot Z_{i}-\mathrm{K}(B, D) \cdot U_{f}\right\|_{F}^{2}
$$


Finalmente as matrizes de covariância $W, S$ e $V$ são determinadas a partir do termo 3 da equação (3.55) como:

$$
\left[\begin{array}{ll}
W & S \\
S^{T} & V
\end{array}\right]=E_{j}\left(\left[\begin{array}{c}
\rho_{w} \\
\rho_{v}
\end{array}\right]\left[\begin{array}{ll}
\rho_{w}{ }^{T} & \rho_{v}^{T}
\end{array}\right]\right)
$$

sendo $E_{j}$ a média sobre um número de dados finito.

O método descrito acima está resumido na Figura 3.7. Ele pode ser implementado de uma maneira numericamente estável e eficiente, através do uso de decomposição QR e SVD. Detalhes da implementação não serão tratados neste trabalho e podem ser vistos de forma bem didática em Van Overschee e De Moor (1996).

\section{Método Robusto de Identificação em Subespaços}

1. Calcular as seguintes projeçồes obliquas:

$$
O_{i}=Y_{f} / \omega_{r} W_{y} \quad Z_{i}=Y_{f} /\left[\begin{array}{l}
W_{y} \\
U_{f}
\end{array}\right] \quad Z_{i+1}=Y_{f}^{-} /\left[\begin{array}{l}
W_{y}^{+} \\
U_{f}^{-}
\end{array}\right]
$$

2. Calcular a decomposiçăo SVD da projeçăo ponderada: $O_{i} \Pi_{U_{r}^{\frac{1}{T}}}=U S V^{T}$

3. Determinar a ordem do sistema pela inspeção dos valores singulares em $S$ e particionar as matrizes da decomposiçấ SVD para se obter $U_{1}$ e $S_{1}$.

4. Determinar $\Gamma_{i}$ e $\Gamma_{i-1}$ como: $\quad \Gamma_{i}=U_{1} S_{1}^{1 / 2}, \quad \Gamma_{i-1}=\underline{\Gamma_{i}}$

5. Resolver o sistema de equações abaimo para $A$ e $C$ :

$$
\left[\frac{\Gamma_{i-1}^{\dagger} \cdot Z_{i+1}}{Y_{\mathrm{di}}}\right]=\left[\frac{A}{C}\right] \Gamma_{i}^{\dagger} \cdot Z_{i}+\mathrm{K} U_{f}+\left[\frac{\rho_{w}}{\rho_{v}}\right]
$$

6. Recalcular $\Gamma_{i}$ e $\Gamma_{i-1}$ a partir de $A$ e $C$.

7. Encontrar $B$ e $D$ como:

$$
B, D=\arg \min _{B, D}\left\|\left[\begin{array}{c}
\Gamma_{i-1}^{\dagger} \cdot Z_{i+1} \\
Y_{\mathrm{j} i}
\end{array}\right]-\left[\begin{array}{c}
A \\
C
\end{array}\right] \Gamma_{i}^{\dagger} \cdot Z_{i}-\mathrm{K}(B, D), U\right\|_{F}^{2}
$$

8. Determinar as matrizes de cowariancia $W, S$ e $V$ de:

$$
\left[\begin{array}{ll}
W & S \\
S^{T} & V
\end{array}\right]=E_{j}\left(\left[\begin{array}{l}
\rho_{w} \\
\rho_{v}
\end{array}\right]\left[\begin{array}{ll}
\rho_{w} & \rho^{T}
\end{array}\right]\right)
$$

Figura 3.7 - Resumo do algoritmo robusto de identificação em subespaços. 


\subsection{APLICAÇÃO DOS MÉTODOS DE IDENTIFICAÇÃO}

Ambos os algoritmos de identificação, PEM e SID, foram aplicados a um sistema real simulado. Primeiramente, na seção 3.3.1, é apresentado o sistema utilizado. Posteriormente, na seção 3.3.2, são mostradas simulações nas quais o sistema é identificado por diferentes métodos. Por fim, uma análise sobre a influência de alguns parâmetros do método SID é realizada na seção 3.3.3.

\subsubsection{Apresentação do sistema da coluna debutanizadora}

O sistema considerado corresponde a uma coluna debutanizadora de uma unidade industrial de destilação da Refinaria RPBC da Petrobras, que foi previamente estudado por Almeida Neto (1999). Na unidade de destilação o petróleo alimenta uma torre de pré-flash, onde é produzida nafta. Essa nafta possui alta volatilidade e por isso é chamada de nafta instável. A coluna debutanizadora, ou estabilizadora, tem por objetivo remover as frações instáveis da nafta. A Figura 3.8 mostra o fluxograma do processo de estabilização da nafta.

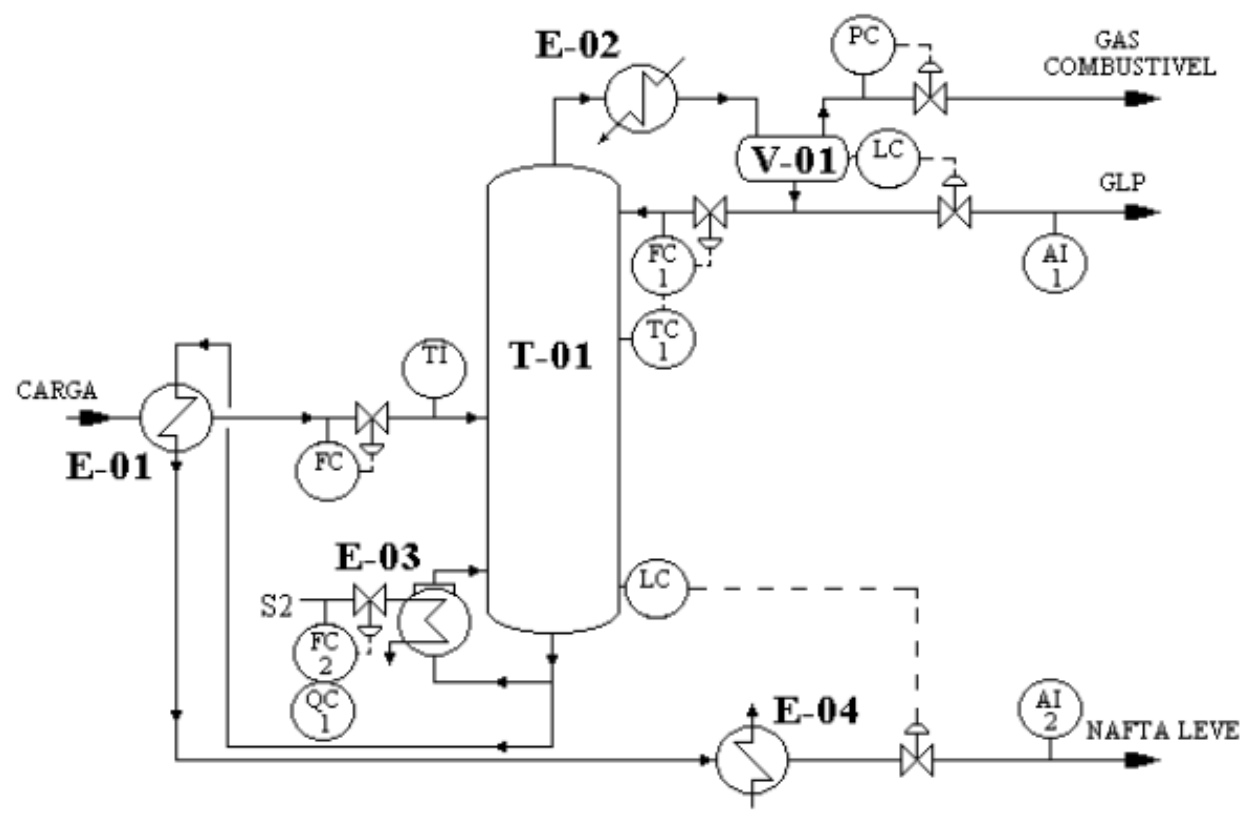

Figura 3.8 - Fluxograma da coluna debutanizadora da refinaria RPBC. 
A carga da coluna debutanizadora, constituída de nafta instável, é proveniente do topo da torre de pré-fracionamento. Ela é pré-aquecida no trocador de calor E-01, que utiliza o produto de fundo da coluna (nafta estabilizada) como líquido de aquecimento, recuperando parte da energia gasta no refervedor E-03. A carga aquecida é então introduzida na torre T-01.

Os vapores de topo da coluna debutanizadora são parcialmente condensados no trocador E-02. O vaso V-01 separa a saída do E-02 em gás combustível e GLP (Gás Liquefeito de Petróleo). O gás combustível é consumido na refinaria, enquanto que o GLP é dividido em duas correntes: uma retorna à coluna como refluxo de topo e a outra é retirada como destilado e segue para estoque. No refervedor E-03, parte do produto de fundo é vaporizado e serve como refluxo para a coluna. A outra parte constitui a nafta estabilizada que vai para o estoque de gasolina.

Para o controle da coluna debutanizadora foram consideradas duas variáveis manipuladas (de entrada) e duas variáveis controladas (de saída), conforme Tabela 3.2 e Tabela 3.3, respectivamente.

Tabela 3.2 - Variáveis manipuladas do sistema da coluna debutanizadora.

\begin{tabular}{|c|c|c|}
\hline Variável & Descrição & Unidade \\
\hline$u_{1}$ & vazão de refluxo de topo & $\mathrm{m}^{3} / \mathrm{d}$ \\
\hline$u_{2}$ & carga térmica do refervedor & MMcal/h \\
\hline
\end{tabular}

Tabela 3.3 - Variáveis controladas do sistema da coluna debutanizadora.

\begin{tabular}{clc}
\hline Variável & \multicolumn{1}{c}{ Descrição } & Unidade \\
\hline$y_{1}$ & $\begin{array}{l}\text { teor de componentes com mais de cinco carbonos } \\
\text { na corrente de GLP no topo }\end{array}$ & $\%$ \\
\hline$y_{2}$ & $\begin{array}{l}\text { pressão de vapor de Reid da gasolina da corrente } \\
\text { de fundo }\end{array}$ & $\mathrm{kPa}$ \\
\hline
\end{tabular}

Em aplicações reais de controle, o modelo do controlador nunca é uma representação perfeita do processo real. Por esse motivo, serão usados dois modelos diferentes para o processo da coluna debutanizadora. Esses modelos, retirados de Ballin (2008), correspondem a duas condições operacionais distintas e são apresentados a seguir: 
$\begin{aligned} \text { Modelo C1: } & G_{1}(s)=\left[\begin{array}{cc}\frac{-0.2623}{60 s^{2}+59.2 s+1} & \frac{0.1368}{1164 s^{2}+99.7 s+1} \\ \frac{0.1242}{218.7 s^{2}+16.2 s+1} & \frac{-0.1351}{70 s^{2}+20 s+1}\end{array}\right] \\ \text { Modelo C2: } & G_{2}(s)=\left[\begin{array}{cc}\frac{-0.2790}{59.77 s^{2}+99.61 s+1} & \frac{0.050}{499.8 s^{2}+73.77 s+1} \\ \frac{0.1950}{220.1 s^{2}+18.93 s+1} & \frac{-0.1722}{29.74 s^{2}+20.71 s+1}\end{array}\right]\end{aligned}$

A unidade de medida do tempo nesses modelos é minuto. Na discretização do modelo utilizou-se um período de amostragem igual a 5 minutos. Para ilustrar a diferença entre esses modelos, a Figura 3.9 apresenta suas respostas ao degrau.
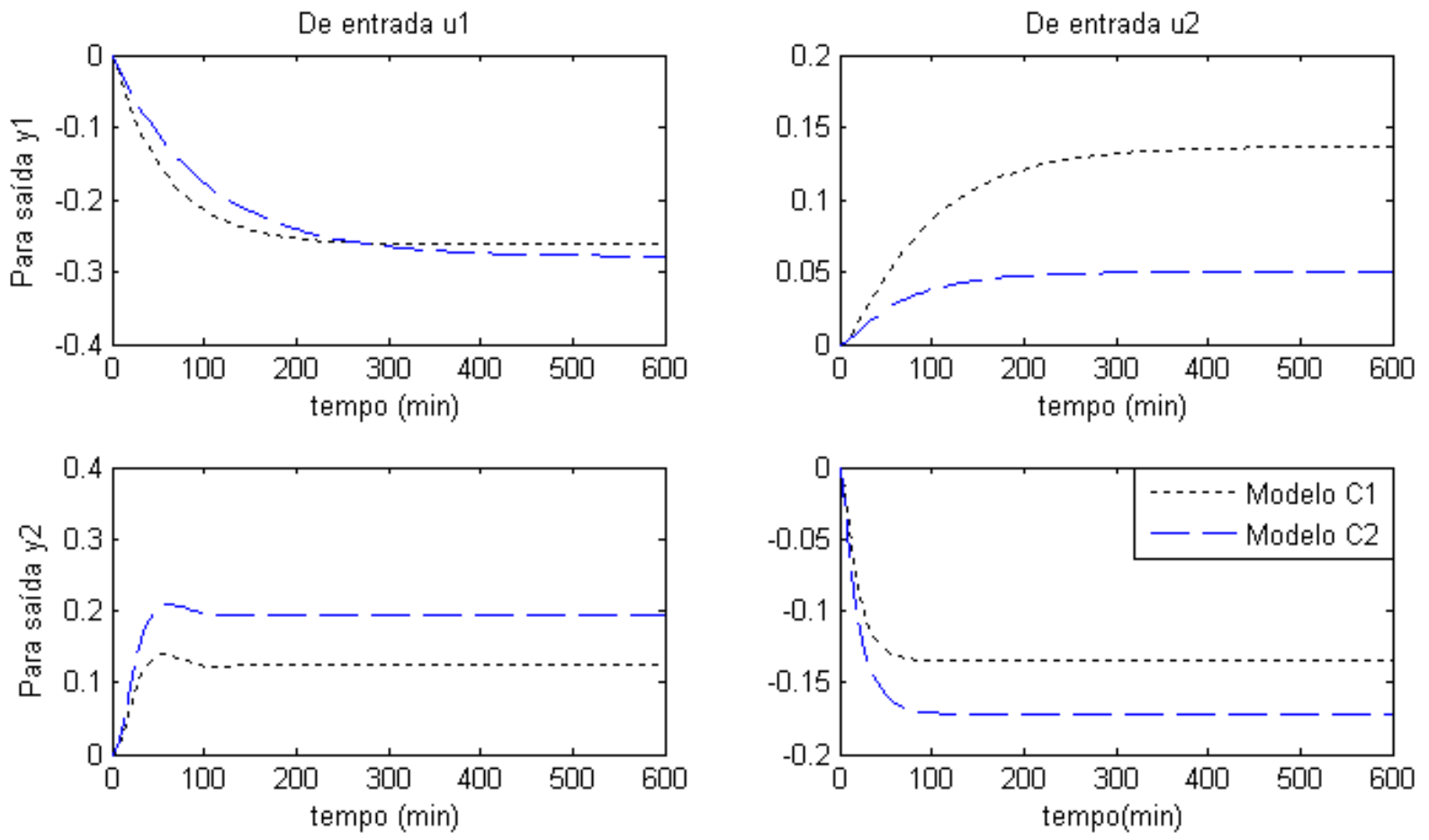

Figura 3.9 - Comparação da resposta ao degrau dos diferentes modelos da coluna debutanizadora.

Analisando a Figura 3.9, percebe-se que os modelos são bem diferentes. Apenas os ganhos da saída $y_{1}$ em relação à entrada $u_{1}$ são similares. Em capítulos posteriores será analisada a influência do erro do modelo em controladores preditivos. 


\subsubsection{Comparações entre os métodos PEM e SID}

Para analisar os métodos de identificação por erro de predição (PEM) e em subespaços (SID), primeiramente realizou-se simulações em Matlab nas quais dados do sistema da coluna debutanizadora foram coletados. Utilizando os dados amostrados, ambos os métodos PEM e SID foram aplicados e os modelos identificados foram comparados.

É importante lembrar que o experimento de coleta de dados deve excitar o sistema de maneira que os dados amostrados tragam informações acerca de todos os modos de interesse do sistema. Em outras palavras, os dados obtidos devem ser persistentemente excitantes. Um tipo de experimento bastante utilizado e que poderia ter sido aplicado neste trabalho é a excitação, em malha aberta, das variáveis de entrada do sistema por um sinal do tipo PRBS (do inglês, Pseudo Random Binary Sequence). Porém, como em capítulos posteriores será estudada a obtenção de dados em malha fechada, optou-se aqui por excitar o sistema dessa maneira. Utilizou-se o método proposto pelo Problema (P3) em Ballin (2008), que corresponde a um controlador MPC com excitação interna. Detalhes desse controlador não serão tratados. Deve-se apenas ter em mente que foram realizados experimentos em malha fechada para a coleta de dados.

Como os dados amostrados são medidos diretamente da planta, o erro do modelo do controlador MPC com excitação interna não influencia a identificação. Dessa maneira, por questões de simplicidade, considerou-se que o modelo da planta e do controlador são iguais e representados pelo Modelo C1. Os valores de referências (set-points) das variáveis de saída foram mantidos na origem, isto é, $y^{s p}=\left[\begin{array}{ll}0 & 0\end{array}\right]^{T}$. Diferentes experimentos foram realizados para se coletar dados nas condições de ausência e presença de ruído. Para o caso com ruído, considerou-se ruído randômico adicionado diretamente nas saídas do processo. Diferentes simulações foram realizadas para diferentes intensidades do ruído, que foi controlada pelo valor da variância. Foram amostrados 1500 pontos em cada teste. A Tabela 3.4 resume os experimentos realizados. 
Tabela 3.4 - Descrição dos experimentos de coleta de dados para a coluna debutanizadora.

\begin{tabular}{cc}
\hline Teste & Descrição \\
\hline A & Caso sem ruído na planta \\
\hline B & Caso com ruído adicionado à saída com variância de 0.0001 \\
\hline C & Caso com ruído adicionado à saída com variância de 0.0005 \\
\hline D & Caso com ruído adicionado à saída com variância de 0.001 \\
\hline E & Caso com ruído adicionado à saída com variância de 0.01 \\
\hline
\end{tabular}

As Figura 3.10 e Figura 3.11 apresentam os dados obtidos pelos experimentos $A$ e $E$, respectivamente. Observa-se que a excitação inserida no sistema causou oscilações das variáveis controladas ao redor de seus set-points. Pode-se notar também que, no caso com ruído (Figura 3.11), as saídas possuem oscilações maiores do que aquelas geradas no caso sem ruído (Figura 3.10), apesar das intensidades das oscilações das entradas serem iguais em ambos os casos. Isso ocorre exatamente devido à adição do distúrbio na saída.
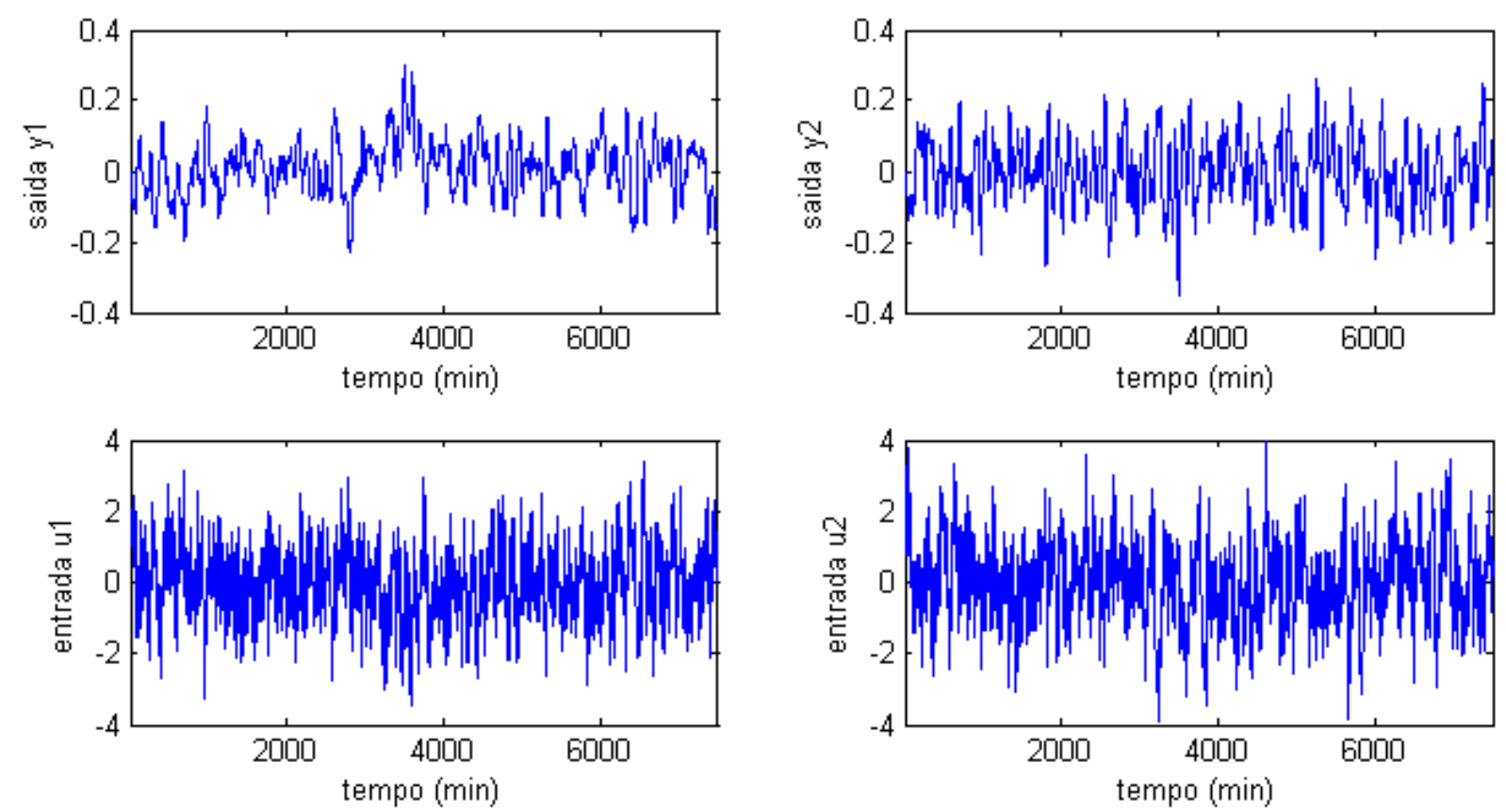

Figura 3.10 - Dados amostrados pelo experimento de identificação $A$. Planta sem ruído. 

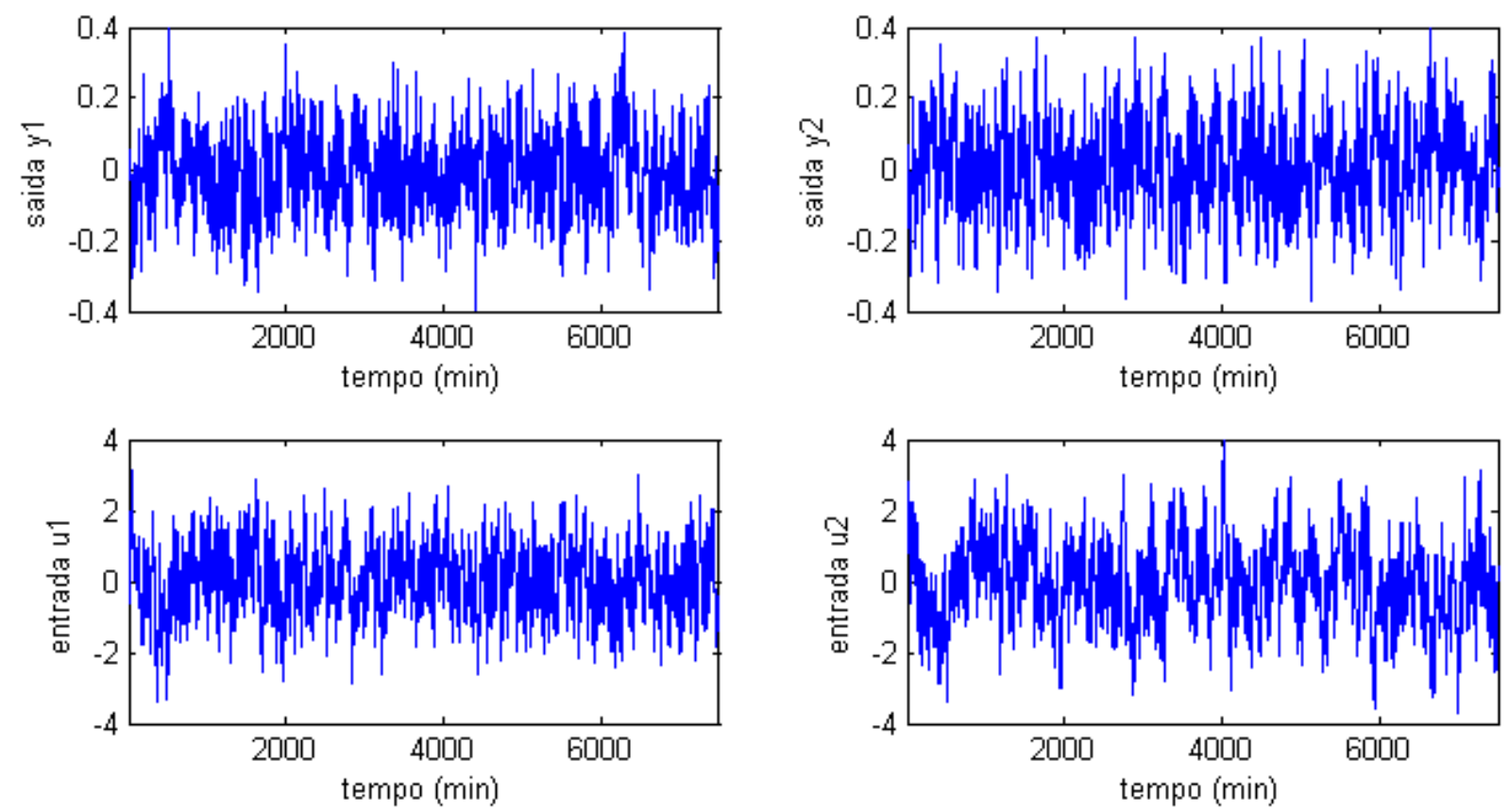

Figura 3.11 - Dados amostrados pelo experimento de identificação $E$. Ruído aleatório de variância 0.01 adicionado à saída da planta.

Com os dados coletados, o próximo passo consiste em aplicar os métodos de identificação. Para o método PEM foi considerada uma estrutura de modelo ARX, que é a estrutura real do sistema (Modelo C1). O critério utilizado foi a minimização dos quadrados dos erros de predição, conforme equação (3.8). Em uma identificação real, deve-se encontrar a ordem dos polinômios do modelo, os parâmetros $n_{a}$ e $n_{b}$ da equação (3.4), de forma iterativa. Porém, como nesse caso simulado a ordem do sistema real é conhecida, assumiu-se $n_{a}=4$ e $n_{b}=4$ para cada saída do sistema. Apesar dos polinômios da função de transferência do Modelo C1 serem de segunda ordem, a estrutura ARX considera um denominador único para as entradas com relação a cada saída, o que aumenta a ordem do sistema para quatro.

O método SID considera o modelo em espaço de estados e o único parâmetro a ser escolhido previamente é o número de blocos de linhas $i$ utilizado nas matrizes de blocos de Hankel de entrada e de saída. Esse parâmetro tem como pré-requisito ser no mínimo igual à ordem $n x$ do modelo identificado. Além disso, o número de colunas $j$ nas matrizes de Hankel (que está diretamente relacionado ao número de dados usados na identificação) deve ser muito maior que o número de linhas ( $i$ ). Tipicamente $j$ é 100 vezes maior que $i$ (FAVOREEL; DE MOOR, 1998). Para esta 
simulação, foi escolhido $i=15$. A ordem do modelo em espaço de estados é determinada durante o procedimento de identificação, porém sabe-se que, para o sistema real, $n x=8$, visto que um sistema de duas entradas e duas saídas com funções de transferência de segunda ordem gera um modelo com 8 estados.

Após a aplicação dos algoritmos de identificação, foi realizada validação cruzada para verificar o desempenho dos modelos obtidos. Dos 1500 dados amostrados em cada teste, os primeiros 1000 dados foram usados na identificação e os 500 dados finais foram guardados para validação. Na etapa de validação, os dados reais foram comparados com as respostas preditas pelos modelos e o índice FIT foi calculado. Esses procedimentos foram realizados através do comando compare do Matlab. Além disso, as respostas ao degrau dos modelos identificados foram comparadas com a resposta ao degrau do sistema real (Modelo C1).

O primeiro procedimento de identificação foi realizado com os dados obtidos no experimento $A$. O resultado da validação cruzada do modelo obtido pelo método PEM está mostrado na Figura 3.12.
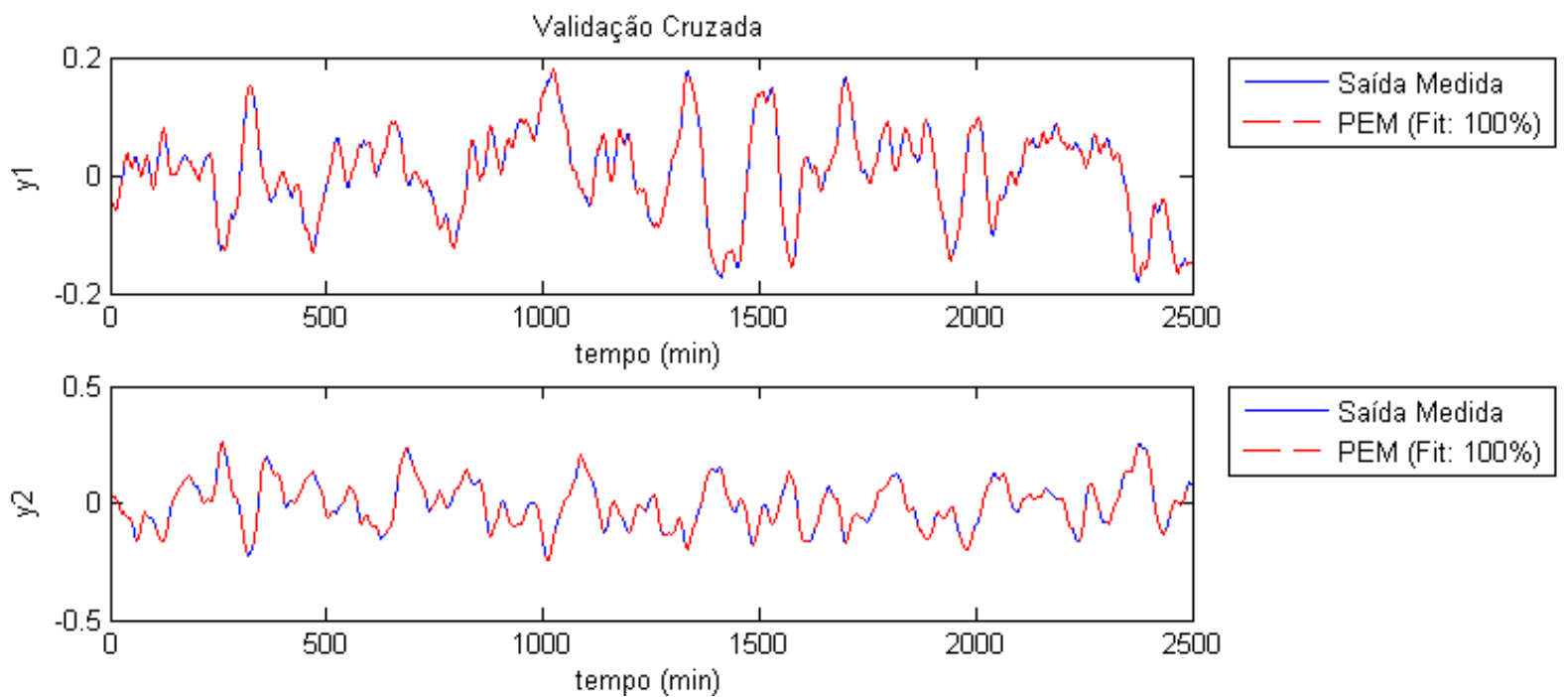

Figura 3.12 - Validação cruzada do modelo identificado pelo método PEM para os dados coletados no experimento $A$. Planta sem ruído.

No método SID é necessário determinar a ordem do modelo através de uma inspeção nos valores singulares da decomposição SVD mostrada na equação (3.42). A Figura 3.13 mostra, em ordem decrescente, os valores singulares obtidos nessa decomposição. 


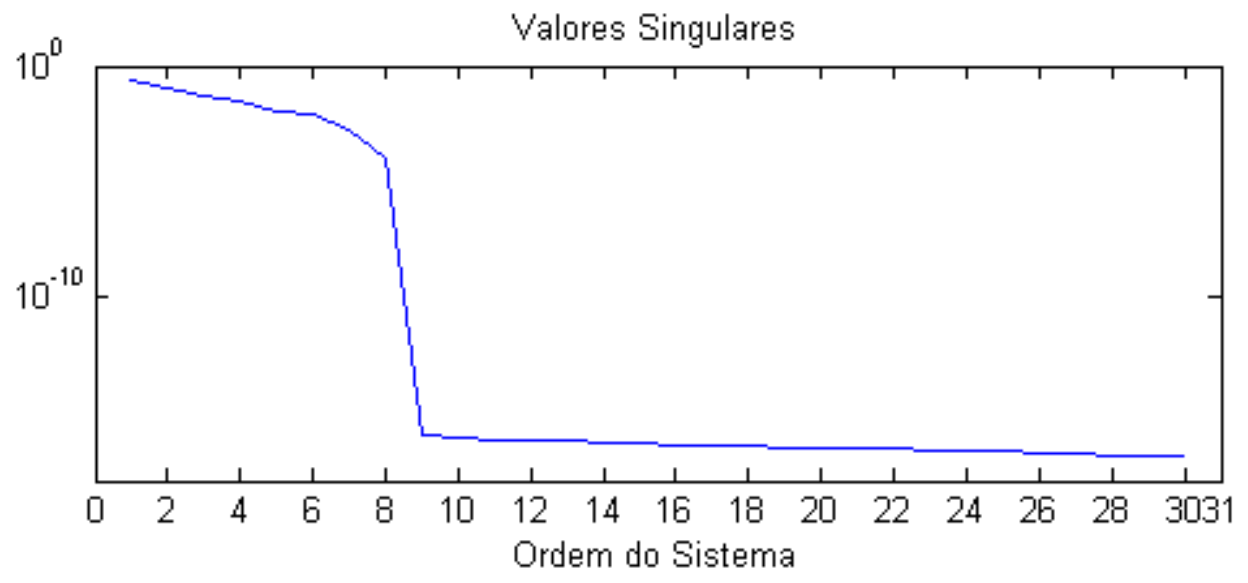

Figura 3.13 - Determinação da ordem do modelo no método SID para os dados do experimento $A$. Planta sem ruído.

Pode-se observar na Figura 3.13 que o nono valor singular é muito menor que os oito primeiros. A partir desse ponto, os valores singulares permanecem muito baixos, na ordem de $10^{-15}$. Portanto, fica bem claro que a ordem do modelo a ser escolhida deve ser 8, que, como mencionado anteriormente, é a ordem real do sistema. A Figura 3.14 mostra o resultado da validação cruzada para o modelo obtido pelo método SID.
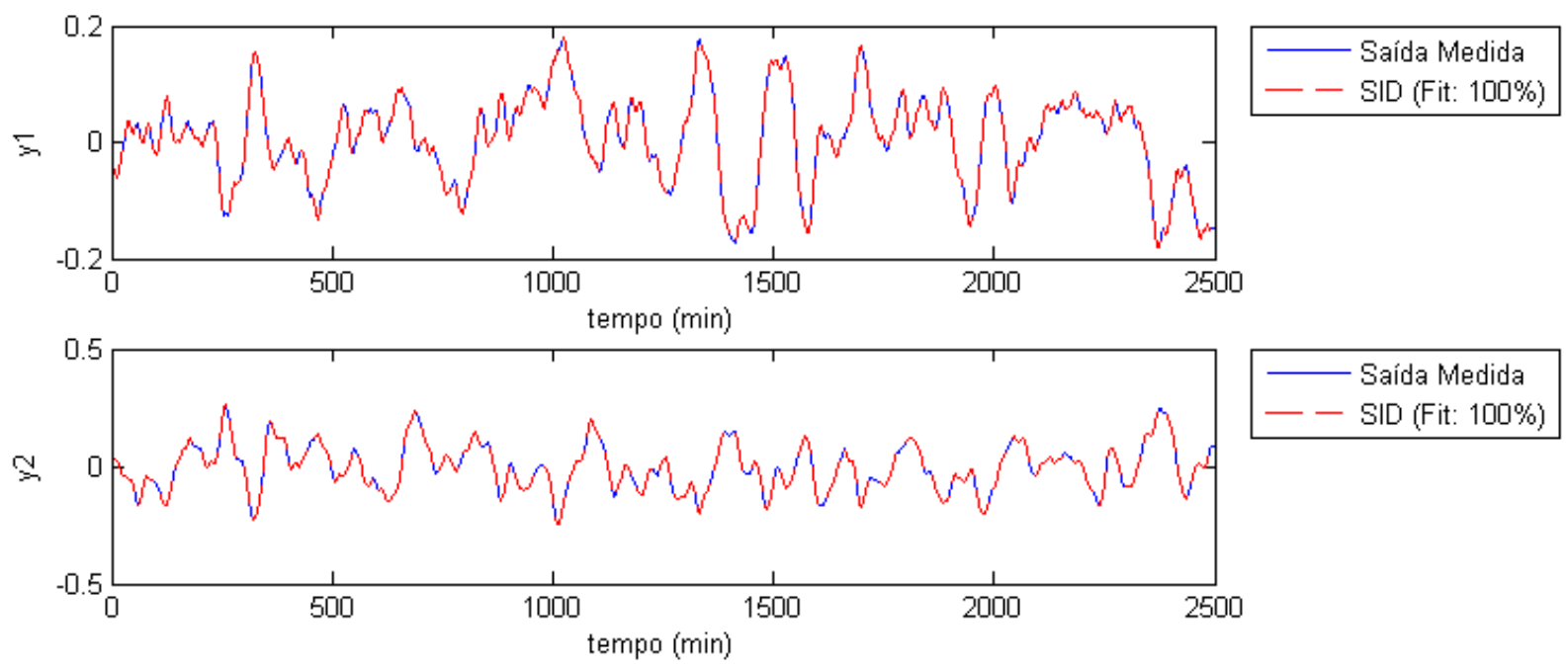

Figura 3.14 - Validação cruzada do modelo identificado pelo método SID para os dados coletados no experimento $A$. Planta sem ruído.

Finalmente a Figura 3.15 ilustra a resposta ao degrau do sistema real e dos modelos obtidos por ambos os métodos de identificação: 

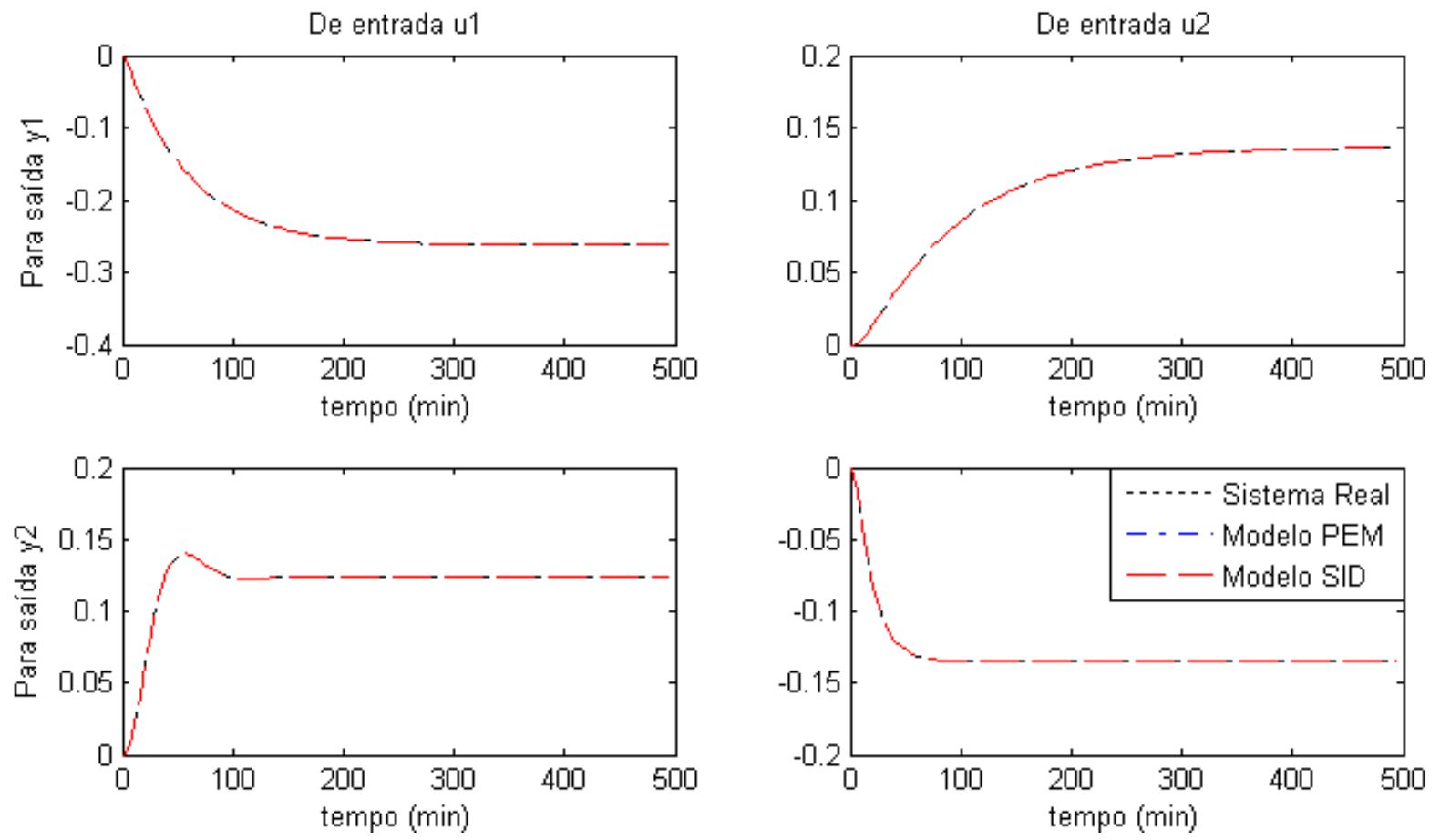

Figura 3.15 - Comparação das respostas ao degrau do sistema real e dos modelos obtidos via PEM e SID para os dados do experimento $A$. Planta sem ruído.

Analisando as Figura 3.12, Figura 3.13 e Figura 3.15, percebe-se que ambos os métodos de identificação obtiveram modelos perfeitos. O FIT calculado foi de $100 \%$ para os dois modelos e suas respostas ao degrau foram idênticas à resposta ao degrau do sistema real. Esse comportamento era esperado, pois os dados amostrados não continham nenhum ruído e o sistema é, de fato, linear e invariante no tempo.

As Figura 3.16, Figura 3.17, Figura 3.18 e Figura 3.19 apresentam os resultados obtidos na identificação com os dados coletados no experimento $C$ (caso com ruído randômico de variância 0.0005 adicionado à saída).

Analisando as Figura 3.16, Figura 3.18 e Figura 3.19 observa-se que a adição de ruído no experimento de coleta de dados piorou a qualidade dos modelos identificados por ambos os métodos. O modelo obtido via PEM teve um desempenho pior que o modelo em espaço de estados identificado via SID. Esse fato pode ser verificado tanto pelos valores de FIT calculados como pelas respostas ao degrau. Na Figura 3.19, observa-se que, especialmente para a variável $y_{1}$, a resposta do modelo obtido via PEM ficou bem distante da resposta do sistema real. 
A resposta ao degrau do modelo obtido pelo método SID ficou muito próxima da resposta da planta. Porém, os valores de FIT calculados para esse modelo ficaram na faixa de $75-80 \%$, que são valores não tão elevados. A explicação para esse fato é que os dados utilizados na validação apresentam ruídos que não são previstos no modelo, afetando negativamente o índice FIT. Por outro lado, na comparação das respostas ao degrau, a resposta do sistema real está livre de qualquer distúrbio.
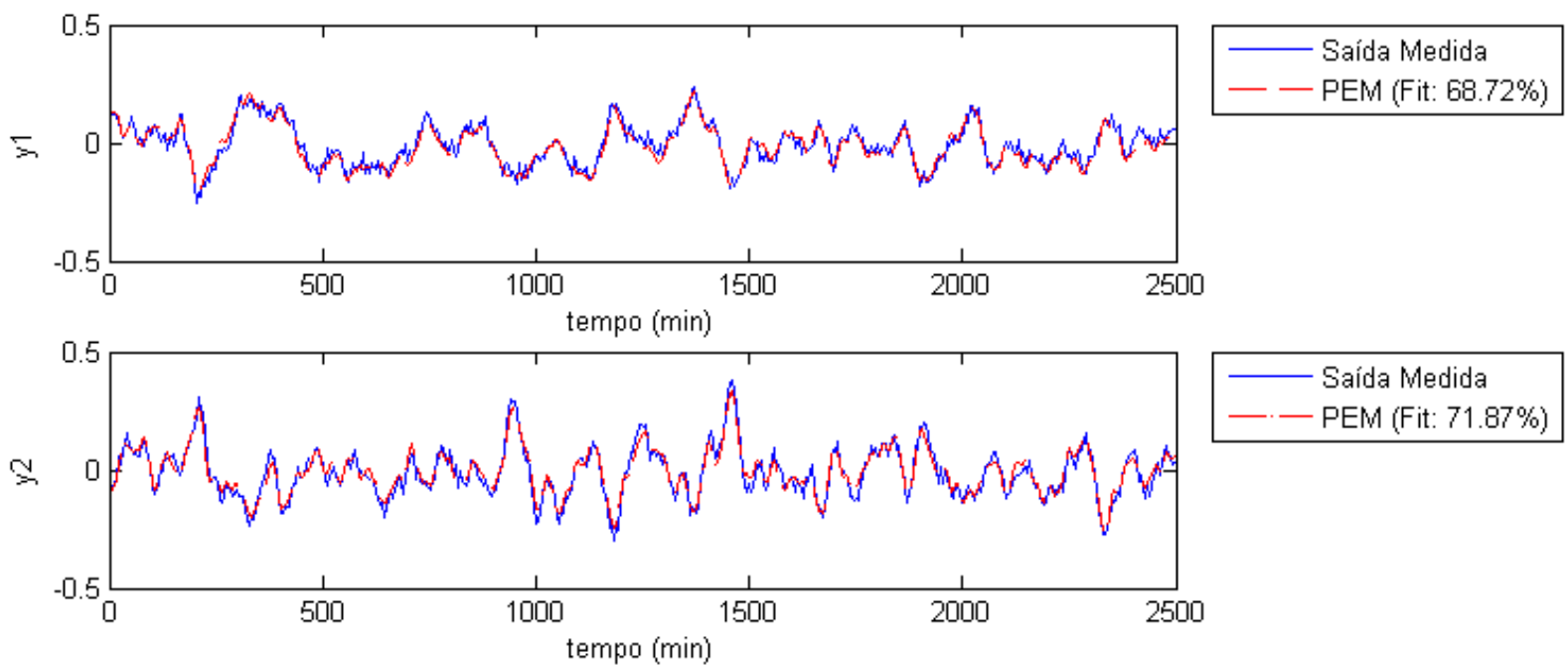

Figura 3.16 - Validação cruzada do modelo identificado pelo método PEM para os dados coletados no experimento $C$. Ruído de variância 0.0005 adicionado à saída da planta.

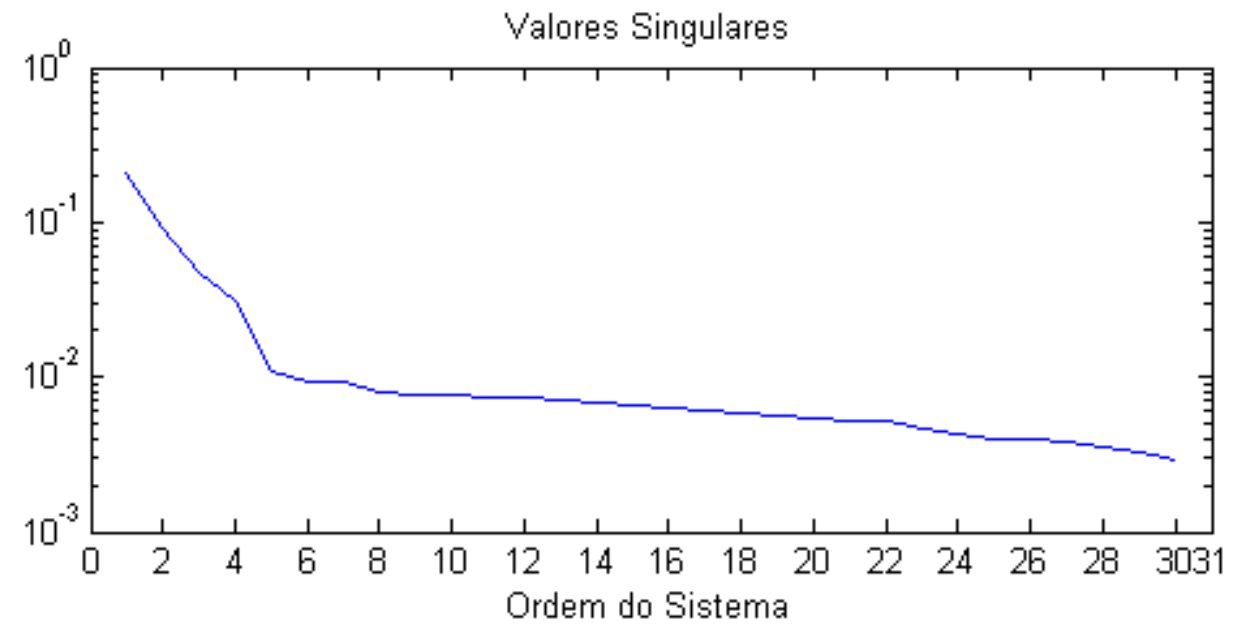

Figura 3.17- Determinação da ordem do modelo no método SID para os dados do experimento $C$. Ruído de variância 0.0005 adicionado à saída. 

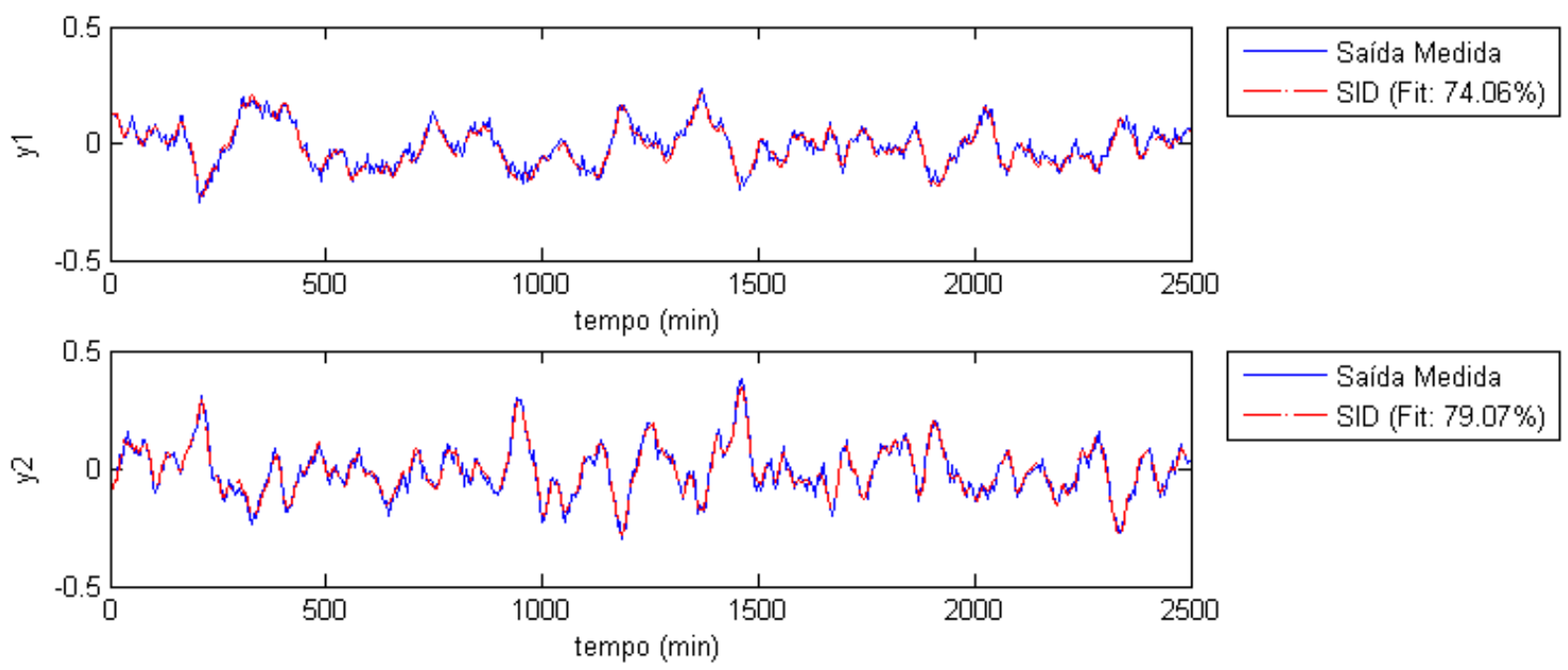

Saída Medida

—- — SID (Fit: 79.07\%)

Figura 3.18 - Validação cruzada do modelo identificado pelo método SID para os dados coletados no experimento $C$. Ruído de variância 0.0005 adicionado à saída da planta.
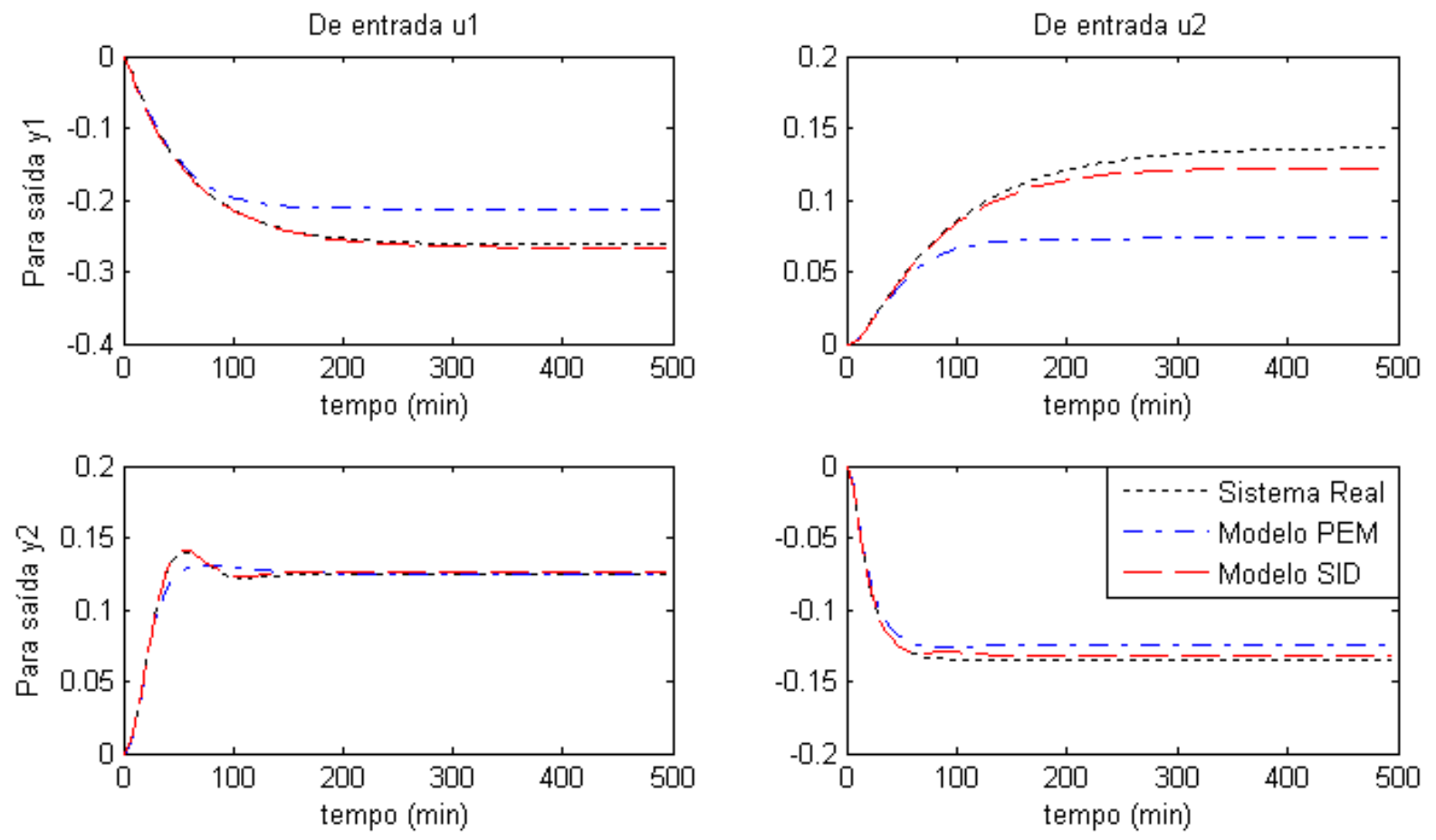

Figura 3.19 - Comparação das respostas ao degrau do sistema real e dos modelos obtidos via PEM e SID para os dados do experimento $C$. Ruído de variância 0.0005 adicionado à saída da planta.

Apesar do resultado satisfatório obtido pela identificação em subespaços, uma desvantagem dessa técnica é a dificuldade de se determinar a ordem do modelo quando há ruídos correlacionados com a entrada ou quando o sistema é não-linear. No teste realizado, o sistema é linear, mas como a excitação dos dados foi feita em malha fechada, o ruído é correlacionado com a entrada. Analisando a Figura 3.17, observa-se que não é claro o momento em que os valores singulares podem passar 
a ser considerados como zero. Como se sabia a ordem do sistema, escolheu-se para o modelo a ordem correta, porém isso não seria possível em um caso real.

O procedimento de identificação foi repetido para cada conjunto de dados. A Tabela 3.5 resume os resultados obtidos.

Tabela 3.5 - Resumo dos testes de identificação e validação feitos para a coluna debutanizadora.

\begin{tabular}{|c|c|c|c|c|c|c|c|}
\hline \multirow[t]{2}{*}{ Teste } & \multirow{2}{*}{$\begin{array}{l}\text { Variância do } \\
\text { Ruído }\end{array}$} & \multicolumn{3}{|c|}{ FIT Modelo PEM } & \multicolumn{3}{|c|}{ FIT Modelo SID } \\
\hline & & y1 & y2 & Médio & y1 & y2 & Médio \\
\hline$A$ & 0 & $100.0 \%$ & $100.0 \%$ & $100.0 \%$ & $100.0 \%$ & $100.0 \%$ & $100.0 \%$ \\
\hline$B$ & 0.0001 & $79.3 \%$ & $81.8 \%$ & $80.6 \%$ & $85.8 \%$ & $88.2 \%$ & $87.0 \%$ \\
\hline C & 0.0005 & $68.7 \%$ & $71.9 \%$ & $70.3 \%$ & $74.1 \%$ & $79.1 \%$ & $76.6 \%$ \\
\hline $\mathrm{D}$ & 0.001 & $60.5 \%$ & $62.5 \%$ & $61.5 \%$ & $62.4 \%$ & $67.7 \%$ & $65.1 \%$ \\
\hline $\mathrm{E}$ & 0.01 & $15.8 \%$ & $25.5 \%$ & $20.7 \%$ & $21.5 \%$ & $27.2 \%$ & $24.4 \%$ \\
\hline
\end{tabular}

Em todos os testes, nota-se que a identificação em subespaços resultou em um valor de FIT maior do que a identificação pelo método do erro de predição, porém essa diferença é pequena. O método SID mostrou-se ser uma boa alternativa para o método clássico PEM, mesmo quando os dados são coletados em malha fechada.

Observa-se que a qualidade dos modelos identificados em ambos os métodos decai conforme aumenta a intensidade do distúrbio. Para distúrbios com variância de 0.01, os modelos identificados são muito ruins, obtendo valores de FIT entre $20 \%$ e $30 \%$. Esse fato ilustra a importância da intensidade do ruído ser muito menor que a intensidade da excitação. Outra alternativa é realizar um pré-tratamento dos dados, utilizando um filtro que remova ruídos de alta freqüência.

\subsubsection{Análise detalhada do método SID}

A identificação em subespaços possui, em teoria, um único parâmetro que deve ser previamente definido: o número de linhas das matrizes de blocos de Hankel. Através da inspeção dos valores singulares da decomposição SVD de uma matriz projetada, determina-se a ordem do modelo em espaço de estados. Porém, como visto na seção anterior, quando há ruídos correlacionados com a entrada ou o sistema é não- 
linear, essa determinação da ordem do modelo não é óbvia. Portanto, na prática, pode-se considerar a ordem do modelo como um segundo parâmetro a ser informado na identificação.

Diversas simulações foram realizadas para estudar a influência do número de linhas $i$ das matrizes de blocos de Hankel e da ordem $n x$ do modelo. Em todos os testes foram utilizados os dados coletados pelo experimento $B$, que possui ruído randômico de variância 0.0001 adicionado à saída. Considerou-se como caso base a identificação com $i=15$ e $n x=8$. Para esse caso, as Figura 3.20, Figura 3.21 e Figura 3.22 mostram os resultados da identificação pelo método SID. Os resultados mostrados são os mesmos resumidos na Tabela 3.5 para o experimento $B$.

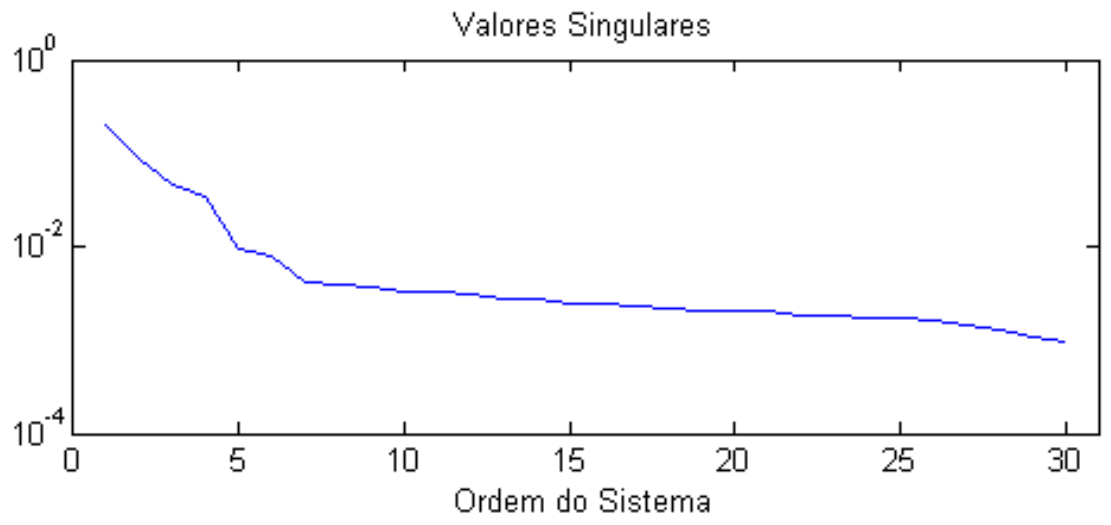

Figura 3.20 - Determinação da ordem do modelo no método SID para os dados do experimento $B$. Ruído de variância 0.0001 adicionado à saída da planta.
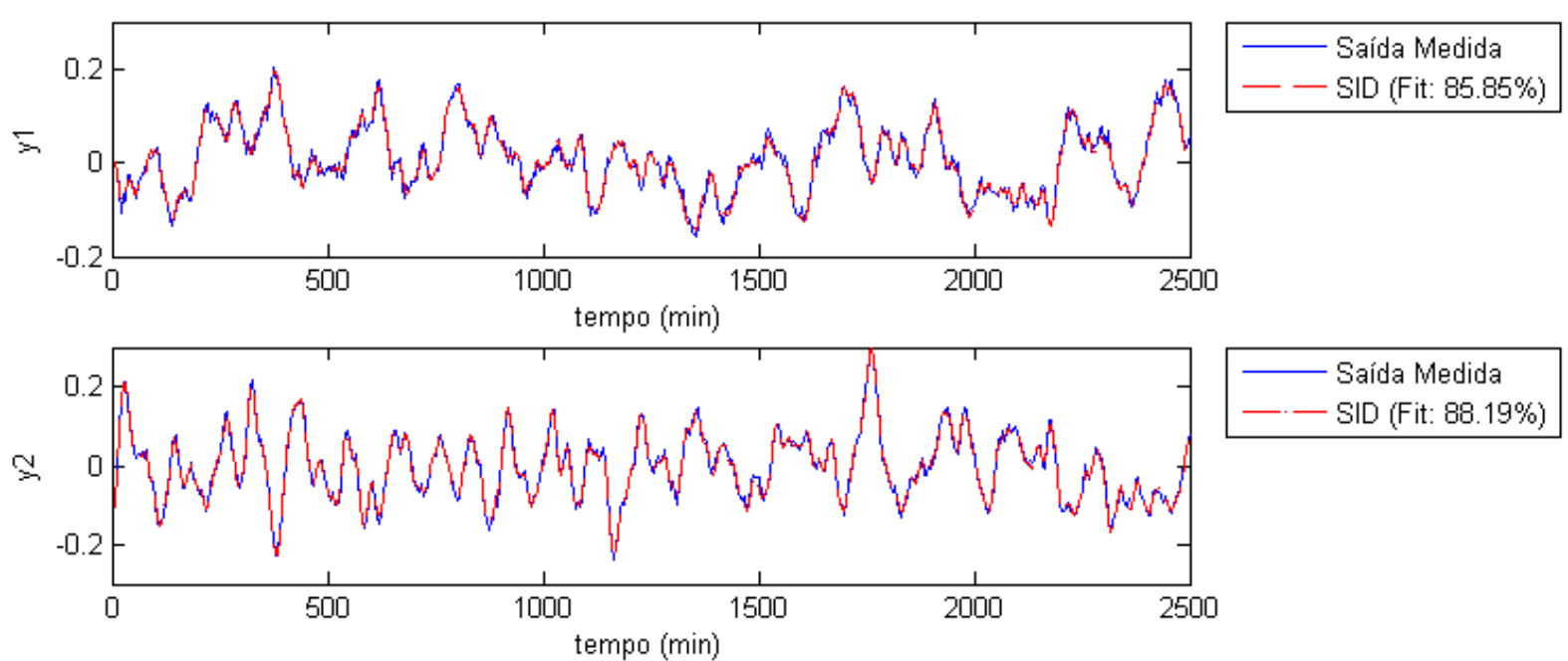

Figura 3.21 - Validação cruzada do modelo identificado pelo método SID para os dados coletados no experimento $B$. Ruído de variância 0.0001 adicionado à saída da planta. 

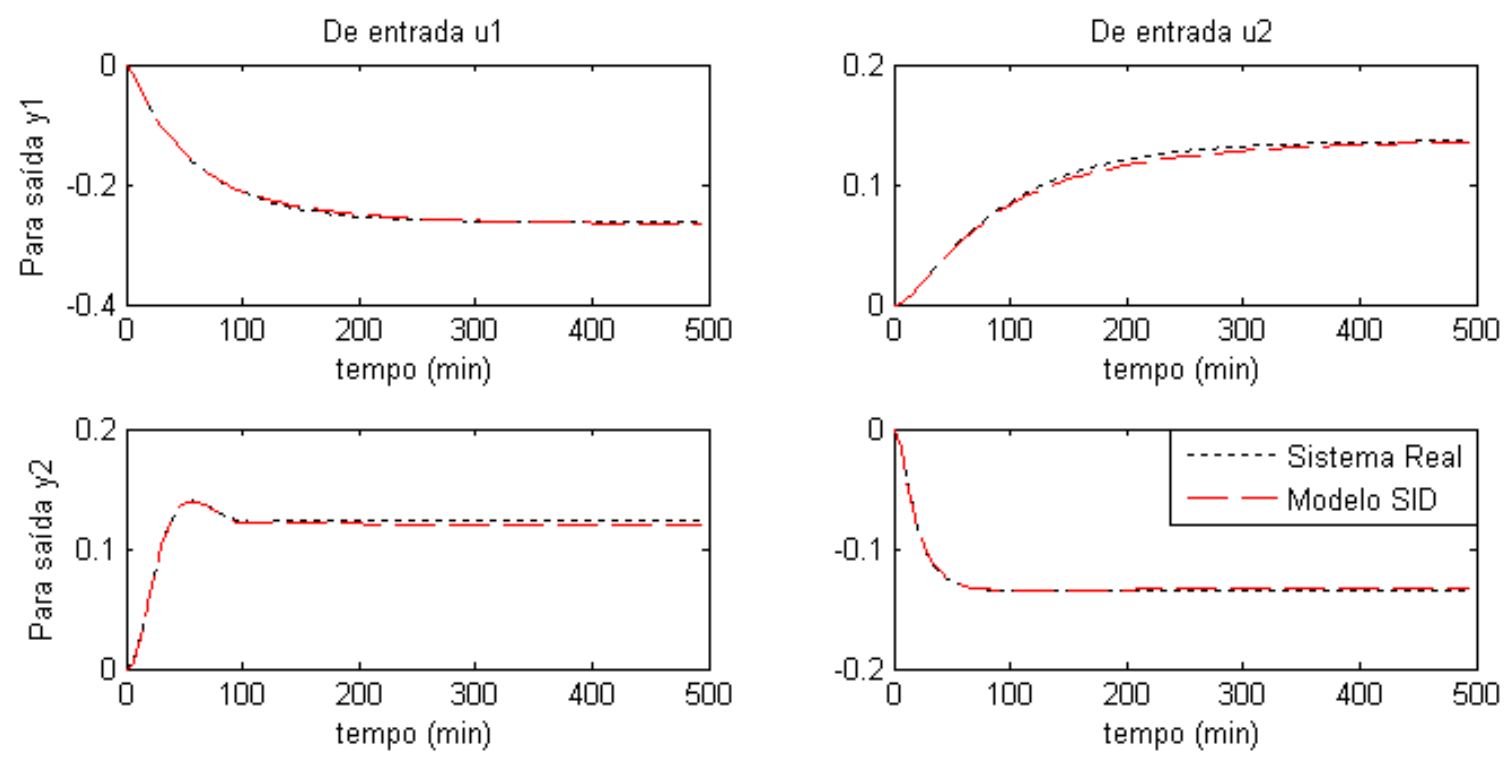

Figura 3.22 - Comparação das respostas ao degrau do sistema real e do modelo obtido via SID para os dados do experimento $B$. Ruído de variância 0.0001 adicionado à saída da planta.

Com base nessa identificação, foram feitos diferentes testes mantendo-se a ordem do modelo constante e alterando-se o número de linhas das matrizes de Hankel, conforme Tabela 3.6. Posteriormente foi realizado o procedimento contrário, mostrado na Tabela 3.7.

\begin{tabular}{ccccc}
\multicolumn{5}{c}{ Tabela 3.6 - Resumo dos testes para análise de influência do parâmetro $i$ no método SID. } \\
\hline Teste & $\begin{array}{c}\text { Blocos de linhas das } \\
\text { matrizes de Hankel }\end{array}$ & y1 & Índice FIT \\
& 7 & $82.6 \%$ & $88.0 \%$ & Médio \\
\hline $1 \mathrm{~A}$ & 8 & $78.6 \%$ & $85.8 \%$ & $82.3 \%$ \\
\hline $1 \mathrm{~B}$ & 9 & $83.4 \%$ & $88.1 \%$ & $85.8 \%$ \\
\hline $1 \mathrm{C}$ & 10 & $84.5 \%$ & $88.1 \%$ & $86.3 \%$ \\
\hline $1 \mathrm{D}$ & 12 & $85.3 \%$ & $88.3 \%$ & $86.8 \%$ \\
\hline $1 \mathrm{E}$ & 15 & $85.8 \%$ & $88.2 \%$ & $87.0 \%$ \\
\hline $1 \mathrm{~F}$ & 20 & $85.9 \%$ & $88.1 \%$ & $87.0 \%$ \\
\hline $1 \mathrm{G}$ & 30 & $85.8 \%$ & $88.3 \%$ & $87.1 \%$ \\
\hline $1 \mathrm{H}$ & 50 & $85.9 \%$ & $88.3 \%$ & $87.1 \%$ \\
\hline $1 \mathrm{I}$ & 100 & $85.4 \%$ & $87.4 \%$ & $86.4 \%$ \\
\hline $1 \mathrm{~J}$ & & & &
\end{tabular}

Obs: ordem do modelo $=8$ (cte)

Analisando a Tabela 3.6, percebe-se que, com exceção dos testes $1 B$ e $1 \mathrm{~J}$, conforme se aumenta o número de linhas $i$, há uma melhora no modelo identificado. O teste $1 \mathrm{~J}$ mostra que se $i$ for muito elevado, o desempenho da identificação começa a decair. Uma explicação para esse fato é que a hipótese de o número de colunas $j$ tender ao infinito, ou seja, ser muito maior que o número de linhas, passa a não ser verdadeira, uma vez que o número de dados amostrados permanece o 
mesmo. Pode-se notar que a partir de $i=15$, praticamente não há diferença no fator FIT calculado na validação. O pior valor de FIT ocorreu para $i=8$, caso em que o número de linhas coincide com a ordem real do sistema.

Tabela 3.7 - Resumo dos testes para análise de influência do parâmetro $n x$ no método SID.

\begin{tabular}{ccccc}
\hline \multirow{2}{*}{ Teste } & \multirow{2}{*}{ Ordem do Modelo } & y1 & $\begin{array}{c}\text { Índice FIT } \\
\text { y2 }\end{array}$ & Médio \\
\hline $2 \mathrm{~A}$ & 5 & $83.8 \%$ & $84.4 \%$ & $84.1 \%$ \\
\hline $2 \mathrm{~B}$ & 6 & $85.8 \%$ & $88.4 \%$ & $87.1 \%$ \\
\hline $2 \mathrm{C}$ & 7 & $85.7 \%$ & $88.2 \%$ & $87.0 \%$ \\
\hline $2 \mathrm{D}$ & 8 & $85.8 \%$ & $88.2 \%$ & $87.0 \%$ \\
\hline $2 \mathrm{E}$ & 9 & $85.5 \%$ & $88.3 \%$ & $86.9 \%$ \\
\hline $2 \mathrm{~F}$ & 10 & $85.5 \%$ & $88.4 \%$ & $87.0 \%$ \\
\hline $2 \mathrm{G}$ & 11 & $85.8 \%$ & $88.4 \%$ & $87.1 \%$ \\
\hline $2 \mathrm{H}$ & 12 & $85.8 \%$ & $88.4 \%$ & $87.1 \%$ \\
\hline $2 \mathrm{I}$ & 15 & $85.7 \%$ & $88.4 \%$ & $87.1 \%$ \\
\hline $2 \mathrm{~J}$ & 20 & $85.9 \%$ & $88.1 \%$ & $87.0 \%$ \\
\hline $2 \mathrm{~K}$ & 25 & $71.6 \%$ & $84.6 \%$ & $78.1 \%$ \\
\hline
\end{tabular}

Obs: número de blocos de linhas das matrizes de Hankel = 15 (cte)

$\mathrm{Na}$ análise da influência da ordem do modelo (Tabela 3.7), percebe-se que o desempenho do modelo identificado ficou praticamente igual no intervalo $6 \leq n x \leq 20$. $\mathrm{O}$ teste $2 \mathrm{~K}$ demonstrou que a escolha de uma ordem muito elevada pode deteriorar a qualidade do modelo obtido. Verificou-se que o SID apresentou resultados satisfatórios para uma faixa larga de valores da ordem do modelo. Para $n x>8,0$ modelo identificado não é mínimo e pode passar por um procedimento de redução de ordem. Porém, não era esperado que modelos com $n x<8$, que corresponde à ordem mínima do sistema, obtivessem um bom resultado, pois nesses casos o modelo obtido não possui estados suficientes para a determinação completa do comportamento do sistema. Entretanto, o sistema em questão possui pólos muito próximos de zero, que são desprezíveis em relação aos outros pólos. Assim, um modelo que considera somente os pólos dominantes pode resultar em uma boa representação do sistema. 


\section{CONTROLE PREDITIVO COM ENFOQUE EM SUBESPAÇOS}

Controle preditivo baseado em modelo (MPC) é uma técnica de controle avançado amplamente utilizada na indústria de processos químicos. Por outro lado, o método de identificação em subespaços (SID) tem se mostrado uma alternativa eficiente para os métodos clássicos de identificação, especialmente para sistemas de ordem elevada com múltiplas saídas e entradas. Da combinação dos conceitos de MPC e SID surgiu o controle preditivo com enfoque em subespaços (SPC), que consiste em um algoritmo de identificação/controle numericamente robusto e facilmente implementável.

Um modelo dinâmico do processo é o primeiro pré-requisito do projeto de um controlador MPC. Tradicionalmente, esse modelo é identificado usando dados amostrados das entradas e das saídas do sistema. Com o modelo obtido, matrizes de predição são construídas, como as matrizes $\Psi$ e $\Theta$ da equação (2.10). Essas matrizes são então utilizadas no controlador para se obter predições de vários passos à frente das saídas. Porém, percebeu-se que essas matrizes de predição podem ser obtidas diretamente do conjunto de dados amostrados, a partir de algumas matrizes usadas no método SID. Dessa forma, é possível projetar um controlador preditivo sem a identificação prévia de um modelo paramétrico.

O SPC permite que os três passos do projeto de um controlador preditivo, isto é, a identificação do modelo, o cálculo do observador de estados (filtro de Kalman) e a construção das matrizes de predição, sejam substituídos por um único passo referente a uma decomposição QR. Além disso, esse procedimento é baseado em conceitos do SID, que envolve a minimização do somatório dos erros de predição vários passos à frente, tornando-o conveniente para o uso em projetos de controle preditivo (HUANG; KADALI, 2008). A Figura 4.1 ilustra esquematicamente as etapas do projeto de um controlador MPC tradicional e do controlador SPC.

No enfoque usual de controle preditivo, o vetor de estados é usado como uma ponte de interligação entre o passado e o futuro. O método SID utiliza essa idéia, obtendo 
uma seqüência de estados antes de se determinar as matrizes do sistema. O SPC também explora esse princípio (FAVOREEL et al., 1999). Pode-se dizer ainda que na equação de predição do SPC o vetor de estados é substituído por dados amostrados do passado. Assim, no MPC com modelo em espaço de estados, as saídas futuras dependem do estado atual e das entradas futuras, enquanto que no SPC, as saídas futuras dependem das entradas e saídas passadas e das entradas futuras.
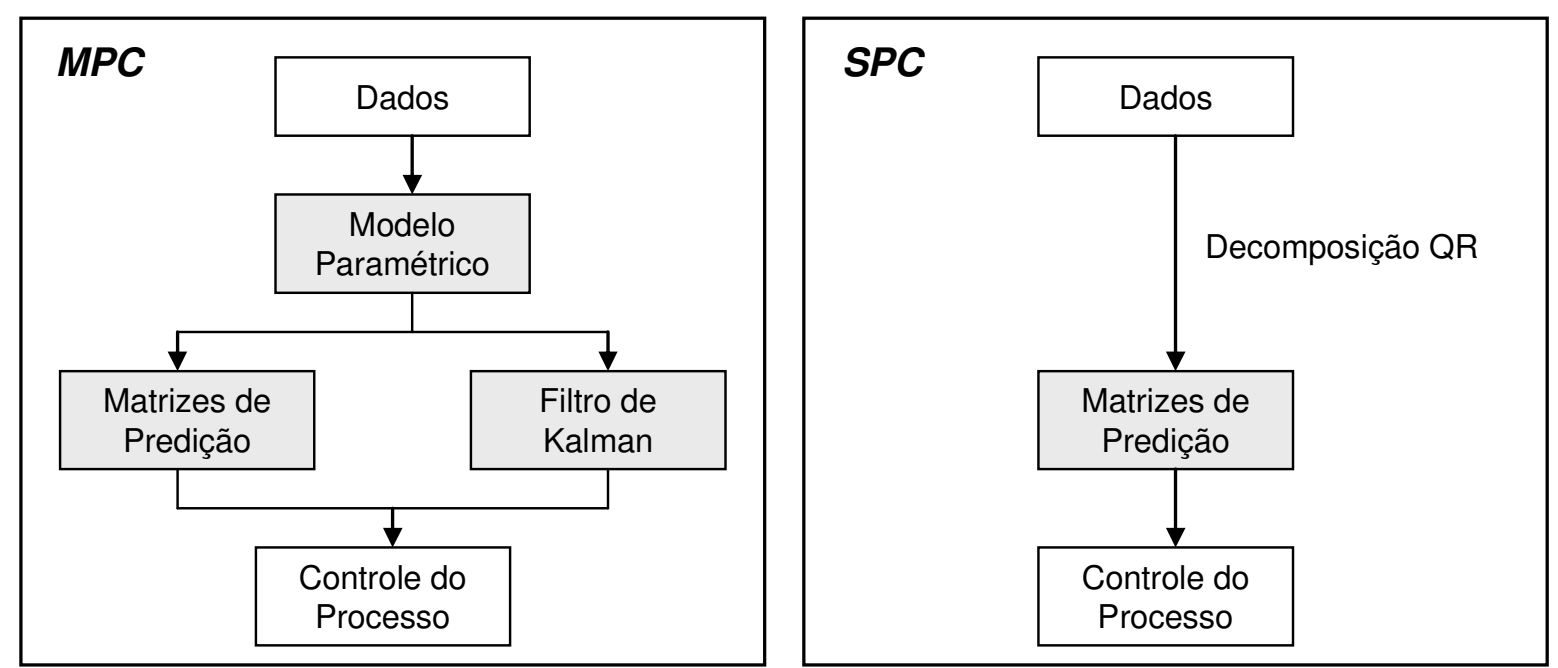

Figura 4.1 - Projeto de um controlador MPC tradicional e do controlador SPC.

A técnica de controle aqui denominada "SPC" tem sido referenciada na literatura de maneiras diferentes. Por não utilizar um modelo paramétrico na forma tradicional, alguns autores adotaram o nome "model-free approach" (enfoque sem modelo). Outros adotaram o nome "data-driven predictive control" (controle preditivo orientado a dados).

A idéia de utilizar um modelo de predição identificado no enfoque de subespaços surgiu no final da década de 90, com os trabalhos de Di Ruscio (1997a; 1997b) e Di Ruscio e Foss (1998). O conceito do SPC da maneira como será tratado neste trabalho foi apresentado por Favoreel e De Moor (1998), para o controle com ação posicional e sem restrições explícitas nas entradas e saídas. Um desenvolvimento com esse enfoque para o projeto de um controlador do tipo LQG (do inglês, Linear Quadratic Gaussian) foi proposto por Favoreel et al. (1999). Esses dois últimos estudos são também tratados de forma detalhada em Favoreel (1999). Kadali; Huang e Rossiter (2003) introduziram o conceito de SPC para o caso de controle 
com ação integral e restrições nas entradas e saídas, aplicando o controlador proposto em uma planta piloto com dinâmica não-linear. $\mathrm{Na}$ área de controle preditivo orientado a dados, pode-se ainda citar os trabalhos de Song; Yoo e Rhee (2001), Hale e Qin (2002) e Wang; Huang e Chen (2007). Finalmente, Huang e Kadali (2008) publicaram um livro sobre o assunto.

As matrizes de predição usadas no SPC e identificadas diretamente a partir de dados amostrados podem ser vistas como um tipo de modelo. Esse enfoque será utilizado aqui e a equação de predição do controlador será chamada de modelo de predição em subespaços. Os artigos publicados e citados no parágrafo acima consideram que essas matrizes são recalculadas a cada período de amostragem. Porém, neste trabalho será considerado que o cálculo das matrizes de predição é realizado uma única vez durante o projeto do controlador ou quando for necessária uma reidentificação.

A seguir (seção 4.1) será descrito o modelo de predição utilizado no SPC e como esse modelo pode ser obtido. Posteriormente, na seção 4.2, será apresentada a metodologia usada no controlador e algumas melhorias. Finalmente, na seção 4.3, o SPC é aplicado na coluna debutanizadora vista anteriormente.

\subsection{MODELO DE PREDIÇÃO EM SUBESPAÇOS}

\subsubsection{Descrição do modelo de predição}

O modelo de predição em subespaços representa as saídas futuras como uma combinação linear do passado e das entradas futuras. Para um sistema com $\mathrm{m}$ entradas e $l$ saídas e com um horizonte de predição igual a $M$, esse modelo pode ser escrito como:

$$
\hat{y}_{f}=L_{w} w_{p}+L_{u} u_{f}
$$

sendo: 
$w_{p}=\left[\begin{array}{c}y_{p} \\ u_{p}\end{array}\right] ; \hat{y}_{f}=\left[\begin{array}{c}\hat{y}(k+1) \\ \hat{y}(k+2) \\ \vdots \\ \hat{y}(k+M)\end{array}\right] ; u_{f}=\left[\begin{array}{c}u(k+1) \\ u(k+2) \\ \vdots \\ u(k+M)\end{array}\right] ; y_{p}=\left[\begin{array}{c}y(k-M+1) \\ y(k-M+2) \\ \vdots \\ y(k)\end{array}\right] ; u_{p}=\left[\begin{array}{c}u(k-M+1) \\ u(k-M+2) \\ \vdots \\ u(k)\end{array}\right]$;

$\hat{y}_{f} \in R^{l . M} \quad$ vetor de predição das saídas futuras;

$u_{f} \in R^{m . M} \quad$ vetor com as entradas futuras;

$y_{p} \in R^{l . M} \quad$ vetor com as saídas passadas;

$u_{p} \in R^{m . M} \quad$ vetor com as entradas passadas;

$w_{p} \in R^{(m+l) \cdot M}$ vetor conjunto das saídas e entradas passadas;

$L_{w} \in R^{l . M \times(m+l) . M}$ matriz do modelo referente ao passado;

$L_{u} \in R^{l . M \times m . M}$ matriz do modelo referente às entradas futuras;

M horizonte referente à predição e ao passado usado no modelo.

Note que o modelo de predição em (4.1) equivale à primeira coluna de dados calculada pela equação (3.45), apresentada durante o desenvolvimento do método SID. Portanto, as matrizes $L_{w}$ e $L_{u}$ desse modelo são as mesmas matrizes da equação (3.45) e podem ser calculadas a partir de um conjunto de dados amostrados. Esse cálculo é feito através da projeção $Z_{i}$, definida em (3.39), utilizando a relação apresentada em (3.47):

$$
Z_{i}=Y_{f} /\left[\begin{array}{l}
W_{p} \\
U_{f}
\end{array}\right]=\left[\begin{array}{ll}
L_{w} & L_{u}
\end{array}\right]\left[\begin{array}{c}
W_{p} \\
U_{f}
\end{array}\right]
$$

Vale lembrar que, na equação acima, todas as matrizes de dados são compostas por dados passados previamente coletados. Entretanto, no modelo de predição em subespaços (4.1), os componentes $u_{f}$ e $\hat{y}_{f}$ de fato referem-se a dados futuros e representam, respectivamente, as ações que serão inseridas na planta e a predição da resposta do processo devido a essas ações. 
O horizonte de predição do modelo $(M)$ é também utilizado como o horizonte no qual os dados passados são informados para a determinação do estado atual do sistema. Além disso, o número de blocos de linhas $(i)$ das matrizes de Hankel usadas na identificação das matrizes de predição deve ser igual a $M$. No capítulo anterior discutiu-se que $i$ e, portanto, também $M$ devem ser maiores que a ordem do sistema $(n x)$. Contudo, para que os dados passados representem com exatidão o estado do sistema, é necessário que o horizonte referente ao passado tenda ao infinito (FAVOREEL; DE MOOR, 1998).

Uma observação importante é que a matriz $L_{u}$ da equação (4.1) sempre será uma matriz triangular inferior, pois caso contrário, a saída em determinado instante dependeria de ações inseridas em instantes posteriores, o que é impossível. Por fim, analisando o primeiro bloco de linhas da equação de predição em subespaços, percebe-se que esse modelo obedece à seguinte estrutura ARX:

$$
y(t)=-a_{1} y(t-1)-\ldots-a_{M} y(t-M)+b_{0} u(t)+b_{1} u(t-1)+\ldots+b_{M} u(t-M)+e(t)
$$

A equivalência entre a estrutura $A R X$ e o modelo de predição em subespaços somente é verdadeira quando o número de dados usados na identificação $(N)$ e 0 horizonte do modelo $(M)$ tendem ao infinito (FAVOREEL; DE MOOR, 1998).

\subsubsection{Implementação numérica da identificação}

O cálculo das matrizes $L_{w}$ e $L_{u}$ é realizado através da matriz $Z_{i}$, que consiste em uma projeção das matrizes de blocos de Hankel de entrada e de saída $\left(U_{p}, U_{f}, Y_{p}\right.$ e $\left.Y_{f}\right)$. Essas matrizes, por sua vez, são formadas por dados amostrados do sistema real. A implementação numérica da projeção $Z_{i}$ pode ser realizada através da seguinte decomposição QR: 


$$
\left[\begin{array}{c}
W_{p} \\
U_{f} \\
Y_{f}
\end{array}\right]=[\begin{array}{ccc}
R_{11} & 0 & 0 \\
R_{21} & R_{22} & 0 \\
R_{31} & R_{32} & R_{33}
\end{array} \underbrace{\left[\begin{array}{c}
Q_{1}^{T} \\
Q_{2}^{T} \\
Q_{3}^{T}
\end{array}\right]}_{Q^{T}}
$$

Observe que cada componente da equação acima pode ser escrito separadamente:

$$
\begin{aligned}
& W_{p}=\left[\begin{array}{lll}
R_{11} & 0 & 0
\end{array}\right] Q^{T} \\
& U_{f}=\left[\begin{array}{lll}
R_{21} & R_{22} & 0
\end{array}\right] Q^{T} \\
& Y_{f}=\left[\begin{array}{lll}
R_{31} & R_{32} & R_{33}
\end{array}\right] Q^{T}
\end{aligned}
$$

Pela definição de projeção ortogonal (3.32), pode-se escrever:

$$
\begin{aligned}
& Z_{i}=Y_{f} /\left[\begin{array}{l}
W_{p} \\
U_{f}
\end{array}\right] \\
& =\left[\begin{array}{lll}
R_{31} & R_{32} & R_{33}
\end{array}\right] Q^{T}\left(\left[\begin{array}{rrr}
R_{11} & 0 & 0 \\
R_{21} & R_{22} & 0
\end{array}\right] Q^{T}\right)^{T} \cdot\left(\left[\begin{array}{ccc}
R_{11} & 0 & 0 \\
R_{21} & R_{22} & 0
\end{array}\right] Q^{T}\left(\left[\begin{array}{rrr}
R_{11} & 0 & 0 \\
R_{21} & R_{22} & 0
\end{array}\right] Q^{T}\right)^{T}\right)^{\dagger} \cdot\left[\begin{array}{rrr}
R_{11} & 0 & 0 \\
R_{21} & R_{22} & 0
\end{array}\right] Q^{T}
\end{aligned}
$$

Desenvolvendo a equação acima e sabendo que $Q^{T} Q=I$ ( $Q$ é ortonormal), tem-se:

$$
Z_{i}=\left[\begin{array}{lll}
R_{31} & R_{32} & R_{33}
\end{array}\right]\left[\begin{array}{ccc}
R_{11} & 0 & 0 \\
R_{21} & R_{22} & 0
\end{array}\right]^{T} \cdot\left(\left[\begin{array}{ccc}
R_{11} & 0 & 0 \\
R_{21} & R_{22} & 0
\end{array}\right]\left[\begin{array}{ccc}
R_{11} & 0 & 0 \\
R_{21} & R_{22} & 0
\end{array}\right]^{T}\right)^{\dagger} \cdot\left[\begin{array}{ccc}
R_{11} & 0 & 0 \\
R_{21} & R_{22} & 0
\end{array}\right] Q^{T}
$$

Note que a última coluna da matriz dessa equação resultará em zero e portanto pode-se escrever:

$$
Z_{i}=\left[\begin{array}{ll}
R_{31} & R_{32}
\end{array}\right]\left[\begin{array}{cc}
R_{11} & 0 \\
R_{21} & R_{22}
\end{array}\right]^{T} \cdot\left(\left[\begin{array}{cc}
R_{11} & 0 \\
R_{21} & R_{22}
\end{array}\right]\left[\begin{array}{cc}
R_{11} & 0 \\
R_{21} & R_{22}
\end{array}\right]^{T}\right)^{\dagger} \cdot\left[\begin{array}{cc}
R_{11} & 0 \\
R_{21} & R_{22}
\end{array}\right]\left[\begin{array}{l}
Q_{1}^{T} \\
Q_{2}{ }^{T}
\end{array}\right]
$$

Como as matrizes dentro da pseudo-inversa são quadradas, pode-se substituir essa pseudo-inversa pela inversa: 


$$
\begin{gathered}
Z_{i}=\left[\begin{array}{ll}
R_{31} & R_{32}
\end{array}\right] \overbrace{\left[\begin{array}{ll}
R_{11} & 0 \\
R_{21} & R_{22}
\end{array}\right]^{T}\left[\begin{array}{cc}
R_{11} & 0 \\
R_{21} & R_{22}
\end{array}\right]^{-T}}^{=I} \cdot\left[\begin{array}{cc}
R_{11} & 0 \\
R_{21} & R_{22}
\end{array}\right]^{-1} \cdot\left[\begin{array}{cc}
R_{11} & 0 \\
R_{21} & R_{22}
\end{array}\right]\left[\begin{array}{c}
Q_{1}{ }^{T} \\
Q_{2}{ }^{T}
\end{array}\right] \\
=\left[\begin{array}{ll}
R_{31} & R_{32}
\end{array}\right] \cdot\left[\begin{array}{cc}
R_{11} & 0 \\
R_{21} & R_{22}
\end{array}\right]^{-1} \cdot\left[\begin{array}{cc}
R_{11} & 0 \\
R_{21} & R_{22}
\end{array}\right]\left[\begin{array}{c}
Q_{1}{ }^{T} \\
Q_{2}{ }^{T}
\end{array}\right]
\end{gathered}
$$

Pela equação (3.47) sabe que a projeção $Z_{i}$ é dada pelo produto das matrizes de predição com o conjunto das matrizes de Hankel dos dados passados (entradas e saídas) e das entradas futuras. Observe ainda que as duas últimas matrizes do lado direito da equação (4.3) correspondem às matrizes de Hankel:

$$
\left[\begin{array}{l}
W_{p} \\
U_{f}
\end{array}\right]=\left[\begin{array}{cc}
R_{11} & 0 \\
R_{21} & R_{22}
\end{array}\right]\left[\begin{array}{l}
Q_{1}{ }^{T} \\
Q_{2}{ }^{T}
\end{array}\right]
$$

Logo, pode-se determinar as matrizes de predição por:

$$
\left[\begin{array}{ll}
L_{w} & L_{u}
\end{array}\right]=\left[\begin{array}{ll}
R_{31} & R_{32}
\end{array}\right] \cdot\left[\begin{array}{cc}
R_{11} & 0 \\
R_{21} & R_{22}
\end{array}\right]^{-1}
$$

É importante ressaltar que a identificação das matrizes de predição surgiu dos conceitos do SID e, portanto, as mesmas hipóteses são consideradas. Logo, devese utilizar um número de dados grande o suficiente $(N \rightarrow \infty)$ e o número de blocos de linhas das matrizes de Hankel $(i)$, que é igual ao horizonte do modelo $(M)$, deve ser muito menor que o número de colunas $(j)$.

A decomposição QR necessária na obtenção das matrizes é uma ferramenta numérica da álgebra linear bastante robusta e bem difundida em softwares de matemática. O Matlab, por exemplo, contem um comando que, dada uma matriz, ele realiza essa decomposição automaticamente.

A fim de verificar a validade do modelo de predição em subespaços e do algoritmo de identificação proposto para esse modelo, foram realizadas simulações com o sistema da coluna debutanizadora da refinaria RPBC. Considerou-se que a planta é 
representada pelo Modelo C1. A resposta ao degrau do sistema real foi comparada com a resposta ao degrau dada pelo modelo de predição identificado. Analisaram-se dois casos: dados amostrados sem ruído e dados amostrados com ruído randômico de variância 0.001 adicionado à saída do processo. Nos procedimentos de identificação foram usados os dados obtidos nos experimentos $A$ e $D$ (Tabela 3.4) do capítulo anterior. Cada identificação utilizou 1500 pontos.

A resposta ao degrau do modelo de predição em subespaços pode ser obtida de duas maneiras diferentes. A primeira consiste em um único cálculo, no qual se insere o degrau no vetor de entradas futuras $u_{f}$ e a resposta do processo em um horizonte $M$ é dada pelo vetor de saídas futuras $\hat{y}_{f}$. Na segunda maneira, a predição é realizada considerando-se apenas os primeiros componentes dos vetores de dados futuros, referentes ao próximo período de amostragem. A cada instante, esses elementos são substituídos no vetor de dados passados, eliminando-se os dados mais antigos. Como no controle preditivo o vetor de dados passados é atualizado a cada instante de amostragem e o cálculo das saídas futuras é feito recursivamente, optou-se por simular a resposta ao degrau através do segundo método.

Para cada um dos dois casos descritos anteriormente (dados amostrados com e sem ruído), diversas identificações foram realizadas alterando-se o valor do parâmetro $M$. Os resultados das respostas ao degrau dos modelos obtidos e do sistema real estão mostrados na Figura 4.2, para o caso sem ruído, e na Figura 4.3, para o caso com ruído de variância 0.001 .

Para o caso de dados de identificação sem ruído (Figura 4.2), a grande maioria dos modelos apresentou resposta ao degrau idêntica ao sistema original, validando o modelo e método de identificação propostos. A única exceção ocorreu para o modelo com o horizonte $M=3$, que apresentou bias no ganho de algumas variáveis. Isso ocorreu, pois para esse caso o valor de $M$ é menor que a ordem do sistema, sendo que o modelo de predição não possui informação suficiente acerca do passado para determinar o estado atual do sistema. 

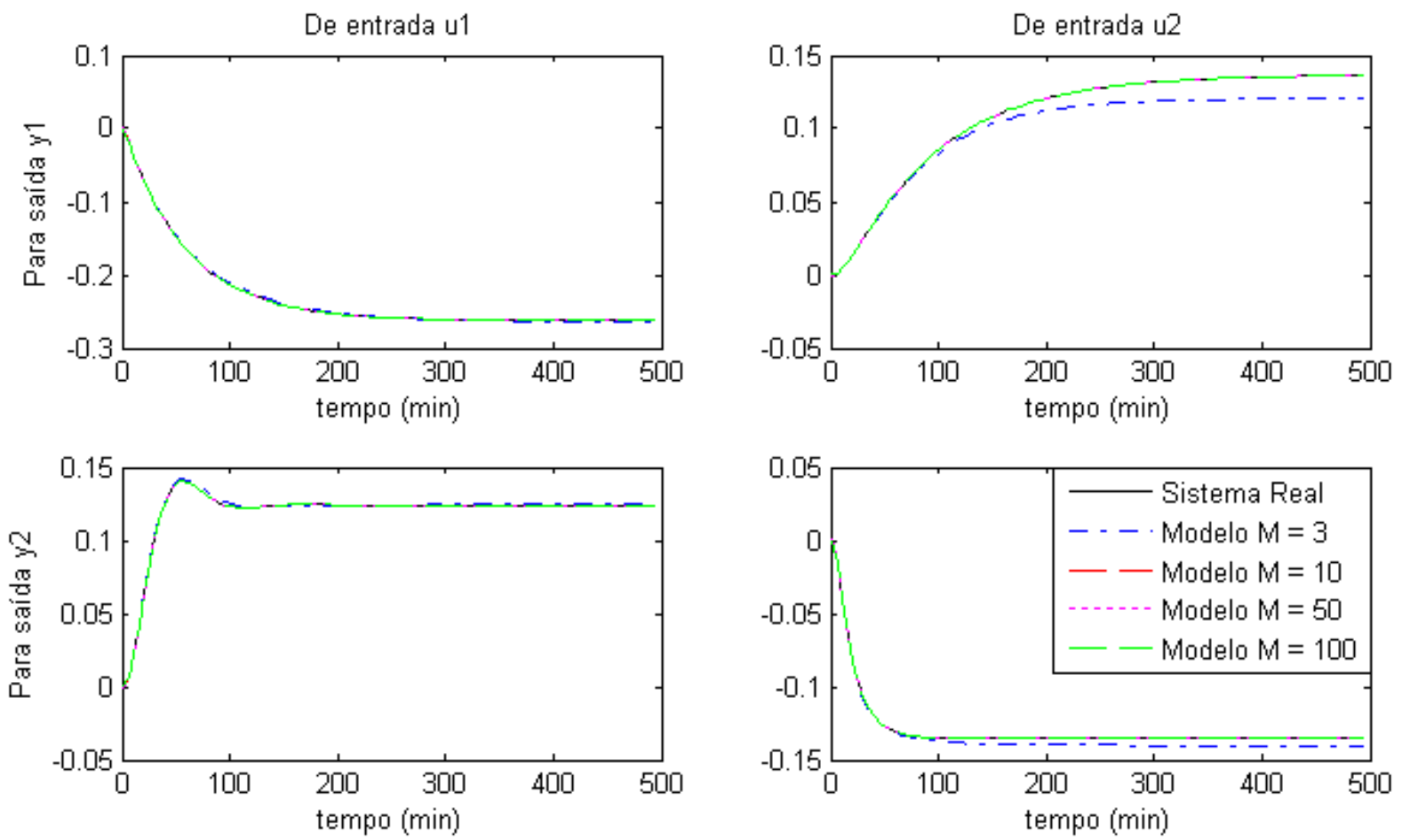

Figura 4.2 - Comparação das respostas ao degrau do sistema real da coluna debutanizadora e dos modelos de predição obtidos pela identificação com dados sem ruído.
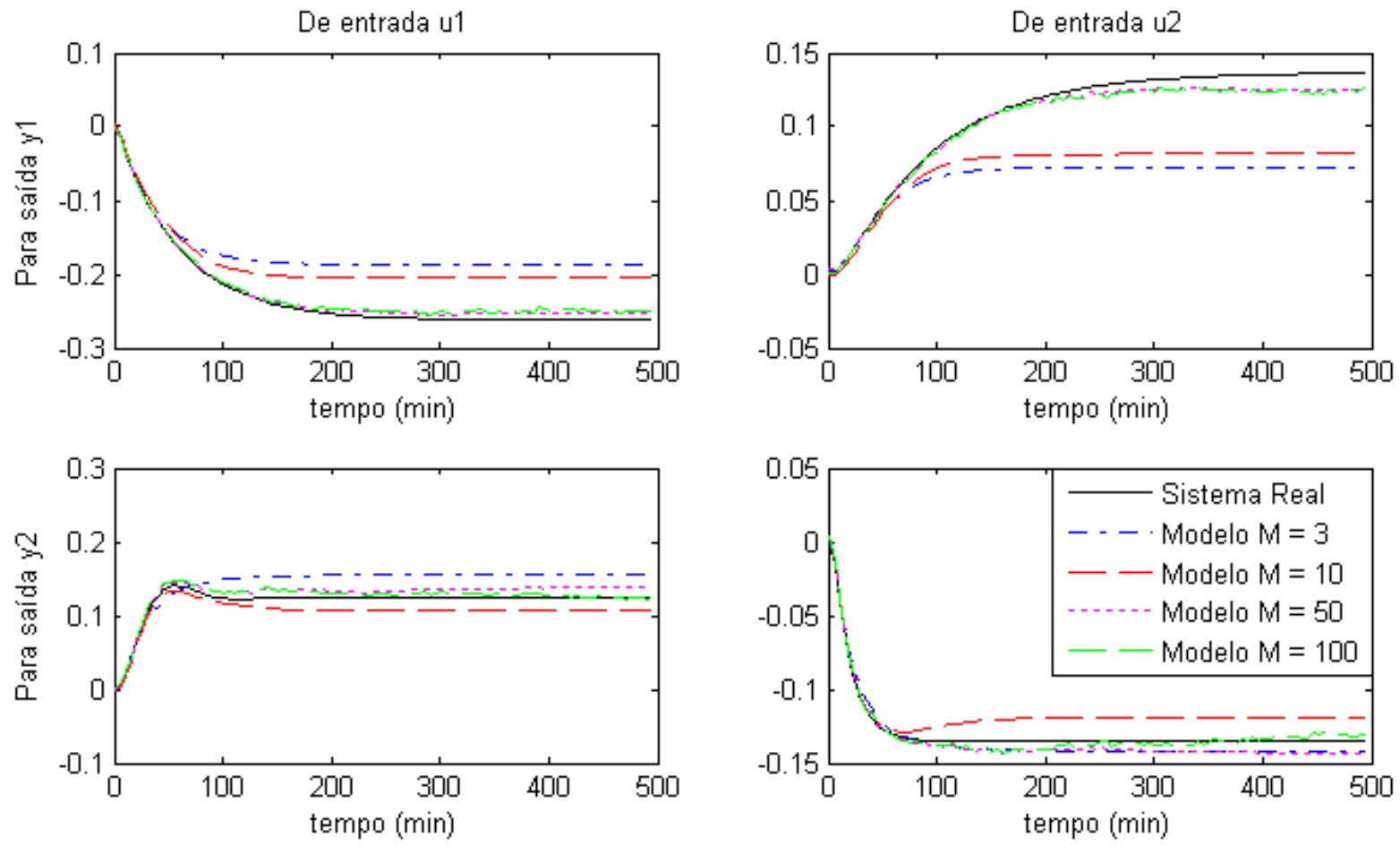

Figura 4.3 - Comparação das respostas ao degrau do sistema real da coluna debutanizadora e dos modelos de predição obtidos pela identificação com dados com ruído de variância 0.001 .

Na Figura 4.3, nota-se que quando há ruído nos dados usados na identificação, a influência do parâmetro $M$ é bem maior. Para valores de $M$ muito baixos, observase que o modelo identificado possui um desempenho ruim. Contudo, se o horizonte 
$M$ for muito elevado, percebe-se o surgimento de problemas devido à sobreparametrização do modelo.

\subsubsection{Relação com modelos em espaço de estados}

A partir do modelo em espaço de estados (3.12)-(3.13), é possível encontrar equações que relacionam as matrizes $A, B, C$ e $D$ com as matrizes de predição $L_{w}$ e $L_{u}$. Como o próprio nome indica, o modelo de predição em subespaços tem a função de prever o comportamento do processo com base em informações do passado e das entradas futuras. Logo, para se encontrar a relação entre esse

modelo e o modelo em espaço de estados, é necessário considerar apenas a parcela determinística do último, já que a parte estocástica (ruídos não medidos $v(k)$ e $w(k))$ não pode ser prevista.

Considerando-se um horizonte $M$, pode-se escrever a parcela determinística das equações (3.21)-(3.23) para apenas a primeira coluna das matrizes de dados de Hankel, como:

$$
\begin{aligned}
& y_{p}=\Gamma_{M} x^{d}(k-M)+H_{M}^{d} u_{p} \\
& y_{f}=\Gamma_{M} x^{d}(k)+H_{M}^{d} u_{f} \\
& x^{d}(k)=A^{M} x^{d}(k-M)+\Delta_{M}^{d} u_{p}
\end{aligned}
$$

sendo:

$$
\begin{aligned}
& \Delta_{M}^{d}=\left[\begin{array}{lllll}
A^{M-1} B & A^{M-2} B & \cdots & A B & B
\end{array}\right] ; \\
& \Gamma_{M}=\left[\begin{array}{c}
C \\
C A \\
\vdots \\
C A^{M-1}
\end{array}\right] ; \quad H_{M}^{d}=\left[\begin{array}{cccc}
D & 0 & \cdots & 0 \\
C B & D & \cdots & 0 \\
\vdots & \vdots & \ddots & \vdots \\
C A^{M-2} B & C A^{M-3} B & \cdots & D
\end{array}\right] .
\end{aligned}
$$

Substituindo a equação (4.7) na equação (4.6), tem-se: 


$$
y_{f}=\Gamma_{M}\left(A^{M} x^{d}(k-M)+\Delta_{M}^{d} u_{p}\right)+H_{M}^{d} u_{f}
$$

A partir da equação (4.5) pode-se escrever:

$$
x^{d}(k-M)=\Gamma_{M}^{\dagger} y_{p}-\Gamma_{M}^{\dagger} H_{M}^{d} u_{p}
$$

A combinação das equações (4.8) e (4.9) resulta em:

$$
\begin{aligned}
y_{f}= & \Gamma_{M}\left(A^{M} \Gamma_{M}^{\dagger} y_{p}-A^{M} \Gamma_{M}^{\dagger} H_{M}^{d} u_{p}+\Delta_{M}^{d} u_{p}\right)+H_{M}^{d} u_{f} \\
& =\Gamma_{M}\left[\begin{array}{ll}
A^{M} \Gamma_{M}^{\dagger} & \Delta_{M}^{d}-A^{M} \Gamma_{M}^{\dagger} H_{M}^{d}
\end{array}\right]\left[\begin{array}{l}
y_{p} \\
u_{p}
\end{array}\right]+H_{M}^{d} u_{f} \\
& =L_{w} w_{p}+L_{u} u_{f}
\end{aligned}
$$

Portanto:

$$
\begin{aligned}
& L_{w}=\Gamma_{M}\left[A^{M} \Gamma_{M}^{\dagger} \Delta_{M}^{d}-A^{M} \Gamma_{M}^{\dagger} H_{M}^{d}\right] \\
& L_{u}=H_{M}^{d}
\end{aligned}
$$

Através das equações (4.10) e (4.11) é possível determinar as matrizes do modelo de predição em subespaços a partir do modelo em espaço de estados.

Um resultado importante relacionado ao modelo de predição em subespaços, mostrado em Favoreel e De Moor (1998), é que, quando o horizonte $M \rightarrow \infty$, a predição das saídas futuras passa a ser representada pela equação (4.6) e, portanto:

$$
\begin{aligned}
& L_{w} w_{p}=\Gamma_{M} x^{d}(k) \\
& L_{u}=H_{M}^{d}
\end{aligned}
$$

Substituindo as relações acima no modelo de predição em subespaços obtém-se exatamente a equação de predição utilizada no controlador MPC posicional. Por esse motivo, pode-se afirmar que, quando $M \rightarrow \infty$, o SPC (controlador que utiliza o 
modelo de predição em subespaços) equivale ao MPC. O termo $L_{w} w_{p}=\Gamma_{M} x^{d}(k)$ representa a resposta "livre" do modelo, isto é, o comportamento do sistema caso nenhuma perturbação (entrada ou ruído) seja aplicada, enquanto que a matriz $L_{u}$ representa a resposta ao degrau.

As equações (4.10) e (4.11) foram testadas para o sistema da coluna debutanizadora. A partir do modelo em espaço de estados correspondente ao Modelo $\mathrm{C} 1$, as matrizes $L_{w}$ e $L_{u}$ foram calculadas, considerando-se diferentes valores de $M$. As respostas ao degrau dos modelos de predição obtidos e do sistema real foram comparadas. O resultado está mostrado na Figura 4.4. Percebese que o comportamento dos modelos de predição obtidos a partir das matrizes do modelo em espaço de estados é muito semelhante ao comportamento dos modelos de predição identificados no caso de dados amostrados sem ruído (Figura 4.2). Todos os modelos, com exceção de $M=3$ (que é menor que a ordem do sistema), obtiveram resposta idêntica ao sistema real.
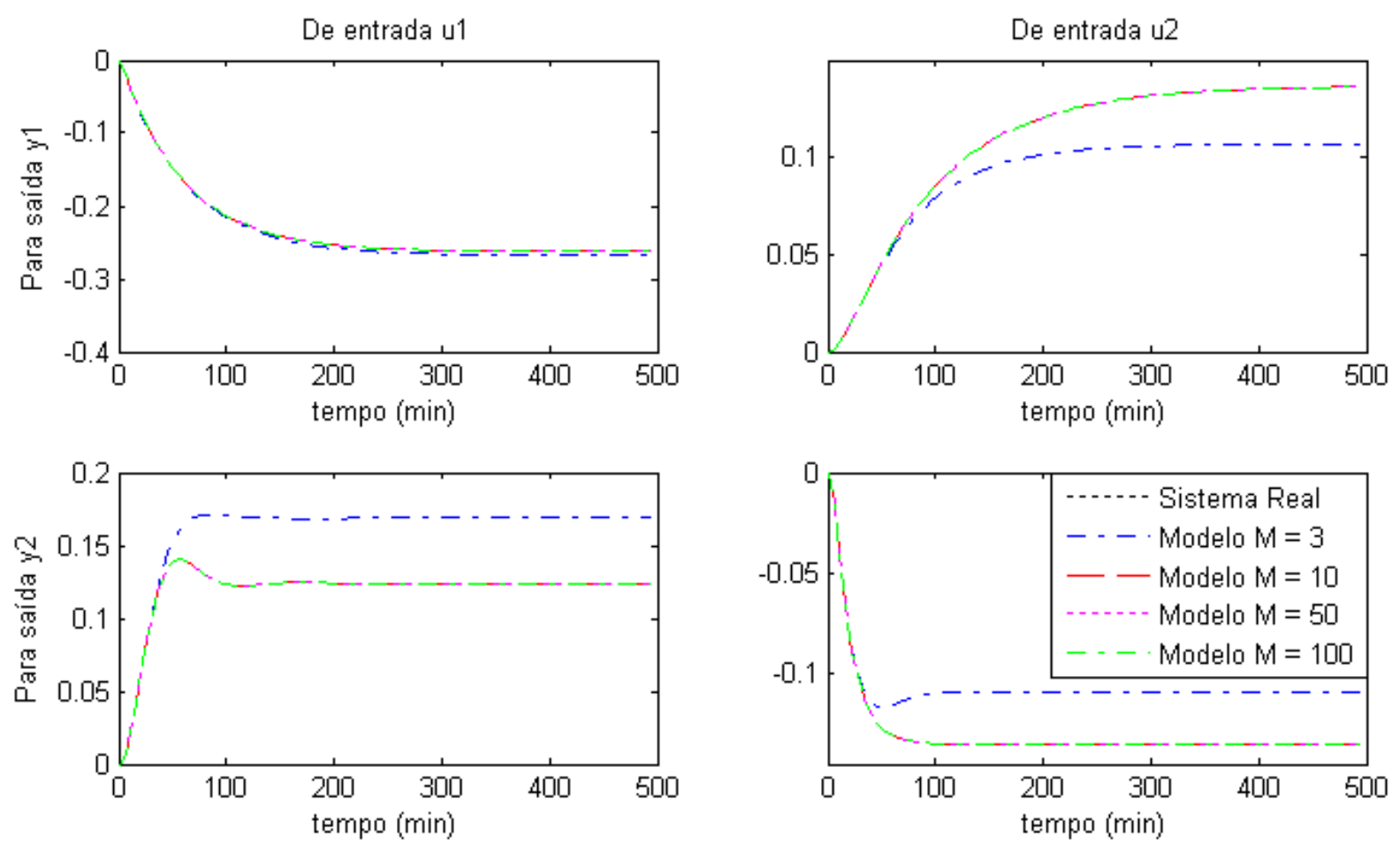

Figura 4.4 - Comparação das respostas ao degrau do sistema real da coluna debutanizadora e dos modelos de predição obtidos a partir do modelo em espaço de estados. 


\subsubsection{Obtenção do modelo de predição incremental na entrada}

Quando um controlador preditivo é desenvolvido com um modelo na forma posicional na entrada, o controle resultante apresenta off-set, ou seja, as variáveis controladas não alcançam seus respectivos set-points. Por esse motivo, é importante tratar o sistema de forma incremental.

É possível se obter um modelo de predição em subespaços na forma incremental a partir do modelo posicional apresentado na equação (4.1). Para isso, deve-se primeiramente considerar os vetores de saídas e de entradas na forma incremental. Definindo $\Delta u(t)=u(t)-u(t-1)$ e $\Delta y(t)=y(t)-y(t-1)$, é fácil demonstrar que:

$$
\Delta \hat{y}_{f}=L_{w} \Delta w_{p}+L_{u} \Delta u_{f}
$$

sendo:

$$
\begin{aligned}
& \Delta w_{p}=\left[\begin{array}{c}
\Delta y_{p} \\
\Delta u_{p}
\end{array}\right] ; \quad \Delta \hat{y}_{f}=\left[\begin{array}{c}
\Delta \hat{y}(k+1) \\
\Delta \hat{y}(k+2) \\
\vdots \\
\Delta \hat{y}(k+M)
\end{array}\right] ; \quad \Delta u_{f}=\left[\begin{array}{c}
\Delta u(k+1) \\
\Delta u(k+2) \\
\vdots \\
\Delta u(k+M)
\end{array}\right] ; \\
& \Delta y_{p}=\left[\begin{array}{c}
\Delta y(k-M+1) \\
\Delta y(k-M+2) \\
\vdots \\
\Delta y(k)
\end{array}\right] ; \quad \Delta u_{p}=\left[\begin{array}{c}
\Delta u(k-M+1) \\
\Delta u(k-M+2) \\
\vdots \\
\Delta u(k)
\end{array}\right] .
\end{aligned}
$$

Separando as matrizes $L_{w}$ e $L_{u}$ em $M$ blocos de linhas, conforme mostrado abaixo, pode-se escrever:

$$
\left[\begin{array}{c}
\Delta \hat{y}(k+1) \\
\Delta \hat{y}(k+2) \\
\vdots \\
\Delta \hat{y}(k+M)
\end{array}\right]=\left[\begin{array}{c}
L_{w}{ }^{(1)} \\
L_{w}{ }^{(2)} \\
\vdots \\
L_{w}{ }^{(M)}
\end{array}\right] \Delta w_{p}+\left[\begin{array}{c}
L_{u}{ }^{(1)} \\
L_{u}{ }^{(2)} \\
\vdots \\
L_{u}{ }^{(M)}
\end{array}\right] \Delta u_{f}
$$

Os blocos de linhas da equação acima podem ser escritos separadamente: 


$$
\begin{aligned}
& \Delta \hat{y}(k+1)=L_{w}{ }^{(1)} \Delta w_{p}+L_{u}{ }^{(1)} \Delta u_{f} \\
& \Delta \hat{y}(k+2)=L_{w}{ }^{(2)} \Delta w_{p}+L_{u}{ }^{(2)} \Delta u_{f} \\
& \quad \vdots \\
& \Delta \hat{y}(k+M)=L_{w}{ }^{(M)} \Delta w_{p}+L_{u}{ }^{(M)} \Delta u_{f}
\end{aligned}
$$

Logo, o vetor de predições das saídas futuras pode ser representado por:

$$
\begin{aligned}
& \hat{y}_{f}=\left[\begin{array}{c}
\hat{y}(k+1) \\
\hat{y}(k+2) \\
\vdots \\
\hat{y}(k+M)
\end{array}\right]=\left[\begin{array}{c}
y(k) \\
y(k) \\
\vdots \\
y(k)
\end{array}\right]+\left[\begin{array}{c}
\Delta \hat{y}(k+1) \\
\Delta \hat{y}(k+1)+\Delta \hat{y}(k+2) \\
\vdots \\
\Delta \hat{y}(k+1)+\ldots+\Delta \hat{y}(k+M)
\end{array}\right] \\
& =Y_{k}+\left[\begin{array}{c}
L_{w}{ }^{(1)} \Delta w_{p}+L_{u}{ }^{(1)} \Delta u_{f} \\
L_{w}{ }^{(1)} \Delta w_{p}+L_{u}{ }^{(1)} \Delta u_{f}+L_{w}{ }^{(2)} \Delta w_{p}+L_{u}{ }^{(2)} \Delta u_{f} \\
\vdots \\
L_{w}{ }^{(1)} \Delta w_{p}+L_{u}{ }^{(1)} \Delta u_{f}+\ldots+L_{w}{ }^{(M)} \Delta w_{p}+L_{u}{ }^{(M)} \Delta u_{f}
\end{array}\right]
\end{aligned}
$$

Essa equação pode ser organizada da seguinte forma:

$$
\hat{y}_{f}=Y_{k}+\underbrace{\left[\begin{array}{c}
L_{w}{ }^{(1)} \\
L_{w}^{(1)}+L_{w}{ }^{(2)} \\
\vdots \\
L_{w}{ }^{(1)}+\ldots+L_{w}{ }^{(M)}
\end{array}\right]}_{L_{w}^{\text {ic }}} \Delta w_{p}+\underbrace{\left[\begin{array}{c}
L_{u}{ }^{(1)} \\
L_{u}^{(1)}+L_{u}{ }^{(2)} \\
\vdots \\
L_{u}^{(1)}+\ldots+L_{u}{ }^{(M)}
\end{array}\right]}_{L_{u}^{\text {inc }}} \Delta u_{f}
$$

Finalmente obtém-se o modelo de predição em subespaços na forma incremental:

$$
\hat{y}_{f}=Y_{k}+L_{w}^{i n c} \Delta w_{p}+L_{u}^{i n c} \Delta u_{f}
$$

O sobrescrito inc nas matrizes do modelo acima significa "incremental". Observe que essas matrizes podem ser facilmente obtidas a partir das matrizes do modelo posicional pelas seguintes equações:

$$
\begin{aligned}
& L_{w}^{i n c}=\Pi L_{w} \\
& L_{u}^{i n c}=\Pi L_{u}
\end{aligned}
$$


sendo:

$$
\Pi=\left[\begin{array}{cccc}
I_{l} & 0 & \cdots & 0 \\
I_{l} & I_{l} & \cdots & 0 \\
\vdots & \vdots & \ddots & \vdots \\
I_{l} & I_{l} & \cdots & I_{l}
\end{array}\right]
$$

O modelo de predição em subespaços na forma incremental será o modelo utilizado no desenvolvimento do controlador SPC.

\subsection{METODOLOGIA DO CONTROLADOR}

Nesta seção será apresentado o método de controle preditivo com enfoque em subespaços (SPC). Essa técnica utiliza o modelo de predição em subespaços para representar a dinâmica do processo. Será estudado o SPC com modelo incremental na entrada e nos dados passados, conforme equação (4.12). Na seção 4.2.1 é apresentado o algoritmo de controle original do SPC, enquanto que na seção 4.2.2 são introduzidas algumas melhorias no método.

\subsubsection{SPC original com horizonte único}

O problema de otimização a ser resolvido pelo SPC a cada período de amostragem é análogo ao problema resolvido pelo MPC. A única diferença é que os horizontes de predição e de controle não podem ser escolhidos livremente. Em razão do modelo de predição utilizado, esses horizontes devem ser iguais ao horizonte $M$ do modelo. Assim, o problema de otimização pode ser escrito como: 


$$
\min _{\Delta u_{f}} J_{k}=\sum_{j=1}^{M}\left(y(k+j \mid k)-y^{S P}\right)^{T} Q\left(y(k+j \mid k)-y^{S P}\right)+\sum_{j=1}^{M} \Delta u(k+j \mid k)^{T} R \Delta u(k+j \mid k)
$$

sujeito a:

$$
\begin{aligned}
& -\Delta u_{\max } \leq \Delta u(k+j \mid k) \leq \Delta u_{\max }, \quad j=1, \ldots, M \\
& u_{\min } \leq u(k+j \mid k) \leq u_{\max }, \quad j=1, \ldots, M
\end{aligned}
$$

Note que no problema acima as ações de controle a serem calculadas iniciam-se no instante $k+1$, diferentemente do MPC, no qual a primeira ação de controle refere-se ao instante atual $k$. Isso acontece em virtude da identificação do modelo de predição ser baseada em um sistema representado pelo modelo em espaço de estados (3.12)-(3.13) com a matriz $D$ não-nula. Assim, é considerado um processo com ação direta da entrada na saída, ou seja, a saída no instante $k$ é dependente da entrada nesse mesmo instante. Portanto, se a saída $y(k)$ é conhecida, a próxima ação de controle que pode ser calculada é $u(k+1)$.

A partir da equação (4.12) do modelo de predição em subespaços, a função objetivo definida em (4.15) pode ser escrita como:

$$
J_{k}=\left(Y_{k}+L_{w}^{i n c} \Delta w_{p}+L_{u}^{i n c} \Delta u_{f}-Y_{s p}\right)^{T} \bar{Q}\left(Y_{k}+L_{w}^{i n c} \Delta w_{p}+L_{u}^{i n c} \Delta u_{f}-Y_{s p}\right)+\Delta u_{f}{ }^{T} \bar{R} \Delta u_{f}
$$

As entradas futuras $\left(u_{f}\right)$ podem ser escritas em função de suas variações $\left(\Delta u_{f}\right)$. Realizando um desenvolvimento análogo ao realizado para o método MPC e considerando que a última entrada conhecida é $u(k)$, tem-se que:

$$
u_{f}=\bar{I} u(k)+\bar{M} \Delta u_{f}
$$

Utilizando as equações (4.18) e (4.19), o problema de otimização representado pela função objetivo (4.15) e pelas restrições (4.16) e (4.17) pode ser escrito na forma quadrática como:

$$
\begin{aligned}
& \min _{\Delta u_{f}} J_{k}=\Delta u_{f}{ }^{T} H_{2} \Delta u_{f}+2 c_{f 2}{ }^{T} \Delta u_{f}+c_{2} \\
& \text { sujeito a: } A_{\text {ineq } 2} \Delta u_{f} \leq b_{\text {ineq } 2}
\end{aligned}
$$


sendo:

$$
\begin{aligned}
& H_{2}=L_{u}^{i n c^{T}} \bar{Q} L_{u}^{i n c}+\bar{R} \\
& c_{f 2}{ }^{T}=\left[Y_{k}+L_{w}^{i n c} \Delta w_{p}-Y_{s p}\right]^{T} \bar{Q} L_{u}^{i n c} ; \\
& c_{2}=\left[Y_{k}+L_{w}^{i n c} \Delta w_{p}-Y_{s p}\right]^{T} \bar{Q}\left[Y_{k}+L_{w}^{i n c} \Delta w_{p}-Y_{s p}\right] \text {; }
\end{aligned}
$$

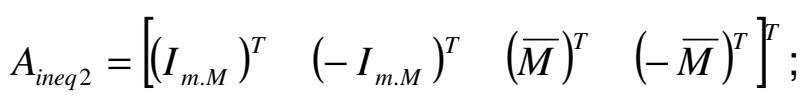

$$
\begin{aligned}
& b_{\text {ineq } 2}=\left[\begin{array}{lll}
\left(\bar{I} \Delta u_{\max }\right)^{T} & \left(-\bar{I} \Delta u_{\max }\right)^{T} \quad\left(\bar{I}\left(u_{\max }-u(k)\right)\right)^{T} \quad\left(\bar{I}\left(u(k)-u_{\text {min }}\right)\right)^{T}
\end{array}\right]^{T} .
\end{aligned}
$$

As matrizes $\bar{Q}, \bar{R}, Y_{s p}, \bar{M}$ e $\bar{I}$ são definidas pelas mesmas equações usadas no MPC, com uma única diferença: os valores dos horizontes de predição $(n p)$ e de controle $(n c)$ devem ser substituídos pelo horizonte $M$ do modelo de predição em subespaços.

A cada iteração, o problema de otimização descrito em (4.15)-(4.17) é resolvido e somente a primeira ação de controle $(u(k+1))$ é inserida na planta. As saídas do processo são então medidas e os dados até o instante atual ( $k$ ) são armazenados. No instante posterior, todo o procedimento é repetido. Considera-se, neste trabalho, que as matrizes $L_{w}$ e $L_{u}$ do modelo de predição em subespaços são, a princípio, mantidas constantes. Havendo dados passados suficientes, o usuário pode optar por realizar uma reidentificação em malha fechada do modelo pela decomposição QR descrita em (4.2) e utilizando a equação (4.4)

\subsubsection{SPC para processo sem ação direta e com horizontes independentes}

Nesta seção, com base nos trabalhos de Huang e Kadali (2008) e Kadali; Huang e Rossiter (2003), serão realizadas duas melhorias no controlador SPC descrito anteriormente. A primeira delas consiste em alterar o modelo de predição em subespaços de forma a se considerar um sistema sem ação direta da entrada na saída. A segunda melhoria refere-se à flexibilização dos horizontes de controle e de 
predição do controlador, transformando-os em parâmetros de sintonia, como ocorre no MPC. Os autores mencionados acima propuseram essas alterações somente para o caso em que o sistema alcança o estado estacionário no último instante do horizonte de controle, ou seja, $u(k+n c+i)=0$ para $i \geq 0$. Essa consideração não será necessária no desenvolvimento realizado a seguir.

\subsubsection{Modelo de predição para processo sem ação direta}

O método SPC apresentado na seção 4.2.1 é projetado para o controle de processos com ação direta da entrada na saída (sistemas com a matriz $D$ do modelo em espaço de estados não nula). Entretanto, na prática, processos da indústria química não apresentam essa característica. Nesses sistemas, o primeiro componente do vetor de saídas futuras $(y(k+1))$ não depende das ações de controle futuras e, portanto, pode ser calculado somente com as informações do passado (até o instante $k$ ). Em outras palavras, para processos reais, o primeiro bloco de linhas da matriz $L_{u}$ da equação (4.1) é vazio.

O último dado de medição disponível em um processo controlado pelo SPC corresponde à resposta do sistema devido à última ação de controle $(u(k))$. Para sistemas sem ação direta, esse dado consiste na saída $y(k+1)$. Porém, o modelo de predição do controlador SPC considera que essa saída é dependente da entrada $u(k+1)$ e, portanto, ainda é uma predição futura. Dessa forma, o último dado medido não é utilizado pelo controlador, que demora um período de amostragem para deixar de usar a predição do modelo e considerar a medida real do processo.

Pode-se transladar o limite entre passado e futuro usado no SPC em um período de tempo. Assim, para processos sem ação direta, a última saída medida e a primeira ação de controle a ser calculada passam a ser, respectivamente, $y(k)$ e $u(k)$. Esse é o enfoque utilizado no MPC do capítulo 2. 
Os horizontes referentes a passado e futuro usados no SPC são determinados pelo horizonte do modelo de predição em subespaços. Logo, o primeiro passo no desenvolvimento de um controlador SPC para sistemas sem ação direta é encontrar um modelo de predição que considere como passado as saídas até o instante $k$ e as entradas até 0 instante $k-1$. Partindo-se do modelo de predição na forma incremental da equação (4.12), pode-se dividir a parcela referente ao passado da seguinte forma:

$$
L_{w}^{i n c} \Delta w_{p}=\left[\begin{array}{ll}
L_{w 1}^{i n c} & L_{w 2}^{i n c}
\end{array}\right]\left[\begin{array}{c}
\Delta w_{p}^{a} \\
\Delta u(k)
\end{array}\right]
$$

Substituindo a equação acima na equação do modelo, tem-se:

$$
\hat{y}_{f}=Y_{k}+L_{w 1}^{i n c} \Delta w_{p}^{a}+\left[\begin{array}{ll}
L_{w 2}^{i n c} & L_{u}^{i n c}
\end{array}\right]\left[\begin{array}{c}
\Delta u(k) \\
\Delta u_{f}
\end{array}\right]
$$

Portanto o modelo de predição em subespaços para sistemas sem ação direta pode ser escrito como:

$$
\hat{y}_{f}^{a}=Y_{k}+L_{w}^{a} \Delta w_{p}^{a}+L_{u}^{a} \Delta u_{f}^{a}
$$

sendo:

$$
\begin{aligned}
& \Delta w_{p}^{a}=\left[\begin{array}{c}
\Delta y_{p}^{a} \\
\Delta u_{p}^{a}
\end{array}\right] ; \quad \hat{y}_{f}^{a}=\hat{y}_{f}=\left[\begin{array}{c}
\hat{y}(k+1) \\
\hat{y}(k+2) \\
\vdots \\
\hat{y}(k+M)
\end{array}\right] ; \quad \Delta u_{f}^{a}=\left[\begin{array}{c}
\Delta u(k) \\
\Delta u(k+1) \\
\vdots \\
\Delta u(k+M)
\end{array}\right] ; \\
& \Delta y_{p}^{a}=\Delta y_{p}=\left[\begin{array}{c}
\Delta y(k-M+1) \\
\Delta y(k-M+2) \\
\vdots \\
\Delta y(k)
\end{array}\right] ; \quad \Delta u_{p}^{a}=\left[\begin{array}{c}
\Delta u(k-M+1) \\
\Delta u(k-M+2) \\
\vdots \\
\Delta u(k-1)
\end{array}\right] .
\end{aligned}
$$

As matrizes desse novo modelo podem ser obtidas a partir das matrizes do modelo em (4.12) como:

$$
\begin{aligned}
& L_{w}^{a}=L_{w 1}^{i n c} \\
& L_{u}^{a}=\left[\begin{array}{ll}
L_{w 2}^{i n c} & L_{u}^{i n c}
\end{array}\right]
\end{aligned}
$$


sendo $L_{w 1}^{i n c} \in R^{l . M \times(m .(M-1)+l . M)}$ e $L_{w 2}^{i n c} \in R^{l . M \times m}$.

É importante notar que, no modelo de predição em subespaços para processos sem ação direta, os horizontes de passado e futuro para as entradas não são simétricos. $O$ vetor de entradas passadas tem um horizonte de $M-1$ instantes, enquanto que 0 horizonte das entradas futuras é $M+1$.

\subsubsection{Flexibilização dos horizontes de controle e de predição}

No modelo de predição em subespaços pode-se identificar três importantes horizontes: o horizonte do vetor de saídas futuras, o horizonte do vetor de entradas futuras e o horizonte dos dados passados. A obtenção do modelo através da identificação com dados amostrados do sistema resulta no modelo de predição com um único valor para todos esses horizontes, que é o parâmetro $M$.

$\mathrm{Na}$ visão do SPC, cada horizonte do modelo de predição em subespaços possui uma função. O horizonte do vetor de saídas futuras corresponde ao horizonte de predição do controlador; o horizonte do vetor das entradas futuras refere-se ao horizonte de controle; e o horizonte dos dados passados é utilizado na estimação do estado atual do sistema. Esse último horizonte normalmente precisa ser longo, para que todas as informações acerca da situação atual do sistema estejam contidas no conjunto de dados passados. Por outro lado, é interessante que os horizontes de predição e de controle sejam menores, facilitando a resolução do problema de otimização. Isso é verdade especialmente para o horizonte de controle, pois ele determina o número de variáveis do problema. Se ele for muito elevado, pode-se gerar um esforço computacional alto e/ou uma instabilidade numérica. Por todos esses motivos a idéia de um modelo de predição em subespaços com horizontes independentes é bastante interessante.

Para a obtenção do modelo de predição em subespaços com horizontes independentes, será considerado que o horizonte dos dados passados permanecerá 
inalterado. Logo, esse horizonte é escolhido na determinação do parâmetro $M$ no momento da identificação. O horizonte de controle, denominado $n c$, e o horizonte de predição, denominado $n p$, podem ser escolhidos pelo usuário com a única restrição de que eles devem ser menores ou iguais a $M$. Uma vez escolhidos os valores de $n c$ e $n p$, o novo modelo de predição em subespaços é obtido através do truncamento das matrizes do modelo original. Partindo-se do modelo na forma incremental para sistemas sem ação direta (4.20), o novo modelo pode ser escrito como:

$$
\hat{y}_{f}^{a h}=Y_{k}+L_{w}^{a h} \Delta w_{p}^{a h}+L_{u}^{a h} \Delta u_{f}^{a h}
$$

sendo:

$$
\begin{aligned}
& \Delta w_{p}^{a h}=\left[\begin{array}{c}
\Delta y_{p}^{a h} \\
\Delta u_{p}^{a h}
\end{array}\right] ; \quad \hat{y}_{f}^{a h}=\left[\begin{array}{c}
\hat{y}(k+1) \\
\hat{y}(k+2) \\
\vdots \\
\hat{y}(k+n p)
\end{array}\right] ; \quad \Delta u_{f}^{a h}=\left[\begin{array}{c}
\Delta u(k) \\
\Delta u(k+1) \\
\vdots \\
\Delta u(k+n c-1)
\end{array}\right] ; \\
& \Delta y_{p}^{a h}=\Delta y_{p}^{a}=\left[\begin{array}{c}
\Delta y(k-M+1) \\
\Delta y(k-M+2) \\
\vdots \\
\Delta y(k)
\end{array}\right] ; \quad \Delta u_{p}^{a h}=\Delta u_{p}^{a}=\left[\begin{array}{c}
\Delta u(k-M+1) \\
\Delta u(k-M+2) \\
\vdots \\
\Delta u(k-1)
\end{array}\right] .
\end{aligned}
$$

Utilizando a notação do Matlab, as matrizes desse modelo podem ser obtidas como:

$$
\begin{aligned}
& L_{w}^{a h}=L_{w}^{a}(1: n p \cdot l,:) \\
& L_{u}^{a h}=L_{u}^{a}(1: n p \cdot l, 1: n c \cdot m)
\end{aligned}
$$

\subsubsection{Algoritmo do controlador SPC com melhorias}

Utilizando o modelo definido em (4.23), é possível propor um algoritmo SPC que incorpore as características desse modelo, ou seja, considere um sistema sem ação direta da entrada na saída e permita utilizar horizontes de controle e de predição distintos. Essas considerações são usadas no método MPC (capítulo 2). Logo, o 
problema de otimização do controlador SPC com melhorias é o mesmo problema definido em (2.7)-(2.9), que é resolvido pelo MPC:

$$
\min _{\Delta u_{f}^{a h}} J_{k}=\sum_{j=1}^{n p}\left(y(k+j \mid k)-y^{S P}\right)^{T} Q\left(y(k+j \mid k)-y^{S P}\right)+\sum_{j=0}^{n c-1} \Delta u(k+j \mid k)^{T} R \Delta u(k+j \mid k)
$$

sujeito a:

$$
\begin{aligned}
& -\Delta u_{\max } \leq \Delta u(k+j \mid k) \leq \Delta u_{\max }, \quad j=0, \ldots, n c-1 \\
& u_{\min } \leq u(k+j \mid k) \leq u_{\max }, \quad j=0, \ldots, n c-1
\end{aligned}
$$

A função objetivo (4.26) pode então ser escrita em função das matrizes e vetores do modelo de predição em subespaços (4.23):

$$
J_{k}=\left(Y_{k}+L_{w}^{a h} \Delta w_{p}^{a h}+L_{u}^{a h} \Delta u_{f}^{a h}-Y_{s p}\right)^{T} \bar{Q}\left(Y_{k}+L_{w}^{a h} \Delta w_{p}^{a h}+L_{u}^{a h} \Delta u_{f}^{a h}-Y_{s p}\right)+\Delta u_{f}^{a h^{T}} \bar{R} \Delta u_{f}^{a h}
$$

Da mesma maneira demonstrada para o MPC, as entradas futuras podem ser escritas em função de suas variações a cada instante por:

$$
u_{f}^{a h}=\bar{I} u(k-1)+\bar{M} \Delta u_{f}^{a h}
$$

Finalmente, o problema de otimização apresentado em (4.26)-(4.28) pode ser escrito na forma quadrática:

$$
\min _{\Delta u_{f}^{a h}} J_{k}=\Delta u_{f}^{a h^{T}} H_{3} \Delta u_{f}^{a h}+2 c_{f 3}{ }^{T} \Delta u_{f}^{a h}+c_{3}
$$

sujeito a: $A_{\text {ineq } 3} \Delta u_{f}^{a h} \leq b_{\text {ineq } 3}$

sendo:

$$
\begin{aligned}
& H_{3}=L_{u}^{a h^{T}} \bar{Q} L_{u}^{a h}+\bar{R} \text {; } \\
& c_{f 3}{ }^{T}=\left[Y_{k}+L_{w}^{a h} \Delta w_{p}^{a h}-Y_{s p}\right]^{T} \bar{Q} L_{u}^{a h} ; \\
& c_{3}=\left[Y_{k}+L_{w}^{a h} \Delta w_{p}^{a h}-Y_{s p}\right]^{T} \bar{Q}\left[Y_{k}+L_{w}^{a h} \Delta w_{p}^{a h}-Y_{s p}\right] \text {; }
\end{aligned}
$$

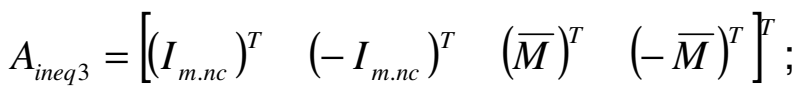




$$
b_{\text {ineq } 3}=\left[\begin{array}{lll}
\left(\bar{I} \Delta u_{\max }\right)^{T} & \left(-\bar{I} \Delta u_{\max }\right)^{T} \quad\left(\bar{I}\left(u_{\max }-u(k-1)\right)\right)^{T} \quad\left(\bar{I}\left(u(k-1)-u_{\min }\right)\right)^{T}
\end{array}\right]^{T} .
$$

As matrizes $\bar{Q}, \bar{R}, Y_{s p}, \bar{M}$ e $\bar{I}$ são definidas pelas mesmas equações usadas no MPC.

\subsection{SIMULAÇÕES E ANÁLISES}

O SPC foi testado para o sistema da coluna debutanizadora da refinaria RPBC, apresentado na seção 3.3.1. Diversas simulações foram realizadas no intuito de analisar detalhadamente as características desse controlador. Por considerar condições mais aderentes às necessidades práticas, optou-se por utilizar o controlador SPC na forma incremental para sistemas sem ação direta e com horizontes independentes.

Os resultados obtidos nas simulações foram divididos em três partes. Na primeira parte é analisado como o parâmetro $M$ influência o desempenho do controlador SPC. Posteriormente, o comportamento do SPC e do MPC são comparados para diferentes situações de controle. Por fim, simula-se o SPC com reidentificação do modelo em malha fechada.

\subsubsection{Influência do horizonte de dados passados no controlador}

A fim de analisar a influência do horizonte de dados passados $M$ no desempenho do controlador, diversas simulações foram realizadas, nas quais o valor de $M$ foi alterado enquanto todos os outros parâmetros foram mantidos constantes, em uma sintonia base. Essa sintonia, que foi definida através de testes por meio de tentativa e erro, é apresentada na Tabela 4.1. Cada teste simulou a situação em que o sistema deve ser controlado em $y^{s p}=\left[\begin{array}{ll}1 & -1\end{array}\right]^{T}$, partindo-se da origem. Logo, como 
condição inicial no modelo do controlador utilizou-se um vetor de dados passados nulo. Em algumas simulações, utilizou-se valores de $M$ menores que os horizontes de predição $(n p)$ e de controle $(n c)$ definidos na sintonia base. Como o limite superior de $n p$ e $n c$ é o parâmetro $M$, nos casos em que $M \leq 7$, considerou-se $n c=M$ e, nos casos em que $M \leq 20$, considerou-se $n p=M$.

Tabela 4.1 - Sintonia base do controlador SPC.

\begin{tabular}{lcc}
\hline \multicolumn{1}{c}{ Parâmetro de Sintonia } & Símbolo & Valor \\
\hline Matriz de pesos das saídas & $Q$ & $\operatorname{diag}\left(\left[\begin{array}{ll}1 & 1\end{array}\right]\right)$ \\
\hline Matriz de pesos das entradas & $R$ & $\operatorname{diag}\left(\left[\begin{array}{ll}0.1 & 0.1\end{array}\right]\right)$ \\
\hline Limite máximo das entradas & $u_{\max }$ & {$\left[\begin{array}{ll}15 & 15\end{array}\right]^{T}$} \\
\hline Limite mínimo das entradas & $u_{\min }$ & {$\left[\begin{array}{cc}-10 & -10\end{array}\right]^{T}$} \\
\hline Variação máxima das entradas & $\Delta u_{\max }$ & {$\left[\begin{array}{ll}1 & 1\end{array}\right]^{T}$} \\
\hline Horizonte de predição & $n p$ & 20 \\
\hline Horizonte de controle & $n c$ & 7 \\
\hline Horizonte de dados passados & $M$ & 70 \\
\hline
\end{tabular}

O parâmetro $M$ foi analisado para três casos distintos. O primeiro consiste na situação hipotética em que o modelo utilizado no controlador é perfeito. Nesse teste assumiu-se que a planta e o modelo do SPC são representados pelo Modelo C1. $\mathrm{O}$ segundo representa uma situação mais real, na qual o sistema precisa ser controlado em uma condição de operação diferente daquela usada na identificação do modelo. Para esse caso, considerou-se que o Modelo C1 é usado no controlador e a planta é representada pelo Modelo C2. Por fim, o terceiro caso é uma extensão do primeiro: a condição de operação no controle e na identificação do modelo é a mesma (representada pelo Modelo C1), porém os dados usados na identificação foram coletados na presença de ruído.

Em todos os testes o modelo de predição em subespaços usado no SPC foi obtido por meio de identificação. Para o primeiro e segundo casos foram utilizados os dados do experimento $A$ (Tabela 3.4 ), enquanto que os dados do experimento $D$ (Tabela 3.4) foram utilizados no terceiro caso. Um resumo dos testes realizados é apresentado na Tabela 4.2. 
Tabela 4.2 - Resumo dos estudos realizados para análise do parâmetro $M$ no SPC.

\begin{tabular}{cccc}
\hline Estudo & $\begin{array}{c}\text { Representação } \\
\text { do Sistema }\end{array}$ & $\begin{array}{c}\text { Modelo do } \\
\text { Controlador }\end{array}$ & Identificação do Modelo \\
\hline Caso 1 & Modelo C1 & Modelo C1 & Dados na ausência de ruído \\
\hline Caso 2 & Modelo C2 & Modelo C1 & Dados na ausência de ruído \\
\hline Caso 3 & Modelo C1 & Modelo C1 & Dados com ruído de variância 0.001 \\
\hline
\end{tabular}

A Figura 4.5 ilustra o resultado obtido nas simulações realizadas para o caso do controlador SPC com modelo perfeito (Caso 1). Observa-se que o desempenho do controlador está diretamente ligado à qualidade do modelo de predição em subespaços obtido na identificação. A qualidade do modelo foi analisada anteriormente através de comparações das respostas ao degrau (Figura 4.2). Por exemplo, para $M=3$, a identificação gerou um modelo de pior qualidade, e conseqüentemente o controlador SPC se desempenhou de maneira menos satisfatória. Além disso, nota-se, na Figura 4.5, que a partir de determinado valor o parâmetro $M$ apresenta pouca influência no comportamento do SPC, sendo que para $M=50$ e $M=100$ os resultados obtidos foram idênticos.
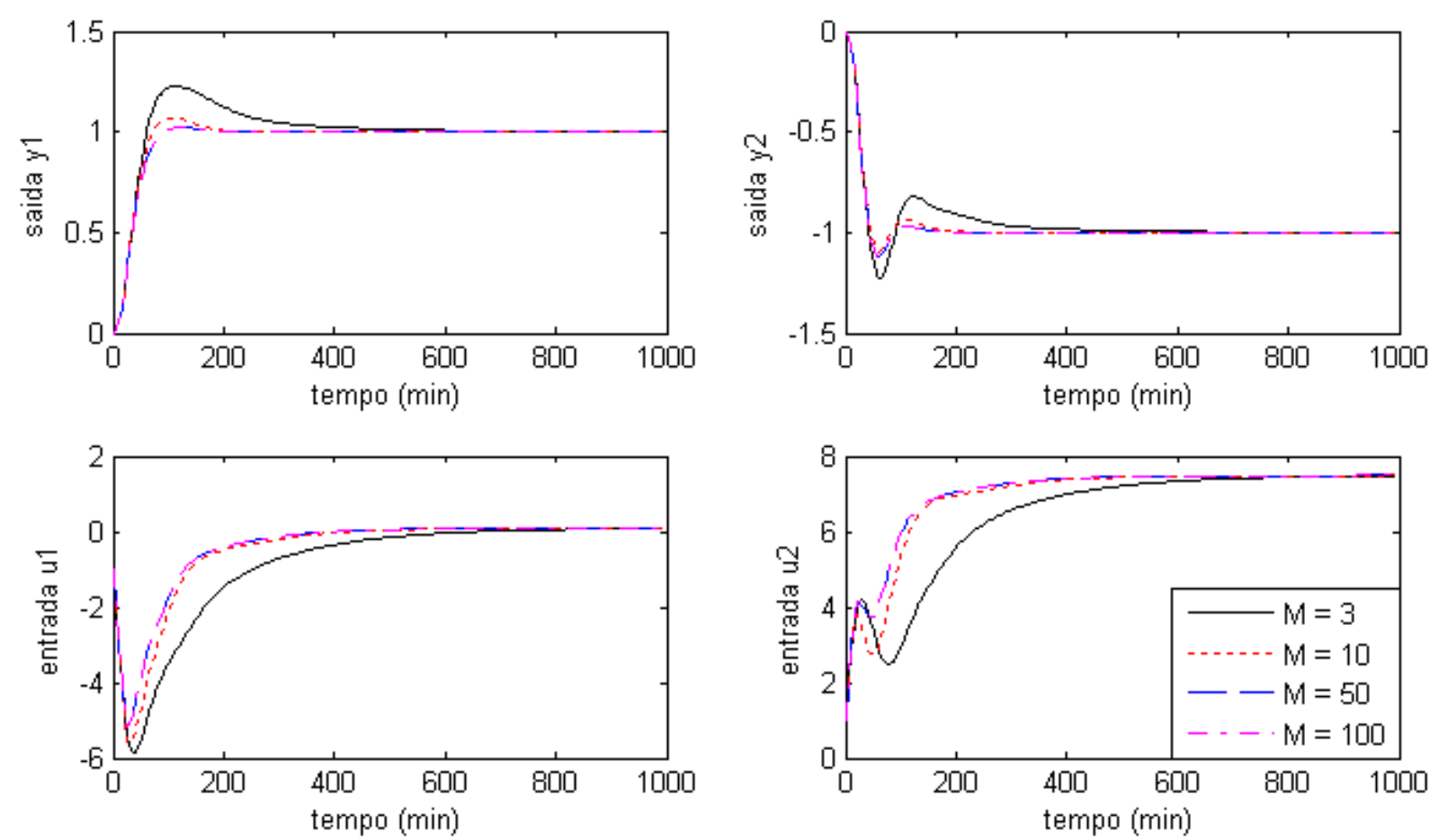

Figura 4.5 - Análise da influência de $M$ no SPC para o caso de controlador com modelo perfeito.

O resultado das simulações para o caso de erro no modelo do controlador devido à mudança de ponto de operação (Caso 2) está mostrado na Figura 4.6. Nota-se que o comportamento do SPC foi bem diferente daquele observado no primeiro caso. É 
possível verificar que, para uma larga faixa de valores, o parâmetro $M$ exerce grande influência no desempenho do controlador. Observa-se ainda que valores muito baixos de $M$ podem causar oscilações de amplitudes elevadas tanto nas variáveis controladas como nas variáveis manipuladas.
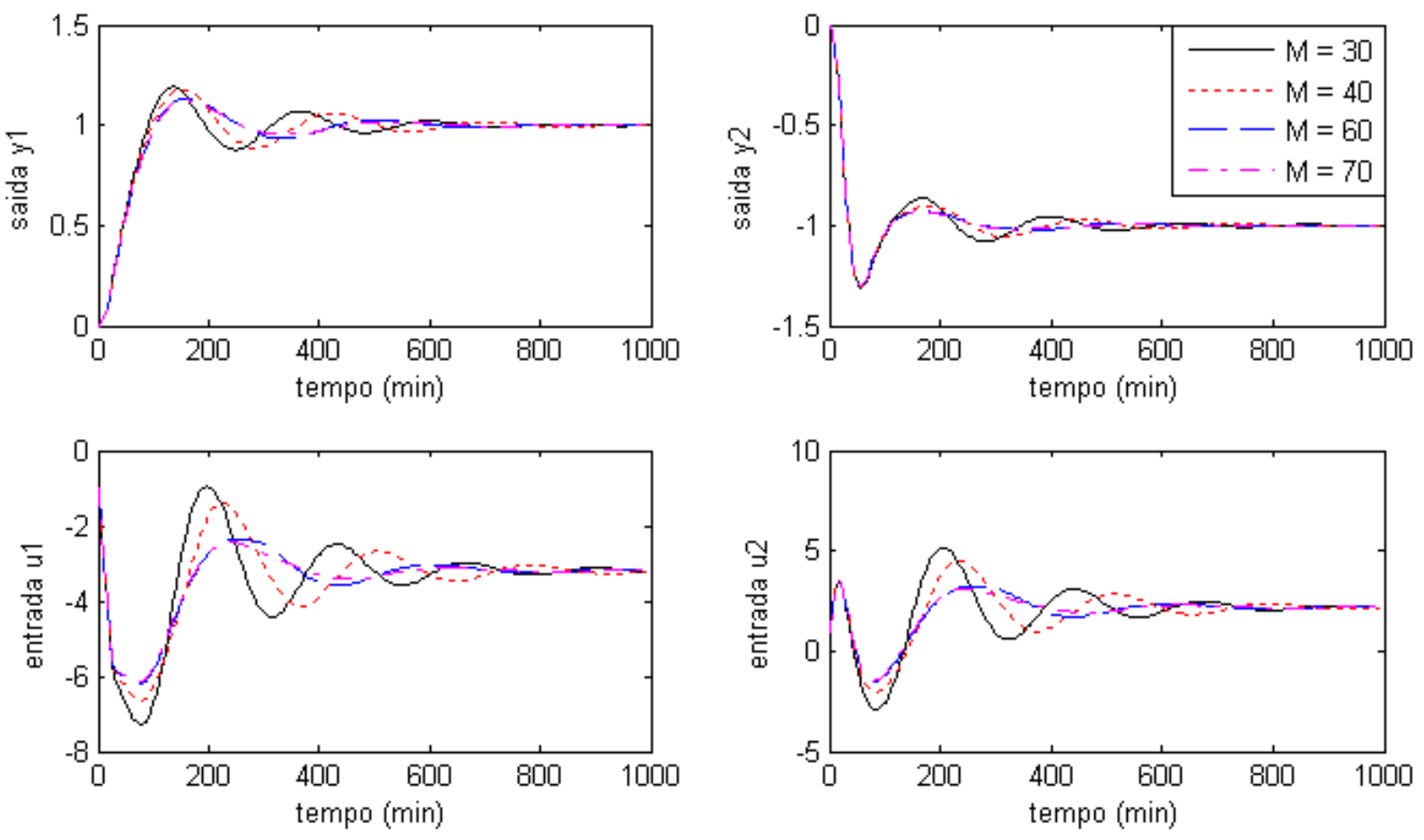

Figura 4.6 - Análise da influência de $M$ no SPC para o caso de controlador com erro no modelo devido à mudança no ponto de operação.

Finalmente a Figura 4.7 apresenta o resultado obtido para o Caso 3, no qual os dados usados na identificação do modelo foram coletados na presença de ruído. Esse resultado se assemelha ao resultado obtido para o Caso 1. Entretanto, observa-se que, para valores de $M$ muito elevados, a adição do ruído nos dados de identificação gerou certo decaimento no desempenho do SPC. Esse comportamento é devido a problemas de sobre-parametrização do modelo de predição em subespaços.

Em situações práticas, o modelo do controlador nunca é uma representação perfeita do sistema real, principalmente porque o sistema, que normalmente é não-linear, é representado por um modelo linear. Além disso, os dados sempre são medidos na presença de ruídos. Nesses casos, um horizonte $M$ grande introduz grandes erros de predição (WANG; HUANG; CHEN, 2007). Logo, o parâmetro $M$ deve ser cuidadosamente escolhido e consiste em uma decisão do projeto do controlador. 

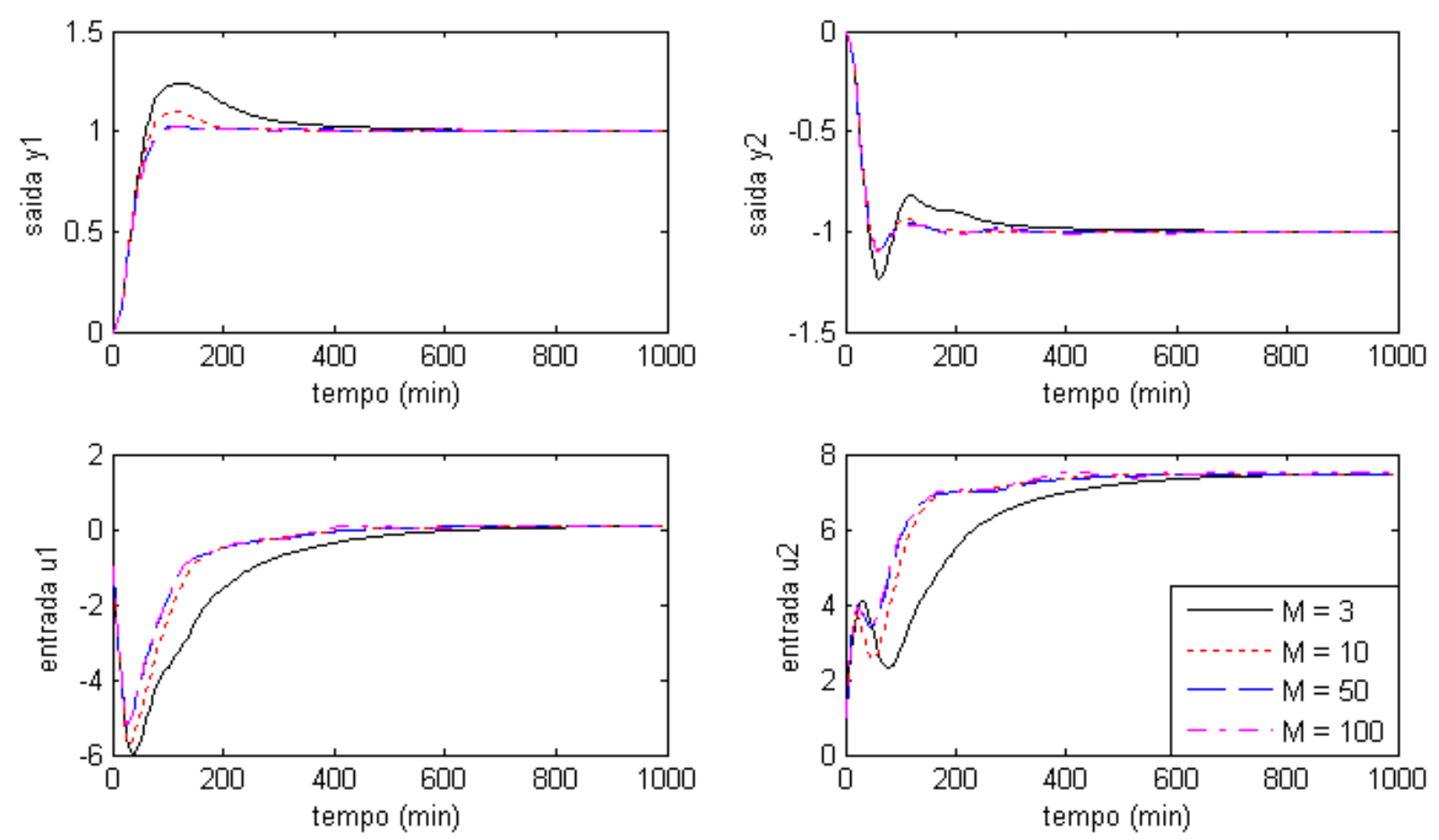

Figura 4.7 - Análise da influência de $M$ no SPC para o caso de controlador com erro no modelo devido à identificação com dados com ruído.

\subsubsection{Comparação entre SPC e MPC}

Por meio de simulações com o sistema da coluna debutanizadora, os desempenhos dos controladores SPC e MPC foram comparados para diversas situações práticas de controle de processos. Em cada simulação o sistema foi controlado em $y^{s p}=\left[\begin{array}{ll}1 & -1\end{array}\right]^{T}$, partindo-se da origem. Para tornar a comparação mais coerente, a mesma sintonia foi utilizada para ambos os controladores. Optou-se por manter a sintonia usada na seção anterior, que foi apresentada na Tabela 4.1.

Alguns estudos foram realizados para o caso de controladores com modelo perfeito, considerando a planta e o modelo dos controladores SPC e MPC representados pelo Modelo C1. Em outros estudos foram utilizados controladores com erro no modelo, sendo que os modelos dos controladores e a planta foram representados pelo Modelo C1 e Modelo C2, respectivamente. Em todos os testes, o modelo de predição em subespaços usado no SPC foi obtido pela identificação com os dados do experimento $A$ (Tabela 3.4). O modelo do MPC foi obtido pela conversão da 
função de transferência do Modelo C1 para o modelo em espaço de estados na forma incremental. A seguir estão descritos os estudos realizados e os resultados obtidos. Uma análise detalhada é realizada para cada caso.

Estudo 1: Controladores com modelos perfeitos e processo sem ruído.

O SPC e o MPC foram simulados para o caso hipotético dos modelos dos controladores serem perfeitos e o processo não ser afetado por nenhum ruído. 0 resultado pode ser visto na Figura 4.8.
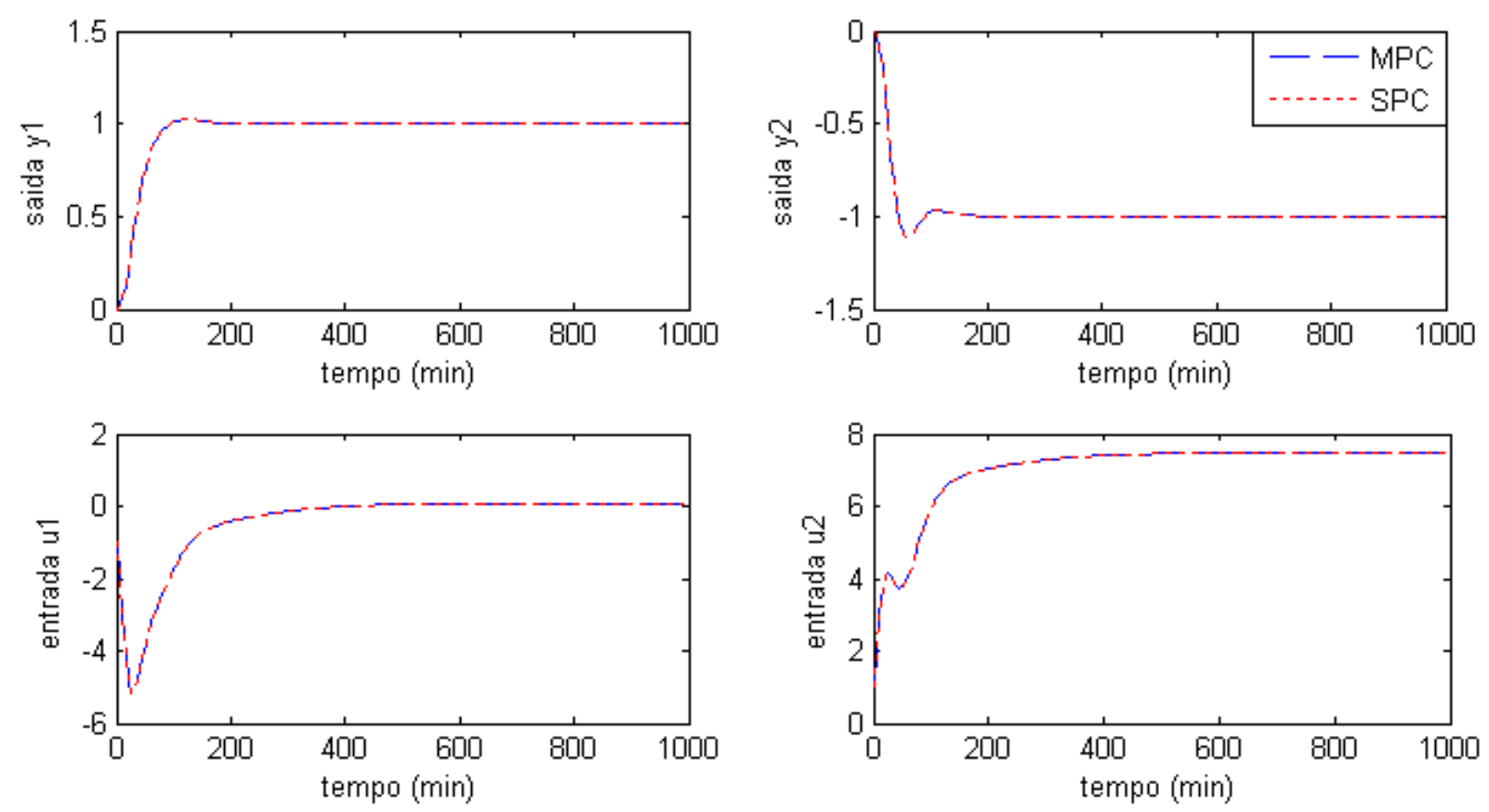

Figura 4.8 - Comparação entre SPC e MPC. Caso de controladores com modelos perfeitos e processo sem ruído.

Observa-se que nessa simulação ambos os controladores apresentam comportamento idêntico, comprovando a equivalência desses dois métodos na situação ideal de controle.

Estudo 2: Controladores com modelos perfeitos e distúrbio pontual na entrada.

Para o SPC e o MPC sem erro nos modelos foi simulado o caso em que no instante 500 minutos um distúrbio de intensidade $\left[\begin{array}{ll}-2 & 2\end{array}\right]^{T}$ é adicionado na entrada do processo. O resultado está mostrado na Figura 4.9. O desempenho dos dois 
controladores foi idêntico. Como se considerou que a entrada do sistema é medida, o distúrbio também é indiretamente medido e conseqüentemente é usado nos modelos do SPC e do MPC para o cálculo das predições futuras. No modelo do SPC esse dado é usado diretamente na predição, enquanto que no modelo do MPC ele é usado no cálculo intermediário do estado do sistema. Somando-se a isso, há o fato de que ambos os modelos são perfeitos e por isso realizam a predição correta das saídas futuras, de modo que os dois problemas de otimização encontram as mesmas ações ótimas de controle.
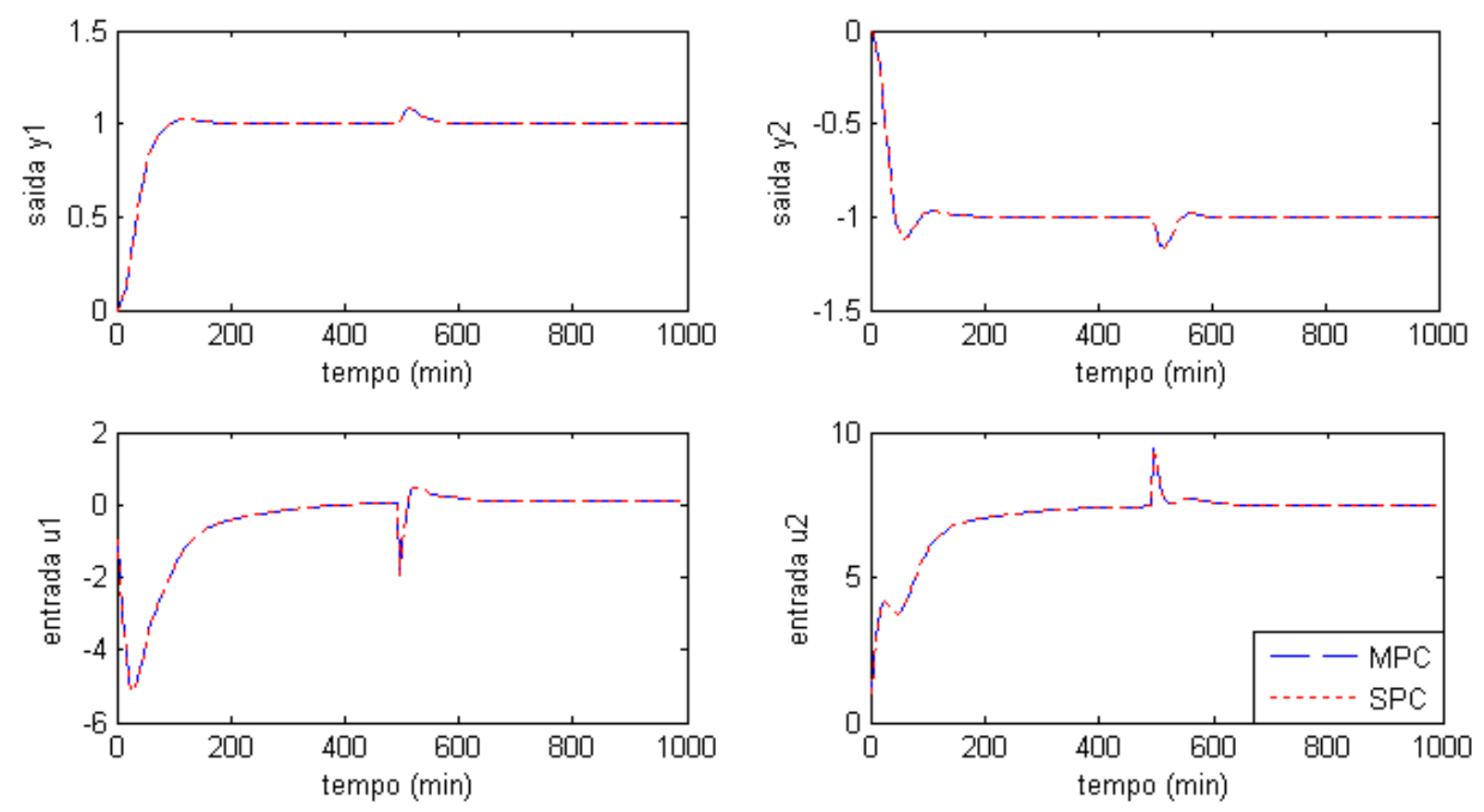

Figura 4.9 - Comparação entre SPC e MPC. Caso de controladores com modelos perfeitos e distúrbio pontual na entrada do processo.

Estudo 3: Controladores com modelos perfeitos e distúrbio pontual na saída.

Para o SPC e o MPC sem erro nos modelos foi simulado o caso em que no instante 500 minutos um distúrbio de intensidade $\left[\begin{array}{ll}-2 & 2\end{array}\right]^{T}$ é adicionado na saída do processo. O resultado está mostrado na Figura 4.10. Nesse caso, nota-se que o SPC obteve desempenho melhor. O modelo de predição em subespaços desse controlador utiliza a saída no instante atual diretamente no cálculo da predição das saídas futuras. Por outro lado, o modelo em espaço de estados do MPC utiliza o estado atual para realizar as predições futuras, sendo que no cálculo desse estado, a saída medida é filtrada pelo observador de estados. Portanto, o MPC não 
considera o distúrbio em sua totalidade, agindo de uma maneira mais suave e conseqüentemente, mais demorada.
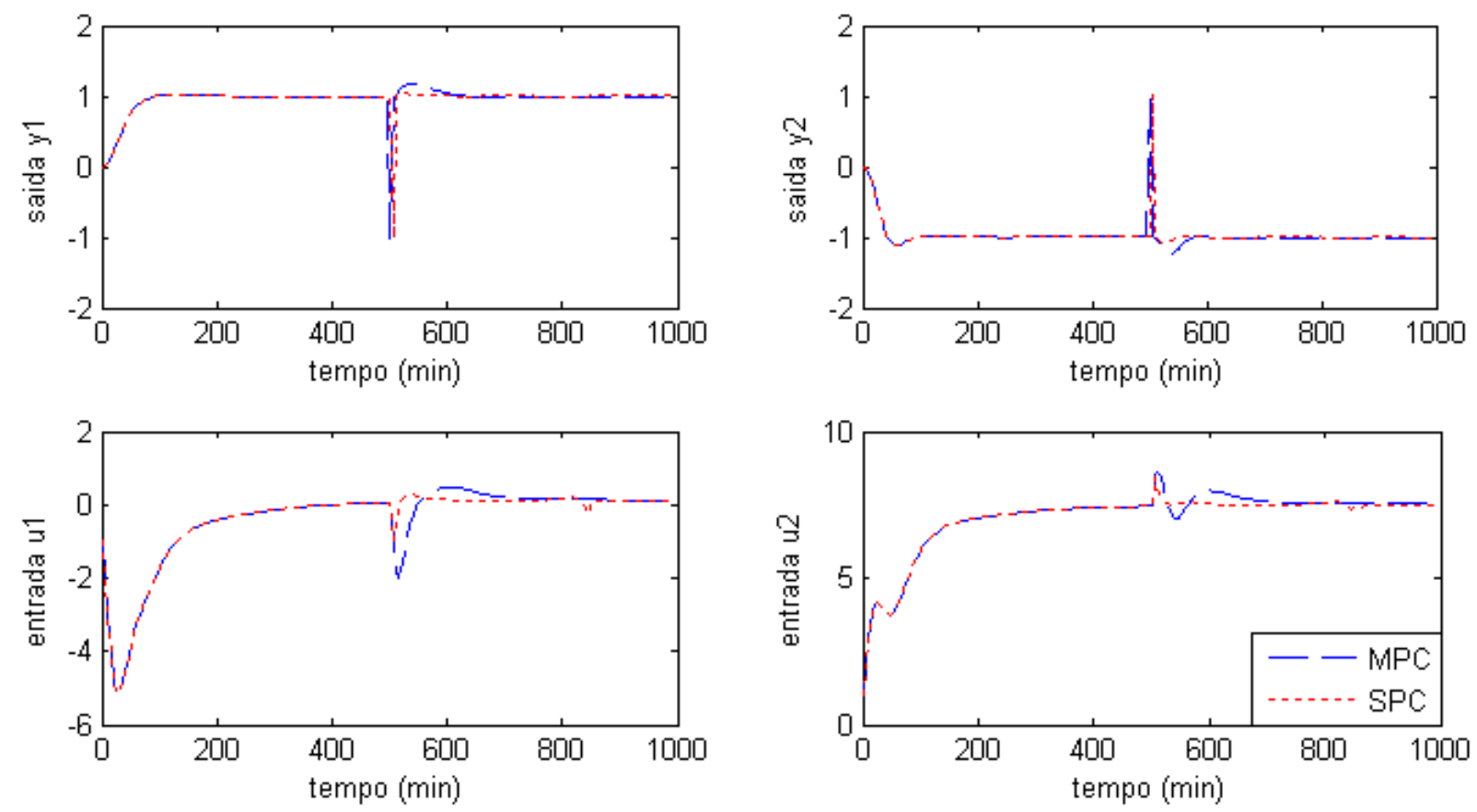

Figura 4.10 - Comparação entre SPC e MPC. Caso de controladores com modelos perfeitos e distúrbio pontual na saída do processo.

Estudo 4: Controladores com modelos perfeitos e ruído aleatório na entrada.
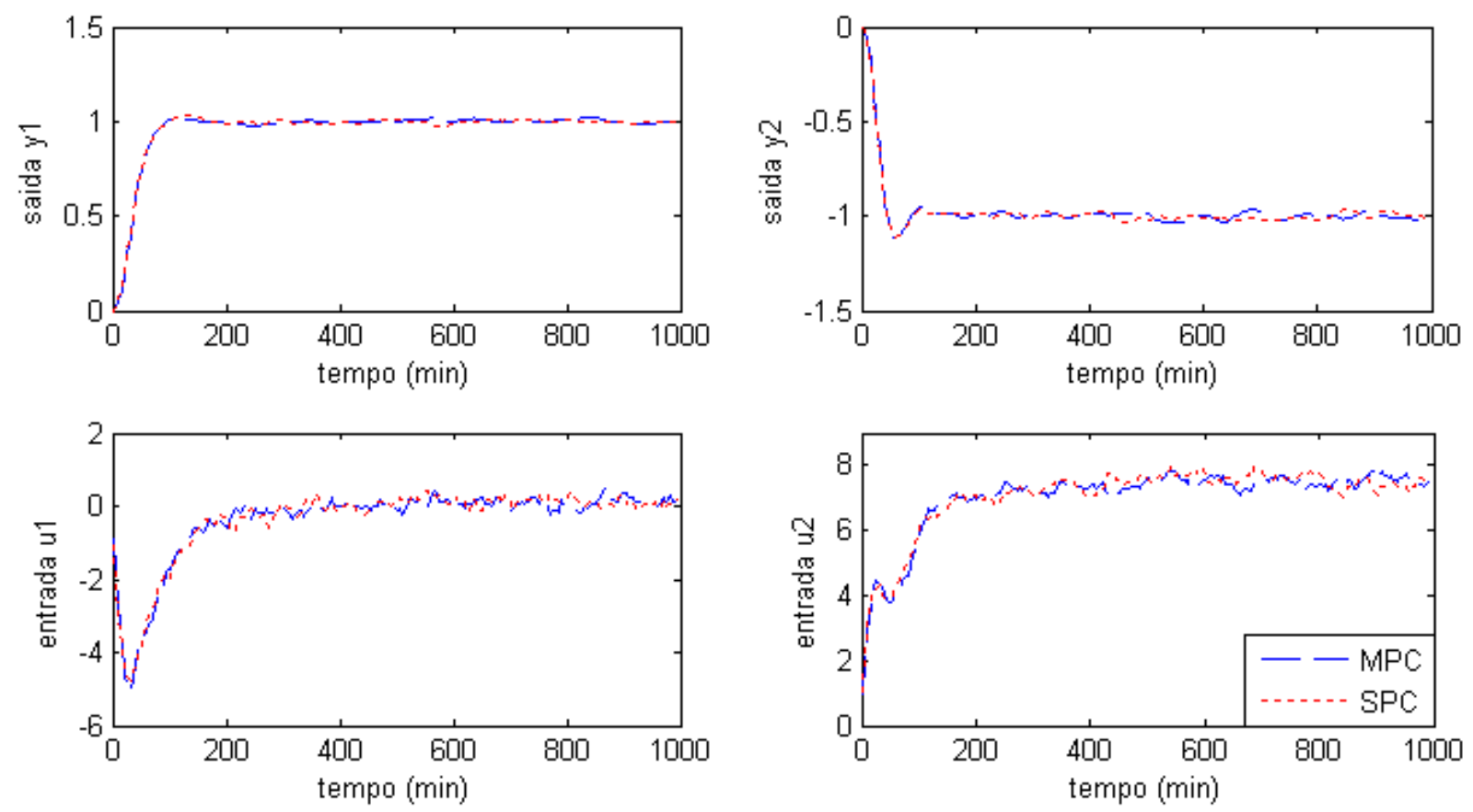

Figura 4.11 - Comparação entre SPC e MPC. Caso de controladores com modelos perfeitos e ruído aleatório de variância 0.01 na entrada do processo. 
Nessa simulação, foi aplicado um ruído randômico de variância 0.01 diretamente nas entradas do processo. A Figura 4.11 mostra o resultado. Verifica-se que o desempenho dos controladores foi muito próximo, mantendo-se a coerência com o resultado obtido para o caso de distúrbio pontual na entrada do processo.

Estudo 5: Controladores com modelos perfeitos e ruído aleatório na saída.

Nessa simulação, foi aplicado um ruído randômico de variância 0.01 diretamente nas saídas do processo. A Figura 4.12 mostra o resultado. Pode-se dizer que o desempenho do MPC foi superior em relação ao desempenho do SPC. O comportamento das variáveis controladas foi similar para ambos os controladores, visto que o ruído aleatório foi adicionado diretamente nas saídas e, portanto, uma diminuição das oscilações não está ao alcance dos controladores. Porém, as mudanças nas entradas do processo controlado pelo MPC foram mais suáveis. Isso se deve exatamente ao fato de que esse controlador não utiliza a saída medida diretamente em seu modelo.
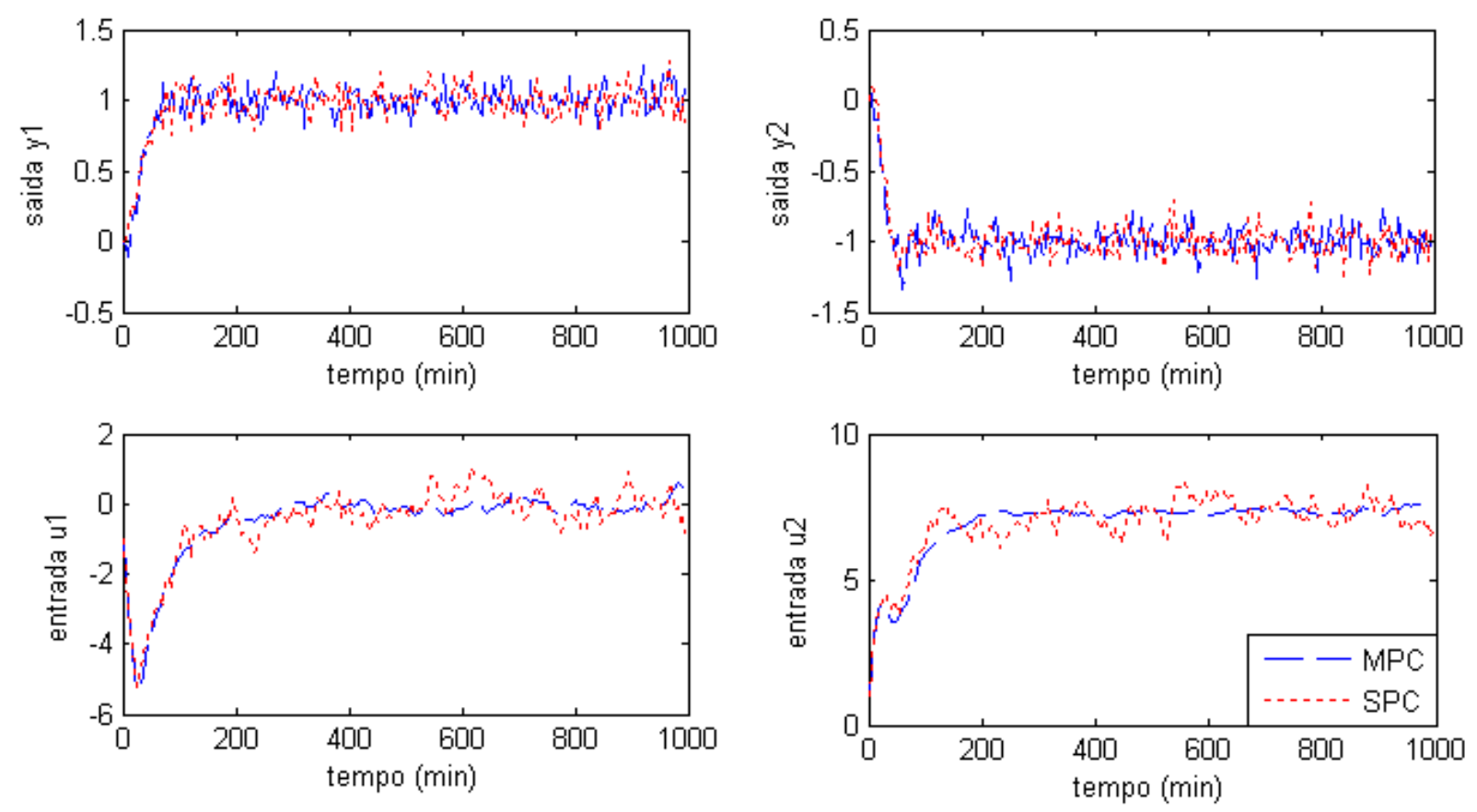

Figura 4.12 - Comparação entre SPC e MPC. Caso de controladores com modelos perfeitos e ruído aleatório de variância 0.01 na saída do processo.

Por utilizar as medições da planta diretamente para fazer as predições do futuro, 0 SPC é muito suscetível a ruídos nas saídas, como erros de medição. Por outro lado, 
o modelo usado no MPC normalmente não possui seus estados medidos, sendo necessário o uso de um observador de estados, como o Filtro de Kalman. O Filtro de Kalman tem a função de realizar um pré-tratamento no erro de predição antes de adicioná-lo ao modelo. Esse filtro considera que há incertezas na predição e também na medida da saída e, portanto, suaviza a correção do estado.

Estudo 6: Controladores com erro nos modelos e processo sem ruído.

Foi considerada a situação em que a planta deve ser controlada em um ponto de operação diferente daquele em que foi realizada a identificação dos modelos dos controladores. O resultado obtido é apresentado na Figura 4.13. O controlador SPC não teve um desempenho tão bom quanto o MPC, oscilando um pouco no início da simulação. Porém ele conseguiu levar as variáveis controladas a seus respectivos set-points em um intervalo de tempo razoável. O MPC levou o processo ao ponto desejado de uma forma mais suave, fato que se deve ao uso do Filtro de Kalman. Por fim, através dessa simulação e de outros testes realizados ao longo deste trabalho, percebe-se que o SPC é mais dependente da qualidade do modelo utilizado do que o controlador MPC.
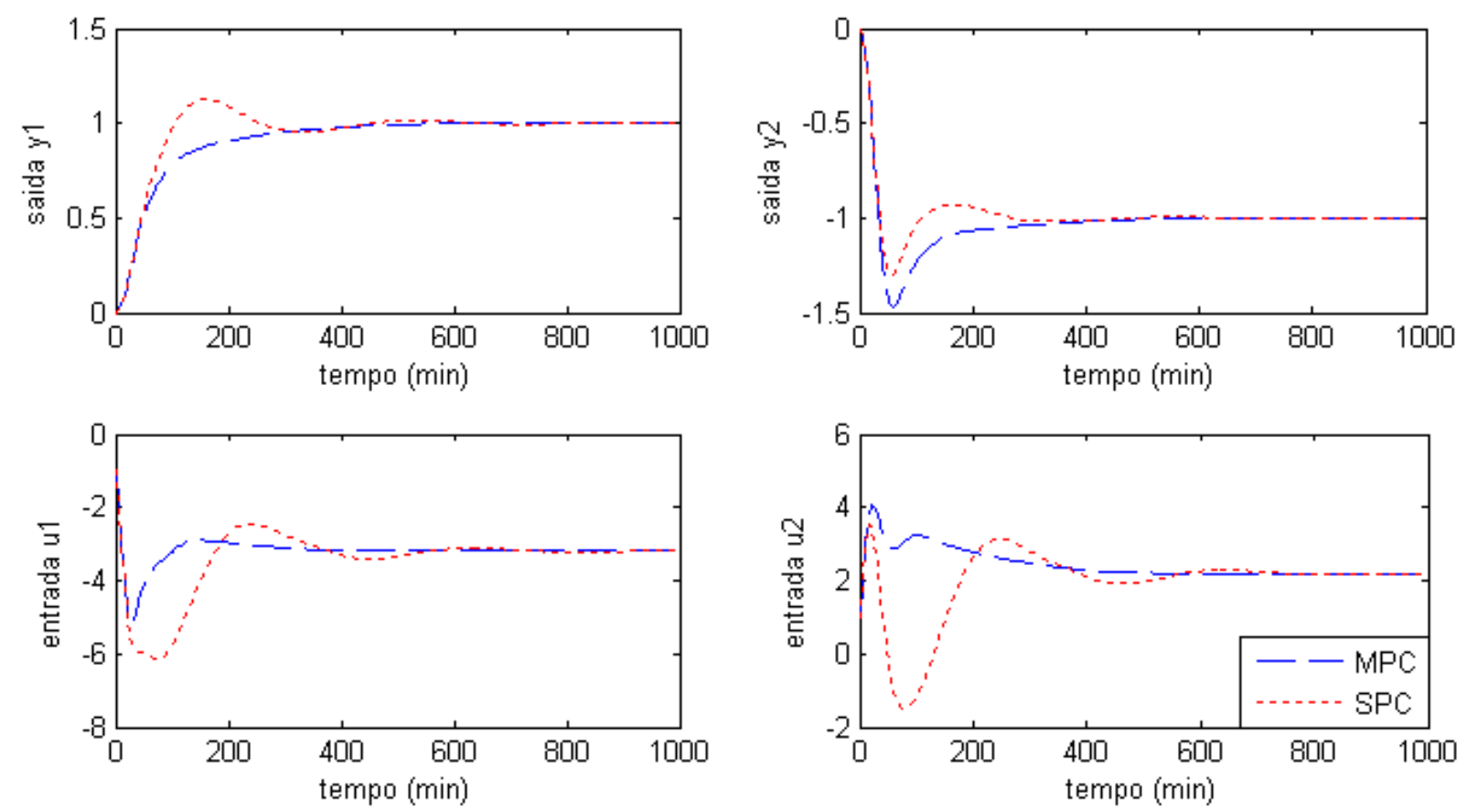

Figura 4.13 - Comparação entre SPC e MPC. Caso de controladores com erro nos modelos e processo sem ruído. 


\subsubsection{Análise do SPC com reidentifação em malha fechada}

Para testar o efeito da reidentificação do modelo de predição em subespaços, realizou-se uma simulação na qual o MPC foi comparado ao SPC com reidentificação em malha fechada. Utilizou-se, para ambos os controladores, a mesma sintonia (Tabela 4.1). O processo foi representado pelo Modelo C2 enquanto os modelos dos controladores foram representados pelo Modelo C1. Os modelos dos controladores foram obtidos pelo mesmo procedimento usado na seção 4.3.2.

A simulação consistiu no controle do processo em $y^{s p}=\left[\begin{array}{ll}1 & -1\end{array}\right]^{T}$, partindo-se da origem. No instante 2500 minutos foi adicionado um distúrbio pontual na entrada de intensidade $\left[\begin{array}{ll}-5 & 7\end{array}\right]^{T}$. Após 4000 minutos do início da simulação, o modelo de predição em subespaços do SPC foi reidentificado em malha fechada, enquanto 0 modelo do MPC permaneceu constante. Finalmente, no instante 4250 minutos, outro distúrbio pontual foi adicionado à entrada do processo, desta vez com intensidade de $\left[\begin{array}{ll}6 & -5\end{array}\right]^{T}$. O resultado está mostrado na Figura 4.14.

Esperava-se que, após a reidentificação do modelo de predição usado no controlador SPC, esse superasse o desempenho do MPC, visto que o SPC teria o modelo corrigido e o MPC continuaria utilizando um modelo correspondente a outro ponto de operação. Porém, na Figura 4.14, observa-se que o desempenho do SPC é constante ao longo da simulação, sendo um pouco pior que o desempenho do MPC, mesmo após a reidentificação. Isso se deve ao fato de que os dados amostrados e usados na identificação não eram persistentemente excitantes. Portanto, percebe-se que para se obter um modelo de boa qualidade é extremante importante excitar corretamente a planta durante a etapa de coleta de dados. Esse aspecto será tratado de forma detalhada no próximo capitulo deste trabalho. 

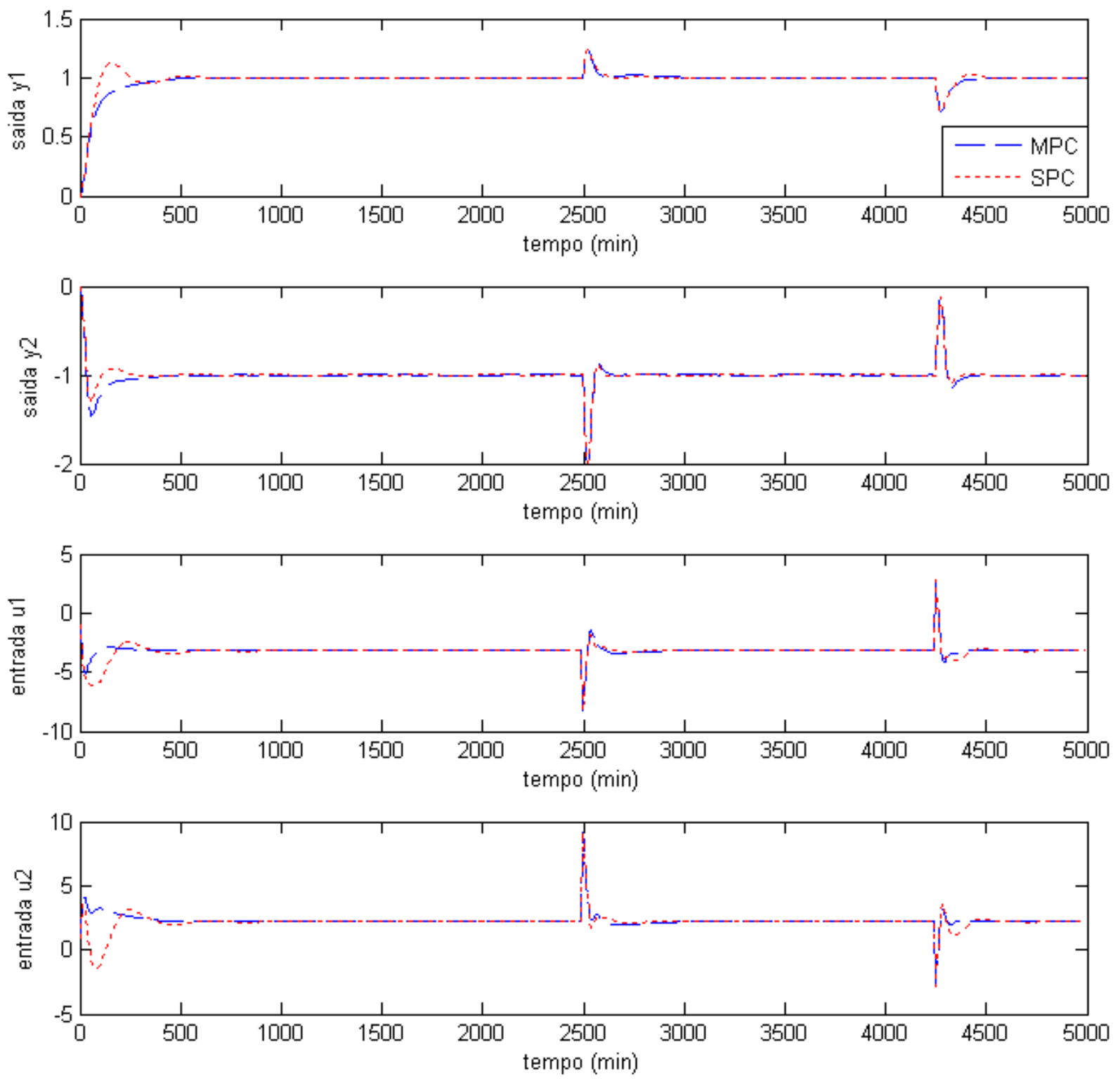

Figura 4.14 - Controlador SPC com reidentificação do modelo sem excitação adicional. 


\section{SPC COM EXCITAÇÃO INTERNA}

No capítulo anterior foi apresentado o conceito do método SPC e foi mostrado como essa técnica de controle pode ser utilizada com reidentificação do modelo em malha fechada. Esse enfoque é bastante interessante quando se deseja reajustar o modelo de sistemas variantes no tempo ou sistemas não-lineares com ponto de operação móvel. Porém, foi comprovado que, se os dados coletados não forem suficientemente informativos, a reidentificação do sistema pode resultar em uma piora na qualidade do modelo e, conseqüentemente, no desempenho do controlador.

Para que a identificação de um modelo seja bem sucedida, é necessário amostrar dados persistentemente excitantes. Por outro lado, deve-se manter a planta dentro de certos limites, por questões econômicas e de segurança. Como forma de garantir que os dados sejam adequadamente excitados ao mesmo tempo em que um controle mínimo do sistema é mantido, surgiram os métodos de controle com excitação interna. Esses métodos consistem em algoritmos de controle preditivo com modificações na função objetivo ou nas restrições do problema de otimização. Exemplos dessa técnica de excitação podem ser encontrados em Shouche et al. (1998); Aggelogiannaki e Sarimveis (2006), e Ballin (2008).

Uma grande desvantagem da maioria dos algoritmos de controle preditivo com excitação interna é que os termos e/ou restrições adicionadas são não-lineares, tornando o problema de otimização não-convexo. Problemas não-convexos, além de serem mais suscetíveis a questões de convergência, apresentam ótimos locais, que podem tornar a solução sub-ótima.

No enfoque do SPC, Hallouzi e Verhaegen (2008) propuseram um controlador preditivo com excitação interna capaz de gerar dados persistentemente excitantes. Nesse método um termo referente à excitação é adicionado na função objetivo, mantendo a convexidade do problema de otimização. 
A seguir serão propostos três algoritmos de excitação interna para o método SPC, com base na idéia apresentada em Hallouzi e Verhaegen (2008). O primeiro algoritmo, apresentado na seção 5.1, consiste em pequenas adaptações do método apresentado por esses autores. Os outros dois algoritmos (seção 5.2 e seção 5.3) apresentam novas alternativas de se adicionar a condição de excitação no problema de otimização. Esses métodos foram testados por meio de simulações no sistema da coluna debutanizadora e os resultados obtidos serão descritos na seção 5.4.

\subsection{CONTROLADOR COM EXCITAÇÃO DIRETA NA ENTRADA}

\subsubsection{Direções menos excitadas}

O modelo de predição em subespaços identificado a partir de dados amostrados é calculado pela decomposição QR de uma matriz de blocos de Hankel, conforme foi apresentado na equação (4.2):

$$
\left[\begin{array}{c}
W_{p} \\
U_{f} \\
Y_{f}
\end{array}\right]=\left[\begin{array}{ccc}
R_{11} & 0 & 0 \\
R_{21} & R_{22} & 0 \\
R_{31} & R_{32} & R_{33}
\end{array}\right]\left[\begin{array}{c}
Q_{1}^{T} \\
Q_{2}{ }^{T} \\
Q_{3}{ }^{T}
\end{array}\right]
$$

A partir dessa decomposição, as matrizes $L_{w}$ e $L_{u}$ do modelo de predição são calculadas pela equação (4.4):

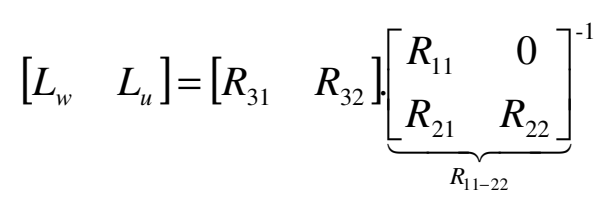

Para que a equação acima possua uma única solução, a matriz $R_{11-22}$ precisa ser invertível e, portanto, não-singular. Logo, a identificabilidade do sistema está diretamente ligada a não-singularidade da matriz de blocos de Hankel definida em 
(4.2). Por esse motivo, o conceito de persistência de excitação usado no controlador SPC baseia-se na geração de dados que tornem essa matriz não-singular.

O termo $\left[\begin{array}{ll}R_{21} & R_{22}\end{array}\right]$ da equação (4.2), refere-se ao conjunto das entradas passadas mais recentes, que, a cada período de amostragem, é atualizado com a inclusão da última ação de controle implementada. Portanto, esse termo consiste na parcela da matriz (4.2) que sofre influência direta da ação do controlador. A idéia do SPC com excitação interna é analisar esse termo e identificar direções nas quais as entradas passadas receberam menor excitação. Essa informação é então utilizada pelo SPC para forçar as próximas ações de controle nessas direções menos excitadas.

O primeiro passo no desenvolvimento do SPC com excitação interna é então entender como as direções menos excitadas podem ser calculadas. Por meio de uma decomposição SVD do termo $\left[\begin{array}{ll}R_{21} & R_{22}\end{array}\right]$, obtém-se:

$$
\left[\begin{array}{ll}
R_{21} & R_{22}
\end{array}\right]=\left[\begin{array}{llll}
c_{1} & c_{2} & \cdots & c_{M \cdot m}
\end{array}\right]\left[\begin{array}{ccccccc}
s_{1} & 0 & \cdots & 0 & 0 & \cdots & 0 \\
0 & s_{2} & \cdots & 0 & 0 & \cdots & 0 \\
\vdots & \vdots & \ddots & \vdots & \vdots & \ddots & \vdots \\
0 & 0 & \cdots & s_{M \cdot m} & 0 & \cdots & 0
\end{array}\right]\left[\begin{array}{c}
v_{1} \\
v_{2} \\
\vdots \\
v_{M . m} \\
\vdots \\
v_{j}
\end{array}\right]
$$

Nessa decomposição, os valores singulares são apresentados em ordem decrescente. A direção menos excitada é aquela referente ao menor valor singular, e, portanto, a direção $c_{M \cdot m}$. A segunda direção menos excitada é a $c_{M \cdot m-1}$, e assim por diante.

Uma decomposição SVD pode ser computacionalmente extensa, se a matriz a ser decomposta for muito grande. Note também que, para o propósito do controlador SPC, são utilizados apenas os últimos componentes dessa decomposição, não sendo necessário o seu cálculo completo. Por esse motivo, Hallouzi e Verhaegen (2008) apresentaram um algoritmo iterativo para o cálculo dos últimos componentes de uma decomposição SVD. Durante o desenvolvimento deste trabalho, os tempos de execução das decomposições SVD foram relativamente baixos e, portanto, não 
houve necessidade do uso desse algoritmo, sendo que a decomposição foi realizada completamente pelo comando svd do Matlab.

\subsubsection{Metodologia do controlador}

O controlador SPC com excitação interna resolve, a cada iteração, o problema de otimização convencional de controle preditivo com a função custo acrescida de um termo referente à persistência de excitação dos dados:

$$
\begin{aligned}
\min _{\Delta u_{f}^{a}} & J_{k}^{e x c}=J_{k}+P J_{k}^{a d i c 1} \\
& \text { sujeito a: } \\
& -\Delta u_{\max } \leq \Delta u(k+j \mid k) \leq \Delta u_{\max }, \quad j=0, \ldots, M-1 \\
& u_{\min } \leq u(k+j \mid k) \leq u_{\max }, \quad j=0, \ldots, M-1
\end{aligned}
$$

sendo $J_{k}$ a função custo do controlador SPC sem excitação:

$$
J_{k}=\sum_{j=1}^{M}\left(y(k+j \mid k)-y^{S P}\right)^{T} Q\left(y(k+j \mid k)-y^{S P}\right)+\sum_{j=0}^{M-1} \Delta u(k+j \mid k)^{T} R \Delta u(k+j \mid k)
$$

O termo adicional $J_{k}^{\text {adicl }}$ tem o objetivo de forçar as ações de controle futuras nas direções menos excitadas. O parâmetro $P$ é um parâmetro de sintonia que determina a importância da excitação em relação ao controle. Considerando apenas $n_{p e}$ direções menos excitadas, calculadas conforme procedimento apresentado na seção anterior, o termo adicional pode ser escrito como:

$$
J_{k}^{\text {adic1 }}=\left\|\rho\left[\begin{array}{c}
c_{M . m} \\
c_{M . m-1} \\
\vdots \\
c_{M . m-n_{p e}+1}
\end{array}\right]-\left[\begin{array}{c}
I_{M . m} \\
I_{M . m} \\
\vdots \\
I_{M . m}
\end{array}\right] u_{f}^{a}\right\|_{2}^{2}
$$


Na equação (5.6), o parâmetro $\rho$ é utilizado para ajustar o nível de excitação. Foi necessário introduzi-lo na função objetivo, pois os valores absolutos das direções menos excitadas podem ser pequenos e, assim, insuficientes para excitar persistentemente o sistema.

Observe que, devido à definição de persistência de excitação utilizada no controlador SPC, o horizonte de controle $n c$ obrigatoriamente deve ser igual à $M$. As direções menos excitadas possuem $M$ blocos de linhas, pois são calculadas a partir da matriz de blocos de Hankel das entradas passadas usadas na identificação do modelo. Logo, para se manter a consistência entre essas direções e o vetor de entradas futuras, esse último também deve conter $M$ blocos de linhas. Assim, o controlador SPC com excitação interna pode ser aplicado para um processo sem ação direta, porém não é possível utilizar horizontes de predição e de controle independentes. Por esse motivo, utiliza-se o modelo definido na seção 4.2.2.1.

Analogamente à equação (4.30), pode-se escrever:

$$
u_{f}^{a}=\bar{I} u(k-1)+\bar{M} \Delta u_{f}^{a}
$$

Utilizando a relação acima para substituir $u_{f}^{a}$ por $\Delta u_{f}^{a}$, o termo definido na equação (5.6) pode ser escrito como:

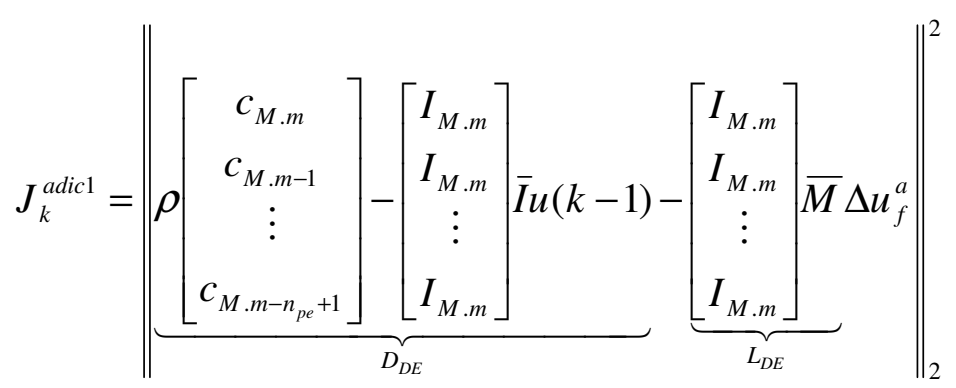

A equação (5.8) pode ser escrita na forma quadrática:

$$
J_{k}^{a d i c 1}=\Delta u_{f}^{a^{T}} L_{D E}{ }^{T} L_{D E} \Delta u_{f}^{a}-2 D_{D E}{ }^{T} L_{D E} \Delta u_{f}^{a}+D_{D E}{ }^{T} D_{D E}
$$


Utilizando a equação (5.9) e o modelo definido em (4.20), o problema de otimização do controlador SPC com excitação interna formado pela função objetivo (5.2) e pelas restrições (5.3) e (5.4) pode escrito como:

$$
\min _{\Delta u_{f}^{a}} \quad J_{k}^{e x c}=\Delta u_{f}^{a^{T}} H_{4} \Delta u_{f}^{a}+2 c_{f 4}{ }^{T} \Delta u_{f}^{a}+c_{4}
$$

sujeito a: $A_{\text {ineq } 4} \Delta u_{f}^{a} \leq b_{\text {ineq } 4}$

sendo:

$$
\begin{aligned}
& H_{4}=L_{u}^{a^{T}} \bar{Q} L_{u}^{a}+\bar{R}+P L_{D E}{ }^{T} L_{D E} ; \\
& c_{f 4}{ }^{T}=\left[Y_{k}+L_{w}^{a} \Delta w_{p}^{a}-Y_{s p}\right]^{T} \bar{Q} L_{u}^{a}-P D_{D E}{ }^{T} L_{D E} ; \\
& c_{4}=\left[Y_{k}+L_{w}^{a} \Delta w_{p}^{a}-Y_{s p}\right]^{T} \bar{Q}\left[Y_{k}+L_{w}^{a} \Delta w_{p}^{a}-Y_{s p}\right]+P D_{D E}{ }^{T} D_{D E} \text {; }
\end{aligned}
$$

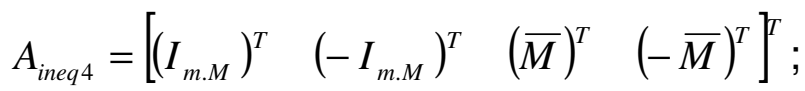

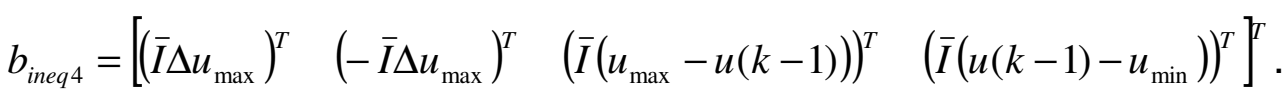

As matrizes $\bar{Q}, \bar{R}, Y_{s p}, \bar{M}$ e $\bar{I}$ são definidas pelas mesmas equações usadas no SPC original, apresentado na seção 4.2.1.

Uma grande vantagem do SPC apresentado acima é que, mesmo com a adição da excitação interna, o problema de otimização ainda pode ser resolvido através de algoritmos de programação quadrática. Esse controlador possui três parâmetros adicionais de sintonia: o número de direções menos excitadas $\left(n_{p e}\right)$, o nível de excitação $(\rho)$ e o peso do termo de excitação em relação ao termo referente ao controle $(P)$. É importante observar que o número total de direções calculadas pela decomposição SVD em (5.1) é $M \cdot m$, sendo esse o limite máximo na escolha de $n_{p e}$. Uma análise detalhada de cada um desses parâmetros será realizada posteriormente.

O controlador SPC com excitação interna calcula as direções menos excitadas a partir de uma decomposição SVD de uma matriz de dados passados. Portanto, 
primeiramente é necessário operar o SPC sem a condição de excitação, até que sejam coletados dados suficientes para realizar esse cálculo. Note ainda que os dados coletados não podem ser nulos. A decomposição SVD de dados nulos ou parcialmente nulos acarretaria em alguns valores singulares iguais à zero. Nesse caso, as respectivas direções desses valores singulares não teriam nenhuma relação lógica com a matriz de dados passados e, portanto, perderiam o significado de direções menos excitadas. Por esse motivo, em todas as simulações realizadas, que serão mostradas adiante, é adicionado um pequeno ruído na entrada do processo até o momento em que é iniciada a excitação pelo controlador SPC.

Durante o período em que a condição de excitação está inativa, não há necessidade de se manter os horizontes de predição $(n p)$ e de controle $(n c)$ iguais a $M$. Logo, nesse período é utilizado o controlador SPC desenvolvido na seção 4.2.2, o qual considera um sistema sem ação direta da entrada na saída e utiliza horizontes $n p$ e $n c$ independentes. Essa mudança é realizada, pois a flexibilização dos horizontes torna a resolução do problema de otimização mais rápida e melhora a estabilidade do controlador. Quando a condição de excitação é ativada, automaticamente os horizontes de predição e de controle passam a ser iguais a $M$, sendo que algumas matrizes devem então ser reconstruídas.

Com o intuito de ilustrar o comportamento do SPC com excitação interna, realizou-se uma simulação com o sistema da coluna debutanizadora da refinaria RPBC. Considerou-se o caso do controlador sem erro no modelo, sendo que tanto a planta como o modelo do controlador foram representados pelo Modelo C1. O modelo de predição em subespaços foi obtido pela identificação com os dados do experimento $A$ (Tabela 3.4). A sintonia usada no SPC está apresentada na Tabela 5.1.

$\mathrm{Na}$ simulação foi estudado o caso regulatório, com $y^{s p}=\left[\begin{array}{ll}0 & 0\end{array}\right]^{T}$. Até o instante 4000 minutos não houve excitação interna do controlador SPC e apenas um ruído aleatório de variância 0.0001 foi adicionado diretamente na entrada do processo. Em 4000 minutos, a condição de excitação foi ativada e permaneceu assim até o instante 10000 minutos. No cálculo das direções menos excitadas foram utilizadas matrizes de blocos de Hankel com $10 \cdot M$ colunas. 
Tabela 5.1 - Sintonia do controlador SPC com excitação interna.

\begin{tabular}{lcc}
\hline \multicolumn{1}{c}{ Parâmetro de Sintonia } & Símbolo & Valor \\
\hline Matriz de pesos das saídas & $Q$ & $\operatorname{diag}\left(\left[\begin{array}{ll}1 & 1\end{array}\right]\right)$ \\
\hline Matriz de pesos das entradas & $R$ & $\operatorname{diag}\left(\left[\begin{array}{ll}0.1 & 0.1\end{array}\right]\right)$ \\
\hline Limite máximo das entradas & $u_{\max }$ & {$\left[\begin{array}{ll}15 & 15\end{array}\right]^{T}$} \\
\hline Limite mínimo das entradas & $u_{\min }$ & {$\left[\begin{array}{ll}-10 & -10\end{array}\right]^{T}$} \\
\hline Variação máxima das entradas & $\Delta u_{\max }$ & {$\left[\begin{array}{ll}1 & 1\end{array}\right]^{T}$} \\
\hline Horizonte de predição & $n p$ & 20 \\
\hline Horizonte de controle & $n c$ & 7 \\
\hline Horizonte de dados passados & $M$ & 70 \\
\hline Número de direções menos excitadas & $n_{p e}$ & 300 \\
\hline Nível de excitação & $\rho$ & 0.1 \\
\hline Peso da excitação em relação ao controle & $P$ & 10 \\
\hline
\end{tabular}

A Figura 5.1 ilustra o comportamento das variáveis do sistema, enquanto a função objetivo do controlador é apresentada na Figura 5.2. Nessa última figura também são mostrados separadamente os termos da função objetivo referentes ao controle $\left(J_{k}\right)$ e à excitação $\left(P J_{k}^{\text {adic }}\right)$.
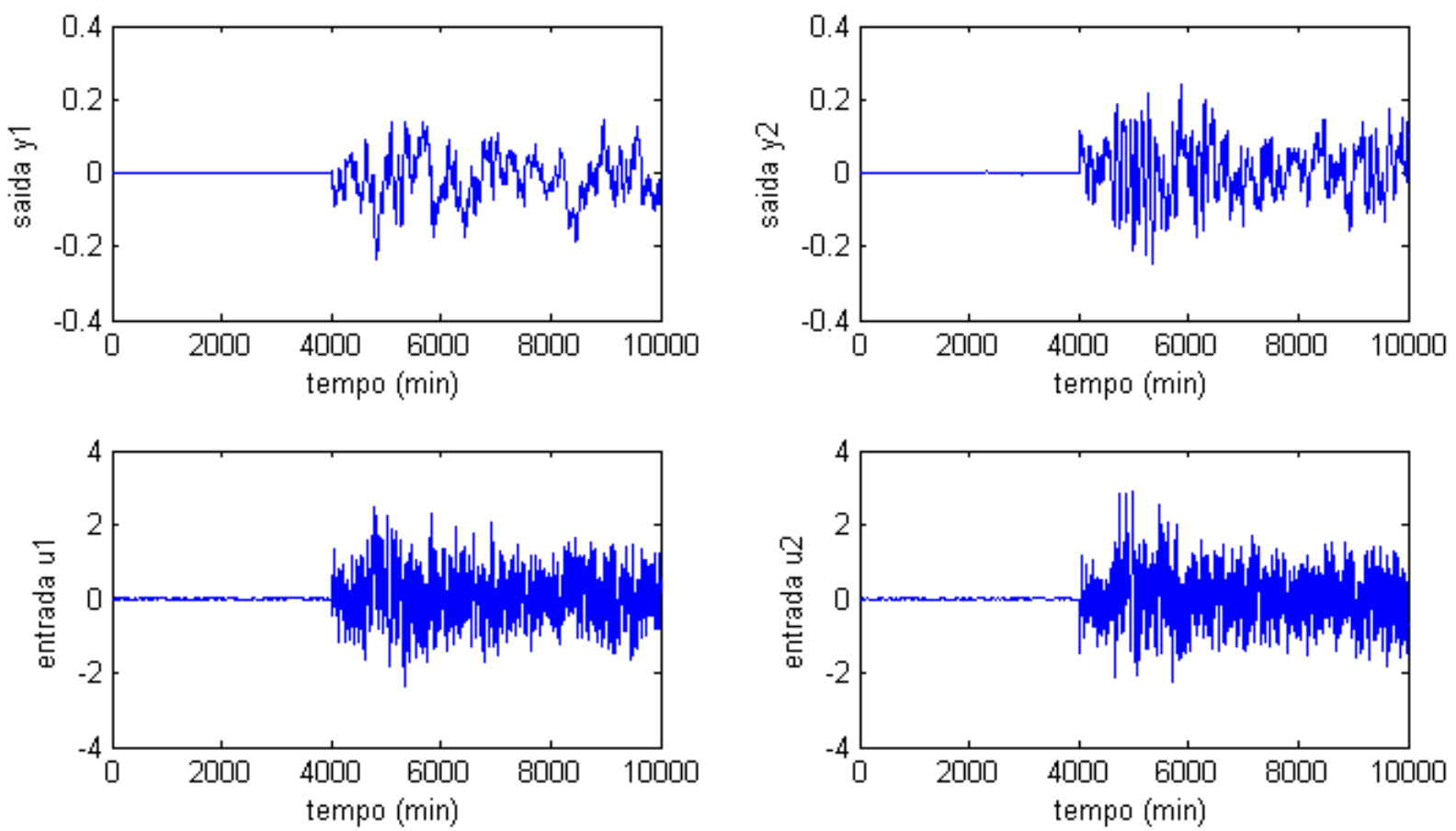

Figura 5.1 - Comportamento das variáveis do processo controlado pelo SPC com excitação direta na entrada. 


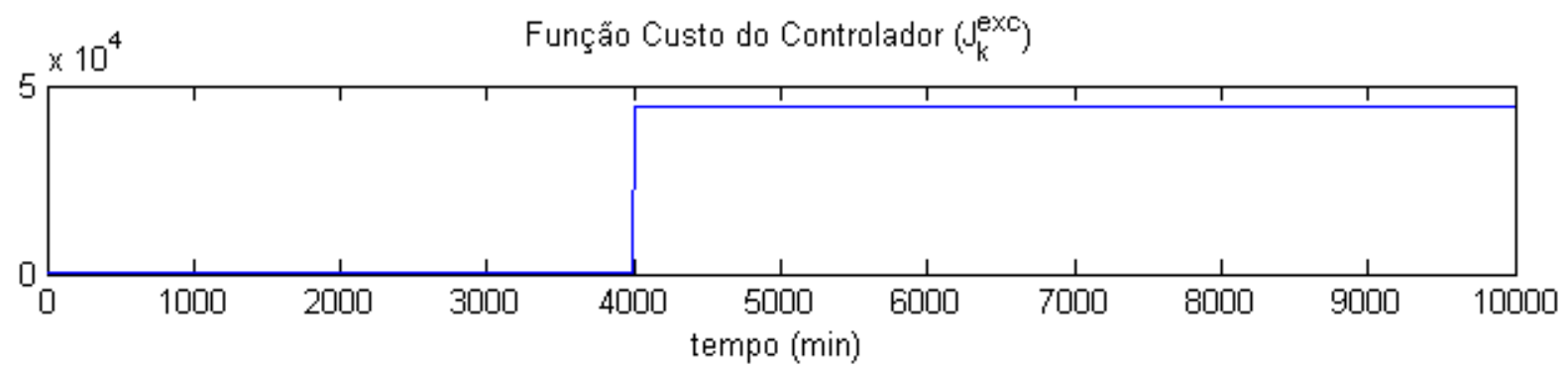

Parcela da Funçẫo Custo Referente ao Controle $\left(U_{k}\right)$
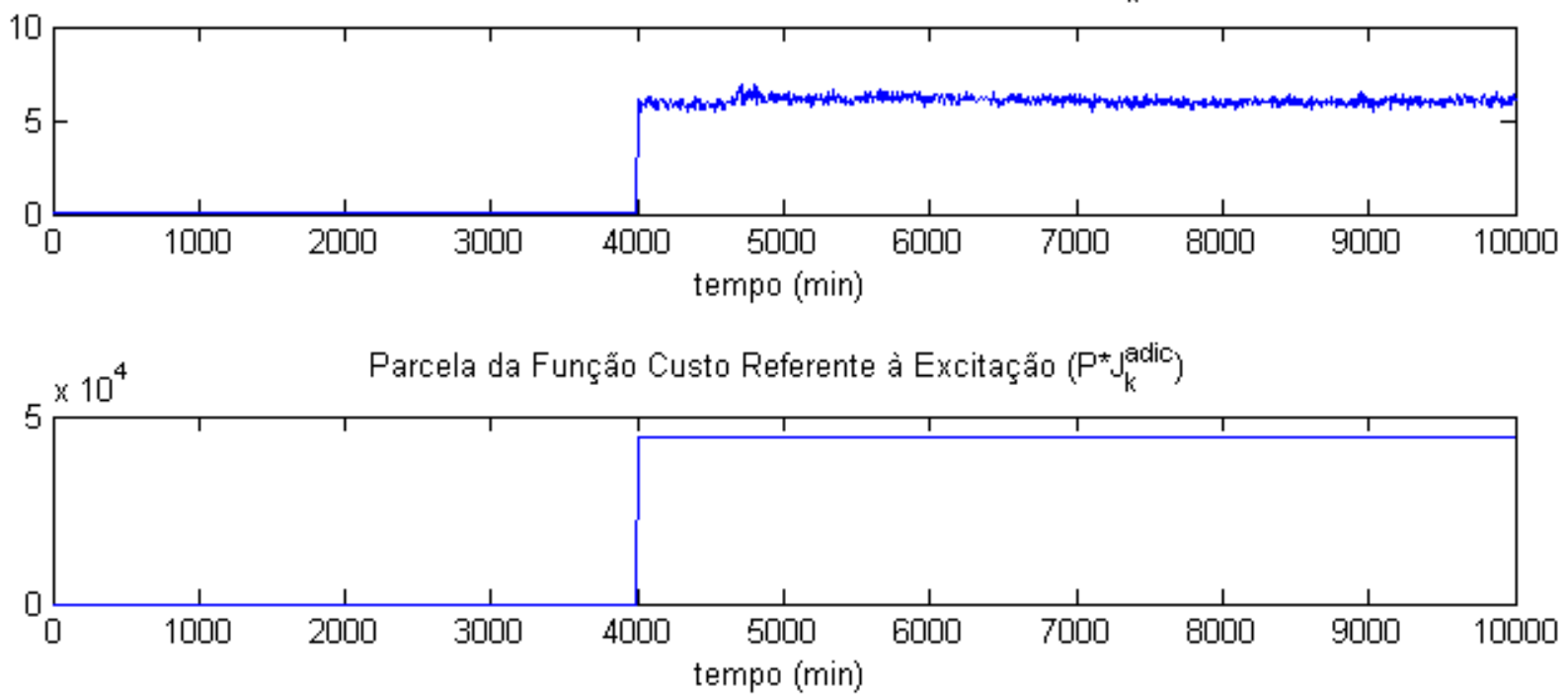

Figura 5.2 - Função objetivo do controlador SPC com excitação direta na entrada.

Analisando a Figura 5.2, nota-se que, no período de 0 a 4000 minutos, quando não há excitação por parte do controlador, a função custo é muito próxima de zero, sendo que a parcela referente à excitação é nula. A parcela referente ao controle não alcança o valor nulo pois há um pequeno erro entre as saídas e seus respectivos set-points. Esse erro é causado pelo ruído aleatório adicionado na entrada do processo. A partir do instante 4000 minutos, inicia-se a excitação interna do controlador. Verifica-se que as variações nas entradas e nas saídas passam a ter amplitudes maiores, porém as oscilações das variáveis controladas permanecem em torno de seus valores de referência (Figura 5.1).

A Figura 5.2 mostra que após o instante 4000 minutos a função objetivo aumenta drasticamente. A parcela referente ao controle se distancia um pouco de zero, pois a excitação do sistema gera um erro entre as saídas e seus set-points. Porém, a parcela referente à excitação é extremamente mais alta, sendo responsável pelo valor elevado da função objetivo. Esse comportamento deve-se principalmente ao termo constante dessa parcela $\left(P D_{D E}{ }^{T} D_{D E}\right)$. 
A partir da definição de $D_{D E}$ (5.8), percebe-se que o termo $P D_{D E}{ }^{T} D_{D E}$ quantifica a diferença entre a última entrada implementada e as direções menos excitadas. Como a última entrada é única, mesmo que ela seja idêntica a uma direção menos excitada, as diferenças em relação a todas as outras direções serão diferentes de zero. Além disso, cada direção é formada por um vetor com $M$ blocos de linhas, totalizando $M \cdot m$ linhas, e a diferença apresentada no termo $D_{D E}$ é contabilizada para cada componente de cada direção. Assim, o termo constante da parcela referente à excitação é proporcional ao horizonte do modelo de predição $(M)$, ao número de direções consideradas $\left(n_{p e}\right)$ e ao número de entradas $(m)$.

Por fim, é possível observar na Figura 5.1 que, durante o período de excitação interna do SPC, as entradas do processo são alteradas a cada período de amostragem, causando oscilações de alta freqüência nas variáveis controladas. Como a dinâmica do processo é lenta, esse tipo de excitação não é ideal, pois permite apenas que as respostas rápidas do sistema sejam observadas.

Para corrigir o problema descrito acima, é proposto neste trabalho que as direções menos excitadas sejam calculadas em intervalos de tempo maiores e entre cada cálculo seus valores sejam mantidos constantes. O intervalo de tempo de cálculo das direções $\left(T_{c d}\right)$ é um novo parâmetro de sintonia e deve ser ajustado conforme a dinâmica do processo. Caso seja escolhido $T_{c d}=1$ período de amostragem, 0 controlador SPC com cálculo das direções a cada instante é obtido. Para ilustrar o efeito desse parâmetro, foi repetida a simulação da coluna debutanizadora para o caso de $T_{c d}=200$ minutos. O resultado obtido, apenas no intervalo em que a excitação interna do SPC esteve ativa, é mostrado na Figura 5.3.

Como as direções menos excitadas são calculadas apenas a cada 40 períodos de amostragem (200 min), observa-se, na Figura 5.3, que as entradas se comportam com alterações em degraus de comprimentos iguais a 200 minutos. Nota-se ainda que a sintonia escolhida não gera direções menos excitadas que ultrapassam os limites das variáveis manipuladas e/ou os limites de máxima variação dessas variáveis. Se a máxima variação de uma entrada fosse um fator limitante, seria 
observado nessa variável um comportamento em rampa até o alcance da direção desejada, sendo que, a partir desse momento, seu valor permaneceria constante enquanto novas direções não fossem calculadas.

Os degraus observados na Figura 5.3 para as variáveis manipuladas não são observados nas saídas do processo, principalmente para a saída $y_{1}$. Como a dinâmica do sistema é lenta, apesar das entradas permanecerem constantes, as saídas "caminham" vagarosamente para seus novos valores. Isso permite que todo o comportamento do processo seja registrado. Além disso, a adição do parâmetro $T_{c d}$ no SPC com excitação interna diminuiu significativamente o seu tempo de execução, pois a decomposição SVD realizada no cálculo das direções menos excitadas requer grande esforço computacional.
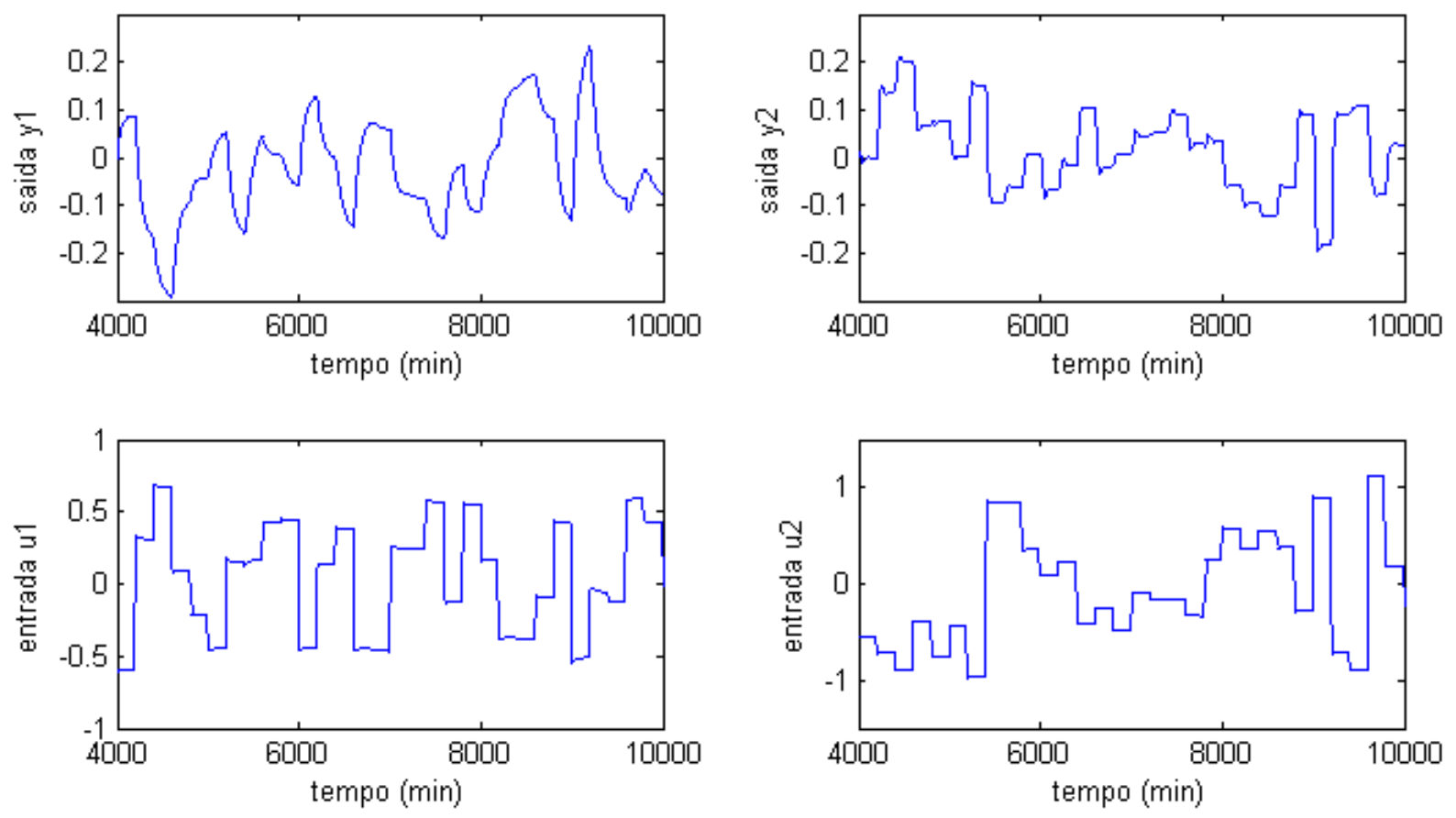

Figura 5.3 - Controlador SPC com excitação direta na entrada e cálculo das direções menos excitadas a cada 200 minutos.

\subsubsection{Cálculo do estado estacionário}

Na seção anterior, o controlador SPC com excitação interna foi testado apenas para o caso regulatório. Para que ele seja usado no caso de set-points diferentes da 
origem, é necessário calcular o estado estacionário. Isso porque as direções menos excitadas são impostas diretamente para a entrada do processo, que consiste em um valor relativo ao valor absoluto da entrada no estado estacionário. Muitos artigos assumem que esse cálculo é realizado, porém não foi encontrado na literatura nenhum cálculo desse tipo aplicado diretamente para o modelo de predição em subespaços. Por esse motivo, foi desenvolvido neste trabalho um método de obtenção do estado estacionário.

O objetivo do método proposto é encontrar o valor absoluto da entrada no regime permanente $\left(u_{s s}\right)$ correspondente ao set-point da saída $\left(y^{s p}\right)$, a partir das matrizes do modelo de predição em subespaços. Utiliza-se nesse desenvolvimento o modelo de predição na forma em que ele é identificado (equação (4.1)):

$$
\hat{y}_{f}=L_{w} w_{p}+L_{u} u_{f}
$$

Assumindo que a planta esteja em regime permanente, todas as entradas e saídas passadas e futuras permanecem constantes e a equação do modelo (4.1) fica:

$$
\left[\begin{array}{c}
y^{S P} \\
y^{S P} \\
\vdots \\
y^{S P}
\end{array}\right]=L_{w}\left[\begin{array}{c}
y^{S P} \\
\vdots \\
y^{S P} \\
u_{s s} \\
\vdots \\
u_{s s}
\end{array}\right]+L_{u}\left[\begin{array}{c}
u_{s s} \\
u_{s s} \\
\vdots \\
u_{s s}
\end{array}\right]
$$

Pode-se separar a matriz $L_{w}$ em duas parcelas: a primeira referente às saídas passadas e a segunda referente às entradas passadas:

$$
L_{w}=\left[\begin{array}{ll}
L_{w 1} & L_{w 2}
\end{array}\right]
$$

sendo $L_{w 1} \in R^{M \cdot l \times M \cdot l}$ e $L_{w 2} \in R^{M \cdot l \times M \cdot m}$.

Substituindo a equação (5.11) na equação (5.10), tem-se: 


$$
\left[\begin{array}{c}
y^{S P} \\
y^{S P} \\
\vdots \\
y^{S P}
\end{array}\right]=L_{w 1}\left[\begin{array}{c}
y^{S P} \\
y^{S P} \\
\vdots \\
y^{S P}
\end{array}\right]+L_{w 2}\left[\begin{array}{c}
u_{s s} \\
u_{s s} \\
\vdots \\
u_{s s}
\end{array}\right]+L_{u}\left[\begin{array}{c}
u_{s s} \\
u_{s s} \\
\vdots \\
u_{s s}
\end{array}\right]
$$

Rearranjando a equação acima se obtém:

$$
\left[\begin{array}{c}
u_{s s} \\
u_{s s} \\
\vdots \\
u_{s s}
\end{array}\right]=\left(L_{u}+L_{w 2}\right)^{-1}\left(I_{l . M}-L_{w 1}\right)\left[\begin{array}{c}
y^{S P} \\
y^{S P} \\
\vdots \\
y^{S P}
\end{array}\right]
$$

O valor da entrada no estado estacionário $\left(u_{s s}\right)$ pode ser determinado pelo primeiro bloco de linhas da equação acima. Esse cálculo deve ser refeito toda vez que houver alteração no set-points das variáveis controladas ou quando o modelo for reidentificado.

O procedimento acima foi testado para o sistema da coluna debutanizadora e apresentou um resultado satisfatório. É importante lembrar que, como todo cálculo de estado estacionário, deve-se adicionar o erro de predição no cálculo, para que o resultado obtido não apresente bias devido à imprecisão do modelo.

\subsection{CONTROLADOR COM EXCITAÇÃO NO INCREMENTO DA ENTRADA}

O controlador SPC apresentado na seção anterior necessita do cálculo do estado estacionário, que pode ser um procedimento complicado e impreciso. Se não houver precisão suficiente nesse cálculo, a excitação do controlador pode apresentar offset, isto é, as oscilações das saídas podem não ocorrer em torno de seus set-points. Para contornar esse problema, foi desenvolvido um novo método no qual não é necessário utilizar o estado estacionário. 
O SPC com excitação interna apresentado a seguir consiste em impor as direções menos excitadas para as variações das ações de controle futuras. Desse modo, a referência utilizada no termo de excitação deixa de ser a entrada no estado estacionário e passa a ser a entrada no instante anterior. O problema de otimização a ser resolvido em cada instante é:

$$
\begin{aligned}
\min _{\Delta u_{f}^{a}} & J_{k}^{e x c}=J_{k}+P J_{k}^{a d i c 2} \\
& \text { sujeito a: } \\
& -\Delta u_{\max } \leq \Delta u(k+j \mid k) \leq \Delta u_{\max }, \quad j=0, \ldots, M-1 \\
& u_{\min } \leq u(k+j \mid k) \leq u_{\max }, \quad j=0, \ldots, M-1
\end{aligned}
$$

sendo $J_{k}$ a função custo do controlador SPC sem excitação apresentada na equação (5.5). O termo adicional $J_{k}^{\text {adic2 }}$ pode ser escrito como:

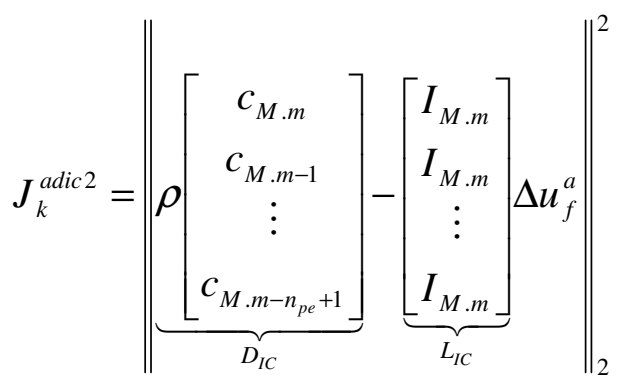

A equação (5.16) pode ser escrita na forma quadrática:

$$
J_{k}^{a d i c 2}=\Delta u_{f}^{a T} L_{I C}{ }^{T} L_{I C} \Delta u_{f}^{a}-2 D_{I C}{ }^{T} L_{I C} \Delta u_{f}^{a}+D_{I C}{ }^{T} D_{I C}
$$

Utilizando a equação acima e o modelo definido em (4.20), o problema de otimização do controlador SPC com excitação interna formado pela função objetivo (5.13) e pelas restrições (5.14) e (5.15) pode escrito como:

$$
\min _{\Delta u_{f}^{a}} J_{k}^{e x c}=\Delta u_{f}^{a^{T}} H_{5} \Delta u_{f}^{a}+2 c_{f 5}{ }^{T} \Delta u_{f}^{a}+c_{5}
$$

sujeito a: $A_{\text {ineq } 5} \Delta u_{f}^{a} \leq b_{\text {ineq } 5}$ 
sendo:

$$
\begin{aligned}
& H_{5}=L_{u}^{a^{T}} \bar{Q} L_{u}^{a}+\bar{R}+P L_{I C}{ }^{T} L_{I C} ; \\
& c_{f 5}{ }^{T}=\left[Y_{k}+L_{w}^{a} \Delta w_{p}^{a}-Y_{s p}\right]^{T} \bar{Q} L_{u}^{a}-P D_{I C}{ }^{T} L_{I C} ; \\
& c_{5}=\left[Y_{k}+L_{w}^{a} \Delta w_{p}^{a}-Y_{s p}\right]^{T} \bar{Q}\left[Y_{k}+L_{w}^{a} \Delta w_{p}^{a}-Y_{s p}\right]+P D_{I C}{ }^{T} D_{I C} ;
\end{aligned}
$$

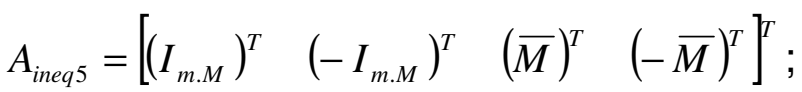

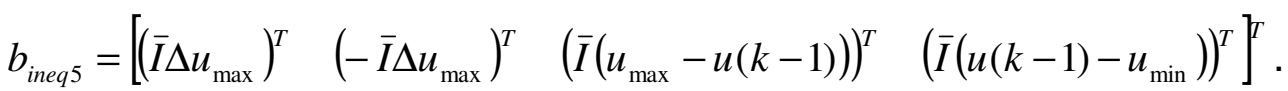

As matrizes $\bar{Q}, \bar{R}, Y_{s p}, \bar{M}$ e $\bar{I}$ são definidas pelas mesmas equações usadas no SPC original, apresentado na seção 4.2.1.

Note que o problema proposto acima possui o mesmo formato do problema do controlador SPC com excitação direta na entrada. A única diferença está no cálculo dos coeficientes referentes ao termo de excitação $\left(D_{I C}\right.$ e $\left.L_{I C}\right)$. Como no SPC com excitação no incremento da entrada o ponto de referência na excitação corresponde ao estado do sistema no instante anterior, é importante que as variáveis controladas estejam em seus set-points no momento em que a condição de excitação é ativada.

Realizou-se uma simulação com o sistema da coluna debutanizadora para ilustrar o comportamento do SPC com excitação no incremento da entrada. Foram consideradas as mesmas condições das simulações realizadas na seção 5.1.2. A sintonia utilizada também foi a mesma (Tabela 5.1) e optou-se por usar $T_{c d}=200$ minutos. A Figura 5.4 mostra o resultado obtido no período em que a excitação interna do controlador foi mantida ativa.

Analisando a Figura 5.4, é possível observar que as oscilações das saídas ocorrem em torno de seus set-points. É importante ressaltar que não há nenhuma restrição no controlador SPC que garanta esse comportamento. Entretanto, essa tendência é observada na prática, mostrando que as direções menos excitadas calculadas sucessivamente tendem a ter média zero. Percebe-se que as oscilações geradas pelo SPC com excitação no incremento da entrada são menos regulares e possuem amplitudes maiores do que as oscilações geradas pelo controlador SPC proposto na 
seção 5.1. Isso pode ser comprovado comparando-se a Figura 5.4 e a Figura 5.3. Porém, esse comportamento não representa um problema, pois o nível de excitação pode ser refinado pelos parâmetros de sintonia.

Ainda na Figura 5.4, nota-se que, no novo método de excitação, as entradas perderam a característica de alterações em degraus. O ponto de referência no termo de excitação é a última entrada implementada e, portanto, tem seu valor alterado a cada instante de amostragem. Dessa forma, nunca se alcança a direção desejada, sendo que as variáveis manipuladas permanecem seguindo um mesmo sentido até o momento em que novas direções menos excitadas são calculadas ou até elas atingirem seus limites. Para contornar esse problema será desenvolvido na seção seguinte outro método SPC com excitação interna.
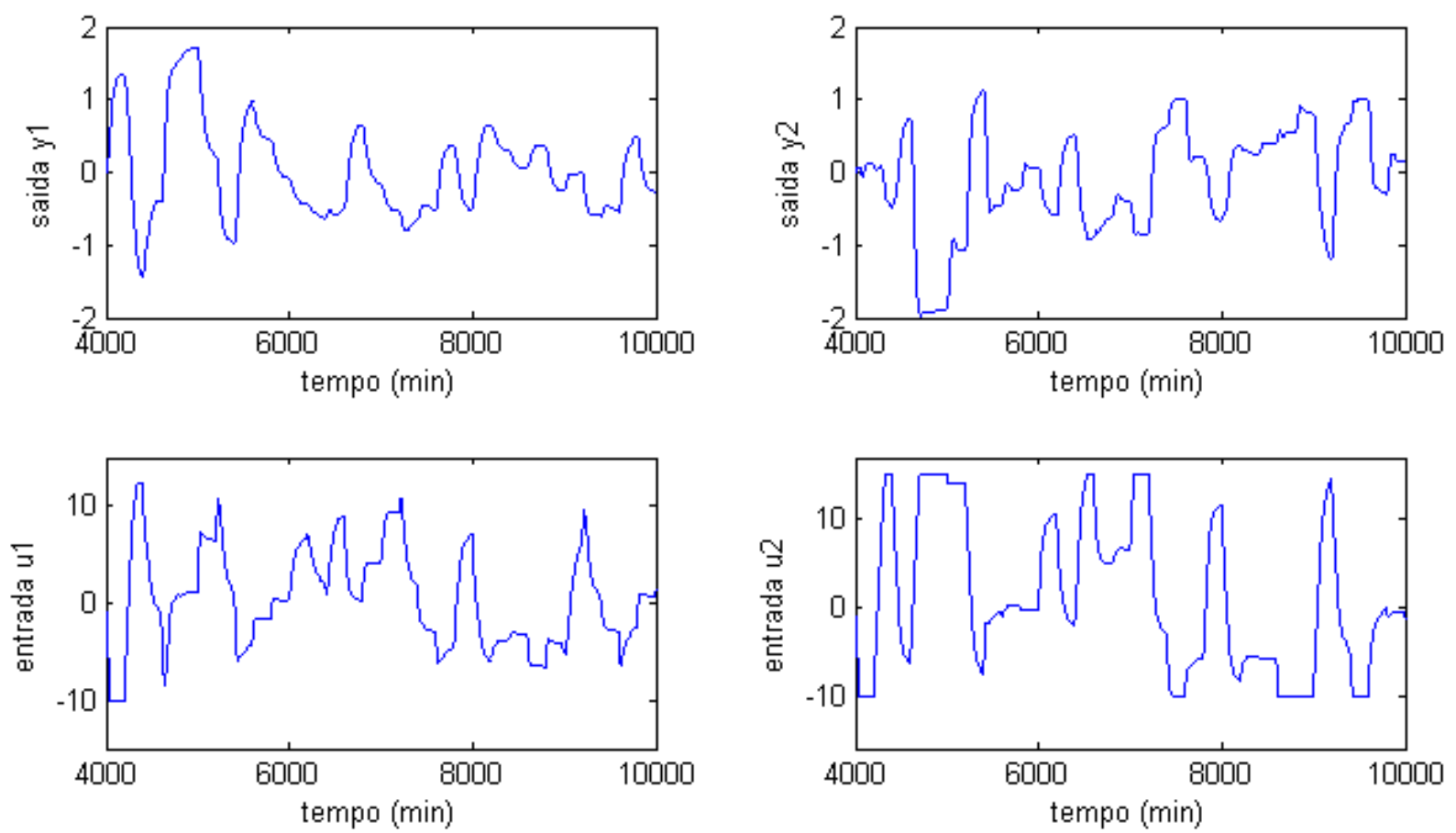

Figura 5.4 - Controlador SPC com excitação no incremento da entrada e cálculo das direções menos excitadas a cada 200 minutos.

\subsection{CONTROLADOR COM CÁLCULO DO VALOR DE REFERÊNCIA}

O controlador SPC com excitação direta na entrada, proposto na seção 5.1 , tem um inconveniente, pois necessita do cálculo do estado estacionário do sistema. Para 
solucionar esse problema, foi desenvolvido na seção 5.2 um novo método de excitação. Porém, esse novo algoritmo não gera variações em degraus nas entradas do processo. A seguir será apresentado um terceiro método SPC com excitação interna que reúne as vantagens de ambos os métodos anteriores, eliminado os inconvenientes descritos acima.

O novo algoritmo de excitação interna propõe que as direções menos excitadas sejam impostas às entradas futuras do processo relativas a um valor de referência. Esse valor deve ser a última ação de controle implementada no instante em que as direções foram calculadas. Dessa forma, as direções menos excitadas e o ponto de referência do termo de excitação são atualizados na mesma freqüência (definida pelo parâmetro $T_{c d}$ ). O problema de otimização a ser resolvido em cada instante é:

$$
\begin{aligned}
\min _{\Delta u_{f}^{a}} & J_{k}^{e x c}=J_{k}+P J_{k}^{\text {adic3 }} \\
& \text { sujeito a: } \\
& -\Delta u_{\max } \leq \Delta u(k+j \mid k) \leq \Delta u_{\max }, \quad j=0, \ldots, M-1 \\
& u_{\min } \leq u(k+j \mid k) \leq u_{\max }, \quad j=0, \ldots, M-1
\end{aligned}
$$

sendo $J_{k}$ a função custo do controlador SPC sem excitação apresentada na equação (5.5). O termo adicional $J_{k}^{\text {adic3 }}$ pode ser escrito como:

$$
J_{k}^{\text {adic3 }}=\left\|\rho\left[\begin{array}{c}
c_{M . m} \\
c_{M . m-1} \\
\vdots \\
c_{M \cdot m-n_{p e}+1}
\end{array}\right]-\left[\begin{array}{c}
I_{M . m} \\
I_{M . m} \\
\vdots \\
I_{M \cdot m}
\end{array}\right]\left(u_{f}^{a}-\bar{I} u_{r e f}\right)\right\|^{2}
$$

Utilizando a equação (5.7) para substituir $u_{f}^{a}$ por $\Delta u_{f}^{a}$, o termo definido na equação (5.21) pode ser escrito como: 


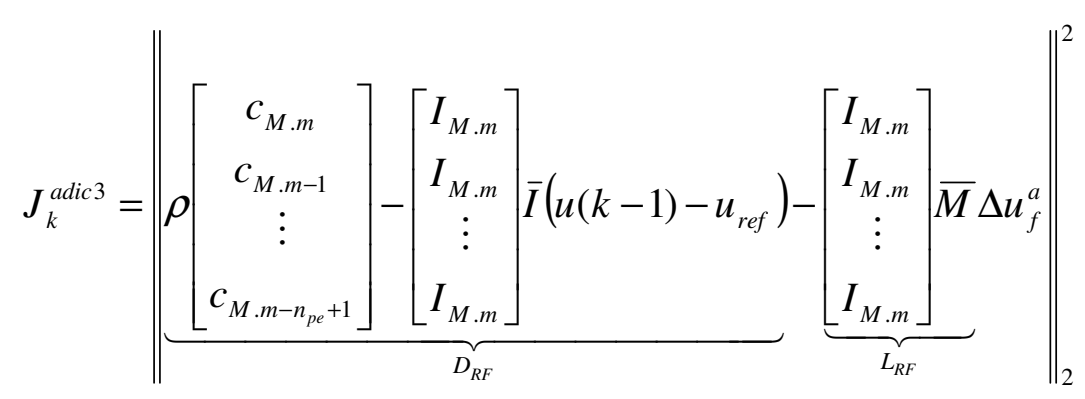

A equação (5.22) pode ser escrita na forma quadrática:

$$
J_{k}^{a d i c 3}=\Delta u_{f}^{a^{T}} L_{R F}{ }^{T} L_{R F} \Delta u_{f}^{a}-2 D_{R F}{ }^{T} L_{R F} \Delta u_{f}^{a}+D_{R F}{ }^{T} D_{R F}
$$

Utilizando a equação acima e o modelo definido em (4.20), o problema de otimização do controlador SPC com excitação interna formado pela função objetivo (5.18) e pelas restrições (5.19) e (5.20) pode escrito como:

$$
\min _{\Delta u_{f}^{a}} \quad J_{k}^{e x c}=\Delta u_{f}^{a^{T}} H_{6} \Delta u_{f}^{a}+2 c_{f 6}{ }^{T} \Delta u_{f}^{a}+c_{6}
$$

sujeito a: $A_{\text {ineq } 6} \Delta u_{f}^{a} \leq b_{\text {ineq } 6}$

sendo:

$$
\begin{aligned}
& H_{6}=L_{u}^{a} \bar{Q} L_{u}^{a}+\bar{R}+P L_{R F}{ }^{T} L_{R F} ; \\
& c_{f 6}{ }^{T}=\left[Y_{k}+L_{w}^{a} \Delta w_{p}^{a}-Y_{s p}\right]^{T} \bar{Q} L_{u}^{a}-P D_{R F}{ }^{T} L_{R F} ; \\
& c_{6}=\left[Y_{k}+L_{w}^{a} \Delta w_{p}^{a}-Y_{s p}\right]^{T} \bar{Q}\left[Y_{k}+L_{w}^{a} \Delta w_{p}^{a}-Y_{s p}\right]+P D_{R F}{ }^{T} D_{R F} ;
\end{aligned}
$$

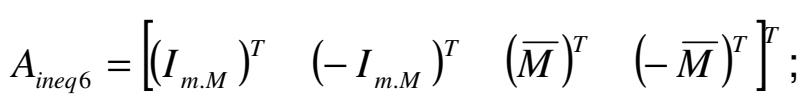

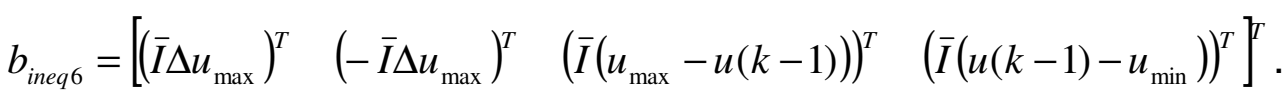

As matrizes $\bar{Q}, \bar{R}, Y_{s p}, \bar{M}$ e $\bar{I}$ são definidas pelas mesmas equações usadas no SPC original, apresentado na seção 4.2.1.

O problema proposto acima mantém o mesmo formato dos métodos anteriores. Analogamente ao controlador SPC com excitação no incremento da entrada, esse 
método não relaciona a excitação com o estado correspondente aos set-points das variáveis controladas, e por isso, é importante que essas variáveis estejam em seus valores desejados no momento em que a condição de excitação é ativada.

Realizou-se uma simulação com o sistema da coluna debutanizadora para ilustrar o comportamento do SPC com cálculo do valor de referência. Foram consideradas as mesmas condições das simulações realizadas na seção 5.1.2. A sintonia utilizada também foi a mesma (Tabela 5.1) e optou-se por usar $T_{c d}=200$ minutos. A Figura 5.5 mostra o resultado obtido no período em que a excitação interna do controlador foi mantida ativa.
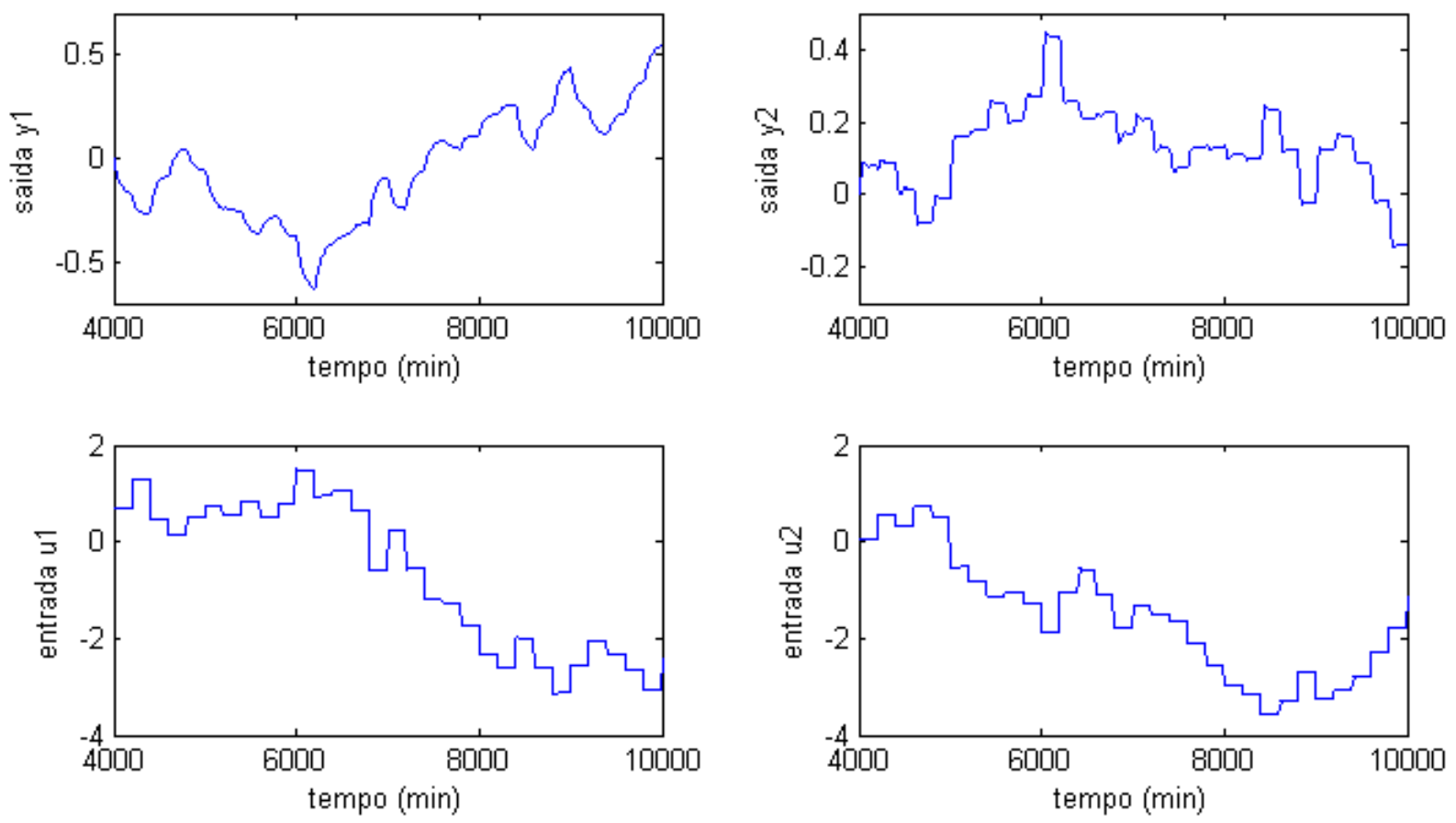

Figura 5.5 - Controlador SPC com cálculo do valor de referência e cálculo das direções menos excitadas a cada 200 minutos.

Nota-se, pela Figura 5.5, que as oscilações geradas são menos regulares que aquelas observadas no controlador SPC com excitação direta na entrada. Contudo, os sinais das saídas tendem a se manter em torno de seus set-points, apesar de não haver garantia desse comportamento. Além disso, as alterações em degraus nas manipuladas podem novamente ser observadas. 


\subsection{SIMULAÇÕES E ANÁLISES}

O SPC com excitação interna foi testado para o sistema da coluna debutanizadora, apresentado na seção 3.3.1. Primeiramente diversas simulações foram realizadas no intuito de analisar detalhadamente a influência de cada parâmetro de sintonia. Posteriormente, aplicou-se o controlador com reidentificação do modelo em malha fechada, verificando como esse procedimento afeta o desempenho do SPC.

\subsubsection{Análise dos parâmetros de sintonia}

Os três algoritmos de excitação interna para o método SPC propostos neste capítulo possuem quatro parâmetros de sintonia adicionais: o número de direções menos excitadas $\left(n_{p e}\right)$, o nível de excitação $(\rho)$, o peso do termo de excitação em relação ao termo referente ao controle $(P)$ e o intervalo de tempo de cálculo das direções $\left(T_{c d}\right)$. A seguir cada um desses parâmetros será analisado detalhadamente.

Diversas simulações com o sistema da coluna debutanizadora foram realizadas nas quais uma sintonia base foi mantida, variando-se apenas o parâmetro de sintonia estudado. Considerou-se o caso nominal, sendo que o modelo do controlador e a planta foram representados pelo Modelo C1. O modelo de predição em subespaços foi obtido pela identificação com os dados do experimento $A$ (Tabela 3.4). Optou-se por utilizar o controlador SPC com excitação direta na entrada, pois nesse algoritmo a excitação possui um ponto de referência fixo (o estado estacionário), gerando oscilações mais regulares. Essa característica permite uma melhor análise da influência individual de cada parâmetro.

Em cada teste estudou-se o caso de $y^{s p}=\left[\begin{array}{ll}0 & 0\end{array}\right]^{T}$, com a condição de excitação sendo ativada a partir do instante 4000 minutos. Até esse instante foi adicionado diretamente na entrada do processo um ruído aleatório de variância 0.0001. A sintonia base utilizada está mostrada na Tabela 5.2. 
Tabela 5.2 - Sintonia base do controlador SPC para análise dos parâmetros de sintonia.

\begin{tabular}{lcc}
\hline \multicolumn{1}{c}{ Parâmetro de Sintonia } & Símbolo & Valor \\
\hline Matriz de pesos das saídas & $Q$ & $\operatorname{diag}\left(\left[\begin{array}{ll}1 & 1\end{array}\right]\right)$ \\
\hline Matriz de pesos das entradas & $R$ & $\operatorname{diag}\left(\left[\begin{array}{ll}0.1 & 0.1\end{array}\right]\right)$ \\
\hline Limite máximo das entradas & $u_{\max }$ & {$\left[\begin{array}{ll}15 & 15\end{array}\right]^{T}$} \\
\hline Limite mínimo das entradas & $u_{\min }$ & {$\left[\begin{array}{ll}10 & -10\end{array}\right]^{T}$} \\
\hline Variação máxima das entradas & $\Delta u_{\max }$ & {$\left[\begin{array}{ll}1 & 1\end{array}\right]^{T}$} \\
\hline Horizonte de predição & $n p$ & 20 \\
\hline Horizonte de controle & $n c$ & 7 \\
\hline Horizonte de dados passados & $M$ & 70 \\
\hline Número de direções menos excitadas & $n_{p e}$ & 10 \\
\hline Nível de excitação & $\rho$ & 1 \\
\hline Peso da excitação em relação ao controle & $P$ & 5 min
\end{tabular}

Em cada análise serão apresentados os comportamentos das variáveis do processo e da função objetivo do controlador somente no intervalo de tempo em que a condição de excitação permaneceu ativa. Os resultados estão mostrados a seguir.

Estudo 1: Análise do número de direções menos excitadas $\left(n_{p e}\right)$.
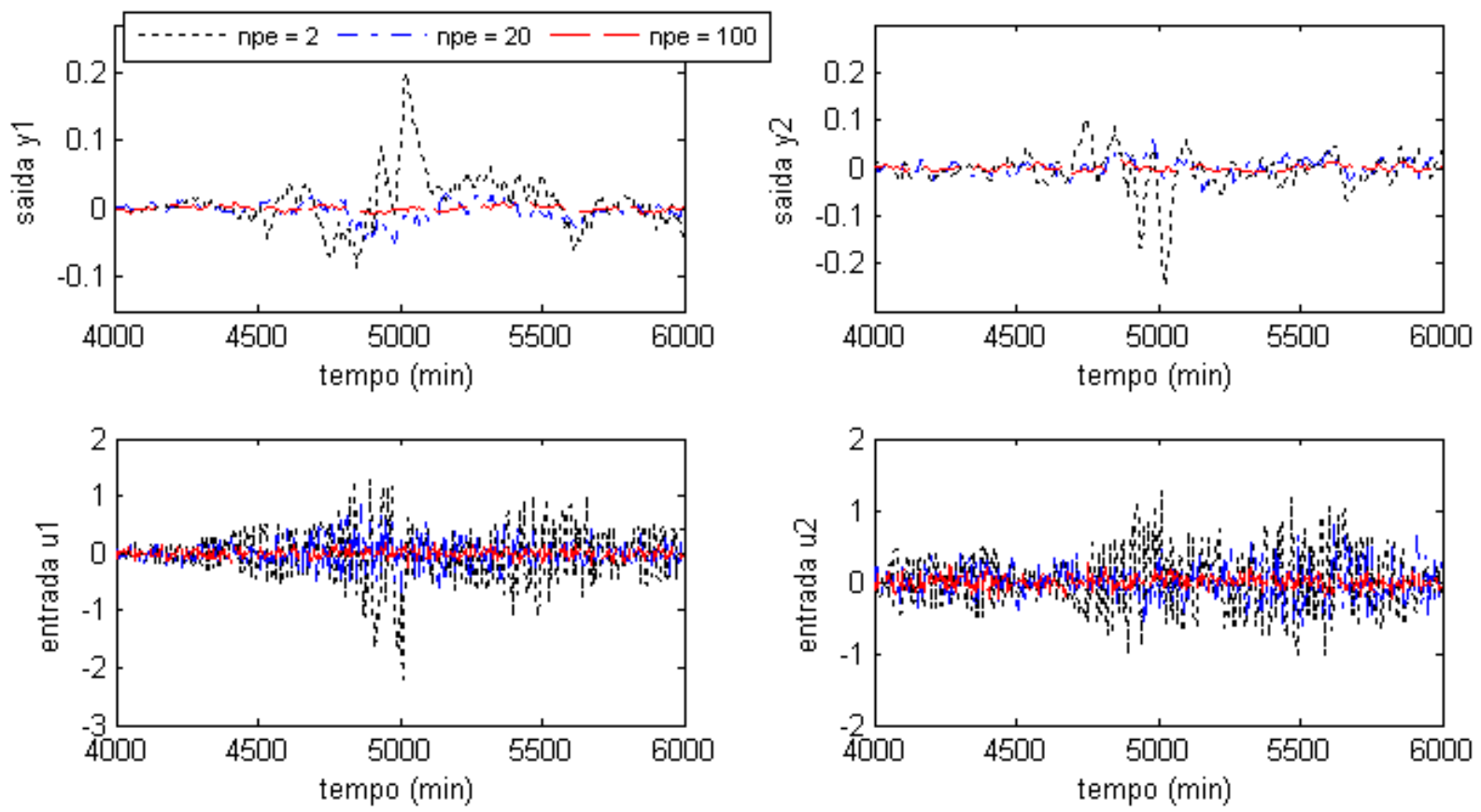

Figura 5.6 - Controlador SPC com excitação interna. Análise do número de direções menos excitadas. 
Funçẫo Custo do Controlador $\left(J_{\mathrm{k}}^{\text {exc }}\right)$

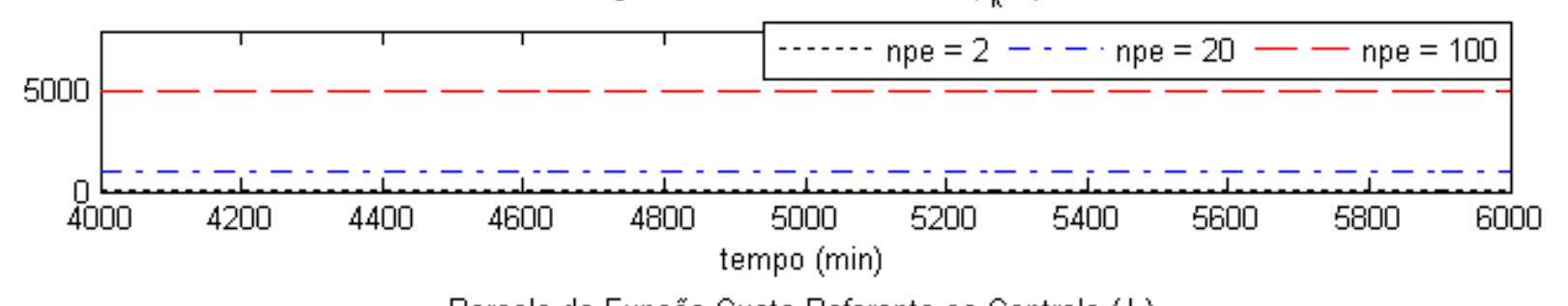

Parcela da Funçẫo Custo Referente ao Controle $\left(J_{k}\right)$

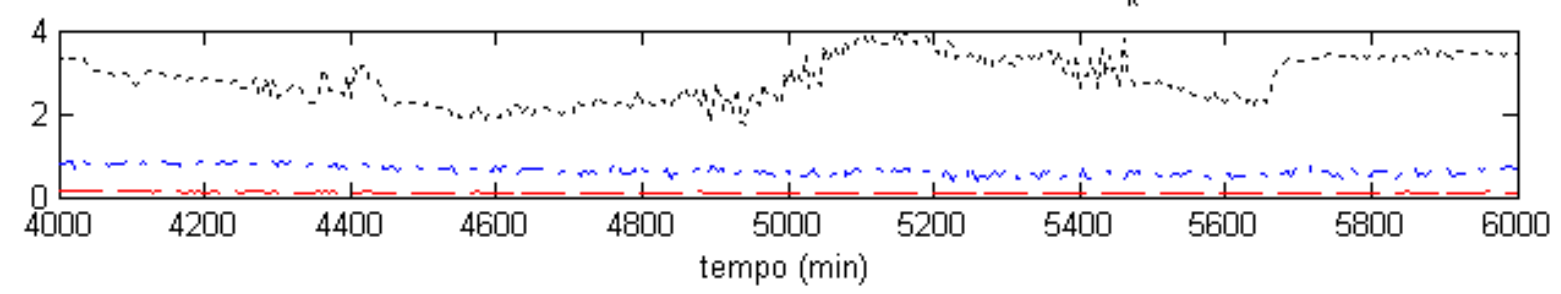

Parcela da Funçẫo Custo Referente à Excitaçẫo ( $\mathrm{P}^{*} \mathrm{~J}_{\mathrm{k}}^{\text {adic }}$ )

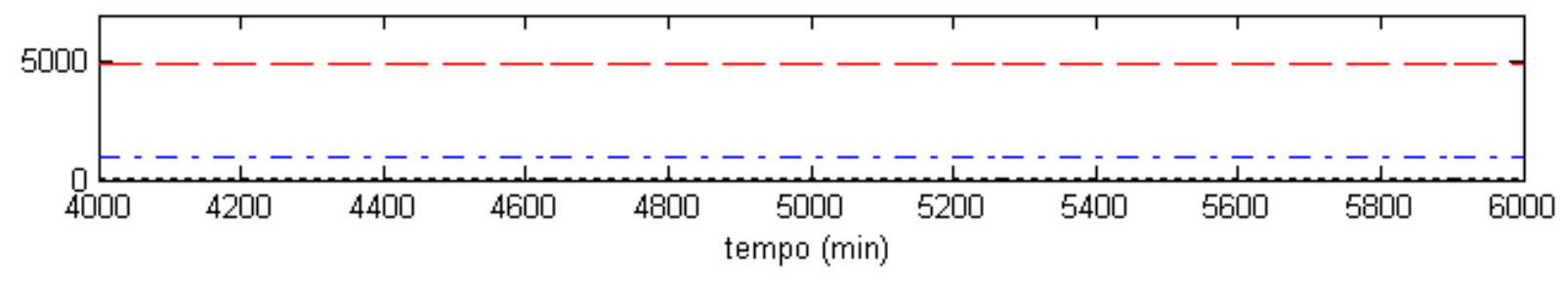

Figura 5.7 - Função objetivo do controlador SPC com excitação interna. Análise do número de direções menos excitadas.

As simulações mostraram que o aumento do número de direções menos excitadas diminui a amplitude das oscilações nas saídas do processo e, portanto, desfavorece a excitação. Esse fato pode ser observado na Figura 5.7: nota-se que a função custo referente ao controle $\left(J_{k}\right)$ diminui conforme o parâmetro $n_{p e}$ aumenta. No caso de $n_{p e}=100$, essa parcela fica muito próxima de zero, mostrando que as variáveis controladas não se distanciam muito de seus set-points e logo, não são suficientemente excitadas.

O resultado descrito acima era esperado, pois quando são consideradas muitas direções menos excitadas, há uma concorrência na determinação da direção que o vetor de entradas futuras deve seguir. No caso de uma única direção menos excitada, o termo da função custo referente à excitação força o vetor de entradas futuras (de dimensão $M \cdot m$ ) nessa direção (também de dimensão $M \cdot m$ ). Para qualquer outro valor de $n_{p e}$, as entradas do processo não conseguem seguir uma única direção. Essa concorrência faz com que as entradas futuras assumam um valor médio das direções consideradas, diminuindo o nível de excitação. Além disso, 
quando $n_{p e}$ é excessivamente elevado, direções que já possuem um grau considerável de excitação são adicionadas no problema de otimização, o que gera uma inércia sobre as ações de controle.

Ainda na Figura 5.7, nota-se que a parcela da função objetivo referente à excitação aumenta rapidamente com o aumento do parâmetro $n_{p e}$. Esse comportamento devese principalmente ao aumento sofrido pelo termo constante dessa parcela $\left(P D_{D E}{ }^{T} D_{D E}\right)$. Conforme já discutido na seção 5.1 .2 , o termo $P D_{D E}{ }^{T} D_{D E}$ é proporcional ao horizonte do modelo de predição $(M)$, ao número de direções consideradas $\left(n_{p e}\right)$ e ao número de entradas $(m)$.

Durante a execução das simulações, observou-se uma ligação entre o número de direções menos excitadas $\left(n_{p e}\right)$ e o intervalo de cálculo das direções $\left(T_{c d}\right)$. Quando se considera um valor elevado para $T_{c d}$, as entradas do processo permanecem constantes durante vários períodos de amostragem, devido ao comportamento em degrau. Nesses casos, a decomposição SVD realizada na determinação das direções menos excitadas gera alguns valores singulares muito próximos de zero. As direções referentes a esses valores singulares podem ser pouco informativas, sendo necessário utilizar mais direções no problema de otimização do SPC. Por isso, para $T_{c d}$ elevado, deve-se utilizar um número maior de direções menos excitadas $\left(n_{p e}\right)$.

Estudo 2: Análise do nível de excitação $(\rho)$.

Os resultados estão mostrados nas Figura 5.8 e Figura 5.9. Como era de se esperar, as simulações indicam que o aumento do parâmetro $\rho$ favorece a excitação. Isso pode ser observado pelas amplitudes das oscilações das variáveis na Figura 5.8 e pelos valores de $J_{k}$ na Figura 5.9. Ainda nessa última figura, nota-se que o termo relativo à excitação cresce com o aumento do nível de excitação, devido à mudança na ordem de grandeza das diferenças entre a última ação de controle implementada e as direções menos excitadas. 

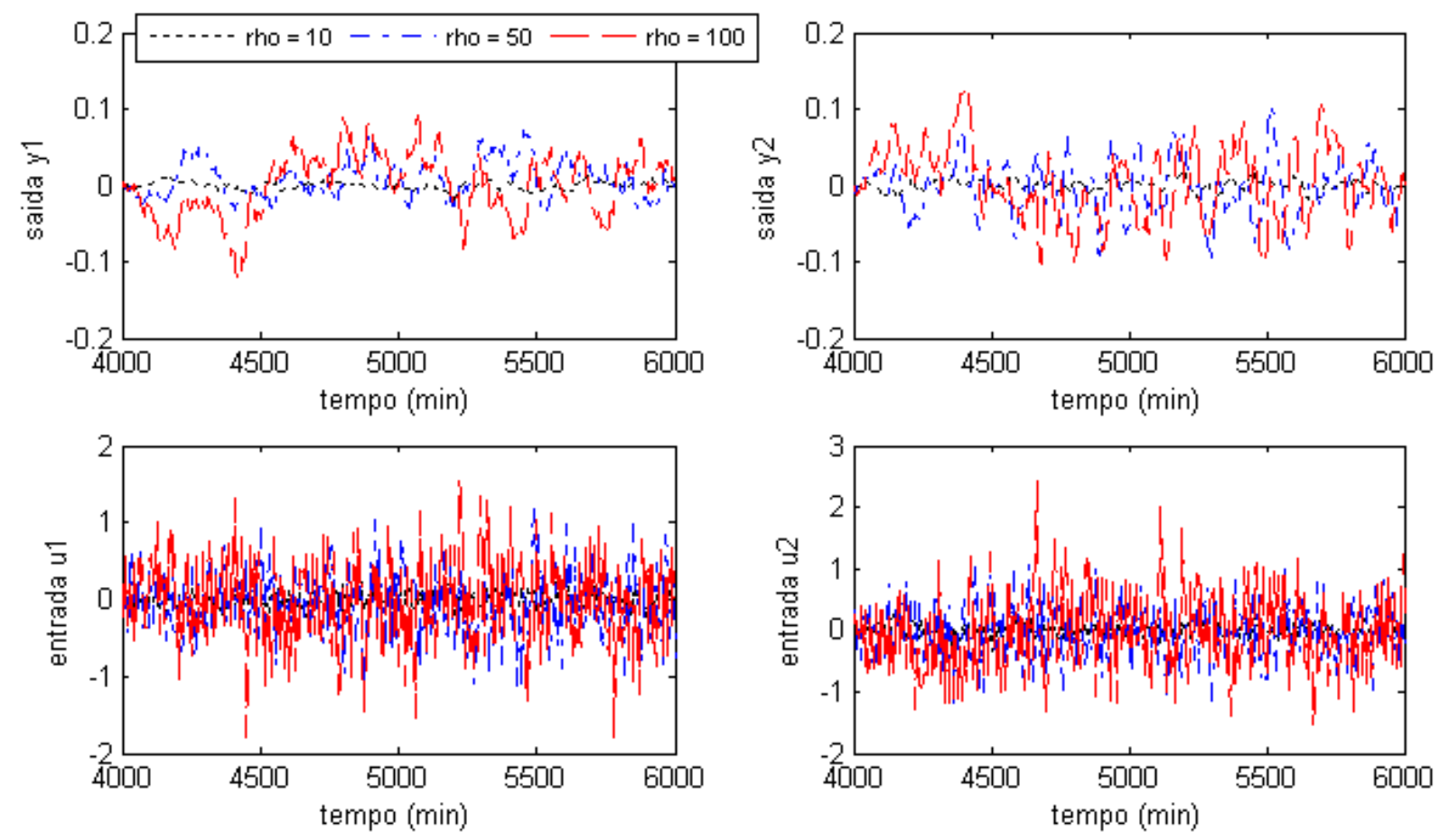

Figura 5.8 - Controlador SPC com excitação interna. Análise do nível de excitação.

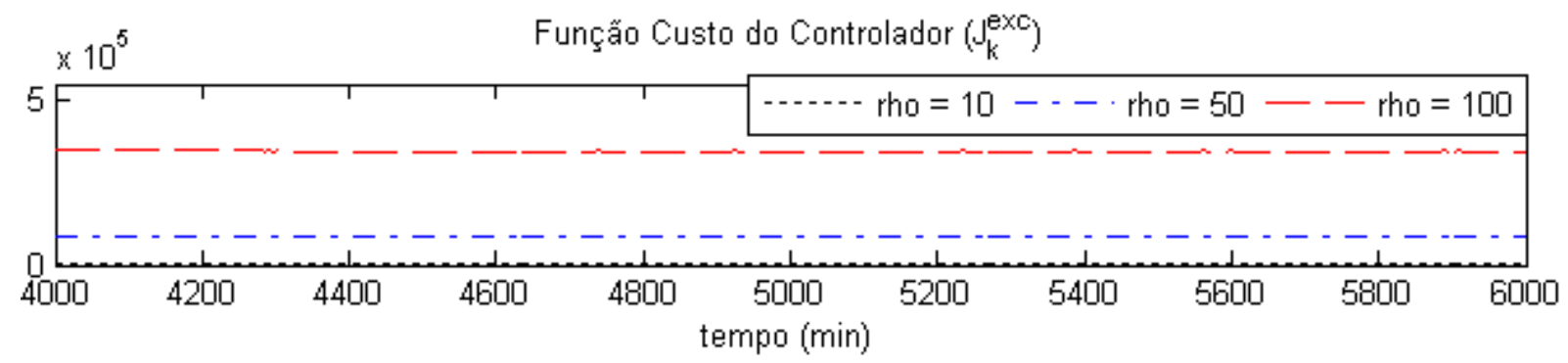

Parcela da Funçẫo Custo Referente ao Controle $\left(J_{k}\right)$
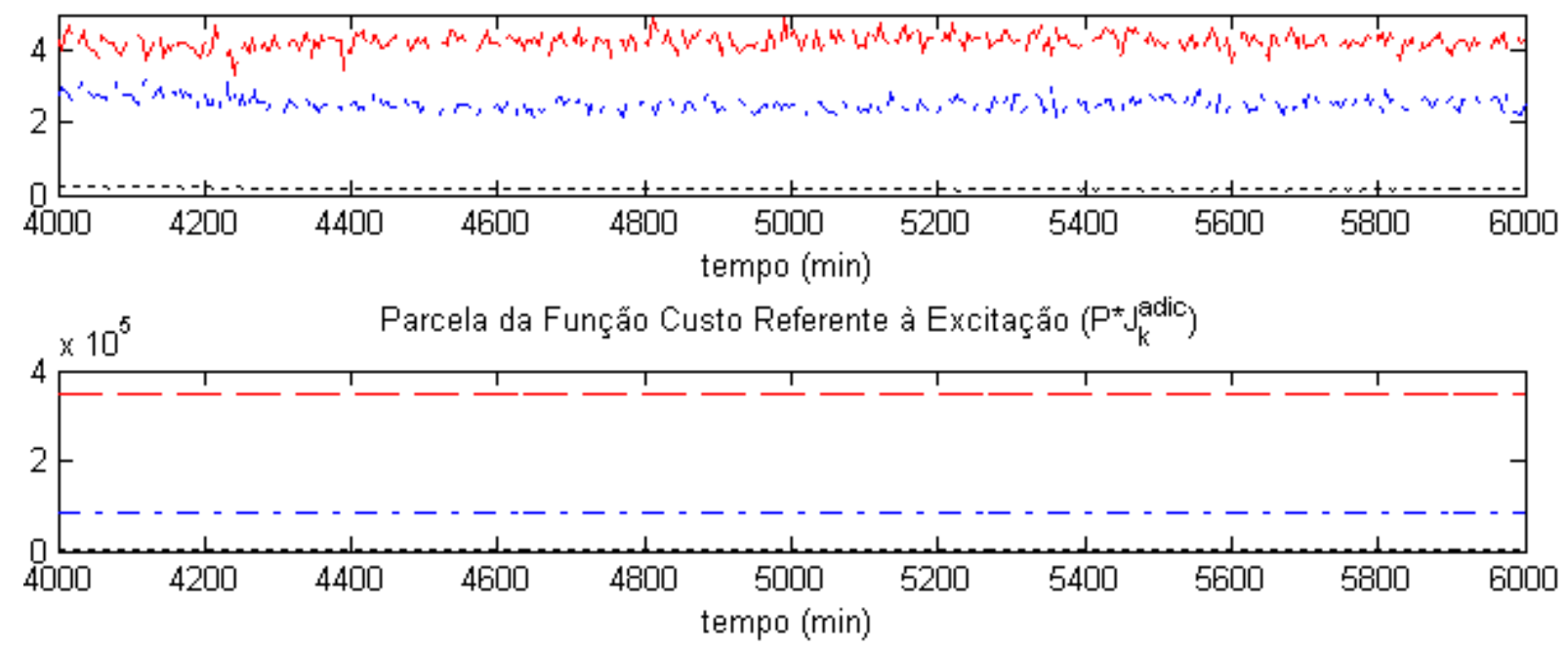

Figura 5.9 - Função objetivo do controlador SPC com excitação interna. Análise do nível de excitação. 
Foi testado o controlador no caso de $\rho=1$, porém as oscilações obtidas foram muito pequenas, na ordem de $10^{-3}$. Isso ocorreu porque a decomposição SVD realizada para o cálculo das direções menos excitadas gera matrizes normalizadas, resultando em direções com intensidades baixas. Assim, o parâmetro $\rho$ tem a função de corrigir a intensidade da excitação para um nível adequado.

Estudo 3: Análise do peso da excitação em relação ao controle $(P)$.

Os resultados estão apresentados nas Figura 5.10 e Figura 5.11. Diversas simulações mostraram que para determinada faixa de valores de $P$, um aumento desse parâmetro corresponde a um aumento na intensidade da excitação. Entretanto, observou-se a existência de valores de saturação, tanto um limite superior como um limite inferior, a partir dos quais não são observadas alterações significativas no grau de excitação. De fato, na Figura 5.11 nota-se que, para $P=0.01$, a parcela da função objetivo referente ao controle $\left(J_{k}\right)$ é menor. Para $P=1$ e $P=10$, os valores de $J_{k}$ são maiores, porém, entre estes dois casos, não há diferença.

O comportamento descrito acima se deve ao fato de que o parâmetro $P$ representa o peso do termo de excitação em relação ao termo de controle na função objetivo do problema de otimização. Para valores muito pequenos de $P$, o termo referente à excitação se torna desprezível, e o controle do processo prevalece totalmente. Por outro lado, valores de $P$ muito grandes tornam o termo referente ao controle desprezível, sendo que a máxima excitação será alcançada. Há uma faixa intermediária do peso $P$, na qual os dois termos da função custo possuem valores próximos e ambos os objetivos são levados em consideração na resolução do problema.

Na Figura 5.11, observa-se que o termo da função objetivo referente à excitação no caso de $P=10$ é em torno de dez vezes maior que esse termo no caso de $P=1$. Isso ocorre, pois o parâmetro $P$ é usado no problema de otimização como um fator multiplicativo no termo referente à excitação. 

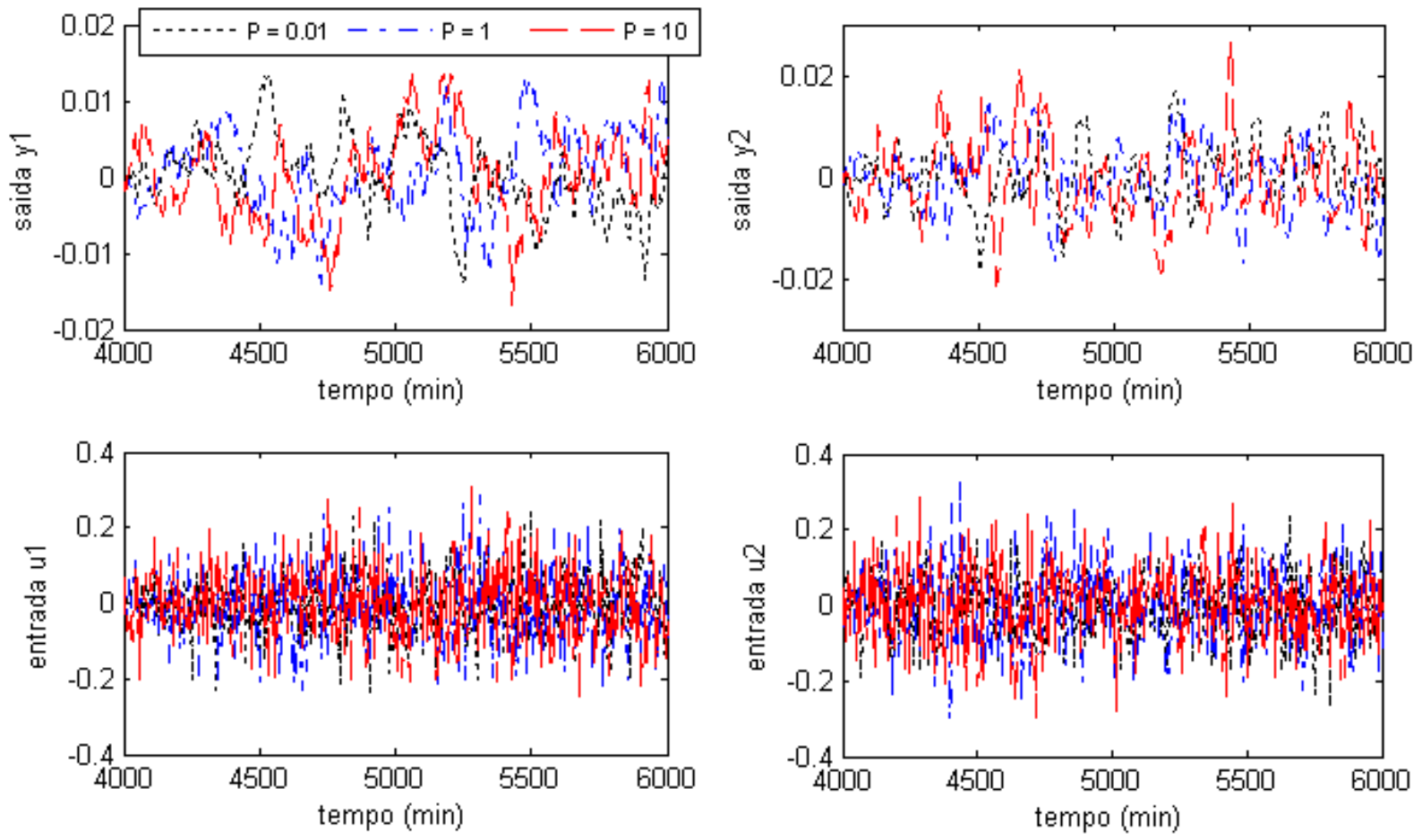

Figura 5.10 - Controlador SPC com excitação interna. Análise do peso da excitação em relação ao controle.

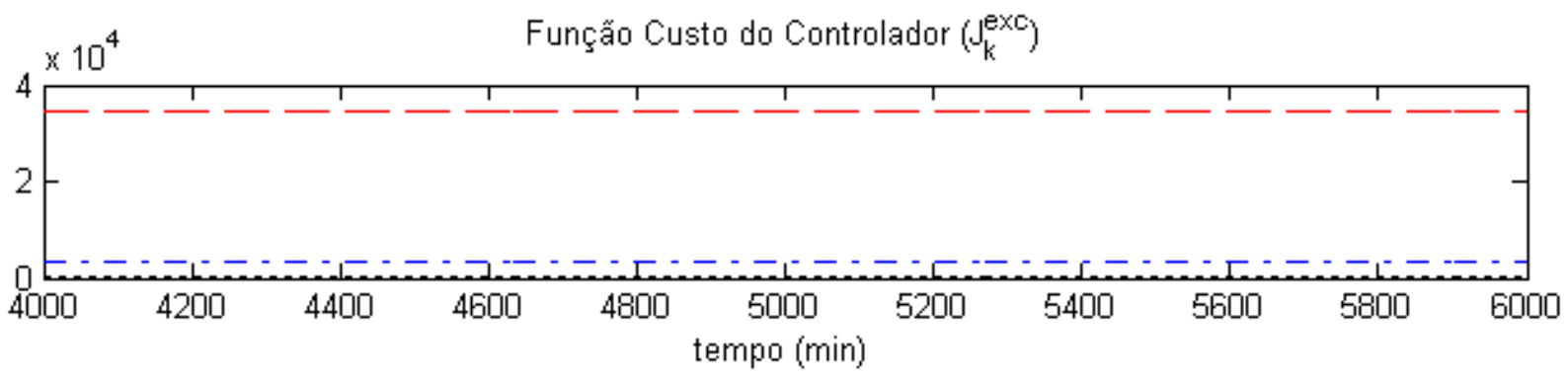

Parcela da Funçẫo Custo Referente ao Controle $\left(J_{k}\right)$
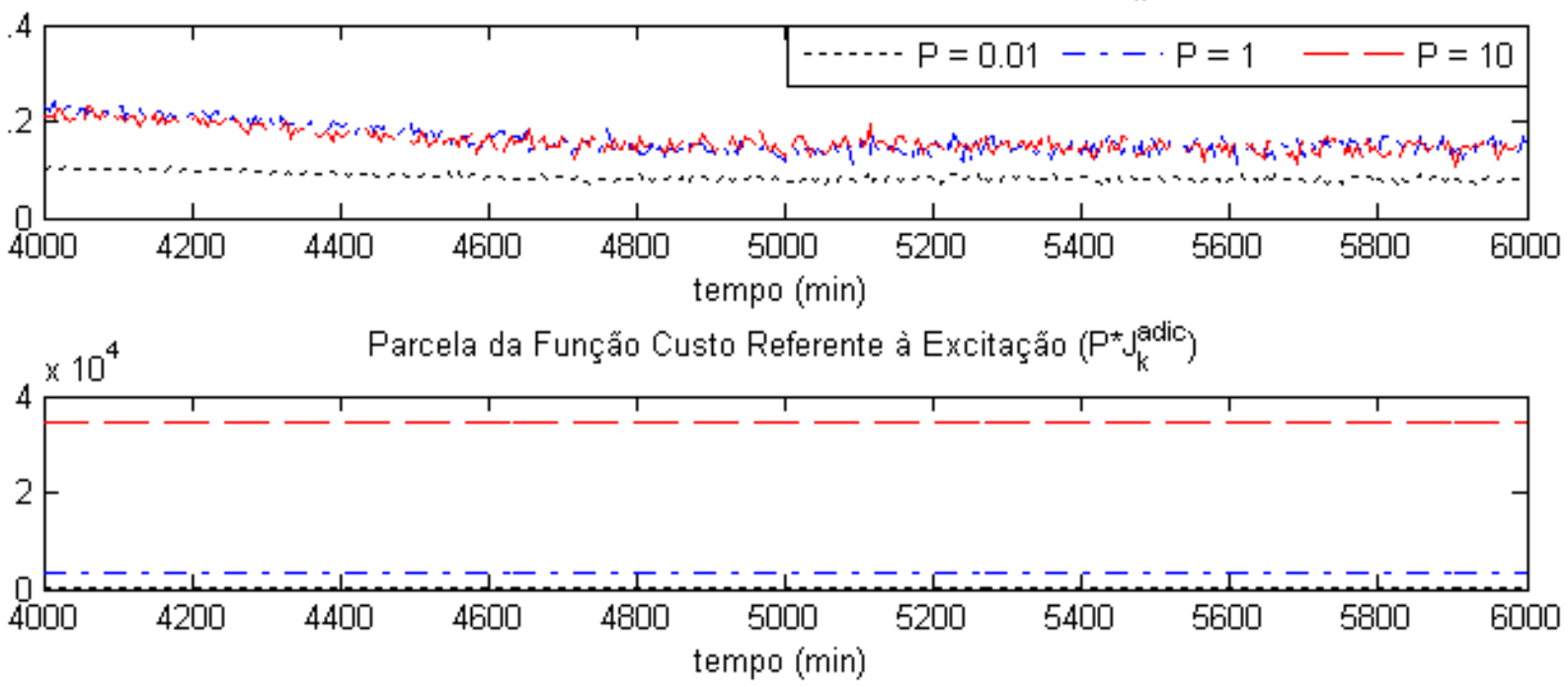

Figura 5.11 - Função objetivo do controlador SPC com excitação interna. Análise do peso da excitação em relação ao controle. 
Estudo 4: Análise do intervalo de cálculo das direções $\left(T_{c d}\right)$.
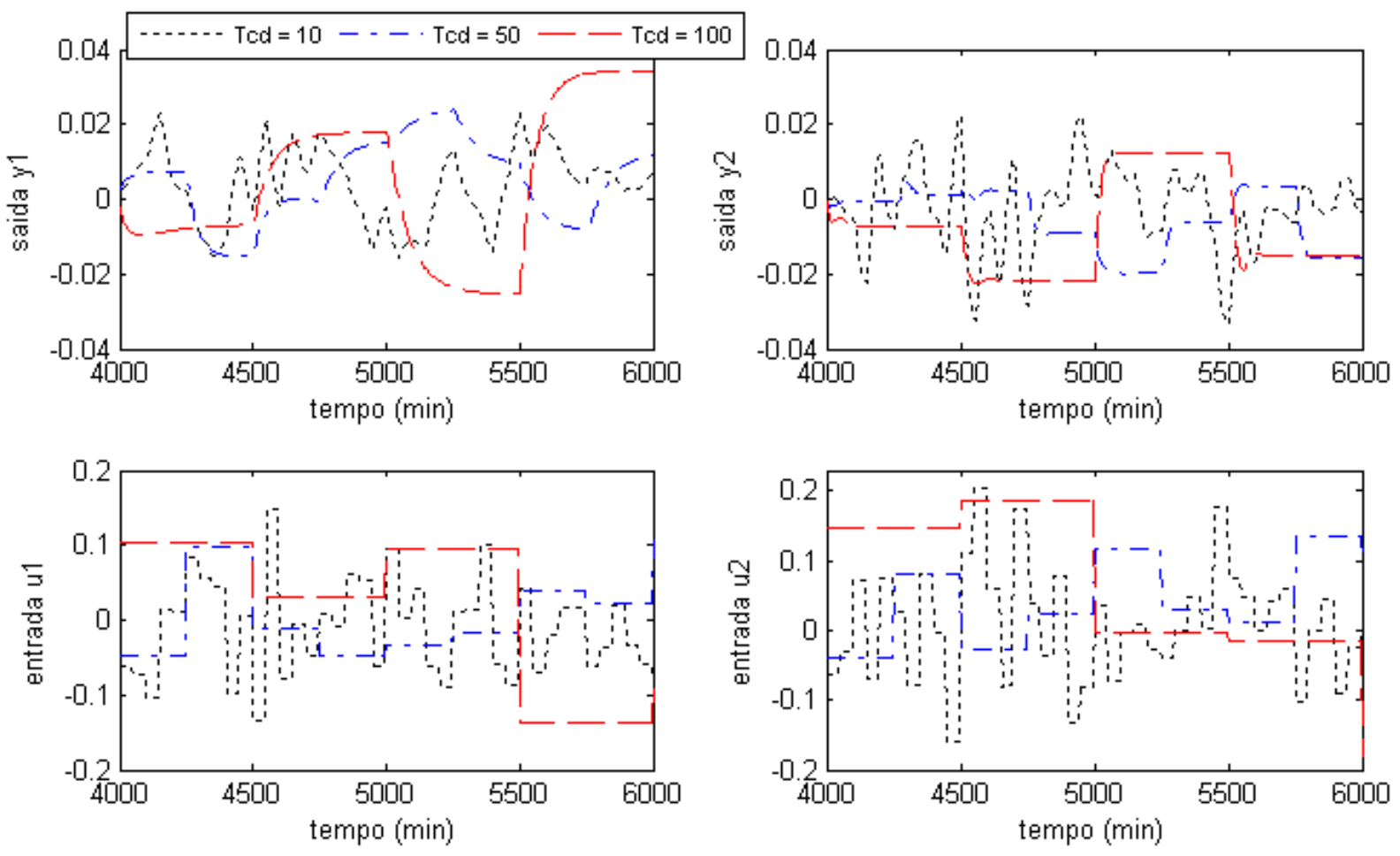

Figura 5.12 - Controlador SPC com excitação interna. Análise do intervalo de cálculo das direções.

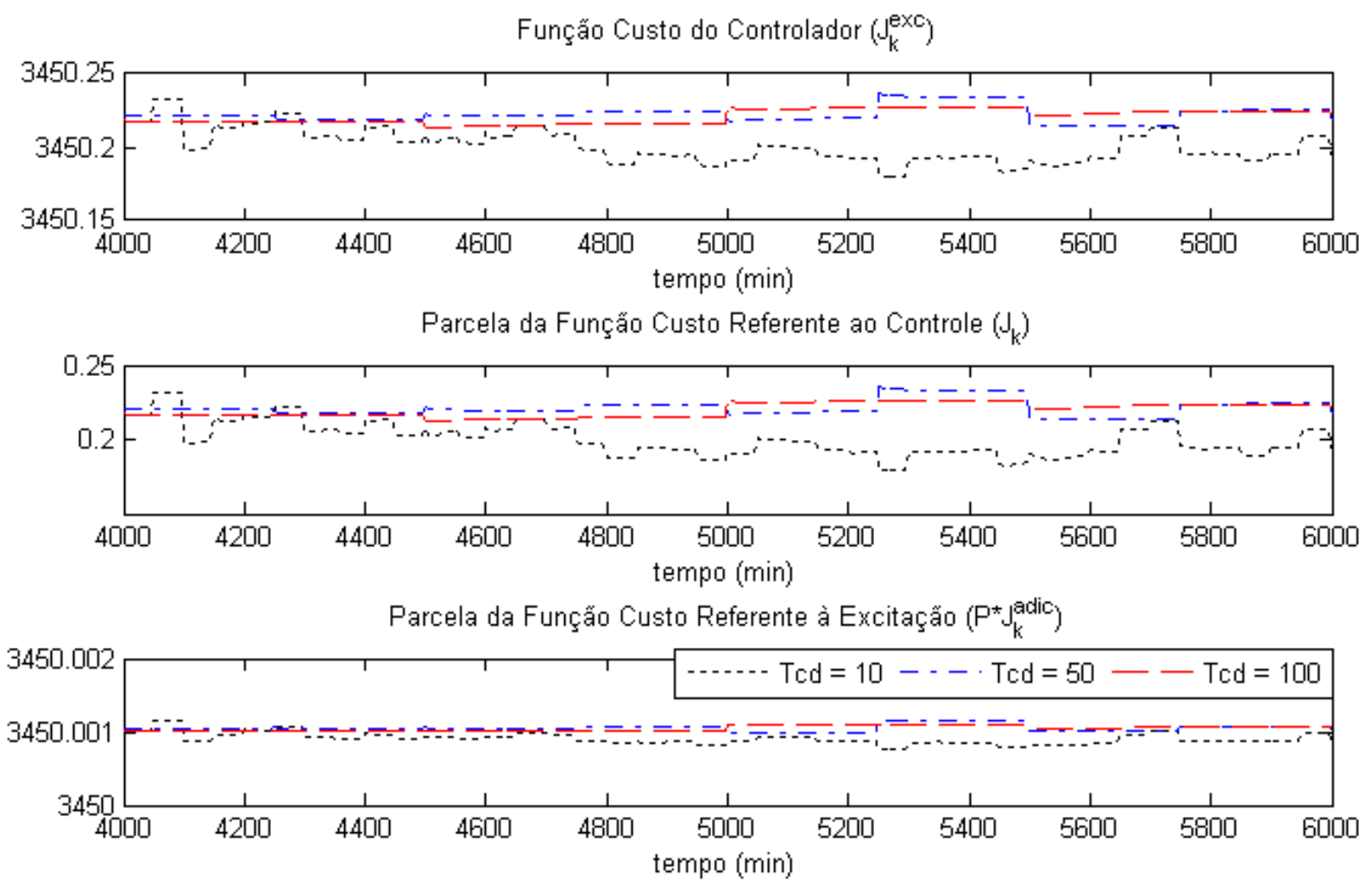

Figura 5.13 - Função objetivo do controlador SPC com excitação interna. Análise do intervalo de cálculo das direções. 
Nas Figura 5.12 e Figura 5.13, observa-se que, em todos os testes, o termo da função custo referente à excitação e as variáveis manipuladas possuem variações em forma de degraus de tamanhos equivalentes ao parâmetro $T_{c d}$. Entretanto, as variáveis controladas se comportam de maneiras diferentes em cada caso. Para $T_{c d}=10$, as oscilações nas saídas são contínuas, sendo que em nenhum momento seus valores permanecem constantes. No caso de $T_{c d}=50$, as variáveis controladas apresentam comportamento semelhante a respostas ao degrau, permitindo que a dinâmica lenta do sistema seja observada. Finalmente para $T_{c d}=100$, as variáveis controladas também se comportam como respostas ao degrau, mas seus valores permanecem fixos por períodos longos, indicando que esse intervalo de tempo está excessivamente elevado.

De maneira sucinta, a análise realizada permite concluir que o parâmetro de sintonia mais aconselhável para um ajuste fino na intensidade da excitação é o nível de excitação $(\rho)$. O peso da excitação $(P)$ deve ser usado somente para garantir que o termo da função custo referente à excitação seja pelo menos da mesma ordem de grandeza do termo referente ao controle. O número de direções menos excitadas $\left(n_{p e}\right)$ não deve ser muito elevado, de modo que apenas as direções pouco excitadas sejam consideradas. Por fim, o parâmetro $T_{c d}$ (intervalo de cálculo das direções) deve permitir que toda a dinâmica do processo seja observada, e ao mesmo tempo, deve evitar que as saídas do processo permaneçam constantes por períodos longos.

\subsubsection{Simulação do SPC com reidentificação em malha fechada}

Para testar o efeito da reidentificação do modelo de predição em subespaços a partir dos dados gerados pelo SPC com excitação interna, realizou-se uma simulação com o sistema da coluna debutanizadora, na qual o SPC com reidentificação do modelo foi comparado ao MPC com modelo constante. Considerou-se o caso de erro nos modelos nos controladores, sendo que a planta foi representada pelo Modelo C2 enquanto os modelos dos controladores foram representados pelo Modelo C1. $\mathrm{O}$ 
modelo de predição em subespaços usado no SPC foi obtido pela identificação com os dados do experimento $A$ (Tabela 3.4). O modelo do MPC foi obtido pela conversão da função de transferência do Modelo $C 1$ para o modelo em espaço de estados na forma incremental. Optou-se por utilizar o controlador SPC com cálculo do valor de referência, pois esse método reúne as vantagens dos outros dois métodos descritos nas seções 5.1 e 5.2. Para tornar a comparação mais coerente, a mesma sintonia foi utilizada para o SPC e o MPC. Optou-se por manter a sintonia base apresentada na Tabela 5.1 .

A simulação consistiu inicialmente no controle do processo em $y^{s p}=\left[\begin{array}{ll}1 & -1\end{array}\right]^{T}$, partindo-se da origem. A condição de excitação do SPC foi mantida desativada e apenas um pequeno ruído aleatório de variância 0.0001 foi adicionado à entrada do processo. No instante 3500 minutos, a excitação interna do controlador SPC foi ativada e permaneceu assim até o instante 7000 minutos. Nesse momento, 0 modelo de predição em subespaços foi reidentificado. Finalmente, em 7500 minutos, o controle foi alterado para $y^{s p}=\left[\begin{array}{ll}-1 & 1\end{array}\right]^{T}$. O resultado obtido está mostrado a seguir:
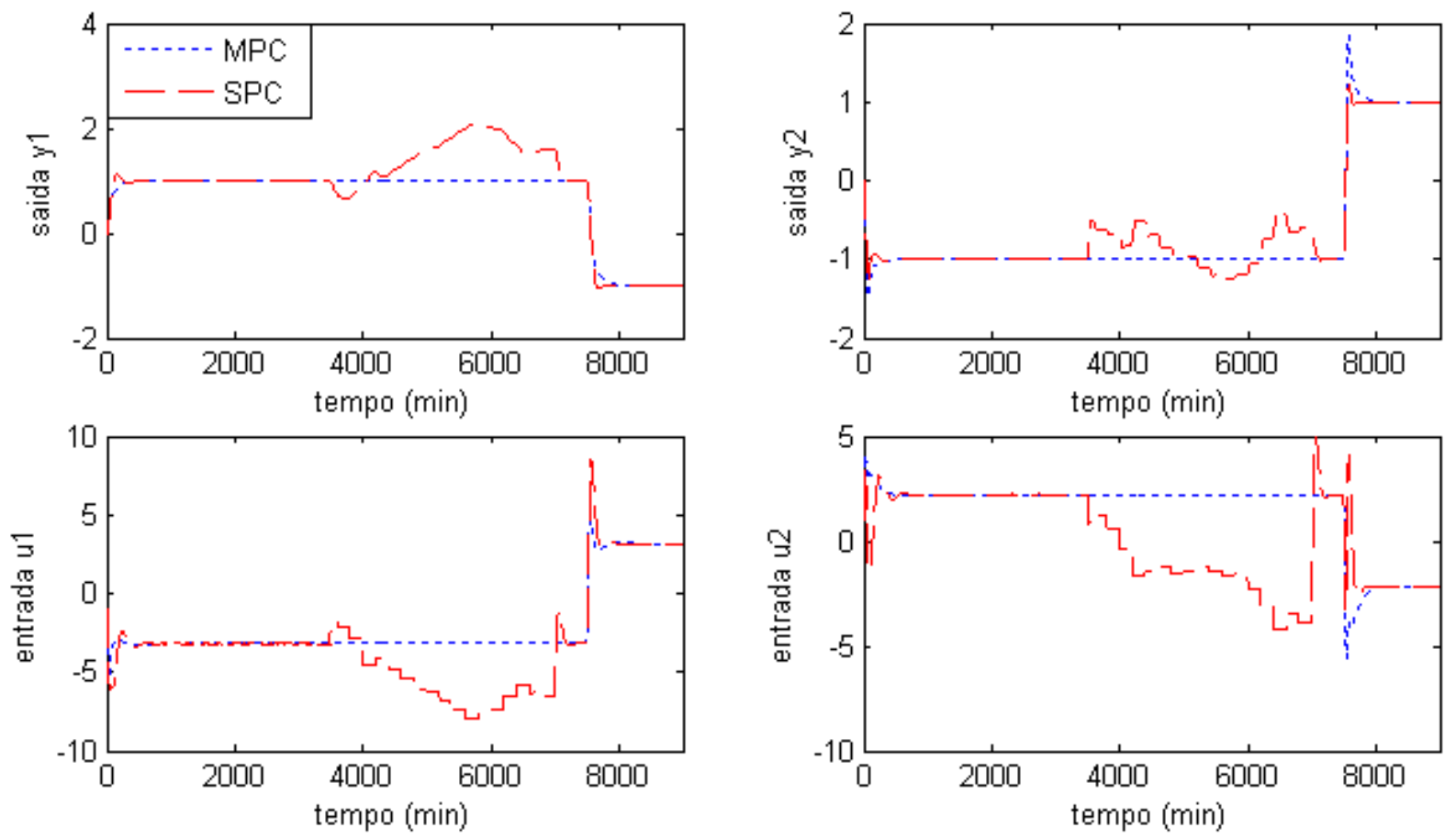

Figura 5.14 - Comparação entre MPC e SPC com reidentificação do modelo para o sistema da coluna debutanizadora. Visão Geral.

Para facilitar a análise da simulação, a Figura 5.14 foi dividida em três figuras que apresentam as variáveis do processo em intervalos de tempo menores: 

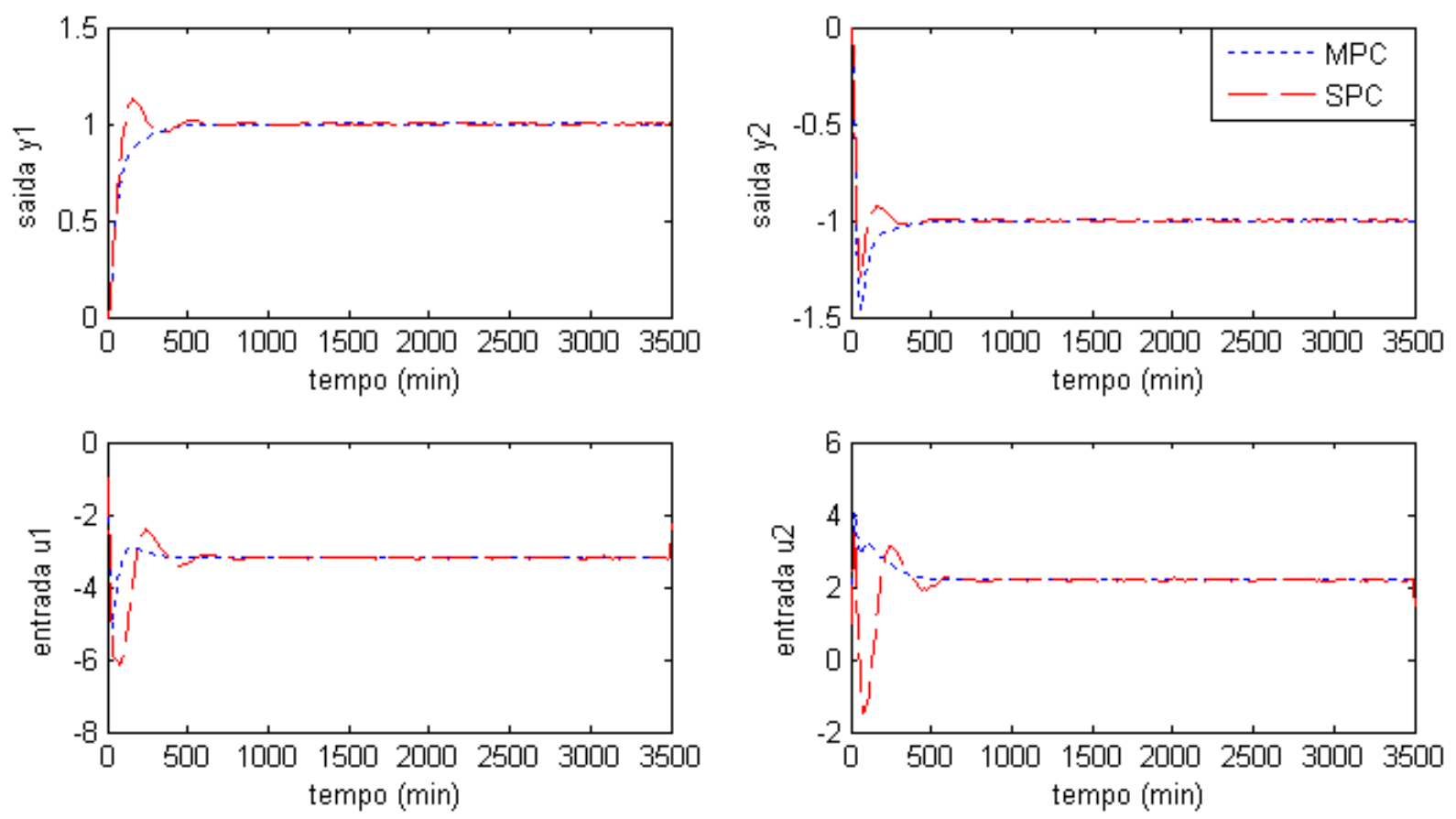

Figura 5.15 - Comparação entre MPC e SPC com reidentificação do modelo para o sistema da coluna debutanizadora. Ambos os controladores com modelo inicial (Período de 0 a 3500 minutos).

Analisando a Figura 5.15, percebe-se que no período em que os controladores possuem modelos iguais entre si, mas diferentes da planta real, o MPC obteve um desempenho superior, principalmente para a variável $y_{1}$. As variáveis controladas pelo SPC apresentaram oscilações antes de se estabilizarem em seus set-points.
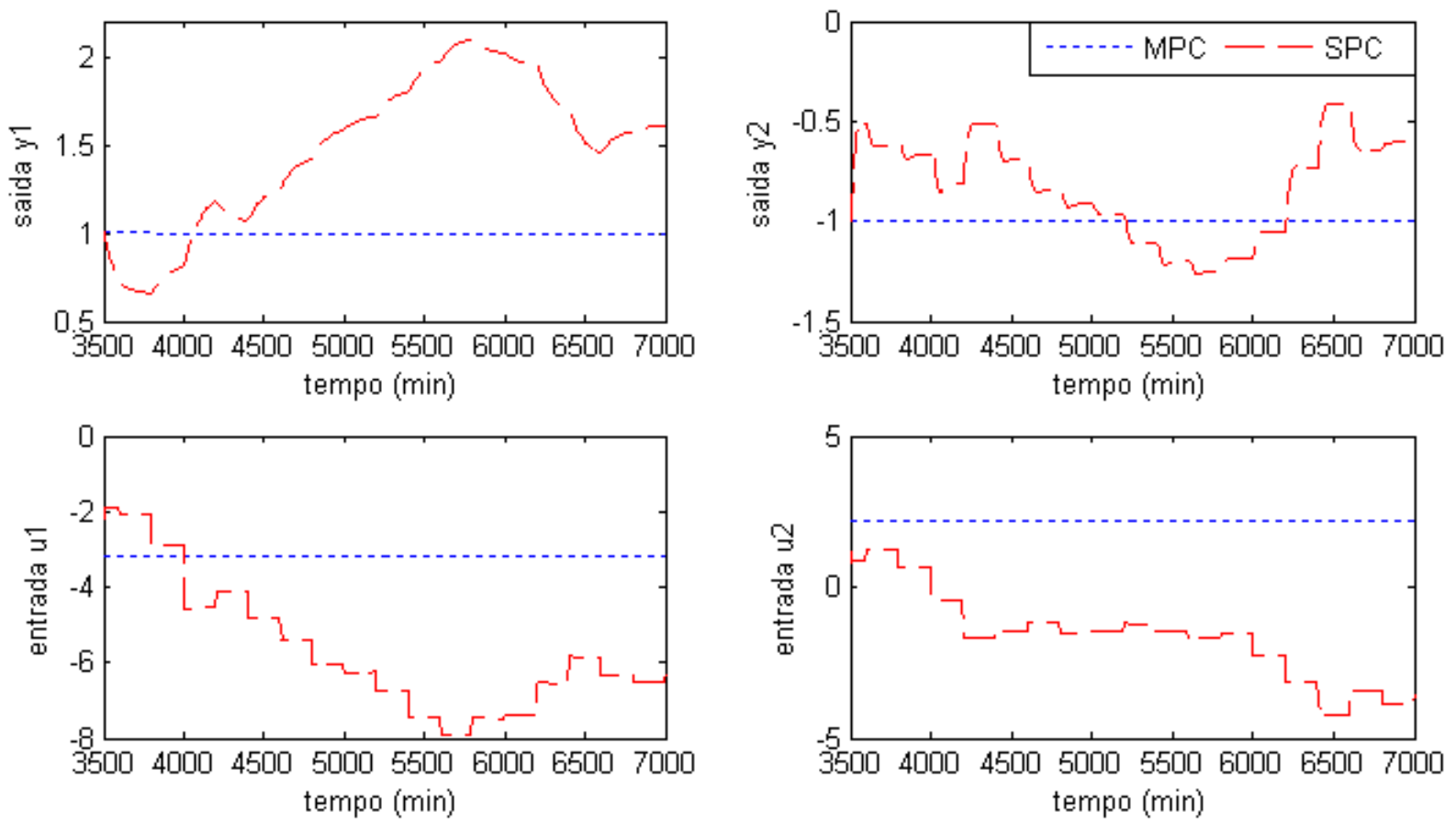

Figura 5.16 - Comparação entre MPC e SPC com reidentificação do modelo para o sistema da coluna debutanizadora. SPC com condição de excitação ativa (Período de 3500 a 7000 minutos). 
No intervalo de tempo de 3500 a 7000 minutos, as variáveis manipuladas do controlador MPC foram mantidas constantes, enquanto o controlador SPC iniciou a excitação do sistema. Observa-se, pela Figura 5.16, que nesse período as oscilações das variáveis controladas pelo SPC permaneceram aproximadamente em torno de seus set-points. Esse fato representa uma grande vantagem, pois permite um controle melhor da planta, mesmo na etapa de excitação.
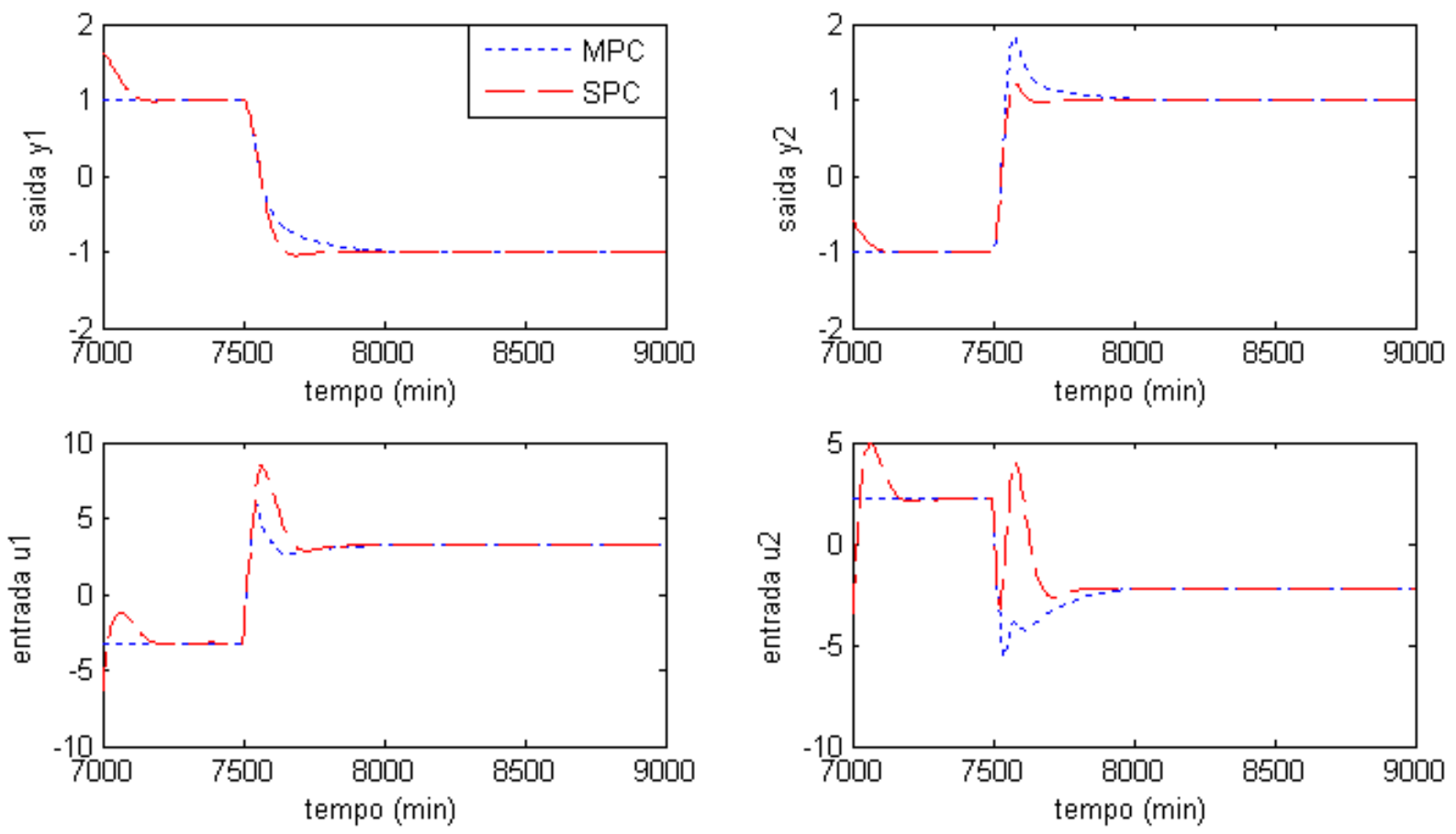

Figura 5.17 - Comparação entre MPC e SPC com reidentificação do modelo para o sistema da coluna debutanizadora. SPC com modelo reidentificado (Período de 7000 a 9000 minutos).

A Figura 5.17 mostra o período final da simulação, quando ocorre uma mudança de set-point nas variáveis controladas. O MPC permanece com o modelo inicial, enquanto o modelo usado no SPC sofreu reidentificação no instante 7000 minutos. Observa-se grande melhora no desempenho do SPC. Esse controlador passou, inclusive, a ter um comportamento melhor que o MPC. Isso mostra que o modelo reidentificado é mais próximo do sistema real do que o modelo usado inicialmente. $\mathrm{A}$ boa qualidade do novo modelo deve-se aos dados usados na identificação que, além de serem persistentemente excitantes, foram coletados na ausência de ruídos. Dessa maneira, fica comprovada a eficácia do método de excitação proposto. 


\section{APLICAÇÃO DO SPC PARA UM SISTEMA NÃO-LINEAR}

Sistemas não-lineares que utilizam controladores baseados em modelos lineares, como o MPC e o SPC, podem ser extremamente sensíveis ao ponto de operação. Uma mudança nos set-points das variáveis controladas pode alterar significativamente o comportamento do sistema, tornando o modelo usado pelo controlador distante do sistema real. Nesses casos, é preciso realizar uma reidentificação periódica do modelo.

Nos capítulos anteriores estudou-se a técnica de controle SPC para um sistema linear, sendo que os resultados obtidos foram bastante satisfatórios. Neste capítulo, o controlador SPC será aplicado para um sistema com dinâmica não-linear. Primeiramente, na seção 6.1, o sistema é apresentado. Na seção 6.2 é realizada uma análise do controlador SPC para o caso de erro no modelo. Finalmente, na seção 6.3, são apresentadas simulações do SPC com excitação interna que mostram a influência da reidentificação do modelo no desempenho do controlador.

\subsection{APRESENTAÇÃO DO SISTEMA}

\subsubsection{Descrição do modelo fenomenológico}

O sistema considerado consiste em um reator CSTR de polimerização de estireno. Considera-se que o reator é um volume perfeitamente agitado, no qual são adicionadas correntes de iniciador, solvente e monômero. O iniciador usado é o 2,2azo-bis-iso-butironitrila (AIBN) dissolvido em benzeno; o monômero é estireno puro; e o solvente é benzeno puro. Para o controle da temperatura de operação, o sistema é envolto por uma camisa de refrigeração. A Figura 6.1 ilustra esquematicamente 0 reator CSTR. 


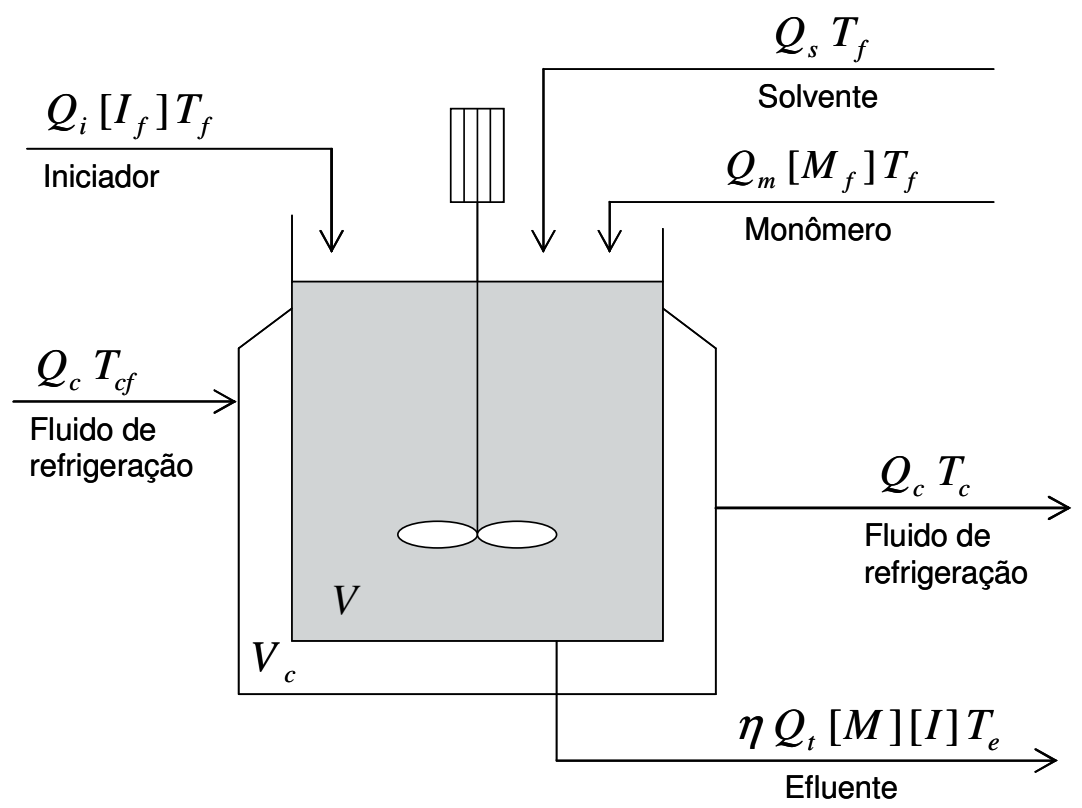

Figura 6.1 - Representação esquemática do reator CSTR de polimerização de estireno.

A cinética do mecanismo de homopolimerização pode ser descrita pelos seguintes passos (SOTOMAYOR; ODLOAK; GIUDICI, 2007):

$$
\begin{array}{lll}
I \stackrel{f_{i}, k_{d}}{\rightarrow} 2 R & & \text { (decomposição do iniciador) } \\
M+R & \stackrel{k_{i}}{\rightarrow} P_{1} & \text { (início da cadeia) } \\
P_{n}+M & \stackrel{k_{p}}{\rightarrow} P_{n+1} & \text { (propagação) } \\
P_{n}+P_{m} \stackrel{k_{l d}}{\rightarrow} T_{n}+T_{m} & \text { (terminação por desproporcionamento) } \\
P_{n}+P_{m} \stackrel{k_{c c}}{\rightarrow} T_{n+m} & \text { (terminação por combinação) }
\end{array}
$$

sendo $I$ O iniciador, $R$ ○ radical produzido pela decomposição do iniciador, $M \circ$ monômero, $P$ a cadeia crescente de polímero e $T$ a cadeia de polímero terminada. Com base em equações cinéticas, balanços de massa e balanços de energia, Hidalgo e Brosilow (1990) apresentaram o seguinte modelo fenomenológico para o reator de polimerização:

$$
\frac{d[I]}{d t}=\frac{\left(Q_{i}\left[I_{f}\right]-Q_{t}[I]\right)}{V}-k_{d}[I]
$$




$$
\begin{aligned}
& \frac{d[M]}{d t}=\frac{\left(Q_{m}\left[M_{f}\right]-Q_{t}[M]\right)}{V}-k_{p}[M][P] \\
& \frac{d T_{e}}{d t}=\frac{Q_{t}\left(T_{f}-T_{e}\right)}{V}+\frac{\left(-\Delta H_{r}\right)}{\rho C_{p}} k_{p}[M][P]-\frac{h A}{\rho C_{p} V}\left(T_{e}-T_{c}\right) \\
& \frac{d T_{c}}{d t}=\frac{Q_{c}\left(T_{c f}-T_{c}\right)}{V_{c}}+\frac{h A}{\rho_{c} C_{p c} V_{c}}\left(T_{e}-T_{c}\right)
\end{aligned}
$$

sendo:

$$
\begin{aligned}
& {[P]=\left[\frac{2 f_{i} k_{d}[I]}{k_{t}}\right]^{0.5}} \\
& k_{j}=A_{j} \exp \left(\frac{-E_{j}}{T_{e}}\right), \quad j=d, p, t \\
& Q_{t}=Q_{i}+Q_{s}+Q_{m}
\end{aligned}
$$

Esse modelo foi estendido por Maner et al. (1996) de modo a possibilitar o cálculo do peso molecular médio do polímero $\left(\bar{M}_{w}\right)$. Foram adicionadas duas equações de momento:

$$
\begin{aligned}
& \frac{d D_{0}}{d t}=0.5 k_{t}[P]^{2}-\frac{Q_{t} D_{0}}{V} \\
& \frac{d D_{1}}{d t}=M_{m} k_{p}[M][P]-\frac{Q_{t} D_{1}}{V}
\end{aligned}
$$

sendo

$$
\bar{M}_{w}=\frac{D_{1}}{D_{0}}
$$

Como não é possível medir $\bar{M}_{w}$ diretamente da planta, Sotomayor; Odloak e Giudici (2007) propuseram utilizar a viscosidade intrínseca $(\eta)$, que pode ser medida por um viscosímetro on-line. Essa viscosidade é relacionada com o peso molecular médio pela seguinte expressão:

$$
\eta=0.0012\left(\bar{M}_{w}\right)^{0.71}
$$


Considerou-se que a vazão de solvente é determinada pela seguinte relação com as vazões de monômero e de iniciador:

$$
Q_{s}=1.5 Q_{m}-Q_{i}
$$

A descrição de cada variável é apresentada nas Tabela 6.1 e Tabela 6.2. Além disso, essas tabelas mostram, respectivamente, as condições operacionais e os valores dos parâmetros do modelo no estado estacionário, ambos retirados de Maner et al. (1996).

Tabela 6.1 - Condições operacionais do reator de polimerização no estado estacionário.

\begin{tabular}{|c|c|c|}
\hline Variável & Símbolo & Valor \\
\hline Vazão de solvente & $Q_{s}$ & 459 L.h $^{-1}$ \\
\hline Vazão de monômero & $Q_{m}$ & 378 L.h.' \\
\hline Volume do reator & $V$ & $3000 \mathrm{~L}$ \\
\hline Volume da camisa de refrigeração & $V_{c}$ & $3312.4 \mathrm{~L}$ \\
\hline Temperatura da alimentação do reator & $T_{f}$ & $330 \mathrm{~K}$ \\
\hline Temperatura de entrada do fluido de refrigeração & $T_{c f}$ & $295 \mathrm{~K}$ \\
\hline Concentração do iniciador na alimentação & {$\left[I_{f}\right]$} & $0.5888 \mathrm{~mol}^{-\mathrm{L}^{-1}}$ \\
\hline Concentração do monômero na alimentação & {$\left[M_{f}\right]$} & $8.6981 \mathrm{~mol}^{-\mathrm{L}^{-1}}$ \\
\hline Concentração do iniciador no reator & {$[I]$} & $6.6832 \times 10^{-2} \mathrm{~mol}^{-\mathrm{L}^{-1}}$ \\
\hline Concentração do monômero no reator & {$[M]$} & $3.3245 \mathrm{~mol}^{-\mathrm{L}^{-1}}$ \\
\hline Temperatura final do fluido de refrigeração & $T_{c}$ & $305.17 \mathrm{~K}$ \\
\hline Concentração molar da cadeia de polímero terminada & $D_{0}$ & $2.7547 \times 10^{-4} \mathrm{~mol}^{-\mathrm{L}^{-1}}$ \\
\hline Concentração mássica da cadeia de polímero terminada & $D_{1}$ & $16.110 \mathrm{~g} \cdot \mathrm{L}^{-1}$ \\
\hline Vazão do iniciador & $Q_{i}$ & 108 L.h $^{-1}$ \\
\hline Vazão do fluido de refrigeração & $Q_{c}$ & 471.6 L.h $^{-1}$ \\
\hline Viscosidade intrínseca & $\eta$ & $2.9091{\mathrm{~L} . \mathrm{g}^{-1}}^{-1}$ \\
\hline Temperatura no reator & $T_{e}$ & $323.56 \mathrm{~K}$ \\
\hline
\end{tabular}


Tabela 6.2 - Parâmetros do processo de polimerização no estado estacionário.

\begin{tabular}{|c|c|c|}
\hline Parâmetro do modelo & Símbolo & Valor \\
\hline Fator pré-exponencial para a decomposição do iniciador & $A_{d}$ & $2.142 \times 10^{17} \mathrm{~h}^{-1}$ \\
\hline Energia de ativação para a decomposição do iniciador & $E_{d}$ & $14897 \mathrm{~K}$ \\
\hline Fator pré-exponencial para a reação de propagação & $A_{P}$ & $3.816 \times 10^{10}$ L. $\mathrm{mol}^{-1} \cdot \mathrm{h}^{-1}$ \\
\hline Energia de ativação para a reação de propagação & $E_{P}$ & $3557 \mathrm{~K}$ \\
\hline Fator pré-exponencial para a reação de terminação & $A_{t}$ & $4.50 \times 10^{12}$ L. $\mathrm{mol}^{-1} \cdot \mathrm{h}^{-1}$ \\
\hline Energia de ativação para a reação de terminação & $E_{t}$ & $843 \mathrm{~K}$ \\
\hline Eficiência do iniciador & $f_{i}$ & 0.6 \\
\hline Entalpia da reação de polimerização & $-\Delta H_{r}$ & $16700 \mathrm{cal} . \mathrm{mol}^{-1}$ \\
\hline Peso molecular do monômero & $M_{m}$ & $104.14 \mathrm{~g} \cdot \mathrm{mol}^{-1}$ \\
\hline Coeficiente global de transferência de calor & $h A$ & $2.52 \times 10^{5} \mathrm{cal} . \mathrm{K}^{-1} \cdot \mathrm{h}^{-1}$ \\
\hline Capacidade calorífica do fluido no reator & $\rho C_{p}$ & 360 cal. $\mathrm{K}^{-1} \cdot \mathrm{L}^{-1}$ \\
\hline Capacidade calorífica do fluido na camisa & $\rho_{c} C_{p c}$ & 966.3 cal. $\mathrm{K}^{-1} \cdot \mathrm{L}^{-1}$ \\
\hline
\end{tabular}

Para o controle do processo, foram consideradas duas variáveis manipuladas e duas variáveis controladas, conforme Tabela 6.3 e Tabela 6.4, respectivamente.

Tabela 6.3 - Variáveis manipuladas do sistema do reator de polimerização.

\begin{tabular}{ccc}
\hline Variável & \multicolumn{1}{c}{ Descrição } & Unidade \\
\hline$u_{1}$ & Vazão de alimentação do iniciador $\left(Q_{i}\right)$ & L.h $^{-1}$ \\
\hline$u_{2}$ & Vazão do fluido de refrigeração $\left(Q_{c}\right)$ & L. $^{-1}$ \\
\hline
\end{tabular}

Tabela 6.4 - Variáveis controladas do sistema do reator de polimerização.

\begin{tabular}{clc}
\hline Variável & \multicolumn{1}{c}{ Descrição } & Unidade \\
\hline$y_{1}$ & Viscosidade intrínseca $(\eta)$ & ${\mathrm{L} . g^{-1}}^{-1}$ \\
\hline$y_{2}$ & Temperatura no reator $\left(T_{e}\right)$ & $\mathrm{K}$ \\
\hline
\end{tabular}

Em todas as simulações presentes neste capítulo, a planta será representada pelo modelo fenomenológico do reator de polimerização definido em (6.1)-(6.8). As equações diferenciais serão integradas pelo método de Euler, com um passo de integração de 0.01 horas. $O$ período de amostragem será de 0.2 horas. 


\subsubsection{Linearização do sistema}

Para que seja possível aplicar os controladores MPC e SPC no sistema do reator de polimerização, primeiramente, é necessário encontrar um modelo linear que represente o processo de forma satisfatória. Com esse intuito, uma simulação foi realizada, na qual, partindo-se do estado estacionário definido na Tabela 6.1, um degrau unitário foi inserido no sistema em malha aberta. A resposta obtida foi então aproximada por um modelo linear de primeira ordem com tempo morto. O modelo resultante está mostrado abaixo:

Modelo R1: $\quad G(s)=\left[\begin{array}{ll}\frac{-0.013}{8 s+1} e^{-1.0 s} & \frac{0.001}{10 s+1} e^{-1.8 s} \\ \frac{0.037}{8 s+1} e^{-0.5 s} & \frac{-0.012}{11 s+1} e^{-0.3 s}\end{array}\right]$

No modelo acima a unidade de tempo utilizada é horas. A Figura 6.2 compara a resposta ao degrau do sistema real com a resposta ao degrau do modelo linear:
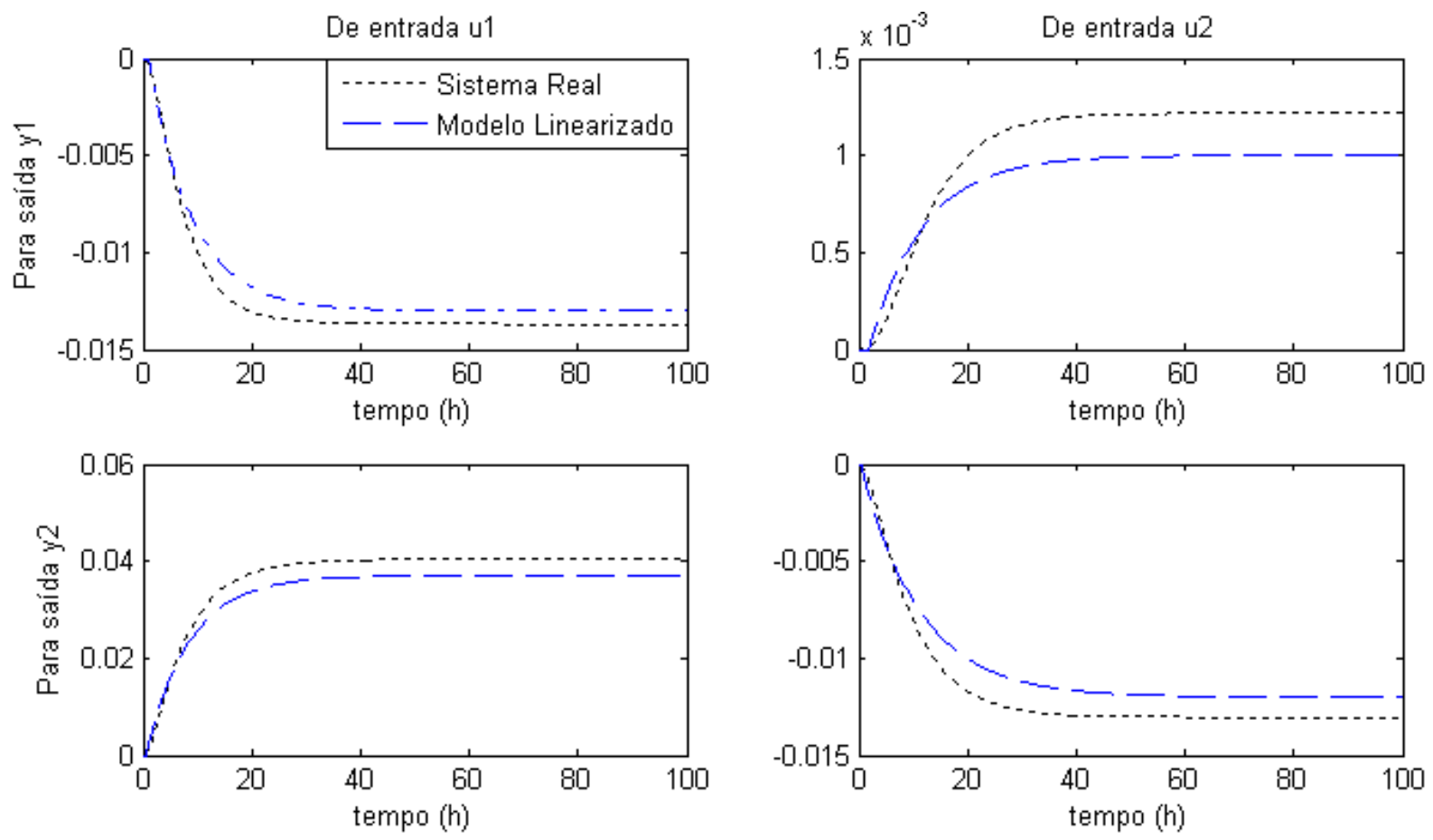

Figura 6.2 - Comparação entre as respostas ao degrau do sistema do reator de polimerização e do modelo linear. 
Observa-se que o modelo linear não representa o processo rigorosamente. Os ganhos estáticos do modelo são um pouco diferentes dos ganhos do sistema real, porém a qualidade desse modelo é suficiente para o controle preditivo do processo. Propositalmente estimou-se um modelo de qualidade regular, pois se deseja, posteriormente, estudar a reidentificação em malha fechada do sistema.

\subsubsection{Resposta do processo em diferentes pontos de operação}

Na seção 6.3, será estudada a situação em que a planta, inicialmente controlada no estado estacionário apresentado na Tabela 6.2, sofre uma mudança no seu ponto de operação, correspondente a um decréscimo de $10 \%$ no parâmetro $A_{t}$ (fator préexponencial para a reação de terminação). Essa mudança provoca uma alteração no comportamento do sistema. Para analisar essa alteração foram comparadas as respostas ao degrau do sistema no estado estacionário original e no estado estacionário referente à $A_{t} \quad 10 \%$ menor. A Figura 6.3 mostra o resultado obtido.
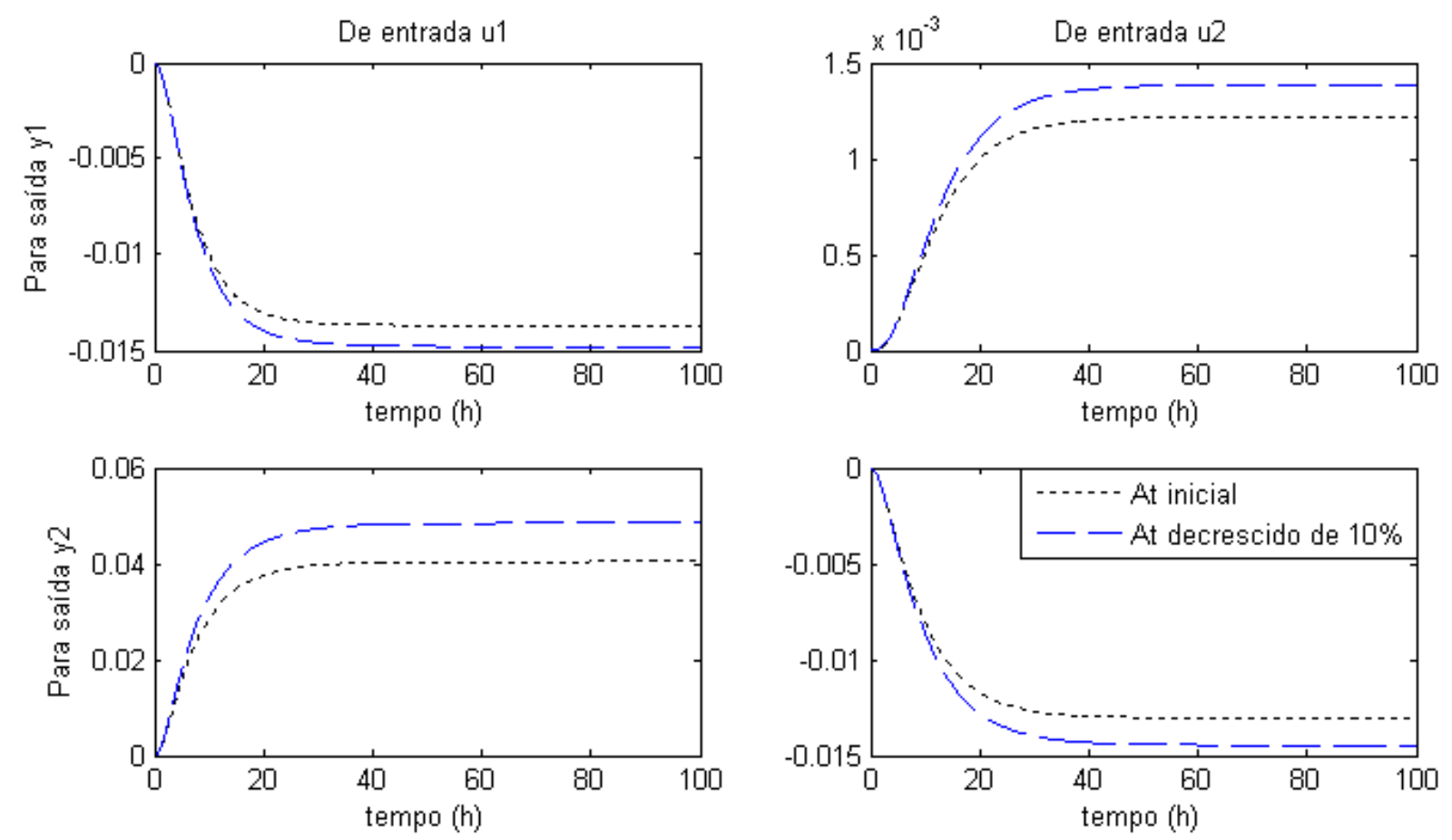

Figura 6.3 - Comparação entre as respostas ao degrau do reator de polimerização em diferentes pontos de operação. 
Observa-se, pela Figura 6.3, que no novo ponto de operação os ganhos do sistema são maiores e, portanto, o processo responde de forma diferente do sistema no estado estacionário original quando submetido ao mesmo sinal de entrada. Isso provoca uma degradação dos modelos dos controladores preditivos, que passam a não corresponder ao processo real com a mesma precisão.

\subsection{SINTONIA DO CONTROLADOR SPC}

Será estudado o cenário em que, inicialmente, não estão disponíveis dados amostrados do sistema real, sendo apenas conhecido um modelo aproximado do processo (Modelo R1). Para o MPC, obtém-se o modelo em espaço de estados a partir da função de transferência do Modelo R1. Uma vez feito isso, o modelo de predição em subespaços usado no SPC poderia ser calculado pelas equações (4.10)-(4.11). Porém, optou-se por determinar esse modelo via identificação. Com essa finalidade, realizou-se uma simulação na qual as entradas do Modelo $R 1$ foram excitadas por um sinal do tipo PRBS em malha aberta. Os dados dessa simulação foram então utilizados no cálculo do modelo de predição em subespaços.

$\mathrm{Na}$ identificação do modelo de predição é necessário definir o horizonte de dados passados $M$, que, conforme estudado na seção 4.3.1, influi no desempenho do controlador SPC. No intuito de analisar essa influência no caso do sistema do reator de polimerização foram realizados diversos testes. Simulou-se o controle desse processo em $y^{s p}=\left[\begin{array}{ll}0.1 & -0.1\end{array}\right]^{T}$ (valores relativos ao estado estacionário), partindo-se da origem. $O$ valor de $M$ foi alterado enquanto todos os outros parâmetros de sintonia foram mantidos constantes, em uma sintonia base, que foi definida por meio de tentativa e erro e é apresentada na Tabela 6.5.

O resultado das simulações está apresentado na Figura 6.4. Para facilitar a visualização, foram impressos os valores das variáveis relativos ao estado estacionário (Tabela 6.1). Esse padrão será mantido ao longo deste capítulo. 

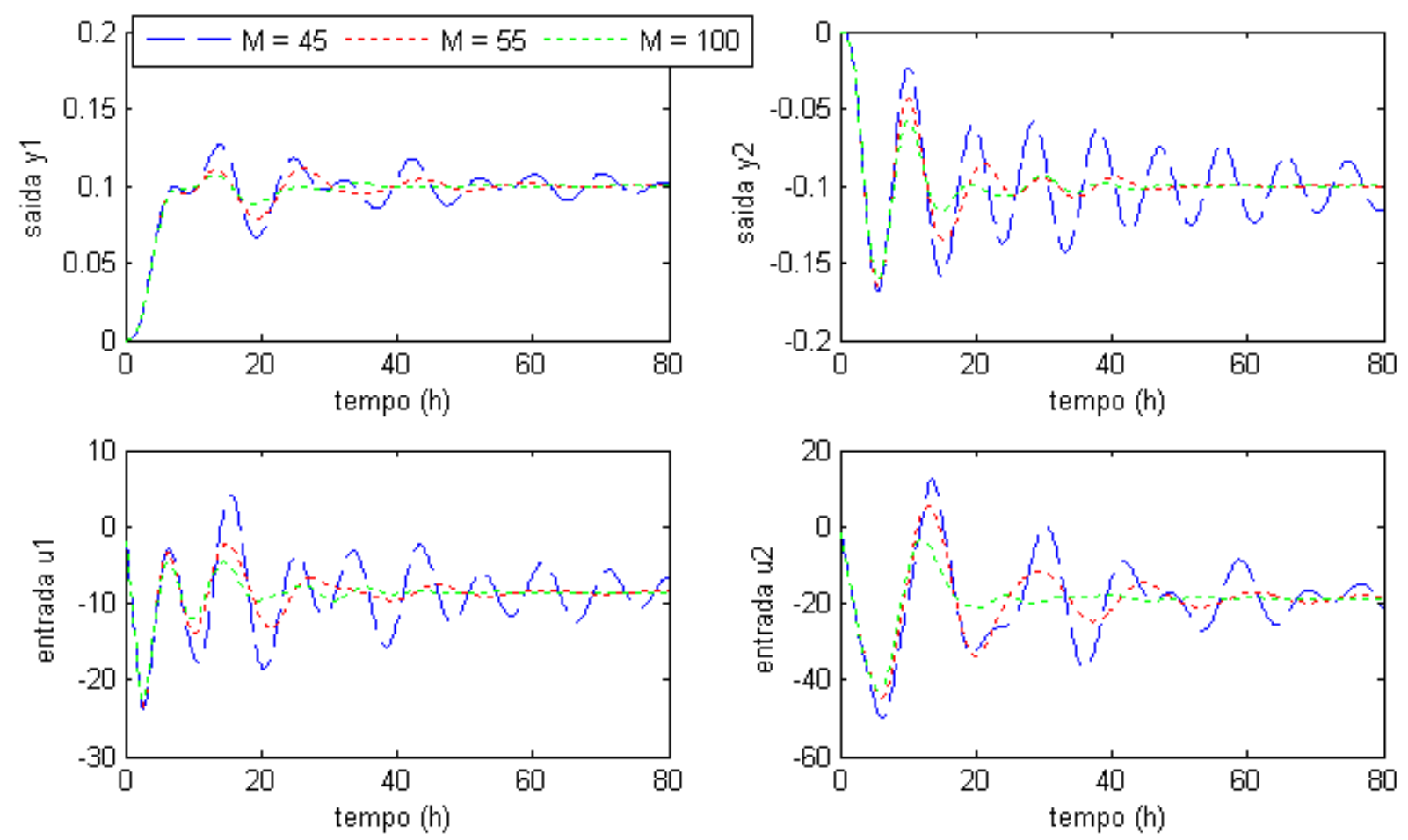

Figura 6.4 - Influência do horizonte de dados passados no SPC para o sistema do reator de polimerização.

Através da Figura 6.4, é possível observar que o desempenho do controlador melhora com o aumento do horizonte $M$. Isso ocorre, pois, os dados usados na identificação do modelo de predição em subespaços são livres de ruídos e gerados por um sistema linear (Modelo R1). Nota-se que para $M=45$ as variáveis do processo oscilam muito e as controladas não chegam a se estabilizar em seus setpoints durante o período de simulação (80 horas). Além disso, comparando-se a figura acima com a Figura 4.6, pode-se concluir que o parâmetro $M$ exerce uma influência muito maior no controle de um sistema não-linear do que no controle de um sistema linear.

Quanto maior o valor de $M$, maior o esforço computacional necessário para resolver o problema de otimização do SPC, principalmente quando a condição de excitação se encontra ativa. Além disso, o número de dados necessários na identificação do modelo é proporcional a esse parâmetro, devido ao requisito de que o número de colunas das matrizes de blocos de Hankel deve ser muito maior que o número de linhas, determinado por $M$. Um último ponto refere-se à precisão do modelo quando os dados são coletados na presença de distúrbios ou quando são obtidos a partir de um sistema não-linear. Nesses casos, um horizonte $M$ grande pode introduzir 
grandes erros de predição. Portanto, deve-se escolher o menor valor de $M$ que permita um controle satisfatório do processo. Para o sistema do reator de polimerização escolheu-se $M=55$, sendo que a sintonia completa do SPC é apresentada na Tabela 6.5.

Tabela 6.5 - Sintonia do controlador SPC para o sistema do reator de polimerização. Valores relativos ao estado estacionário.

\begin{tabular}{lcc}
\hline \multicolumn{1}{c}{ Parâmetro de Sintonia } & Símbolo & Valor \\
\hline Matriz de pesos das saídas & $Q$ & $\operatorname{diag}\left(\left[\begin{array}{ll}10 & 5\end{array}\right]\right)$ \\
\hline Matriz de pesos das entradas & $R$ & $\operatorname{diag}\left(\left[\begin{array}{ll}0.001 & 0.001\end{array}\right]\right)$ \\
\hline Limite máximo das entradas & $u_{\max }$ & {$\left[\begin{array}{cc}50 & 60\end{array}\right]^{T}$} \\
\hline Limite mínimo das entradas & $u_{\min }$ & {$\left[\begin{array}{cc}-50 & -80\end{array}\right]^{T}$} \\
\hline Variação máxima das entradas & $\Delta u_{\max }$ & {$\left[\begin{array}{ll}2 & 2\end{array}\right]^{T}$} \\
\hline Horizonte de predição & $n p$ & 50 \\
\hline Horizonte de controle & $n c$ & 6 \\
\hline Horizonte de dados passados & $M$ & 55 \\
\hline
\end{tabular}

\subsection{SIMULAÇÃO DO SPC COM REIDENTIFICAÇÃO DO MODELO}

No intuito de analisar o desempenho do controlador SPC com excitação interna e posterior reidentificação em malha fechada para um sistema não-linear, realizou-se uma simulação com o sistema do reator de polimerização. Nessa simulação, partindo-se do estado estacionário, o processo é inicialmente controlado em $y^{s p}=\left[\begin{array}{ll}0.1 & -0.1\end{array}\right]^{T}$. No instante 100 horas, o parâmetro $A_{t}$ (fator pré-exponencial para a reação de terminação) sofre um decréscimo de $10 \%$, representando um distúrbio permanente e aumentando o erro no modelo usado no controlador. A influência dessa alteração foi analisada na seção 6.1.3. Até o instante 200 horas, a condição de excitação do SPC é mantida desativada e apenas um pequeno ruído aleatório de variância 0.00001 é adicionado à entrada do processo. No período de 200 horas a 1400 horas, a excitação interna do SPC permanece ativa. Ao final desse intervalo, realiza-se a reidentificação do modelo de predição em subespaços. Por fim, no instante 1440 horas, o controle do processo é alterado para $y^{s p}=\left[\begin{array}{ll}0 & 0.2\end{array}\right]^{T}$. 
O desempenho do SPC com reidentificação do modelo foi comparado ao desempenho do MPC com modelo constante. Considerou-se que, inicialmente, ambos os controladores utilizam um modelo linear aproximado (Modelo R1). Para tornar a comparação mais coerente, a mesma sintonia foi utilizada para ambos os controladores. Optou-se por manter a sintonia apresentada na Tabela 6.5. Os parâmetros de sintonia adicionais referentes à excitação interna do SPC e utilizados no período de 200 horas a 1400 horas estão apresentados na Tabela 6.6.

Tabela 6.6 - Sintonia adicional de excitação do controlador SPC para o sistema do reator de polimerização.

\begin{tabular}{lcc}
\hline \multicolumn{1}{c}{ Parâmetro de Sintonia } & Símbolo & Valor \\
\hline Número de direções menos excitadas & $n_{p e}$ & 10 \\
\hline Nível de excitação & $\rho$ & 450 \\
\hline Peso da excitação em relação ao controle & $P$ & 0.001 \\
\hline Intervalo de cálculo das direções & $T_{c d}$ & $30 \mathrm{~h}$ \\
\hline
\end{tabular}

A Figura 6.5 apresenta o resultado obtido na simulação:
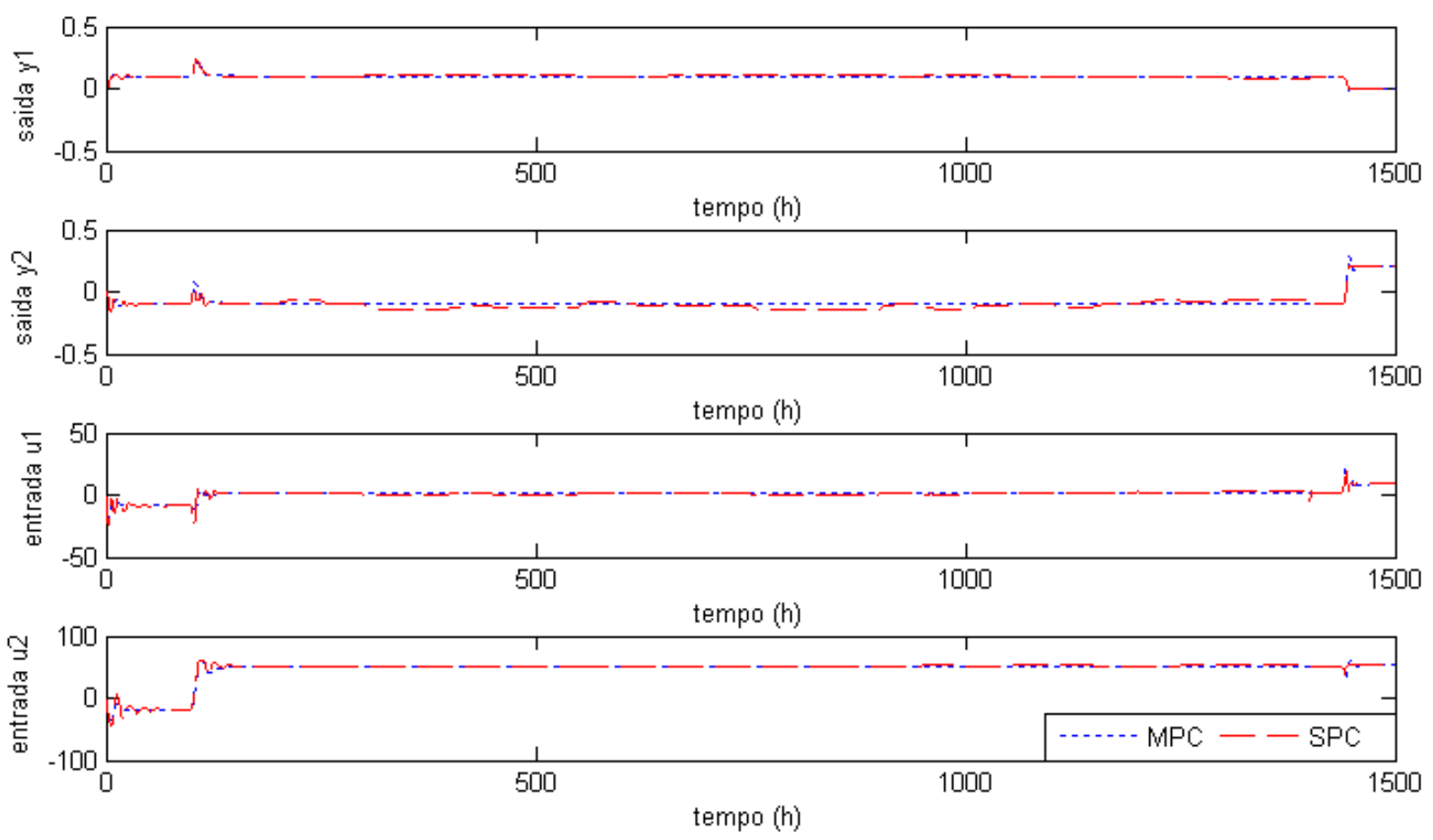

Figura 6.5 - Comparação entre MPC e SPC com reidentificação do modelo para o sistema do reator de polimerização. Visão Geral.

Para facilitar a análise dessa simulação, a Figura 6.5 foi dividida em três figuras que apresentam as variáveis do processo em intervalos de tempo menores: 

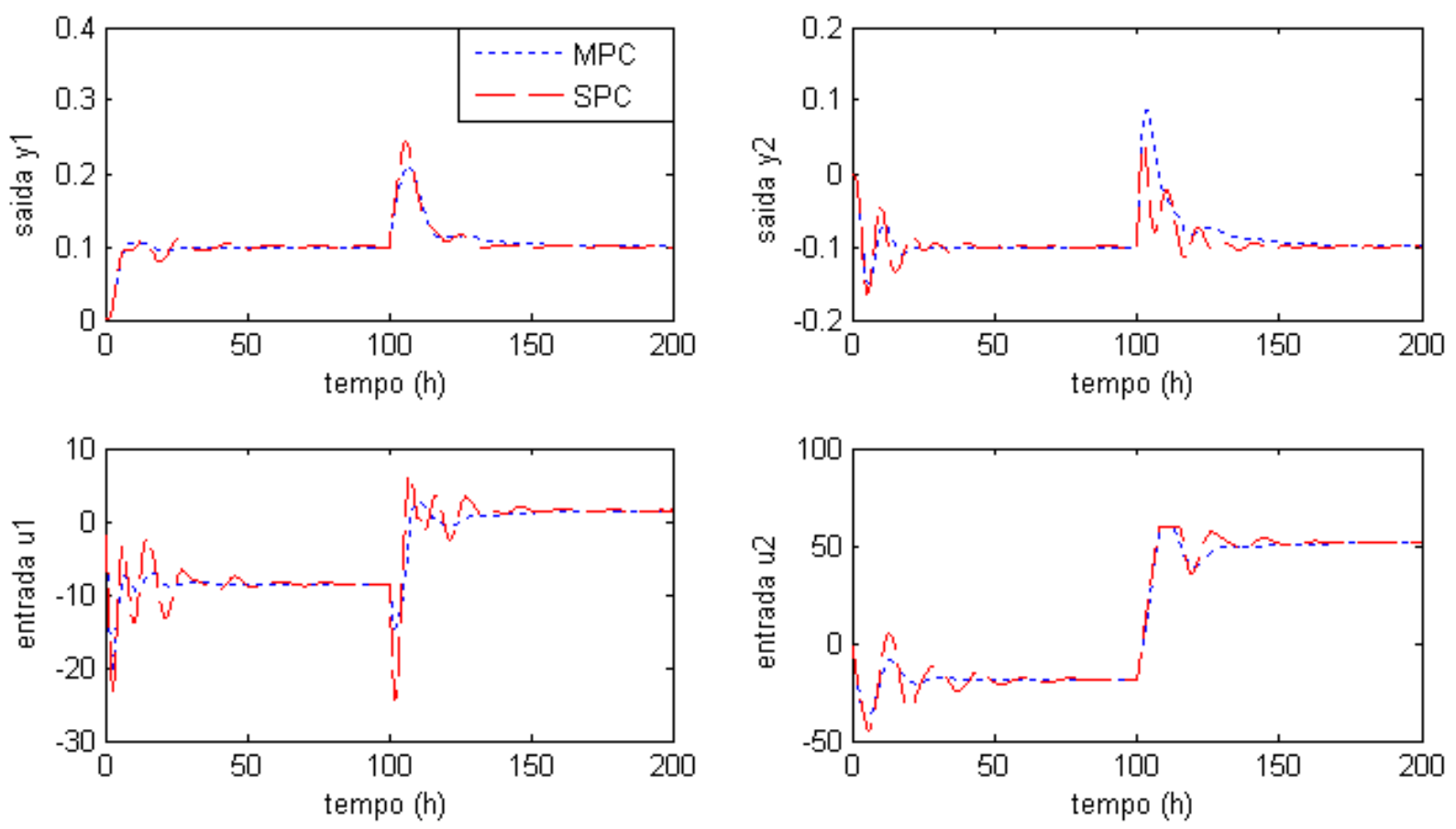

Figura 6.6 - Comparação entre MPC e SPC com reidentificação do modelo para o sistema do reator de polimerização. Ambos os controladores com modelo inicial (Período de 0 a 200 horas).
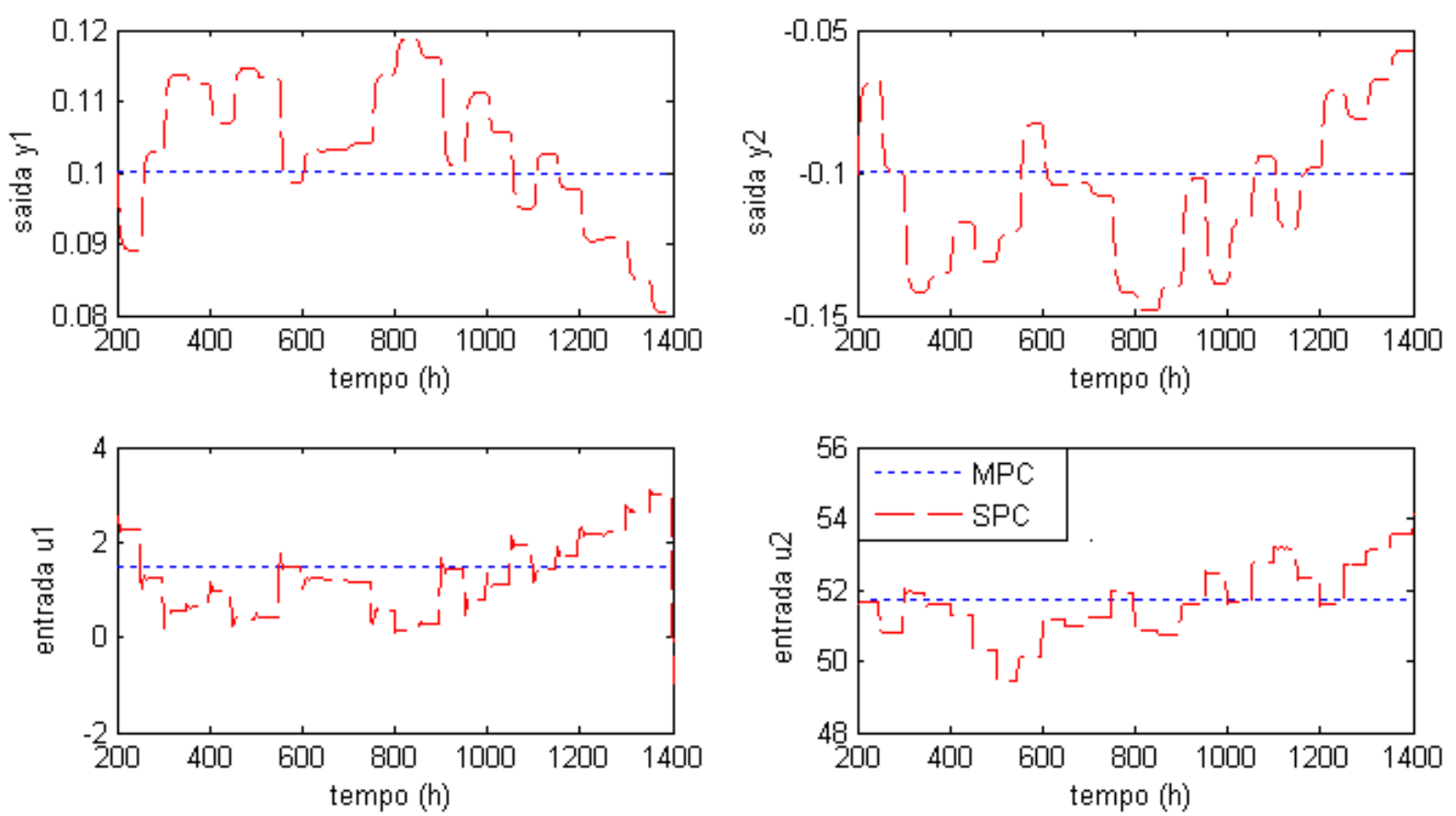

Figura 6.7 - Comparação entre MPC e SPC com reidentificação do modelo para o sistema do reator de polimerização. Controlador SPC com condição de excitação ativa (Período de 200 a 1400 horas). 

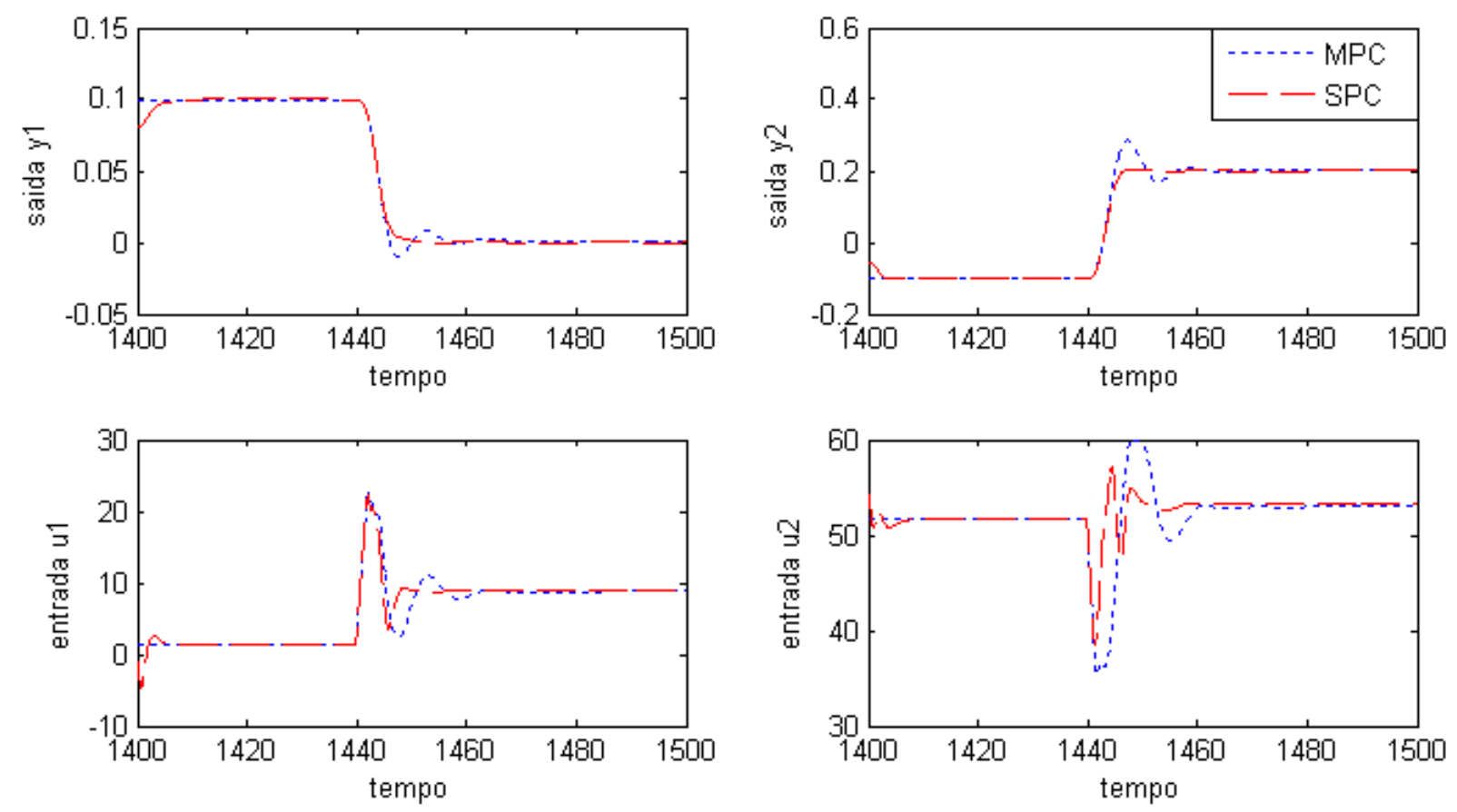

Figura 6.8 - Comparação entre MPC e SPC com reidentificação do modelo. Controlador SPC com modelo reidentificado (Período de 1400 a 1500 horas).

Analisando a Figura 6.6, nota-se que para o caso de erro nos modelos dos controladores, O MPC apresentou um desempenho superior ao SPC. Esse comportamento é coerente com o resultado obtido nas simulações com o sistema linear da coluna debutanizadora. Observa-se que, no instante 100 horas, as variáveis controladas são deslocadas de seus set-points, devido ao distúrbio gerado pela alteração no parâmetro $A_{t}$. Após certo tempo, ambos os controladores conseguem neutralizar esse distúrbio, de modo que as saídas retornam a seus valores desejados.

No intervalo de tempo de 200 horas a 1400 horas (Figura 6.7) o controlador MPC mantém as variáveis constantes, enquanto o controlador SPC inicia a excitação do sistema. Observa-se que as oscilações das variáveis controladas tendem a se manter em torno de seus set-points. Vale lembrar que esse comportamento não é garantido pelo controlador. O SPC garante apenas a permanência das variáveis manipuladas dentro de seus limites inferiores e superiores. Por fim, nota-se que as variáveis manipuladas se movimentam em degraus, permitindo a coleta de informações sobre a dinâmica lenta do sistema. 
Na Figura 6.8, observa-se que o controlador SPC com modelo reidentificado teve um desempenho superior ao controlador MPC, que permaneceu com o modelo inicial. Esse fato evidencia a eficácia da excitação imposta e do método de identificação utilizado. Além disso, o comportamento observado no caso do sistema linear é confirmado.

Durante a realização dos testes, na reidentificação do modelo de predição em subespaços, para se obter um modelo de boa qualidade, foi preciso utilizar um número grande de dados, em torno de 100 vezes o horizonte $M$. De fato, nota-se, pela Figura 6.7, que o período de excitação foi bastante longo. Isso não foi necessário nas identificações realizadas para o sistema linear da coluna debutanizadora, nas quais se utilizou apenas $10 \cdot M$ dados. Observou-se que, no caso do sistema do reator de polimerização (não-linear), identificações feitas com poucos dados amostrados resultaram no surgimento de ruídos na resposta ao degrau do sistema, como pode ser observado na Figura 6.9, que ilustra as respostas ao degrau dos modelos de predição gerados $\operatorname{com} 10 \cdot M$ e $100 \cdot M$ dados.
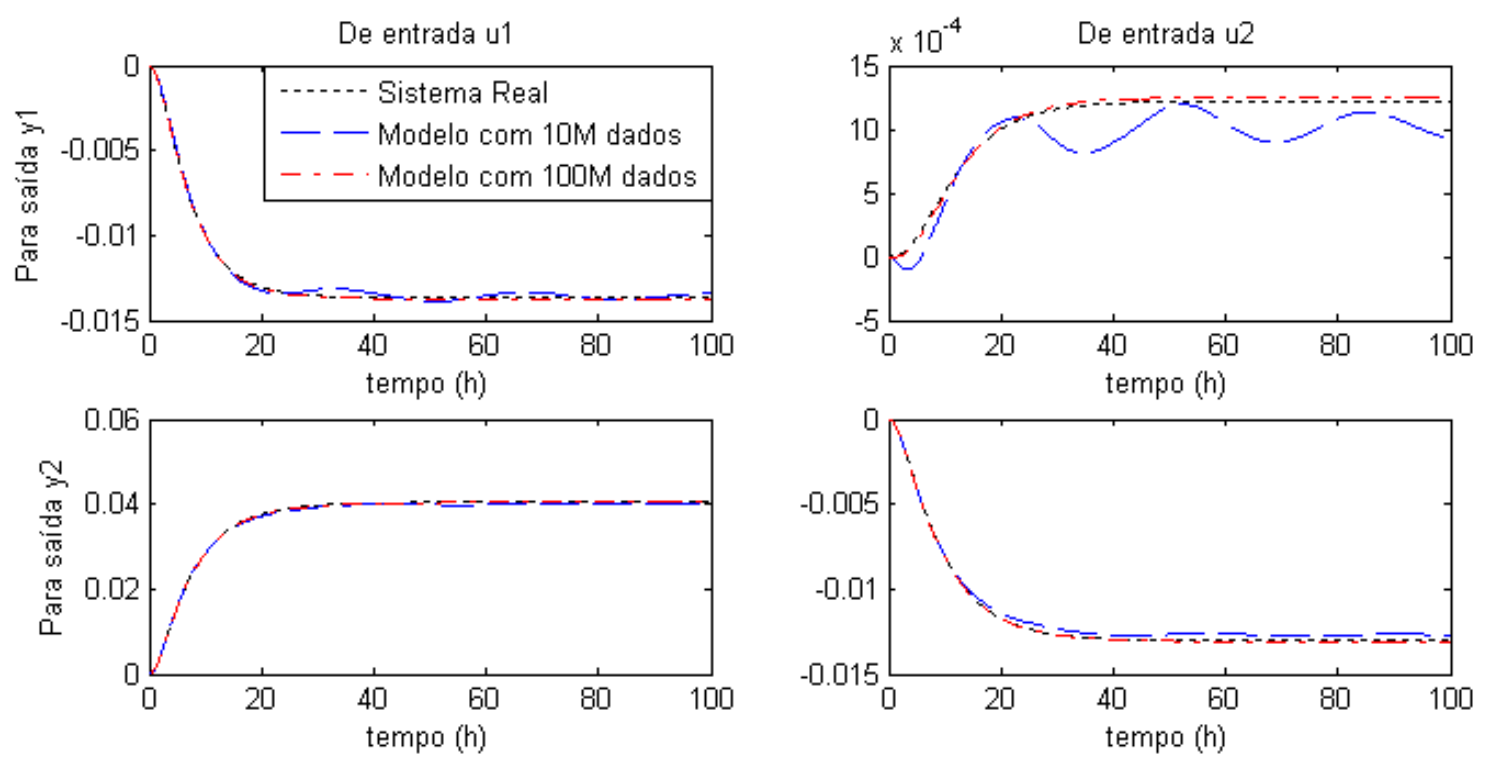

Figura 6.9 - Comparação das respostas ao degrau dos modelos de predição do sistema do reator de polimerização identificados com diferentes números de dados.

Na Figura 6.9, observa-se que o modelo gerado a partir de $10 \cdot M$ dados apresenta oscilações inexistentes no sistema real. Quando esse modelo é usado no controlador SPC, essas oscilações geram predições erradas das saídas, resultando em um controle ruim do processo. 
Uma última observação deve ser feita acerca dos testes de identificação do modelo de predição em subespaços realizados para o sistema do reator de polimerização. Para esse sistema, com dinâmica não-linear, foram utilizados dois procedimentos de identificação distintos:

- Modo 1: inicialmente aproximou-se a resposta ao degrau do sistema real por um modelo linear de primeira ordem. Esse modelo linear foi então simulado em malha aberta com excitação de um sinal do tipo PRBS. Finalmente, o modelo de predição foi obtido através de identificação a partir dos dados coletados na simulação.

- Modo 2: o sistema real foi excitado pelo controlador SPC. A partir dos dados amostrados, o modelo de predição foi identificado.

Observou-se que, dependendo da qualidade do modelo linear definido no Modo 1, esse procedimento resultava em um modelo de predição melhor do que o obtido pelo Modo 2. A princípio esse fato parece estranho, pois significa que dados coletados de um sistema aproximado são mais adequados para a identificação do que dados reais da planta. Entretanto, como o modelo de predição em subespaços assume que o sistema seja linear, o resultado observado é coerente.

$\mathrm{Na}$ identificação pelo Modo 1, a linearização do sistema é realizada em uma etapa anterior, sendo que os cálculos da identificação consistem apenas em operações algébricas para se obter o modelo no formato desejado. Porém, quando se realiza a identificação pelo Modo 2, essas transformações algébricas também assumem a função de linearizar o sistema. Em ambos os procedimentos, a qualidade do modelo final depende basicamente do resultado obtido na linearização. Se o modelo linear usado no Modo 1 for muito próximo do sistema real, esse método resultará um modelo de predição em subespaços melhor. 


\section{CONSIDERAÇÕES FINAIS}

\subsection{CONCLUSÕES}

Neste trabalho foi apresentado o método de identificação em subespaços (SID), que se baseia em conceitos da álgebra linear numérica. Ele é um método simples e robusto, não sendo necessário nenhum conhecimento prévio do sistema. Simulações realizadas para um processo típico da indústria química mostraram que - SID é uma boa alternativa para os métodos clássicos de identificação, mesmo quando os dados são coletados em malha fechada. Um problema encontrado nesse método é que quando os dados apresentam ruídos correlacionados com a entrada ou o sistema é não-linear, a determinação da ordem do modelo, feita por inspeção de valores singulares, não é trivial. Assim, em casos práticos, deve-se obter a ordem do modelo de maneira iterativa. Outra opção é utilizar algum critério para essa escolha, como, por exemplo, o Critério de Informação de Akaike.

A partir dos conceitos de SID, em conjunto com resultados da área de controle preditivo baseado em modelos (MPC), surgiu uma nova técnica de controle, denominada, neste trabalho, controle preditivo com enfoque em subespaços (SPC). Essa técnica substitui por um único passo as três etapas do projeto de um MPC: a identificação do modelo, o cálculo do observador de estados (filtro de Kalman) e a construção das matrizes de predição. A técnica SPC foi estudada por muitos autores como um método conjunto de identificação e controle. Neste trabalho, porém, demonstrou-se que é possível abordar o SPC como uma técnica de controle na qual primeiramente um modelo do processo deve ser identificado. Esse modelo, denominado modelo de predição em subespaços, é constituído das matrizes de predição do controlador, que são obtidas diretamente de dados amostrados.

No modelo de predição em subespaços, o estado atual do sistema é estimado a partir de dados passados, não sendo necessário o uso do Filtro de Kalman. Uma análise detalhada desse modelo foi realizada por meio de simulações para um 
sistema de uma coluna debutanizadora da refinaria RPBC. Mostrou-se que o horizonte de dados passados $(M)$ deve ser escolhido cuidadosamente na etapa de projeto do controlador. Ele deve ser grande o suficiente para conseguir estimar satisfatoriamente o estado do sistema. Porém, um $M$ muito elevado pode gerar grandes erros de predição, se houver ruídos no processo ou se o modelo do controlador não for perfeito. Por fim, demonstrou-se como esse modelo de predição pode ser relacionado com o modelo em espaço de estados usado no MPC.

Os controladores SPC e MPC foram aplicados para o sistema da coluna debutanizadora, considerando-se diferentes situações de controle. Para o caso ideal (processo sem ruídos e modelos dos controladores perfeitos), o MPC e o SPC se comportaram de maneira idêntica. Simulações mostraram que o SPC responde de maneira mais rápida e brusca a distúrbios adicionados à saída do processo, como erros de medição. Isso ocorre devido ao uso do Filtro de Kalman no MPC, que realiza um pré-tratamento no erro de predição antes de adicioná-lo no modelo. Esse comportamento do SPC pode ser vantajoso quando é necessário corrigir rapidamente um distúrbio indesejado, porém, na presença de pequenos ruídos de alta freqüência, ele causa muitas oscilações nas entradas do processo. Concluiu-se também que o SPC é mais dependente da qualidade do modelo que o MPC.

Foi comprovado neste trabalho que a persistência de excitação dos dados amostrados é um requisito necessário para a identificação de um bom modelo. No enfoque do SPC, foram propostos três algoritmos de excitação interna. Todos eles possuem a vantagem de manter o problema de otimização do controlador na forma quadrática. Esses algoritmos identificam direções menos excitadas pela entrada do processo e tentam forçar as ações de controle futuras nessas direções. O primeiro algoritmo necessita do cálculo do estado estacionário do sistema, que pode ser complicado ou impreciso. Os outros dois algoritmos eliminam a necessidade desse cálculo, apresentando novas alternativas de se adicionar a condição de excitação no problema de otimização.

Simulações mostraram que é possível excitar o sistema de forma satisfatória utilizando o controlador SPC com excitação interna. Além disso, esse controlador apresenta parâmetros de sintonia adicionais que permitem um ajuste fino da 
excitação desejada. Uma análise detalhada sobre a função de cada um desses parâmetros foi realizada. A aplicação do controlador SPC com excitação interna para o sistema da coluna debutanizadora demonstrou uma grande melhora no desempenho do SPC após a reidentificação do modelo com os dados excitados.

Por fim, aplicou-se o SPC com excitação interna para o sistema de um reator de polimerização com dinâmica não-linear. Observou-se, nesse caso, que o horizonte de dados passados do modelo de predição $(M)$ teve uma forte influência no desempenho do controlador. Os mesmos resultados verificados na simulação com o sistema da coluna debutanizadora foram obtidos: o desempenho do SPC apresentou grande melhora após a reidentificação do modelo com dados excitados.

Neste trabalho concluiu-se que o método SPC utiliza de forma inovadora conceitos bem consolidados das áreas de SID e MPC. Ele apresentou resultados interessantes, sendo aplicado com sucesso em dois sistemas típicos da indústria de processos químicos. Uma desvantagem dessa técnica é que para se obter um modelo de predição satisfatório, foi necessário excitar a planta por um período muito longo, principalmente para o sistema não-linear do reator de polimerização, o que nem sempre é possível em casos reais.

\subsection{SUGESTÕES DE CONTINUIDADE}

Muitos estudos têm sido feitos na área de identificação de sistemas em subespaços. Diversos métodos e algoritmos foram propostos, proporcionando grandes avanços. Porém, uma desvantagem dos métodos SID é que não é possível utilizar nenhum conhecimento prévio do sistema. Alguns trabalhos recentes foram publicados na tentativa de incorporar informações prévias do sistema no procedimento de identificação. Entretanto, essa ainda é uma área de pesquisa que deve ser explorada.

Aplicações do método SPC para o sistema da coluna debutanizadora e para o sistema do reator de polimerização apresentaram bons resultados. Porém, ambos os 
sistemas possuem dimensão dois por dois, apresentando um grau de complexibilidade relativamente pequeno. Recomenda-se testar esse controlador em sistemas MIMO que possuem mais variáveis, de modo que uma conclusão mais abrangente possa ser elaborada.

A excitação interna proposta no enfoque do SPC mostrou ser um método eficiente para a geração de dados persistentemente excitantes. Essa técnica pode ser aprimorada pela inclusão de certa aleatoriedade na escolha dos parâmetros de sintonia, característica favorável à persistência de excitação. Pode-se estudar, por exemplo, a variação aleatória dentro de faixas pré-estabelecidas do número de direções utilizadas $\left(n_{p e}\right)$ e do intervalo em que são calculadas essas direções $\left(T_{c d}\right)$.

Finalmente, o conceito de direções menos excitadas é bastante inovador e, quando utilizado no controle preditivo para garantir a excitação persistente dos dados, possui a grande vantagem de manter o problema de otimização na forma quadrática. Esse conceito pode ser estendido para o caso de identificação do modelo em espaço de estados via SID. Isso permitiria o desenvolvimento de um controlador MPC com excitação interna com as mesmas características do SPC proposto neste trabalho. 


\section{REFERÊNCIAS}

AGGELOGIANNAKI, E.; SARIMVEIS, H. Multiobjective constrained MPC with simultaneous closed-loop identification. International Journal of Adaptive Control and Signal Processing, v. 20, p. 145-173, 2006.

ALMEIDA NETO, E. Controle preditivo multimodelos de uma torre debutanizadora. 1999. 179 p. Dissertação (Mestrado) - Escola Politécnica, Universidade de São Paulo. São Paulo, 1999.

BALLIN, S. L. Controlador preditivo multivariável com restrição de excitação para identificação de processos em malha fechada. 2008. 128 p. Dissertação (Mestrado) - Escola Politécnica, Universidade de São Paulo. São Paulo, 2008.

BOMBOIS, X.; SCORLETTI, G.; GEVERS, M.; VAN DEN HOF, P. M. J.; HILDEBRAND, R. Least costly identification experiment for control. Automatica, v. 42, n. 10, p. 1651-1662, 2006.

CUTLER, C. R.; RAMAKER, B. L. Dynamic matrix control: a computer control algorithm. In: AIChE 86th National Meeting, Houston, TX, USA, 1979.

DI RUSCIO, D. (1997a) Model Predictive Control and Identification: A Linear State Space Model Approach. In: 36th Conference on Decision \& Control, San Diego, California, USA, Dec. 1997. Proceedings. p. 3202-3209.

DI RUSCIO, D. (1997b) Model Based Predictive Control: An extended state space approach. In: 36th Conference on Decision \& Control, San Diego, California, USA, Dec. 1997. Proceedings. p. 3210-3217.

DI RUSCIO, D.; FOSS, B. On State Space Model Based Predictive Control. In: 5th IFAC Symposium on Dynamics and Control of Process Systems, Corfu, Greece, June, 1998. Proceedings. p. 301-306.

FAVOREEL, W. Subspace methods for identification and control of linear and bilinear systems. 1999. 188p. Tese (Doutorado) - Katholieke Universiteit Leuven. Leuven, Belgium, 1999. 
FAVOREEL, W.; DE MOOR, B. SPC: Subspace Predictive Control. Leuven: Katholieke Universiteit Leuven, 1998. Technical report 98-49.

FAVOREEL, W.; DE MOOR, B.; VAN OVERSCHEE, P.; GEVERS, M. Model-free subspace-based LQG-design. In: American Control Conference, San Diego, California, USA, June, 1999. Proceedings. p. 3372-3376.

FAVOREEL, W.; DE MOOR, B.; VAN OVERSCHEE, P. Subspace state space system identification for industrial processes. Journal of Process Control, v. 10, n. 2-3, p. 149-155, 2000.

FORSSELL, U.; LJUNG, L. Closed-loop identification revisited. Automatica, v. 35, n. 7, p. 1215-1241, 1999.

FORSSELL, U.; LJUNG, L. Some results on optimal experiment design. Automatica, v. 36, n. 5 , p. $749-756,2000$.

GARCIA, C. Identificação de sistemas. São Paulo: EPUSP, 2008. Apostila para disciplina de pós graduação do Departamento de Engenharia Elétrica, PTC-5719 Identificação de Sistemas.

GONZÁLEZ, A. H.; ADAM, E. J.; MARCHETTI, J. L. Conditions for offset elimination in state space receding horizon controllers: A tutorial analysis. Chemical

Engineering and Processing, v. 47, n. 12, p. 2184-2194, 2008.

GUSTAVSSON, I.; LJUNG, L.; SÖDERSTRÖM, T. Identification of processes in closed loop: identifiability and accuracy aspects. Automatica, v. 13, n. 1, p. 59-75, 1977.

HALE, E. T.; QIN, S. J. Subspace Model Predictive Control and a Case Study. In: American Control Conference, Anchorage, Alaska, USA, May, 2002. Proceedings. p. $4758-4763$.

HALLOUZI, R.; VERHAEGEN, M. Persistency of excitation in subspace predictive control. In: 17th IFAC World Congress, Seoul, Korea, July, 2008. Proceedings. $p$. 11439-11444.

HIDALGO, P. M.; BROSILOW, C. B. Nonlinear model predictive control of styrene polymerization at unstable operating points. Computers \& Chemical Engineering, v. 14, n. 4/5, p. 481-494, 1990. 
HJALMARSSON, H. From experiment design to closed-loop control. Automatica, v. 41, n. 3, p. 393-438, 2005.

HJALMARSSON, H.; GEVERS, M.; DE BRUYNE, F. For Model-based Control Design, Closed-loop Identification Gives Better Performance. Automatica, v. 32, n. 12 , p. 1659-1673, 1996.

HUANG, B.; KADALI, R. Dynamic Modeling, Predictive Control and Performance Monitoring: A Data-driven Subspace Approach. London: Springer-Verlag, 2008.

KADALI, R.; HUANG, B.; ROSSITER, A. A data driven subspace approach to predictive controller design. Control Engineering Practice, v. 11, n. 3, p. 261-278, 2003.

KATAYAMA, T. Subspace Methods for System Identification. London: SpringerVerlag, 2005.

KATAYAMA, T.; TANAKA, H. An approach to closed-loop subspace identification by orthogonal decomposition. Automatica, v. 43, n. 9, p.1623-1630, 2007.

LARIMORE, W. E. Canonical Variate Analysis in Identification, Filtering, and Adaptive Control. In: 29th Conference on Decision and Control, Honolulu, Hawaii, Dec. 1990. Proceedings. p. 596-604.

LI, T.; GEORGAKIS, C. Dynamic input signal design for the identification of constrained systems. Journal of Process Control, v. 18, n. 3/4, p. 332-346, 2008.

LJUNG, L. System identification: theory for the user. $2^{\text {nd }}$ ed. New Jersey: Prentice Hall, 1999.

MACIEJOWSKI, J.M. Predictive Control with Constraints. New Jersey: Prentice Hall, 2002.

MANER, B. R.; DOYLE III, F. J.; OGUNNAIKE, B. A.; PEARSON, R. K. Nonlinear Model Predictive Control of a Simulated Multivariable Polymerization Reactor Using Second-order Volterra Models. Automatica, v. 32, n. 9, p. 1285-1301, 1996.

MICCHI, A.; PANNOCCHIA, G. Comparison of input signals in subspace identification of multivariable ill-conditioned systems. Journal of Process Control, v. 18, n. 6, p. 582-593, 2008. 
ODLOAK, D. Notas de Aula. São Paulo: EPUSP, 2008. Apostila para disciplina de pós graduação do Departamento de Engenharia Química, PQI-5780 - Controle de Processos Químicos I.

QIN, S. J.; BADGWELL, T. A. A survey of industrial model predictive control technology. Control Engineering Practice, v. 11, n. 7, p. 733-764, 2003.

QIN, S. J.; LIN, W.; LJUNG, L. A novel subspace identification approach with enforced causal models. Automatica, v. 41, n. 12, p. 2043-2053, 2005.

RAWLINGS, J. B. Tutorial Overview of Model Predictive Control. IEEE Control Systems Magazine, v. 20, n. 3, p. 38-52, 2000.

RODRIGUES, M. A.; ODLOAK, D. Output feedback MPC with guaranteed robust stability. Journal of Process Control, v. 10, n. 6, p. 557-572, 2000.

SHOUCHE, M.; GENCELI, H.; VUTHANDAM, P.; NIKOLAOU, M. Simultaneous Constrained Model Predictive Control and Identification of DARX Processes.

Automatica, v. 34, n. 12, p. 1521-1530, 1998.

SONG, I.-H.; YOO, K.-Y.; RHEE, H.-K. Analysis and Design of a Linear Input/Output Data-Based Predictive Control. Industrial Engineering Chemistry Research, v. 40, p. 4292-4301, 2001.

SOTOMAYOR, O. A. Z.; ODLOAK, D.; GIUDICI, R. Diagnosis of Abnormal Situations in a Continuous Solution Polymerization Reactor. Macromolecular Theory and Simulations, v. 16, p. 247-261, 2007.

SOTOMAYOR, O. A. Z.; ODLOAK, D.; MORO, L. F. L. Closed-loop model reidentification of processes under MPC with zone control. Control Engineering Practice, v. 17, n. 5, p. 551-563, 2009.

VAN OVERSCHEE, P.; DE MOOR, B. N4SID: Subspace Algorithms for the Identification of Combined Deterministic-Stochastic Systems, Automatica, v. 30, n. 1, p. 75-93, 1994.

VAN OVERSCHEE, P.; DE MOOR, B. A Unifying Theorem for Three Subspace System Identification Algorithms. Automatica, v. 31, n. 12, p. 1853-1864, 1995. 
VAN OVERSCHEE, P.; DE MOOR, B. SUBSPACE IDENTIFICATION FOR LINEAR SYSTEMS: Theory - Implementation - Applications. Kluwer Academic Publishers, 1996.

VAN OVERSCHEE, P.; DE MOOR, B. Closed Loop Subspace System Identification. In: 36th Conference on Decision \& Control, San Diego, California, USA, Dec. 1997. Proceedings. p. 1848-1853.

VERHAEGEN, M. Identification of the Deterministic Part of MIMO State Space Models given in Innovations Form from Input-Output Data. Automatica, v. 30, n. 1, p. 61-74, 1994.

WANG, X.; HUANG, B.; CHEN, T. Data-driven predictive control for solid oxide fuel cells. Journal of Process Control, v. 17, n. 2, p. 103-114, 2007.

WANG, J.; QIN, S. J. Closed-loop subspace identification using the parity space. Automatica, v. 42, n. 2, p. 315-320, 2006.

WANG, L.; YOUNG, P. C. An improved structure for model predictive control using non-minimal state space realisation. Journal of Process Control, v. 16, n. 4, p. 355371, 2006.

ZHU, Y. Multivariable System Identification: for Process Control. $1^{\text {st }}$ ed. Pergamon Press, 2001. 